NI-94-017

CTP/TAMU-67/92

McGill/94-53

CERN-TH.7542/94

hep-th/9412184

\title{
String Solitons
}

\author{
M. J. Duff ${ }^{1,2 \dagger}$, Ramzi R. Khuri ${ }^{3,4 *}$ and J. X. $\mathrm{Lu}^{2 * *}$ \\ ${ }^{1}$ Isaac Newton Institute, Cambridge University \\ 20 Clarkson Road, Cambridge CB3 OEH U.K. \\ ${ }^{2}$ Center for Theoretical Physics, Texas A $\mathscr{G} M$ University \\ College Station, TX 77843 USA \\ ${ }^{3}$ Physics Department, McGill University \\ 3600 University, St. Montréal, PQ, H3A 2T8 Canada \\ ${ }^{4}$ Theory Division, CERN \\ CH-1211, Geneva 23, Switzerland
}

$12 / 94$

$\dagger$ Work supported in part by NSF grant PHY-9411543.

* Work supported in part by a World Laboratory Fellowship.

** Current address: Department of Nuclear Engineering, Texas A\&M University, College Station, TX 77843 USA 


\begin{abstract}
We review the status of solitons in superstring theory, with a view to understanding the strong coupling regime. These solitonic solutions are non-singular field configurations which solve the empty-space low-energy field equations (generalized, whenever possible, to all orders in $\alpha^{\prime}$ ), carry a non-vanishing topological "magnetic" charge and are stabilized by a topological conservation law. They are compared and contrasted with the elementary solutions which are singular solutions of the field equations with a $\sigma$-model source term and carry a non-vanishing Noether "electric" charge. In both cases, the solutions of most interest are those which preserve half the spacetime supersymmetries and saturate a Bogomol'nyi bound. They typically arise as the extreme mass=charge limit of more general two-parameter solutions with event horizons. We also describe the theory dual to the fundamental string for which the roles of elementary and soliton solutions are interchanged. In ten spacetime dimensions, this dual theory is a superfivebrane and this gives rise to a string/fivebrane duality conjecture according to which the fivebrane may be regarded as fundamental in its own right, with the strongly coupled string corresponding to the weakly coupled fivebrane and vice-versa. After compactification to four spacetime dimensions, the fivebrane appears as a magnetic monopole or a dual string according as it wraps around five or four of the compactified dimensions. This gives rise to a four-dimensional string/string duality conjecture which subsumes a Montonen-Olive type duality in that the magnetic monopoles of the fundamental string correspond to the electric winding states of the dual string. This leads to a duality of dualities whereby under string/string duality the strong/weak coupling $S$-duality trades places with the minimum/maximum length $T$ duality. Since these magnetic monopoles are extreme black holes, a prediction of $S$-duality is that the corresponding electric massive states of the fundamental string are also extreme black holes. This is indeed the case.
\end{abstract}




\section{Table of Contents}

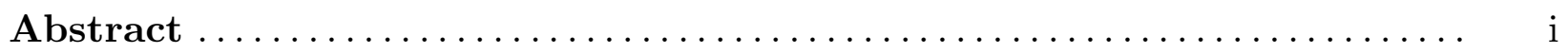

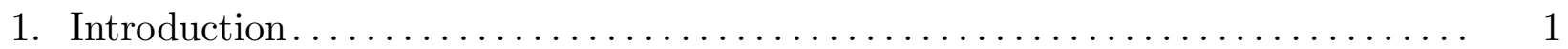

1.1. Preliminaries....................................... 1

1.2. Supersymmetric extended objects as solitons: the brane-scan . . . . . . . . 8

1.3. The Montonen-Olive conjecture $\ldots \ldots \ldots \ldots \ldots \ldots \ldots \ldots \ldots \ldots \ldots \ldots \ldots \ldots \ldots$

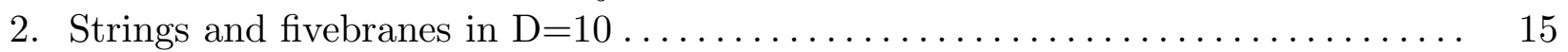

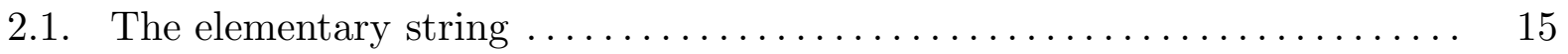

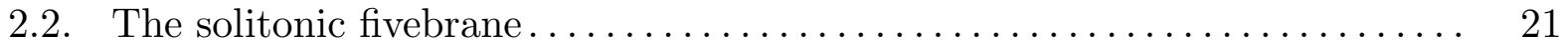

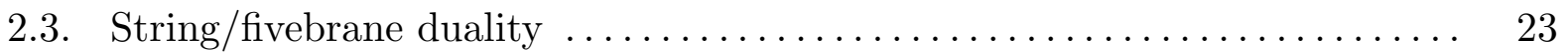

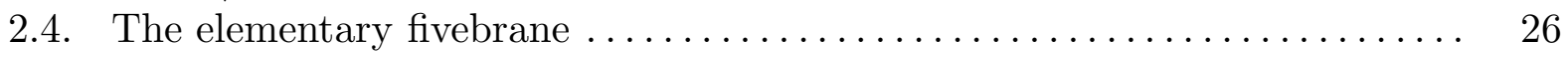

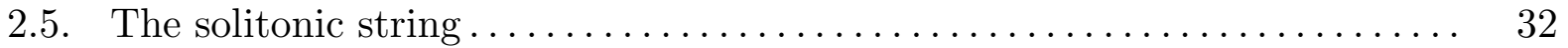

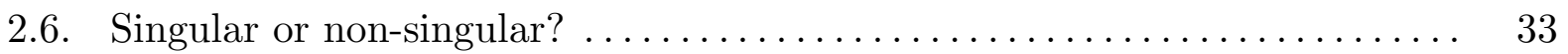

2.7. Strings and fivebranes as interpolating solitons $\ldots \ldots \ldots \ldots \ldots \ldots \ldots \ldots . \ldots \ldots$

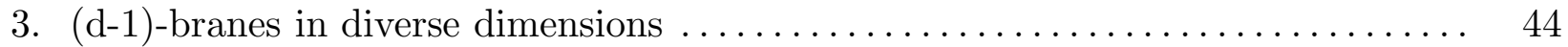

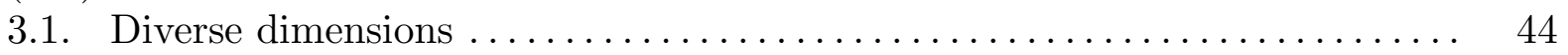

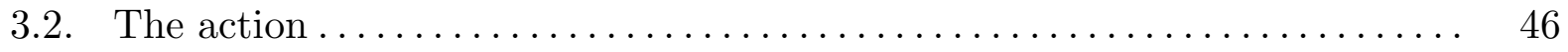

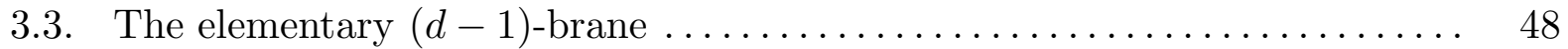

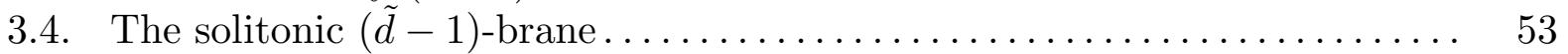

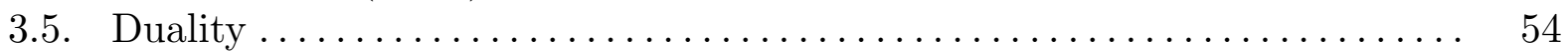

3.6. The self-dual string and dyonic string in $D=6 \ldots \ldots \ldots \ldots \ldots \ldots \ldots . \ldots 9$

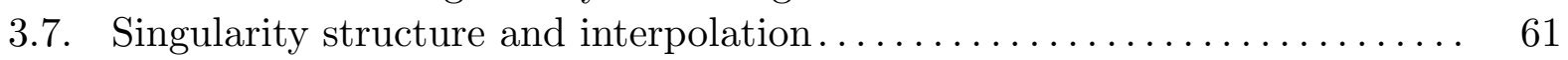

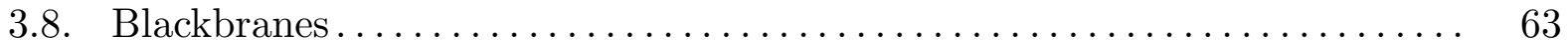

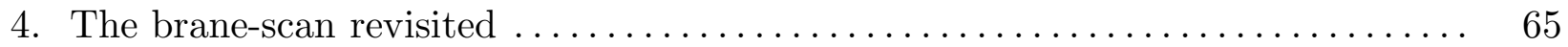

4.1. Bose-Fermi matching: a necessary condition ................... 65

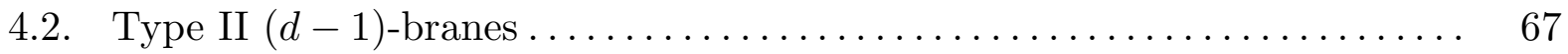

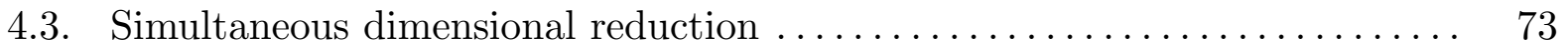

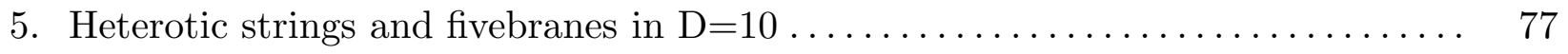

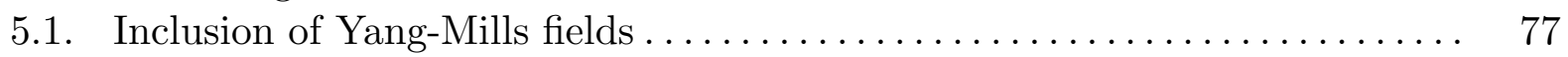

5.2. 't Hooft ansatz and the axionic instanton ..................... 78

5.3. The heterotic fivebrane as a soliton: "gauge" versus "symmetric" ....... 80

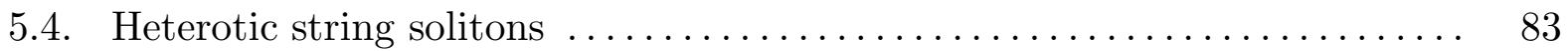

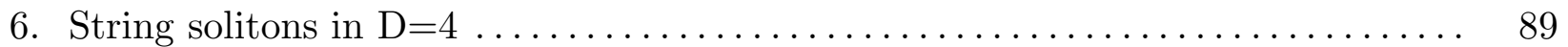

6.1. String compactification to four dimensions $\ldots \ldots \ldots \ldots \ldots \ldots \ldots \ldots \ldots \ldots$

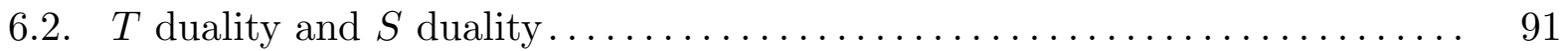

6.3. Fivebrane compactification to four dimensions.................. 92

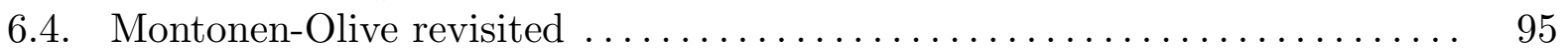

6.5. Monopoles, strings and domain walls $\ldots \ldots \ldots \ldots \ldots \ldots \ldots \ldots \ldots \ldots . \ldots \ldots$

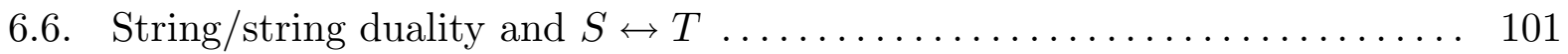

6.7. Kaluza-Klein black holes in string theory $\ldots \ldots \ldots \ldots \ldots \ldots \ldots \ldots \ldots \ldots$

6.8. Massive string states as extreme black holes..................... 112

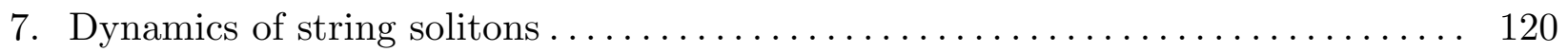

7.1. Metric on moduli space ................................ 120 
7.2. Veneziano amplitude for elementary strings $\ldots \ldots \ldots \ldots \ldots \ldots \ldots \ldots \ldots \ldots$

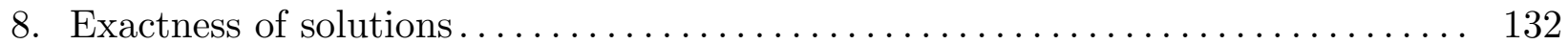

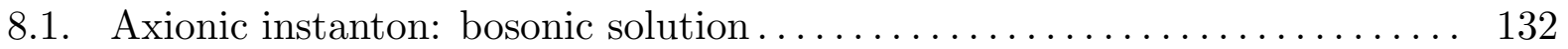

8.2. Symmetric fivebrane ............................. 136

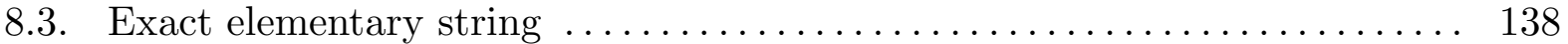

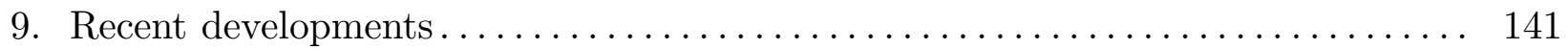

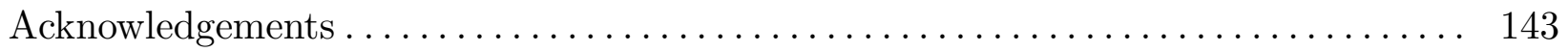

References.................................................... 144 


\section{Introduction}

\subsection{Preliminaries}

Solitons [1,2,3], sometimes called topological defects [4], are important in quantum field theory for a variety of reasons. Their existence means that the full non-perturbative theory may have a much richer structure than is apparent in perturbation theory. For example, the electrically charged elementary particle spectrum may need to be augmented by magnetically charged solitonic particles. The former carry a Noether charge following from the equations of motion while the latter carry a topological charge associated with the Bianchi identities. That these magnetic monopoles are intrinsically non-perturbative is apparent from their mass formula which depends inversely on the coupling constant. The spectrum might also contain dyons, particles carrying both electric and magnetic charge. In four spacetime dimensions, extended solitonic objects, strings and domain walls, are also possible. In modern parlance, they might be known as $(d-1)$-branes with $d=1,2,3$ where $d$ is the dimension of the worldvolume swept out by the soliton. In this report we shall use the word solitons to mean any such non-singular lumps of field energy which solve the field equations, which have finite mass per unit $(d-1)$-volume and which are prevented from dissipating by some topological conservation law. Their existence, which in some grand unified theories is actually mandatory, has far-reaching implications both at the microscopic and cosmic scales [5].

However, it is now commonly believed that ordinary quantum field theory is inadequate for a unification of all the forces including gravity and that it must be supplanted by

superstring theory. If this is true then soliton solutions of string theory must be even more important. Although the revival of the string idea is now ten years old, it is only recently that much attention has been devoted to the subject of solitons. This interest has in part been brought about by the realization that the really crucial questions of string theory: "How does the string choose a vacuum state?"; "How does the string break supersymmetry?"; "How does the string cope with the cosmological constant problem?" cannot be answered within the framework of a weak coupling perturbation expansion. Consequently, despite the enormous number of reviews on superstring theory, comparatively little has 
been written on bringing together this new wealth of information on its non-perturbative sector and trying to make sense of it. The purpose of this Report is to do just that.

The current interest in string solitons has another, ostensibly different, origin. While all the activity in superstring theory was going on a small but dedicated group of theorists was asking a seemingly very different question: if we are going to replace 0-dimensional point particles by 1-dimensional strings, why not 2-dimensional membranes or in general $(d-1)$-dimensional objects or "( $d-1)$-branes"? Since superstrings can have $D \leq 10$ spacetime dimensions, there is plenty of room for higher dimensional extended objects with $d<D$. Progress in super $(d-1)$-branes was hampered by the belief that $\kappa$-symmetry, so crucial to Green-Schwarz superparticles $(d=1)$ [6] and superstrings $(d=2)$ [7], could not be generalized to $d>2$. The breakthrough came when Hughes et al. [8] showed that $\kappa$ symmetry could, in fact, be generalized and proceeded to construct a threebrane displaying an explicit $D=6, N=1$ spacetime supersymmetry and $\kappa$-invariance on the worldvolume. Moreover, the motivation for this paper was precisely to find the superthreebrane as a topological defect of a supersymmetric field theory in $D=6$. The discovery of the other supermembranes proceeded in the opposite direction. First of all, Bergshoeff et al. [9] found corresponding Green-Schwarz actions for other values of $d$ and $D$, in particular, the eleven-dimensional supermembrane. We shall discuss this theory in section 4 and show how to derive from it the Type IIA string in ten dimensions by a simultaneous dimensional reduction of the worldvolume and the spacetime. Their method was to show such Green-Schwarz super $p$-brane actions are possible whenever there is a closed $(p+2)$ form in superspace. As described in section 1.2, the four "fundamental" super $p$-branes are then given by $p=2$ in $D=11, p=5$ in $D=10, p=3$ in $D=6$ and $p=2$ in $D=4$ [10]. Applying the above mentioned simultaneous reduction $k$ times, we find four sequences of $(p-k)$-branes in $(D-k)$ dimensions, which include the well known GreenSchwarz superstrings in $D=10,6,4$ and 3. These four sequences, known as the octonionic $(\mathcal{O})$, quaternionic $(\mathcal{H})$, complex $(\mathcal{C})$ and real $(\mathcal{R})$ sequences, make up the brane-scan of section 1.2 .

Of particular interest was the $D=10$ fivebrane, whose Wess-Zumino term coupled to a rank six antisymmetric tensor potential $A_{M N P Q R S}$ just as the Wess-Zumino term of the string coupled to a rank two potential $B_{M N}$. Spacetime supersymmetry therefore demanded that the fivebrane coupled to the 7 -form field strength formulation of $D=10$ supergravity [11] just as the string coupled to the 3 -form version $[12,13]$. These dual formulations of $D=10$ supergravity have long been something of an enigma from the 
point of view of superstrings. As field theories, each seems equally valid. In particular, provided we couple them to $E_{8} \times E_{8}$ or $S O(32)$ super-Yang-Mills, then both are anomaly free $[14,15,16]$. Since the 3 -form version corresponds to the field theory limit of the heterotic string, Duff conjectured [17] that there ought to exist a heterotic fivebrane which could be viewed as a fundamental anomaly-free theory in its own right and whose field theory limit corresponds to the dual 7-form version. We shall refer to this as the string/fivebrane duality conjecture. One of the purposes of this Report will be to summarize the evidence in its favor. At this stage, however, the solitonic element had not yet been introduced.

The next development came when Townsend [18] pointed out that not merely the $D=6$ threebrane but all the points on the $\mathcal{H}, \mathcal{C}, \mathcal{R}$ sequences correspond to topological defects of some globally supersymmetric field theory which break half the spacetime supersymmetries. This partial breaking of supersymmetry, already discussed in [8], is a key idea and is intimately connected with the worldvolume $\kappa$-symmetry which allows one to gauge away half of the fermionic degrees of freedom. He conjectured that the $p$-branes in the $\mathcal{O}$ sequence would also admit such a solitonic interpretation within the context of supergravity. Another purpose of this Report will be to examine to what extent this conjecture is true.

The first hint in this direction came from Dabholkar et al. [19], who presented a multistring solution which in $D=10$ indeed breaks half the supersymmetries. They obtained the solution by solving the low-energy 3-form supergravity equations of motion coupled to a string $\sigma$-model source and demonstrated that it saturated a Bogomol'nyi bound and satisfied an associated zero-force condition, these properties being intimately connected with the existence of unbroken spacetime supersymmetry. The authors of [19] went on to interpret these solutions as macroscopic fundamental string states and presented evidence in favour of this conjecture. Since that time, further evidence has been obtained (e.g. $[20,21,22])$ to support this identification. In section 2.1 we rederive this string solution and point out the existence of the Bogomol'nyi bound between the ADM mass per unit length and charge per unit length and discuss the zero-force condition which arises from the preservation of half the spacetime supersymmetries.

However, this $D=10$ string was clearly not the soliton anticipated by Townsend because it described a singular configuration with a $\delta$-function source at the string location. Moreover, its charge per unit length $e_{2}$ was an "electric" Noether charge associated with the equation of motion of the antisymmetric tensor field rather than a "magnetic" topological charge associated with the Bianchi identities. Consequently, in the current literature on 
the subject, this solution is now referred to as the "fundamental" or "elementary" string. Similarly, the supermembrane solution of $D=11$ supergravity found in [23] did not seem to be solitonic either because it was also obtained by coupling to a membrane $\sigma$-model source. ${ }^{\dagger}$

The next major breakthrough for $p$-branes as solitons came with the paper of Strominger [24], who showed that $D=10$ supergravity coupled to super Yang-Mills (without a $\sigma$-model source), which is the field theory limit of the heterotic string [25], admits as a solution the heterotic fivebrane. In contrast to the elementary string, this fivebrane is a genuine soliton, being everywhere nonsingular and carrying a topological magnetic charge $g_{6}$. A crucial part of the construction was a Yang-Mills instanton in the four directions transverse to the fivebrane. He went on to suggest a complete strong/weak coupling duality with the strongly coupled string corresponding to the weakly coupled fivebrane and viceversa, thus providing a solitonic interpretation of the string/fivebrane duality conjecture. In this form, string/fivebrane duality is in a certain sense an analog of the Montonen-Olive conjecture discussed in section 1.3, according to which the magnetic monopole states of four-dimensional spontaeously broken supersymmetric Yang-Mills theories may be viewed from a dual perspective as fundamental in their own right and in which the roles of the elementary and solitonic states are interchanged.

This strong/weak coupling was subsequently confirmed from the point of view of Poincaré duality in [26]. There it was shown that the just as the string loop expansion parameter is given by $\mathrm{g}_{2}=e^{\phi_{0}}$, where $\phi_{0}$ is the dilaton vev, so the analogous fivebrane parameter is given by $\mathrm{g}_{6}=e^{-\phi_{0} / 3}$ and hence that

$$
\mathrm{g}_{6}=\mathrm{g}_{2}^{-1 / 3}
$$

The same paper also established a Dirac quantization rule

$$
\kappa^{2} T_{2} T_{6}=n \pi, \quad n=\text { integer }
$$

relating the fivebrane tension $T_{6}$ to the string tension $T_{2}$, which followed from the corresponding rule for the electric and magnetic charges generalized to extended objects $[27,28]$ $e_{2} g_{6}=2 n \pi$.

$\dagger$ Curiously, however, as discussed in section 3.7, the curvature computed from its $\sigma$ model metric is finite at the location of the source, in contrast to the case of the elementary string. 
For the purposes of generalizing the Dirac quantization rule for extended objects, it is instructive to write the Maxwell's equations in terms of the electromagnetic tensor $F_{M N}$ as

$$
\begin{gathered}
\partial_{[M} F_{N P]}=0, \\
\partial_{[M}{ }^{*} F_{N P]}={ }^{*} J_{M N P},
\end{gathered}
$$

where

$$
{ }^{*} F^{M N}=\frac{1}{2} \varepsilon^{M N O P} F_{O P}, \quad{ }^{*} J^{M N O}=\varepsilon^{M N O P} J_{P},
$$

and

$$
J^{M}=(\rho, \mathbf{j}), \quad F^{0 m}=E^{m}, \quad F^{m n}=\epsilon^{m n l} B_{l},
$$

and in the absence of the monopole, the field strength $F_{M N}$ can be written in terms of the four-vector potential $A^{M}=(\phi, \mathbf{A})$ as

$$
F_{M N} \equiv 2 \partial_{[M} A_{N]}=\partial_{M} A_{N}-\partial_{N} A_{M} .
$$

The asymmetry between the equation for $F$ and that for ${ }^{*} F$ corresponds physically to the statement that there are no magnetic monopoles. If we want to restore the symmetry of the source-free Maxwell's equations by introducing magnetic monopoles then we must replace (1.7) by

$$
F_{M N}=\partial_{M} A_{N}-\partial_{N} A_{M}+\omega_{M N},
$$

so that

$$
\partial_{[M} F_{N P]}=X_{M N P},
$$

with

$$
X_{M N P}=\partial_{[M} \omega_{N P]},
$$

and hence

$$
\partial_{[M} X_{N P Q]} \equiv 0 .
$$

The monopole may be of the Dirac type where $X$ is singular

$$
X_{123}=g \delta^{3}(y),
$$

in analogy with the elementary electric charge

$$
{ }^{*} J_{123}=e \delta^{3}(y),
$$


or the source may be smeared out so as to be regular at the origin as in the 't HooftPolyakov monopole. In both cases, we have

$$
\begin{gathered}
e \equiv \int_{S^{2}} * F=\int_{M^{3}} * J, \\
g \equiv \int_{S^{2}} F=\int_{M^{3}} X .
\end{gathered}
$$

In the language of (1.4), (1.8) and (1.9), the electric charge is conserved by virtue of the field equations and hence corresponds to a Noether charge, whereas the magnetic charge is identically conserved and corresponds to a topological charge.

Just as a charged particle couples to an Abelian vector potential $A_{M}$ displays a gauge invariance

$$
A_{M} \rightarrow A_{M}+\partial_{M} \Lambda
$$

and has a gauge invariant field strength

$$
F_{M N}=2 \partial_{[M} A_{N]} \equiv \partial_{M} A_{N}-\partial_{N} A_{M}
$$

a string couples to a rank-2 antisymmetric tensor potential $A_{M N}=-A_{N M}$ with a gauge invariance

$$
A_{M N} \rightarrow A_{M N}+\partial_{[M} \Lambda_{N]}
$$

and field strength

$$
F_{M N P}=3 \partial_{[M} A_{N P]}
$$

In general, a $(d-1)$-brane couples to a $d$-form $A_{M_{1} M_{2} \cdots M_{d}}$ with

$$
A_{M_{1} M_{2} \cdots M_{d}} \rightarrow A_{M_{1} M_{2} \cdots M_{d}}+\partial_{\left[M_{1}\right.} \Lambda_{\left.M_{2} \cdots M_{d}\right]}
$$

and

$$
F_{M_{1} M_{2} \cdots M_{d+1}}=(d+1) \partial_{\left[M_{1}\right.} A_{\left.M_{2} \cdots M_{d+1}\right]} .
$$

In the language of differential forms we may write for arbitrary $d$ and $D$

$$
A_{d} \rightarrow A_{d}+d \Lambda_{d-1}
$$

and

$$
F_{d+1}=d A_{d}
$$


from which the Bianchi identity

$$
d F_{d+1} \equiv 0
$$

follows immediately. In the absence of other interactions, the equation of motion for the $d$-form potential is

$$
d^{*} F_{D-d-1}={ }^{*} J_{D-d},
$$

where the source $J$ is a $d$-form. Here we have introduced the Hodge dual operation * which converts a $d$-form into a $(D-d)$-form, e.g.

$$
\left({ }^{*} J\right)^{M_{1} M_{2} \cdots M_{D-d}} \equiv \frac{1}{d !} \varepsilon^{M_{1} M_{2} \cdots M_{D}} J_{M_{D-d+1} \cdots M_{D}},
$$

where $\varepsilon^{M_{1} \cdots M_{D}}$ is the $D$-dimensional alternating symbol with $\varepsilon^{01 \cdots D-1}=1$.

Just as the usual Maxwell's equations, (1.23), (1.24) and (1.25) imply the presence of an "electric" charge, i.e. a $(d-1)$-brane, but no "magnetic" charge, i.e. no $(D-d-3)$ brane. To restore the duality symmetry by introducing a $(D-d-3)$-brane we must modify (1.23) to

$$
F_{d+1}=d A_{d}+\omega_{d+1},
$$

so that the Bianchi identity (1.24) becomes

$$
d F_{d+1}=X_{d+2},
$$

with

$$
X_{d+2}=d \omega_{d+1} .
$$

Once again $X$ may be singular

$$
X_{123 \cdots d+2}=g_{D-d-2} \delta^{d+2}(y),
$$

or may be smeared out so as to be regular at the origin. We then have

$$
\begin{gathered}
e_{d}=\int_{S^{D-d-1}}{ }^{*} F_{D-d-1}=\int_{M^{D-d}}{ }^{*} J_{D-d}, \\
g_{D-d-2}=\int_{S^{d+1}} F_{d+1}=\int_{M^{d+2}} X_{d+2} .
\end{gathered}
$$


The Dirac quantization condition is again obtained by using the generalization of either the Dirac string [28] or Wu-Yang construction [27] as

$$
\frac{e_{d} g_{D-d-2}}{4 \pi}=\frac{1}{2}(n=\text { integer })
$$

Note that, $e_{d}$ and $g_{D-d-2}$ are not in general dimensionless but rather

$$
\left[e_{d}\right]=-\frac{1}{2}(D-2 d-2), \quad\left[g_{D-2 d-2}\right]=\frac{1}{2}(D-2 d-2)
$$

They do become dimensionless when

$$
D=2(d+1)
$$

of which the point particle $(d=1)$ in $D=4$ is the most familiar special case.

In keeping with the viewpoint that the fivebrane may be regarded as fundamental in its own right, Duff and $\mathrm{Lu}[29]$ then constructed the elementary fivebrane solution by coupling the 7 -form version of supergravity to a fivebrane $\sigma$-model source in analogy with the elementary string. This carries an electric charge $\tilde{e}_{6}$. String/fivebrane duality then suggested that by coupling the 7-form version of supergravity to super Yang-Mills (without a $\sigma$-model source), one ought to find a nonsingular heterotic string soliton carrying a topological magnetic charge $\tilde{g}_{2}$. Here one would expect an eight-dimensional Yang-Mills instanton in the eight directions transverse to the string. This was indeed the case [30], but scaling arguments required an unconventional Yang-Mills Lagrangian, quartic in the field strengths, which, however, is only to be expected in a fivebrane loop expansion [30,31].

Somewhat surprisingly, the elementary fivebrane, as pointed out by Callan, Harvey and Strominger [32,33], could also be regarded as a soliton when viewed from the dual perspective, with $g_{6}=\tilde{e}_{6}$. In other words, it provides a nonsingular solution of the source-free 3-form equations even without the presence of Yang-Mills fields. By the same token, when viewed from the dual perspective, the elementary string provides a nonsingular solution of the source-free 7 -form equations with $\tilde{g}_{2}=e_{2}$ [34].

\subsection{Supersymmetric extended objects as solitons: the brane-scan}

As the $p$-brane moves through spacetime, its trajectory is described by the functions $X^{M}(\xi)$ where $X^{M}$ are the spacetime coordinates $(M=0,1, \ldots, D-1)$ and $\xi^{i}$ are the 
worldvolume coordinates $(i=0,1, \ldots, d-1)$. It is often convenient to make the so-called "static gauge choice" by making the $D=d+(D-d)$ split

$$
X^{M}(\xi)=\left(X^{\mu}(\xi), Y^{m}(\xi)\right)
$$

where $\mu=0,1, \ldots, d-1$ and $m=d, \ldots, D-1$, and then setting

$$
X^{\mu}(\xi)=\xi^{\mu}
$$

Thus the only physical worldvolume degrees of freedom are given by the $(D-d) Y^{m}(\xi)$. So the number of on-shell bosonic degrees of freedom is

$$
N_{B}=D-d
$$

To describe the super $p$-brane we augment the $D$ bosonic coordinates $X^{M}(\xi)$ with anticommuting fermionic coordinates $\theta^{\alpha}(\xi)$. Depending on $D$, this spinor could be Dirac, Weyl, Majorana or Majorana-Weyl. The fermionic $\kappa$-symmetry means that half of the spinor degrees of freedom are redundant and may be eliminated by a physical gauge choice. The net result is that the theory exhibits a d-dimensional worldvolume supersymmetry where the number of fermionic generators is exactly half of the generators in the original spacetime supersymmetry. This partial breaking of supersymmetry is a key idea. Let $M$ be the number of real components of the minimal spinor and $N$ the number of supersymmetries in $D$ spacetime dimensions and let $m$ and $n$ be the corresponding quantities in $d$ worldvolume dimensions. Let us first consider $d>2$. Since $\kappa$-symmetry always halves the number of fermionic degrees of freedom and going on-shell halves it again, the number of on-shell fermionic degrees of freedom is

$$
N_{F}=\frac{1}{2} m n=\frac{1}{4} M N
$$

Worldvolume supersymmetry demands $N_{B}=N_{F}$ and hence

$$
D-d=\frac{1}{2} m n=\frac{1}{4} M N .
$$

A list of dimensions, number of real components of the minimal spinor and possible supersymmetries is given in Table 1 , from which we see that there are only 8 solutions to (1.40) all with $N=1$, as shown in Fig. 1 . We note in particular that $D_{\max }=11$ since $M \geq 64$ for $D \geq 12$ and hence (1.40) cannot be satisfied. Similarly $d_{\max }=6$ since $m \geq 16$ for 
$d \geq 7$. The case $d=2$ is special because of the ability to treat left and right moving modes independently. If we require the sum of both left and right moving bosons and fermions to be equal, then we again find the condition (1.40). This provides a further 4 solutions all with $N=2$, corresponding to Type II superstrings in $D=3,4,6$ and 10 (or 8 solutions in all if we treat Type IIA and Type IIB separately. The gauge-fixed Type IIB superstring will display $(8,8)$ supersymmetry on the worldsheet and the Type IIA will display $(16,0)$, the opposite $[32,33]$ of what one might naively expect). If we require only left (or right) matching, then (1.40) is replaced by

$$
D-2=n=\frac{1}{2} M N,
$$

which allows another 4 solutions in $D=3,4,6$ and 10 , all with $N=1$. The gauge-fixed theory will display $(8,0)$ worldsheet supersymmetry. The heterotic string falls into this category. The results are shown in Fig. 1 [10].

An equivalent way to arrive at the above conclusions is to list all scalar supermultiplets in $d \geq 2$ dimensions and to interpret the dimension of the target space, $D$, by

$$
D-d=\text { number of scalars. }
$$

A useful reference is [35], which provides an exhaustive classification of all unitary representations of supersymmetry with maximum spin 2 . In particular, we can understand $d_{\max }=6$ from this point of view since this is the upper limit for scalar supermultiplets. In summary, according to the above classification, Type II $p$-branes do not exist for $p>1$. We shall return to this issue, however, in section 4.

$\begin{array}{ccc}\begin{array}{c}\text { Dimension } \\ (D \text { or } d)\end{array} & \begin{array}{c}\text { Minimal Spinor } \\ (M \text { or } m)\end{array} & \begin{array}{c}\text { Supersymmetry } \\ (N \text { or } n)\end{array} \\ 11 & 32 & 1 \\ 10 & 16 & 2,1 \\ 9 & 16 & 2,1 \\ 8 & 16 & 2,1 \\ 7 & 16 & 2,1 \\ 6 & 8 & 4,3,2,1 \\ 5 & 8 & 4,3,2,1 \\ 4 & 4 & 8, \ldots, 1 \\ 3 & 2 & 16, \ldots, 1 \\ 2 & 1 & 32, \ldots, 1\end{array}$

Table 1. Minimal spinor components and supersymmetries. 


\subsection{The Montonen-Olive conjecture}

Following Callan, Harvey and Strominger [36], we briefly review some general aspects of solitons. Two important features of solitons are the following [3]:

1) Most solitons are non-perturbative, i.e. they are solutions to nonlinear field equations which cannot be found by perturbation of the linearized field equations. Another non-perturbative feature is the fact that the mass per unit $p$-volume is inversely proportional to some power of a dimensionless coupling constant, so that the weak-coupling perturbative limit corresponds to the strong-coupling limit for the solitons. Since the classical solutions are non-perturbative, the quantum effects obtained from soliton interactions are also non-perturbative, and vanish to all orders in perturbation theory.

2) Most solitons are characterized by a conserved topological index which after quantization becomes a conserved quantum number. This in contrast to the conserved Noether charge associated with a continuous symmetry of the lagrangian.

In addition, soliton solutions typically depend on a finite number of parameters called moduli which act as coordinates on the moduli space of soliton solutions of fixed topological charge.

The simplest example of a soliton with these properties is the "kink" solution in $1+1$ spacetime dimensions. The solution to the Lagrangian

$$
\mathcal{L}=-\frac{1}{2} \partial_{\mu} \phi \partial^{\mu} \phi-U(\phi)
$$

with potential $U(\phi)=\lambda\left(\phi^{2}-m^{2} / \lambda\right)^{2} / 4$ and dimensionless coupling $g \equiv \lambda / m^{2}$ has conserved topological charge

$$
Q=\frac{\sqrt{g}}{2}(\phi(+\infty)-\phi(-\infty))
$$

$Q= \pm 1$ for a kink (anti-kink) in which $\phi$ varies from the minimum of $U$ at $\phi=\mp 1 / \sqrt{g}$ at $x=-\infty$ to the minimum at $\phi= \pm 1 / \sqrt{g}$ at $x=+\infty$. The energy (rest mass) of the kink is given by

$$
E=\int d x \frac{1}{2}\left(\phi^{\prime}\right)^{2}+U(\phi)=\frac{2 \sqrt{2}}{3} \frac{m}{g},
$$

so that the kink mass is proportional to $1 / \mathrm{g}$, and the solution is non-perturbative. It is straightforward to use the collective coordinates method [3] to separate out explicitly the dependence on the zero modes and to be left with a well defined perturbation theory for the non-zero modes. 
A supersymmetric version of the kink solution has Lagrangian

$$
\mathcal{L}=-\frac{1}{2}\left(\partial_{\mu} \phi\right)^{2}+\frac{1}{2} \bar{\psi} i \gamma^{\mu} \partial_{\mu} \psi-\frac{1}{2} V^{2}(\phi)-\frac{1}{2} V^{\prime}(\phi) \bar{\psi} \psi
$$

where $\psi$ is a Majorana fermion and $V=\lambda\left(\phi^{2}-a^{2}\right)$. This theory has two chiral supercharges given by

$$
Q_{ \pm}=\int d x\left(\dot{\phi} \pm \phi^{\prime}\right) \psi_{ \pm} \mp V(\phi) \psi_{\mp},
$$

where $\psi_{ \pm}$are the left- and right-handed components of $\psi$. The corresponding supersymmetry algebra is given by [37]

$$
Q_{+}^{2}=P_{+}, \quad Q_{-}^{2}=P_{-}, \quad\left\{Q_{+}, Q_{-}\right\}=T,
$$

with $P_{ \pm}=P_{0} \pm P_{1}$, and where the central term $T$ is esssentially the topological charge. The relation

$$
P_{+}+P_{-}=\left(Q_{+}+Q_{-}\right)^{2}-T=\left(Q_{+}-Q_{-}\right)^{2}+T
$$

implies a Bogomolnyi bound, $M \geq T / 2$, where $M$ is the rest mass. This bound is saturated precisely for those states $|s\rangle$ for which $\left(Q_{+} \pm Q_{-}\right)|s\rangle=0$, i.e. for states annihilated by some combination of the supersymmetry charges. In fact, demanding unbroken supersymmetry is equivalent to solving the "square root" of the equations of motion, and the fact that the kink solution is annihilated by one combination of the supercharges implies saturation of the Bogomolnyi bound. The other combination of supercharges does not annihilate the kink state but instead produces a fermion zero mode in the kink background. This follows from the fact that a supersymmetry variation of a solution to the bosonic equations of motion if it is nonzero produces a solution to the fermionic equations of motion in the bosonic background.

The property that half of the supercharges annihilate the classical solution leading to saturation of a Bogomolnyi bound, while the other half acting on the soliton produce fermion zero modes in the soliton background is found in most known examples of solitons in supersymmetric theories. As a result, searching for configurations which preserve some of the supercharges provides a shortcut to solving the full equations of motion since the resulting equations are typically first order as compared to the second order equations of motion. We will find this to be the case for the string soliton solutions discussed in this Report. 
We now turn to the magnetic monopole solution in Yang-Mills theory. The action for a Yang-Mills Higgs theory with gauge group $S U(2)$ and a Higgs field $\Phi$ in the adjoint representation is given by

$$
S=\int d^{4} x\left(-\frac{1}{4} \operatorname{Tr} F_{\mu \nu} F^{\mu \nu}-\frac{1}{2} \operatorname{Tr} D^{\mu} \Phi D_{\mu} \Phi-V(\Phi)\right),
$$

where $V$ has a minimum at $\left\langle\operatorname{Tr} \Phi^{2}\right\rangle=v^{2}$ which breaks $S U(2)$ down to $U(1)$. For a static configuration the energy is given by

$$
E=\int d^{3} x \operatorname{Tr}\left(B^{i} \pm D^{i} \Phi\right)^{2}+\int d^{3} x V(\Phi)+4 \pi v\left|Q_{M}\right|
$$

where $v$ is the asymptotic vacuum expectation value of $\Phi$. The energy thus satisfies a Bogomolnyi bound

$$
E \geq 4 \pi v\left|Q_{M}\right|
$$

where

$$
Q_{M}=\int \mathbf{B} \cdot d \mathbf{S}
$$

is the magnetic charge and $\mathbf{B}$ is the asymptotic value of the gauge invariant $U(1)$ field strength. The magnetic charge $Q_{M}$ is also related through the equations of motion to the element of $\pi_{2}(S U(2) / U(1))=Z$ which labels the topological charge carried by the Higgs field configuration. The Bogomolnyi bound is saturated if $V(\phi) \equiv 0$ and $B^{i}= \pm D^{i} \Phi$, which can be integrated to give explicit monopole solutions. The charge one solution was found in [38]; multi-monopole solutions are discussed in [39]. The universal formula for the classical mass of the particles of the theory is given by

$$
M^{2}=(4 \pi)^{2} v^{2}\left(Q_{E}^{2}+Q_{M}^{2}\right)
$$

where $Q_{E}$ and $Q_{M}$ are the electric and magnetic charges of the particle respectively. For monopoles (1.54) is simply the saturation of the Bogomolnyi bound. For massive gauge bosons, it is just the usual relation between the gauge boson mass and the Higgs vacuum expectation value.

The zero potential limit implies that the static force between two monopoles of like charge or between two gauge bosons of like charge vanishes. This is due to a cancellation between a repulsion due to photon (vector) exchange and an attraction due to massless Higgs boson (scalar) exchange [40]. This type of "zero-force condition" holds for all multisoliton solutions we consider in this Report. 
Based on ideas of [41], Montonen and Olive [42], conjectured the existence of a "dual" formulation of gauge theory in which the roles of gauge bosons and magnetic monopoles are exchanged. An example of this type of duality in $1+1$ spacetime dimensions is the the Thirring model - sine-Gordon duality, in which topological and Noether charges are exchanged.

In $3+1$ dimensions, however, monopoles have spin zero while gauge bosons have spin one, and the mass formula is probably not exact when quantum corrections are included, as the vanishing of the potential is not natural quantum mechanically. Embedding the theory in $N=2$ super Yang-Mills theory solves this problem, since then the charges $Q_{E}$ and $Q_{M}$ appear as central charges in the supersymmetry algebra as in the kink solution and the mass formula (1.54) is exact for supersymmetric states [37]. However, in $N=2$ the monopole states fill out a matter supermultiplet consisting of spin zero and spin one-half states. In order to construct monopoles with spin one it is necessary to extend the supersymmetry to $N=4$, the maximal allowable global supersymmetry in $3+1$ dimensions. This theory has a number of remarkable features. First, the structure of the fermion zero modes is such that the monopole supermultiplet now coincides with the gauge supermultiplet and includes states of spin 1, 1/2, and 0 [43]. Second, the scalar potential has exact flat directions due to supersymmetry and again the mass formula is exact. Finally, this theory is finite with vanishing beta-function, so that a duality which relates $g \rightarrow 1 / g$ can make sense quantum mechanically at all scales. Thus in this special theory all of the simple objections to the existence of the sort of duality suggested by Montonen and Olive disappear. Of course this is a far cry from showing that such a duality actually holds, but the evidence is suggestive enough that the idea is well worth pursuing.

Finally, the Montonen-Olive conjecture for more general gauge groups says that the dual gauge group should have a weight lattice dual to the weight lattice of the original group. It is tempting to speculate [26] that this is related to ten-dimensional heterotic string theory, where the gauge groups $S O(32) / Z_{2}$ and $E_{8} \times E_{8}$ with self-dual lattices are singled out by anomaly cancellation. 


\section{Strings and fivebranes in $\mathrm{D}=10$}

\subsection{The elementary string}

We begin by recalling the elementary string solution of [19]. We want to find a vacuumlike supersymmetric configuration with $D=2$ super-Poincare symmetry from the 3 -form version of $D=10, N=1$ supergravity theory. As usual, the fermionic fields should vanish for this configuration. We start by making an ansatz for the $D=10$ metric $g_{M N}, 2$-form $B_{M N}$ and dilaton $\phi(M=0,1, \cdots, 9)$ corresponding to the most general eight-two split invariant under $P_{2} \times S O(8)$, where $P_{2}$ is the $D=2$ Poincare group. We split the indices

$$
x^{M}=\left(x^{\mu}, y^{m}\right)
$$

where $\mu=0,1$ and $m=2, \cdots, 9$, and write the line element as

$$
d s^{2}=e^{2 A} \eta_{\mu \nu} d x^{\mu} d x^{\nu}+e^{2 B} \delta_{m n} d y^{m} d y^{n}
$$

and the two-form gauge field as

$$
B_{01}=-e^{C}
$$

All other components of $B_{M N}$ and all components of the gravitino $\psi_{M}$ and dilatino $\lambda$ are set zero. $P_{2}$ invariance requires that the arbitrary functions $A, B$ and $C$ depend only on $y^{m} ; S O(8)$ invariance then requires that this dependence be only through $y=\sqrt{\delta_{m n} y^{m} y^{n}}$. Similarly, our ansatz for the dilaton is

$$
\phi=\phi(y) .
$$

As we shall now show, the four arbitrary functions $A, B, C$, and $\phi$ are reduced to one by the requirement that the field configurations (2.2), (2.3) and (2.4) preserve some unbroken supersymmetry. In other words, there must exist Killing spinors $\varepsilon$ satisfying [19]

$$
\delta \psi_{M}=D_{M} \varepsilon+\frac{1}{96} e^{-\phi / 2}\left(\Gamma_{M}{ }^{N P Q}-9 \delta_{M}{ }^{N} \Gamma^{P Q}\right) H_{N P Q} \varepsilon=0
$$




$$
\delta \lambda=-\frac{1}{2 \sqrt{2}} \Gamma^{M} \partial_{M} \phi \varepsilon+\frac{1}{24 \sqrt{2}} e^{-\phi / 2} \Gamma^{M N P} H_{M N P} \varepsilon=0
$$

where

$$
H_{M N P}=3 \partial_{[M} A_{N P]} .
$$

Here $\Gamma_{A}$ are the $D=10$ Dirac matrices satisfying

$$
\left\{\Gamma_{A}, \Gamma_{B}\right\}=2 \eta_{A B}
$$

$A, B$ refer to the $D=10$ tangent space, $\eta_{A B}=(-,+, \cdots,+)$, and

$$
\Gamma_{A B \cdots C}=\Gamma_{[A} \Gamma_{B \cdots} \Gamma_{C]}
$$

thus $\Gamma_{A B}=\frac{1}{2}\left(\Gamma_{A} \Gamma_{B}-\Gamma_{B} \Gamma_{A}\right)$, etc. The $\Gamma^{\prime}$ 's with world-indices $P, Q, R, \cdots$ in $(2.5)$ and (2.6) have been converted using vielbeins $e_{M}{ }^{A}$. We make an eight-two split

$$
\Gamma_{A}=\left(\gamma_{\alpha} \otimes 1, \gamma_{3} \otimes \Sigma_{a}\right)
$$

where $\gamma_{\alpha}$ and $\Sigma_{a}$ are the $D=2$ and $D=8$ Dirac matrices, respectively. We also define

$$
\gamma_{3}=\gamma_{0} \gamma_{1}
$$

so that $\gamma_{3}^{2}=1$ and

$$
\Gamma_{9}=\Sigma_{2} \Sigma_{3} \cdots \Sigma_{9}
$$

so that $\Gamma_{9}^{2}=1$. The most general spinor consistent with $P_{2} \times S O(8)$ invariance takes the form

$$
\varepsilon(x, y)=\epsilon \otimes \eta
$$

where $\epsilon$ is a spinor of $S O(1,1)$ which may be further decomposed into chiral eigenstates via the projection operators $\left(1 \pm \gamma_{3}\right)$ and $\eta$ is an $S O(8)$ spinor which may further be decomposed into chiral eigenstates via the projection operators $\left(1 \pm \Gamma_{9}\right)$. The $N=1, D=$ 10 supersymmetry parameter is, however, subject to the ten-dimensional chirality condition

$$
\Gamma_{11} \varepsilon=\varepsilon,
$$

where $\Gamma_{11}=\gamma_{3} \otimes \Gamma_{9}$ and so the $D=2$ and $D=8$ chiralities are correlated.

Substituting the ansatz (2.2), (2.3) and (2.4) into (2.5) and (2.6) leads to the solution $[19]$

$$
\varepsilon=e^{3 \phi / 8} \epsilon_{0} \otimes \eta_{0}
$$


where $\epsilon_{0}$ and $\eta_{0}$ are constant spinors satisfying

$$
\left(1-\gamma_{3}\right) \epsilon_{0}=0, \quad\left(1-\Gamma_{9}\right) \eta_{0}=0
$$

and where

$$
\begin{aligned}
A & =\frac{3 \phi}{4}+c_{A}, \\
B & =-\frac{\phi}{4}+c_{B}, \\
C & =2 \phi+2 c_{A},
\end{aligned}
$$

where $c_{A}$ and $c_{B}$ are constants. If we insist that the metric is asymptotically Minkowskian, then

$$
c_{A}=-\frac{3 \phi_{0}}{4}, \quad c_{B}=\frac{\phi_{0}}{4}
$$

where $\phi_{0}$ is the value of $\phi$ at infinity i.e. the dilaton vev $\phi_{0}=\langle\phi\rangle$. The condition (2.16) means that one half of the supersymmetries are broken.

At this stage the four unknown functions $A, B, C$ and $\phi$ have been reduced to one by supersymmetry. To determine $\phi$, we must substitute the ansatz into the field equations which follow from the action $I_{10}$ (string) $+S_{2}$ where $I_{10}$ (string) is the bosonic sector of the 3 -form version of $D=10, N=1$ supergravity given by

$$
I_{10}(\text { string })=\frac{1}{2 \kappa^{2}} \int d^{10} x \sqrt{-g}\left(R-\frac{1}{2}(\partial \phi)^{2}-\frac{1}{2 \cdot 3 !} e^{-\phi} H^{2}\right),
$$

and $S_{2}$ is the string $\sigma$-model action. In (2.5), (2.6) and (2.19) we have employed the canonical choice of metric for which the gravitational action is the conventional EinsteinHilbert action. This metric is related to the metric appearing naturally in the string $\sigma$-model by

$$
g_{M N}(\text { string } \sigma \text {-model })=e^{\phi / 2} g_{M N}(\text { canonical }),
$$

which will be derived in the section 2.3. In canonical variables, therefore, the string $\sigma$ model action is given by

$$
\begin{gathered}
S_{2}=-T_{2} \int d^{2} \xi\left(\frac{1}{2} \sqrt{-\gamma} \gamma^{i j} \partial_{i} X^{M} \partial_{j} X^{N} g_{M N} e^{\phi / 2}-2 \sqrt{-\gamma}\right. \\
\left.+\frac{1}{2 !} \varepsilon^{i j} \partial_{i} X^{M} \partial_{j} X^{N} B_{M N}\right) .
\end{gathered}
$$


We have denoted the string tension by $T_{2}$. The supergravity field equations are

$$
\begin{aligned}
R^{M N} & -\frac{1}{2} g^{M N} R-\frac{1}{2}\left(\partial^{M} \phi \partial^{N} \phi-\frac{1}{2} g^{M N}(\partial \phi)^{2}\right) \\
& -\frac{1}{2 \cdot 2 !}\left(H_{P Q}^{M} H^{N P Q}-\frac{1}{6} g^{M N} H^{2}\right) e^{-\phi} \\
& =\kappa^{2} T^{M N}(\text { string }),
\end{aligned}
$$

where

$$
\begin{aligned}
& T^{M N}(\text { string })=-T_{2} \int d^{2} \xi \sqrt{-\gamma} \gamma^{i j} \partial_{i} X^{M} \partial_{j} X^{N} e^{\phi / 2} \frac{\delta^{10}(x-X)}{\sqrt{-g}}, \\
& \partial_{M}\left(\sqrt{-g} e^{-\phi} H^{M N P}\right) \\
& =2 \kappa^{2} T_{2} \int d^{2} \xi \varepsilon^{i j} \partial_{i} X^{N} \partial_{j} X^{P} \delta^{10}(x-X), \\
& \phi+\frac{1}{2 \cdot 3 !} e^{-\phi} H^{2}=\frac{\kappa^{2} T_{2}}{2} \int d^{6} \xi \sqrt{-\gamma} \gamma^{i j} \partial_{i} X^{M} \partial_{j} X^{N} g_{M N} e^{\phi / 2} \frac{\delta^{10}(x-X)}{\sqrt{-g}}
\end{aligned}
$$

Furthermore, the string field equations are

$$
\begin{aligned}
& \partial_{i}\left(\sqrt{-\gamma} \gamma^{i j} \partial_{j} X^{N} g_{M N} e^{\phi / 2}\right)-\frac{1}{2} \sqrt{-\gamma} \gamma^{i j} \partial_{i} X^{N} \partial_{j} X^{P} \partial_{M}\left(g_{N P} e^{\phi / 2}\right) \\
& -\frac{1}{2} \varepsilon^{i j} \partial_{i} X^{N} \partial_{j} X^{P} H_{M N P}=0
\end{aligned}
$$

and

$$
\gamma_{i j}=\partial_{i} X^{M} \partial_{j} X^{N} g_{M N} e^{\phi / 2}
$$

To solve these coupled supergravity-string equations we make the static gauge choice

$$
X^{\mu}=\xi^{\mu}, \quad \mu=0,1
$$

and the ansatz

$$
X^{m}=Y^{m}=\text { constant }, \quad m=2, \ldots, 9 .
$$

As an example, let us now substitute (2.17), (2.28) and (2.29) into the 2-form equation (2.24). We find

$$
\delta^{m n} \partial_{m} \partial_{n} e^{-2 \phi}=-2 \kappa^{2} T_{2} e^{-\phi_{0} / 2} \delta^{8}(y)
$$

and hence

$$
e^{-2 \phi}=e^{-2 \phi_{0}}\left(1+\frac{k_{2}}{y^{6}}\right)
$$


where the constant $k_{2}$ is given by

$$
k_{2} \equiv \frac{\kappa^{2} T_{2}}{3 \Omega_{7}} e^{3 \phi_{0} / 2}
$$

and $\Omega_{n}$ is the volume of the unit $n$-sphere $S^{n}$. One may verify that all the field equations (2.22) to (2.27) are reduced to a single equation (2.30) by using (2.18), (2.28), (2.29) and the expressions for the Ricci tensor $R^{M N}$ and Ricci scalar $R$ in terms of $A$ and $B$. For future reference, we shall compute these for arbitrary worldvolume dimension $d$ and spacetime dimension $D$. The ansatz is

$$
d s^{2}=e^{2 A} \eta_{\mu \nu} d x^{\mu} d x^{\nu}+e^{2 B} \delta_{m n} d y^{m} d y^{n}
$$

where $\mu=0,1, \cdots, d-1 ; m=d, d+1, \cdots, D-1$ and $A=A(y), B=B(y)$. For the above metric, we have

$$
\begin{gathered}
R_{\mu \nu}=-\eta_{\mu \nu} \delta^{m n} e^{2(A-B)}\left(\partial_{m} \partial_{n} A+d \partial_{m} A \partial_{n} A+\tilde{d} \partial_{m} A \partial_{n} B\right) \\
R_{m n}=-\tilde{d} \partial_{m} \partial_{n} B-\delta_{m n} \delta^{k l} \partial_{k} \partial_{l} B-d \partial_{m} \partial_{n} A \\
+d\left(\partial_{m} A \partial_{n} B+\partial_{n} A \partial_{m} B-\delta_{m n} \delta^{k l} \partial_{k} A \partial_{l} B\right)-d \partial_{m} A \partial_{n} B \\
+\tilde{d} \partial_{m} B \partial_{n} B-\tilde{d} \delta_{m n} \delta^{k l} \partial_{k} B \partial_{l} B \\
R=e^{-2 B}\left[-2 d \delta^{m n} \partial_{m} \partial_{n} A-d(d+1) \delta^{m n} \partial_{m} A \partial_{n} A\right. \\
-2 d \tilde{d} \delta^{m n} \partial_{m} A \partial_{n} B \\
\left.-2(\tilde{d}+1) \delta^{m n} \partial_{m} \partial_{n} B-\tilde{d}(\tilde{d}+1) \delta^{m n} \partial_{m} B \partial_{n} B\right]
\end{gathered}
$$

where $\tilde{d} \equiv D-d-2$.

Having established that the supergravity configuration preserves half the supersymmetries, we must also verify that the string configuration (2.28) and (2.29) also preserve these supersymmetries. As discussed in [44], the criterion is that in addition to the existence of Killing spinors satisfying (2.5) and (2.6) we must also have

$$
(1-\Gamma) \varepsilon=0
$$

where the choice of sign is dictated by the choice of the sign in the Wess-Zumino term in $(2.21)$, and where

$$
\Gamma \equiv \frac{1}{2 ! \sqrt{-\gamma}} \varepsilon^{i j} \partial_{i} X^{M} \partial_{j} X^{N} \Gamma_{M N}
$$


Since $\Gamma^{2}=1$ and $\operatorname{tr} \Gamma=0, \frac{1}{2}(1 \pm \Gamma)$ act as projection operators. From (2.28) and (2.29) we see that

$$
\Gamma=\gamma_{3} \otimes 1
$$

and hence (2.37) is satisfied as a consequence of (2.16). Equation (2.37) explains, from a string point of view, why the solutions we have found preserve just half the supersymmetries. It originates from the fermionic $\kappa$-symmetry of the superstring action. The fermionic zero-modes on the worldvolume are just the Goldstone fermions associated with the broken supersymmetry $[8,18]$.

As shown in [19] the elementary string solution saturates a Bogolmol'nyi bound for the mass per unit length

$$
\mathcal{M}_{2}=\int d^{8} y \theta_{00}
$$

where $\theta_{M N}$ is the total energy-momentum pseudotensor of the combined gravity-matter system. One finds

$$
\kappa \mathcal{M}_{2} \geq \frac{1}{\sqrt{2}}\left|e_{2}\right| e^{\phi_{0} / 2}
$$

where $e_{2}$ is the Noether "electric" charge whose conservation follows the equation of motion of the 2-form (2.24), namely

$$
e_{2}=\frac{1}{\sqrt{2} \kappa} \int_{S^{7}} e^{-\phi *} H
$$

where ${ }^{*}$ denotes the Hodge dual using the canonical metric and the integral is over an asymptotic seven-sphere surrounding the string. We find for our solution that

$$
\mathcal{M}_{2}=e^{\phi_{0} / 2} T_{2}
$$

and

$$
e_{2}=\sqrt{2} \kappa T_{2}
$$

Hence the bound is saturated. This provides another way, in addition to unbroken supersymmetry, to understand the stability of the solution.

A straightforward generalization to exact, stable multi-string configurations can be obtained by replacing the single string $\sigma$-model source by a superposition of $N$ string sources. The resulting solution is a linear superposition of solutions to (2.30)

$$
e^{-2 \phi}=e^{-2 \phi_{0}}\left[1+\sum_{\ell} \frac{k_{2}}{\left|\vec{y}-\vec{y}_{\ell}\right|^{6}}\right] \text {, }
$$


where $\vec{y}_{\ell}$ corresponds to the position of each string. The ability to superpose solutions of this kind is a well-known phenomenon in soliton and instanton physics and goes by the name of the "no-force condition". In the present context, it means that the gravitationaldilatonic attractive force acting on each of the strings is exactly cancelled by an equal but repulsive force from the 2 -form. This effect is also present in other elementary and solitonic solutions and is closely related both to the saturation of the Bogomol'nyi bound and to the existence of unbroken supersymmetry. In the supersymmetric context the no-force condition is sometimes called "antigravity". To see this explicitly, consider the multi-string configurations (2.45) with, for example, $N$ strings as sources. In general, we do not have the transverse $S O(8)$ symmetry, but we still have the $P_{2}$ symmetry for the configurations (2.45). Let each string with label $l$ satisfy $X^{\mu}(l)=\xi^{\mu}$ so that, in particular, they all have the same orientation. The lagrangian for this $N$-string configuration in the fields of the sources given by (2.2) and (2.3) is, from (2.21),

$$
\mathcal{L}_{2}=-T_{2}\left[\sqrt{-\operatorname{det}\left(e^{2 A+\phi / 2} \eta_{i j}+e^{2 B+\phi / 2} \partial_{i} Y^{m}(l) \partial_{j} Y_{m}(l)\right)}-e^{C}\right]
$$

corresponding to a potential

$$
V=T_{2}\left(e^{2 A+\phi / 2}-e^{C}\right)
$$

But this vanishes by the supersymmetry conditions (2.17). On the other hand, if the $N$ strings had the opposite orientation, and hence the opposite sign $e_{2}$, then the sign change in the Wess-Zumino term in (2.46) would result in a net attractive force and therefore the corresponding configurations cannot be stable.

\subsection{The solitonic fivebrane}

The elementary string discussed above is a solution of the coupled field-string system with action $I_{10}$ (string) $+S_{2}$. As such it exhibits $\delta$-function singularities at $y=0$. It is characterized by a non-vanishing Noether electric charge $e_{2}$. By contrast, we now wish to find a solitonic fivebrane, corresponding to a solution of the source free equations resulting from $I_{10}$ (string) alone and which will be characterized by a non-vanishing topological "magnetic" charge $g_{6}$.

To this end, we now make an ansatz invariant under $P_{6} \times S O(4)$. Hence we write (2.1) and (2.2) as before where now $\mu=0,1 \ldots 5$ and $m=6,7,8,9$. The ansatz for the 
antisymmetric tensor, however, will now be made on the field strength rather than on the potential. From section 2.1 we recall that a non-vanishing electric charge corresponds to

$$
\frac{1}{\sqrt{2} \kappa} e^{-\phi *} H=e_{2} \varepsilon_{7} / \Omega_{7}
$$

where $\varepsilon_{n}$ is the volume form on $S^{n}$. Accordingly, to obtain a non-vanishing magnetic charge, we make the ansatz

$$
\frac{1}{\sqrt{2} \kappa} H=g_{6} \varepsilon_{3} / \Omega_{3}
$$

Since this is an harmonic form, $H$ can no longer be written globally as the curl of $B$, but it satisfies the Bianchi identity. It is now not difficult to show that all the field equations are satisfied. The solution is given by

$$
\begin{aligned}
e^{2 \phi} & =e^{2 \phi_{0}}\left(1+\frac{k_{6}}{y^{2}}\right), \\
d s^{2} & =e^{-\left(\phi-\phi_{0}\right) / 2} \eta_{\mu \nu} d x^{\mu} d x^{\nu}+e^{3\left(\phi-\phi_{0}\right) / 2} \delta_{m n} d y^{m} d y^{n}, \\
H & =2 k_{6} e^{\phi_{0} / 2} \varepsilon_{3},
\end{aligned}
$$

where $\mu, \nu=0,1, \ldots, 5, m, n=6,7,8,9$ and where

$$
k_{6}=\frac{\kappa g_{6}}{\sqrt{2} \Omega_{3}} e^{-\phi_{0} / 2}
$$

It follows that the mass per unit 5-volume now saturates a bound involving the magnetic charge

$$
\mathcal{M}_{6}=\frac{1}{\sqrt{2}}\left|g_{6}\right| e^{-\phi_{0} / 2}
$$

Note that the $\phi_{0}$ dependence is such that $\mathcal{M}_{6}$ is large for small $\mathcal{M}_{2}$ and vice-versa.

The electric charge of the elementary solution and the magnetic charge of the soliton solution obey a Dirac quantization rule $[27,28]$

$$
e_{2} g_{6}=2 \pi n, \quad n=\text { integer }
$$

and hence from $(2.44)$

$$
g_{6}=2 \pi n / \sqrt{2} \kappa T_{2} .
$$




\subsection{String/fivebrane duality}

The 3-form version of $D=10, N=1$ supergravity admits the elementary string as a solution [19]. In this and the next section we shall show that the 7 -form version admits the elementary fivebrane as a solution [29]. The elementary string can also be interpreted as a magnetic-like solution of the 7 -form version, as can the elementary fivebrane for the 3 -form version. This suggests that the heterotic string and the heterotic fivebrane may be dual to each other in the sense that they are equivalent descriptions of the same underlying physical theory. In the dual formulation, the metric $g_{M N}$ and the dilaton $\phi$ are the same, whereas the 7 -form field strength $K$ of the fivebrane is dual to the 3 -form field strength of the string $H$. More precisely,

$$
H=e^{\phi *} K,
$$

where ${ }^{*}$ denotes the Hodge dual using the canonical metric, so that the field equation of the 3 -form becomes the Bianchi identity of the 7 -form and vice-versa. Hence

$$
I_{10}(\text { fivebrane })=\frac{1}{2 \kappa^{2}} \int d^{10} x \sqrt{-g}\left(R-\frac{1}{2}(\partial \phi)^{2}-\frac{1}{2 \cdot 7 !} e^{\phi} K^{2}\right),
$$

where $K$ is the curl of the 6 -form $A$ :

$$
K=d A .
$$

String/fivebrane duality will tell us that the fivebrane loop coupling constant $\mathrm{g}_{6}$ is given by the inverse cube root of the string loop coupling constant $\mathrm{g}_{2}$,

$$
\mathrm{g}_{6}=\mathrm{g}_{2}^{-1 / 3}=e^{-\phi_{0} / 3},
$$

which implies that the strongly coupled heterotic string corresponds to the weakly coupled fivebrane, and vice versa. We will now derive (2.58) and the following relations between the canonical gravitational metric and the metrics which appear naturally in the string and fivebrane $\sigma$-models:

$$
g_{M N}(\text { canonical })=e^{-\phi / 2} g_{M N}(\text { string })=e^{\phi / 6} g_{M N}(\text { fivebrane }) .
$$

In general, we have

$$
g_{M N}(\text { string })=\Omega_{s}(\phi) g_{M N}(\text { canonical }),
$$

and

$$
g_{M N}(\text { fivebrane })=\Omega_{f}(\phi) g_{M N}(\text { canonical })
$$


The corresponding lowest-order $\sigma$-model actions written in terms of the canonical metric are given by

$$
S_{2}=-T_{2} \int d^{2} \xi\left(\frac{1}{2} \sqrt{-\gamma} \gamma^{i j} \partial_{i} X^{M} \partial_{j} X^{N} \Omega_{s}(\phi) g_{M N}+\frac{1}{2} \varepsilon^{i j} \partial_{i} X^{M} \partial_{j} X^{N} B_{M N}\right),
$$

for the string and

$$
\begin{aligned}
S_{6}=-T_{6} \int d^{6} \xi( & \frac{1}{2} \sqrt{-\gamma} \gamma^{i j} \partial_{i} X^{M} \partial_{j} X^{N} \Omega_{f}(\phi) g_{M N}-2 \sqrt{-\gamma} \\
& \left.+\frac{1}{6 !} \varepsilon^{i j k l m n} \partial_{i} X^{M} \partial_{j} X^{N} \partial_{k} X^{O} \partial_{l} X^{P} \partial_{m} X^{Q} \partial_{n} X^{R} A_{M N O P Q R}\right)
\end{aligned}
$$

for the fivebrane. Note that in this case

$$
\gamma_{i j}=\partial_{i} X^{M} \partial_{j} X^{N} \Omega_{f}(\phi) g_{M N}
$$

To lowest order, the actions $I_{10}$ (string) and $S_{2}$ describe the same string (for example, the elementary string), and the actions $I_{10}$ (fivebrane) and $S_{6}$ describe the same fivebrane. Moreover, string/fivebrane duality implies that either $I_{10}$ (string) or $I_{10}$ (fivebrane) describes not only the string but also the fivebrane by using (2.55). Therefore, we would expect that both $I_{10}$ (string) and $I_{10}$ (fivebrane) should scale in the same way as both $S_{2}$ and $S_{6}$ under the following constant rescalings:

$$
\begin{aligned}
g_{M N} & \rightarrow \alpha g_{M N}, \\
e^{\phi} & \rightarrow \beta e^{\phi}, \\
B_{M N} & \rightarrow \lambda^{2} B_{M N}, \\
A_{M N O P Q R} & \rightarrow \sigma^{6} A_{M N O P Q R} .
\end{aligned}
$$

From the above requirements, each of the actions $I_{10}$ (string), $I_{10}$ (fivebrane), $S_{2}$ and $S_{6}$ should also scale homogeneously. It follows from (2.62) that

$$
S_{2} \rightarrow \lambda^{2} S_{2}
$$

and

$$
\Omega_{s}(\phi) \rightarrow \frac{\lambda^{2}}{\alpha} \Omega_{s}(\phi)
$$

from (2.63) that

$$
S_{6} \rightarrow \sigma^{6} S_{6},
$$


and

$$
\Omega_{f}(\phi) \rightarrow \frac{\sigma^{2}}{\alpha} \Omega_{f}(\phi),
$$

from (2.19) that

$$
I_{10}(\text { string }) \rightarrow \alpha^{4} I_{10} \text { (string) }
$$

and

$$
\lambda^{4}=\beta \alpha^{2},
$$

and from (2.56) that

$$
\left.I_{10}(\text { fivebrane }) \rightarrow \alpha^{4} I_{10} \text { (fivebrane }\right)
$$

and

$$
\alpha^{6}=\beta \sigma^{12}
$$

As expected, $I_{10}$ (string) scales the same way as $I_{10}$ (fivebrane). Combining either (2.73) or (2.75) with (2.69) and (2.71), we obtain

$$
\alpha^{4}=\lambda^{2} \sigma^{6}
$$

Hence, by using $(2.74),(2.76)$ and $(2.77)$, we obtain

$$
\alpha=\lambda^{1 / 2} \sigma^{3 / 2}
$$

and

$$
\beta=\lambda^{3} \sigma^{-3}
$$

Substituting (2.79) into (2.66), and (2.78) into (2.65), (2.70) and (2.72), respectively, we obtain

$$
\begin{gathered}
e^{\phi} \rightarrow \lambda^{3} \sigma^{-3} e^{\phi}, \\
g_{M N} \rightarrow \lambda^{1 / 2} \sigma^{3 / 2} g_{M N}, \\
\Omega_{s}(\phi) \rightarrow\left(\lambda^{3} \sigma^{-3}\right)^{1 / 2} \Omega_{s}(\phi),
\end{gathered}
$$

and

$$
\Omega_{f}(\phi) \rightarrow\left(\lambda^{3} \sigma^{-3}\right)^{-1 / 6} \Omega_{f}(\phi)
$$

Comparing (2.82) and (2.83) with (2.80), we immediately have

$$
\Omega_{s}(\phi)=e^{\phi / 2}, \quad \Omega_{f}(\phi)=e^{-\phi / 6},
$$


which implies (2.59). This result agrees with what one obtains in string theory by setting the $\beta$-functions of $g_{M N}, B_{M N}$ and $\phi$ to zero, i.e. by using string worldsheet conformal invariance.

We are now ready to derive (2.58). This can be achieved simply by writing $I_{10}$ (string) and $I_{10}$ (fivebrane) in terms of string and fivebrane metrics given in (2.59), respectively. We obtain for the string action

$$
I_{10}(\text { string })=\frac{1}{2 \kappa^{2}} \int d^{10} x \sqrt{-g} e^{-2 \phi}\left(R+4(\partial \phi)^{2}-\frac{1}{2 \cdot 3 !} H^{2}\right),
$$

where the common factor $e^{-2 \phi}$ for each term implies that the string loop counting parameter $\mathrm{g}_{2}$ is given by

$$
\mathrm{g}_{2}=e^{\phi_{0}}
$$

For the fivebrane action,

$$
I_{10}(\text { fivebrane })=\frac{1}{2 \kappa^{2}} \int d^{10} x \sqrt{-g} e^{2 \phi / 3}\left(R-\frac{1}{2 \cdot 7 !} K^{2}\right)
$$

and an analogous situation arises, namely a common factor $e^{2 \phi / 3}$ in front of each term. Although we have yet to construct the heterotic fivebrane action and quantize it, string/fivebrane duality tells that if heterotic string theory is a sensible theory, then so is heterotic fivebrane theory. So we would expect that the action (2.87) would be the field-theory limit of the heterotic fivebrane. In analogy with the case of (2.85), the common factor of (2.87) suggests that the fivebrane loop counting parameter $\mathrm{g}_{6}$ is given by

$$
\mathrm{g}_{6}=e^{-\phi_{0} / 3}
$$

Combining (2.86) and (2.88) we obtain (2.58).

\subsection{The elementary fivebrane}

In this section, we rederive the fivebrane soliton as an elementary electric-like singular solution, the "elementary fivebrane" [29] of the 7-form version of $D=10, N=1$ supergravity, which is the analog of a static electric-like singular "elementary string" solution [19] of the 3-form version.

We want to find a vacuum-like supersymmetric configuration with $D=6$ superPoincare symmetry from the 7 -form version of $D=10, N=1$ supergravity theory. As 
usual, the fermionic fields should vanish for this configuration. We start by making an ansatz for the $D=10$ metric $g_{M N}, 6$-form $A_{M N O P Q R}$ and dilaton $\phi(M=0,1, \cdots, 9)$ corresponding to the most general six-four split invariant under $P_{6} \times S O(4)$, where $P_{6}$ is the $D=6$ Poincare group. We split the indices

$$
x^{M}=\left(x^{\mu}, y^{m}\right)
$$

where $\mu=0, \cdots, 5$ and $m=6, \cdots, 9$, and write the line element as

$$
d s^{2}=e^{2 A} \eta_{\mu \nu} d x^{\mu} d x^{\nu}+e^{2 B} \delta_{m n} d y^{m} d y^{n}
$$

and the six-form gauge field as

$$
A_{\mu \nu \rho \sigma \lambda \tau}=-\frac{1}{{ }^{6} g} \varepsilon_{\mu \nu \rho \sigma \lambda \tau} e^{C}
$$

where ${ }^{6} g$ is the determinant of $g_{\mu \nu}, \varepsilon_{\mu \nu \rho \sigma \lambda \tau} \equiv g_{\mu \alpha} g_{\nu \beta} g_{\rho \gamma} g_{\sigma \delta} g_{\lambda \epsilon} g_{\tau \xi} \varepsilon^{\alpha \beta \gamma \delta \epsilon \xi}$ and $\varepsilon^{012345}=1$, i.e. $A_{012345}=-e^{C}$. All other components of $A_{M N O P Q R}$ and all components of the gravitino $\psi_{M}$ and dilatino $\lambda$ are set zero. $P_{6}$ invariance requires that the arbitrary functions $A, B$ and $C$ depend only on $y^{m} ; S O(4)$ invariance then requires that this dependence be only through $y=\sqrt{\delta_{m n} y^{m} y^{n}}$. Similarly, our ansatz for the dilaton is

$$
\phi=\phi(y)
$$

As we shall now show, the four arbitrary functions $A, B, C$, and $\phi$ are reduced to one by the requirement that the field configurations (2.90), (2.91) and (2.92) preserve some unbroken supersymmetry. In other words, there must exist Killing spinors $\varepsilon$ satisfying

$$
\begin{gathered}
\delta \psi_{M}=D_{M} \varepsilon+\frac{1}{2 \cdot 8 !} e^{\phi / 2}\left(3 \Gamma_{M}^{N O P Q R S T}-7 \delta_{M}^{N} \Gamma^{O P Q R S T}\right) K_{N O P Q R S T} \varepsilon=0 \\
\delta \lambda=-\frac{1}{2 \sqrt{2}} \Gamma^{M} \partial_{M} \phi \varepsilon-\frac{1}{2 \cdot 2 \sqrt{2} \cdot 7 !} e^{\phi / 2} \Gamma^{M N O P Q R S} K_{M N O P Q R S} \varepsilon=0
\end{gathered}
$$

where

$$
K_{M N O P Q R S}=7 \partial_{[M} A_{N O P Q R S]}
$$

We make a six-four split

$$
\Gamma_{A}=\left(\gamma_{\alpha} \otimes 1, \gamma_{7} \otimes \Sigma_{a}\right)
$$


where $\gamma_{\alpha}$ and $\Sigma_{a}$ are the $D=6$ and $D=4$ Dirac matrices, respectively. We also define

$$
\gamma_{7}=\gamma_{0} \gamma_{1} \gamma_{2} \gamma_{3} \gamma_{4} \gamma_{5}
$$

so that $\gamma_{7}^{2}=1$ and

$$
\Gamma_{5}=\Sigma_{6} \Sigma_{7} \Sigma_{8} \Sigma_{9}
$$

so that $\Gamma_{5}^{2}=1$. The most general spinor consistent with $P_{6} \times S O(4)$ invariance takes the form

$$
\varepsilon(x, y)=\epsilon \otimes \eta
$$

where $\epsilon$ is a spinor of $S O(1,5)$ which may be further decomposed into chiral eigenstates via the projection operators $\left(1 \pm \gamma_{7}\right)$ and $\eta$ is an $S O(4)$ spinor which may further be decomposed into chiral eigenstates via the projection operators $\left(1 \pm \Gamma_{5}\right)$. The $N=1, D=$ 10 supersymmetry parameter is, however, subject to the ten-dimensional chirality condition

$$
\tilde{\Gamma}_{11} \varepsilon=\varepsilon
$$

where $\Gamma_{11}=\gamma_{7} \otimes \Gamma_{5}$ and so the $D=4$ and $D=6$ chiralities are correlated.

In our background (2.90), (2.91) and (2.92), the transformation rules (2.93) and (2.94) reduce to

$$
\begin{aligned}
\partial_{\mu} \varepsilon & -\frac{1}{8} \gamma_{\mu} \Sigma^{m}\left[\frac{1}{2} e^{\phi / 2-6 A} \partial_{m} e^{C}-4 \gamma_{7} \partial_{m} A\right] \varepsilon=0, \\
\partial_{m} \varepsilon & +\frac{1}{2}\left(\Sigma_{m} \Sigma^{n} \partial_{n} B-\partial_{m} B\right) \varepsilon \\
& +\frac{1}{16} e^{\phi / 2-6 A} \gamma_{7}\left(3 \Sigma_{m} \Sigma^{n} \partial_{n} e^{C}+4 \partial_{m} e^{C}\right) \varepsilon=0,
\end{aligned}
$$

and

$$
\Sigma^{m}\left(\frac{1}{2} e^{\phi / 2-6 A} \partial_{m} e^{C}+\gamma_{7} \partial_{m} \phi\right) \varepsilon=0 .
$$

Note that the $\gamma_{\mu}$ and $\Sigma_{m}$ carry world indices. Hence we find that [29]

$$
\varepsilon=e^{-\phi / 8} \epsilon_{0} \otimes \eta_{0}
$$

where $\epsilon_{0}$ and $\eta_{0}$ are constant spinors satisfying

$$
\left(1-\gamma_{7}\right) \epsilon_{0}=0, \quad\left(1-\Gamma_{5}\right) \eta_{0}=0
$$


and where

$$
\begin{aligned}
A & =-\frac{\phi}{4}+c_{A}, \\
B & =\frac{3 \phi}{4}+c_{B}, \\
C & =-2 \phi+6 c_{A},
\end{aligned}
$$

where $c_{A}$ and $c_{B}$ are constants. If we insist that the metric is asymptotically Minkowskian, then

$$
c_{A}=+\frac{\phi_{0}}{4}, \quad c_{B}=-\frac{3 \phi_{0}}{4} .
$$

The condition (2.105) means that one half of the supersymmetries are broken.

At this stage the four unknown functions $A, B, C$ and $\phi$ have been reduced to one by supersymmetry. To determine $\phi$, we must substitute the ansatz into the field equations which follow from the action $I_{10}$ (fivebrane) $+S_{6}$. In canonical variables, the supergravity field equations are

$$
\begin{aligned}
R^{M N} & -\frac{1}{2} g^{M N} R-\frac{1}{2}\left(\partial^{M} \phi \partial^{N} \phi-\frac{1}{2} g^{M N}(\partial \phi)^{2}\right) \\
& -\frac{1}{2 \cdot 6 !}\left(K^{M} \text { OPQRST } K^{N O P Q R S T}-\frac{1}{14} g^{M N} K^{2}\right) e^{\phi} \\
& =\kappa^{2} T^{M N} \text { (fivebrane) }
\end{aligned}
$$

where

$$
\begin{gathered}
T^{M N}(\text { fivebrane })=-T_{6} \int d^{6} \xi \sqrt{-\gamma} \gamma^{i j} \partial_{i} X^{M} \partial_{j} X^{N} e^{-\phi / 6} \frac{\delta^{10}(x-X)}{\sqrt{-g}}, \\
\partial_{M}\left(\sqrt{-g} e^{\phi} K^{M N O P Q R S}\right) \\
=2 \kappa^{2} T_{6} \int d^{6} \xi \varepsilon^{i j k l m n} \partial_{i} X^{N} \partial_{j} X^{O} \partial_{k} X^{P} \partial_{l} X^{Q} \partial_{m} X^{R} \partial_{n} X^{S} \delta^{10}(x-X), \\
-\square \phi+\frac{1}{2 \cdot 7 !} e^{\phi} K^{2}=\frac{\kappa^{2} T_{6}}{6} \int d^{6} \xi \sqrt{-\gamma} \gamma^{i j} \partial_{i} X^{M} \partial_{j} X^{N} g_{M N} e^{-\phi / 6} \frac{\delta^{10}(x-X)}{\sqrt{-g}} .
\end{gathered}
$$

Furthermore, the fivebrane field equations are

$$
\begin{aligned}
& \partial_{i}\left(\sqrt{-\gamma} \gamma^{i j} \partial_{j} X^{N} g_{M N} e^{-\phi / 6}\right)-\frac{1}{2} \sqrt{-\gamma} \gamma^{i j} \partial_{i} X^{N} \partial_{j} X^{P} \partial_{M}\left(g_{N P} e^{-\phi / 6}\right) \\
& -\frac{1}{6 !} \varepsilon^{i j k l m n} \partial_{i} X^{N} \partial_{j} X^{O} \partial_{k} X^{P} \partial_{l} X^{Q} \partial_{m} X^{R} \partial_{n} X^{S} K_{M N O P Q R S}=0
\end{aligned}
$$

and

$$
\gamma_{i j}=\partial_{i} X^{M} \partial_{j} X^{N} g_{M N} e^{-\phi / 6} .
$$


To solve these coupled supergravity-fivebrane equations we make the static gauge choice

$$
X^{\mu}=\xi^{\mu}, \quad \mu=0,1,2,3,4,5
$$

and the ansatz

$$
X^{m}=Y^{m}=\text { constant }, \quad m=6,7,8,9 .
$$

As an example, let us now substitute (2.106), (2.114) and (2.115) into the 6-form equation (2.110). We find

$$
\delta^{m n} \partial_{m} \partial_{n} e^{2 \phi}=-2 \kappa^{2} T_{6} e^{3 \phi_{0} / 2} \delta^{4}(y),
$$

and hence

$$
e^{2 \phi}=e^{2 \phi_{0}}\left(1+\frac{k_{6}}{y^{2}}\right)
$$

where the constant $k_{6}$ is now given by

$$
k_{6} \equiv \frac{\kappa^{2} T_{6}}{\Omega_{3}} e^{-\phi_{0} / 2} .
$$

One may verify that all the field equations (2.108) to (2.113) are reduced to a single equation (2.116) by using (2.107), (2.114), (2.115) and the expressions for the Ricci tensor $R^{M N}$ and Ricci scalar $R$ in terms of $A$ and $B$ given in the general case above.

Having established that the supergravity configuration preserves half the supersymmetries, we must also verify that the fivebrane configuration (2.114) and (2.115) also preserve these supersymmetries. As discussed in [44], the criterion is that in addition to the existence of Killing spinors satisfying (2.93) and (2.94) we must also have

$$
(1-\tilde{\Gamma}) \varepsilon=0
$$

where the choice of sign is dictated by the choice of the sign in the Wess-Zumino term in (2.63), and where

$$
\tilde{\Gamma} \equiv \frac{1}{6 ! \sqrt{-\gamma}} \varepsilon^{i j k l m n} \partial_{i} X^{M} \partial_{j} X^{N} \partial_{k} X^{O} \partial_{l} X^{P} \partial_{m} X^{Q} \partial_{n} X^{R} \Gamma_{M N O P Q R} .
$$

Since $\tilde{\Gamma}^{2}=1$ and $\operatorname{tr} \tilde{\Gamma}=0, \frac{1}{2}(1 \pm \tilde{\Gamma})$ act as projection operators. From (2.114) and (2.115) we see that

$$
\tilde{\Gamma}=\gamma_{7} \otimes 1,
$$

and hence (2.119) is satisfied as a consequence of (2.105). (2.119) explains, from a fivebrane point of view, why the solutions we have found preserve just half the supersymmetries. It 
originates from the fermionic $\kappa$-symmetry of the superfivebrane action. Again the fermionic zero-modes on the worldvolume are just the Goldstone fermions associated with the broken supersymmetry $[8,18]$.

Using the same method as in section 2.1, we may also establish a bound for the mass per unit area of the fivebrane

$$
\mathcal{M}_{6}=\int d^{4} y \theta_{00}
$$

where $\theta_{M N}$ is the total energy-momentum pseudotensor of the combined gravity-matter system. One finds

$$
\kappa \mathcal{M}_{6} \geq \frac{1}{\sqrt{2}}\left|\tilde{e}_{6}\right| e^{-\phi_{0} / 2}
$$

where $\tilde{e}_{6}$ is the Noether charge whose conservation follows the equation of motion of the 6-form (2.110), namely

$$
\tilde{e}_{6}=\frac{1}{\sqrt{2} \kappa} \int_{S^{3}} e^{\phi *} K,
$$

where ${ }^{*}$ denotes the Hodge dual and the integral is over an asymptotic three-sphere surrounding the fivebrane. We find for our solution that

$$
\mathcal{M}_{6}=e^{-\phi_{0} / 2} T_{6}
$$

and

$$
\tilde{e}_{6}=\sqrt{2} \kappa T_{6}
$$

Hence the bound is saturated. Once again, this provides another way, in addition to unbroken supersymmetry, to understand the stability of the solution.

As in the case of the elementary string, there is a straightforward generalization to exact, stable multi-fivebrane configurations by linear superposition of

$$
e^{2 \phi}=e^{2 \phi_{0}}\left[1+\sum_{\ell} \frac{k_{6}}{\left|\vec{y}-\vec{y}_{\ell}\right|^{2}}\right]
$$

where $\vec{y}_{\ell}$ corresponds to the position of each fivebrane. In this case the no-force condition results from the cancellation of the gravitational-dilatonic attractive force with the repulsive force from the 6 -form. To see this explicitly, consider the multi-fivebrane configurations (2.127) with, for example, $N$ fivebranes as sources. In general, we do not have the transverse $S O(4)$ symmetry, but we still have the $P_{6}$ symmetry for the configurations (2.127). Let each fivebrane with label $l$ satisfy $X^{\mu}(l)=\xi^{\mu}$ so that, in particular, they all 
have the same orientation. The lagrangian for this $N$-fivebrane in the fields of the sources given by (2.90) and (2.91) is, from (2.63),

$$
\mathcal{L}_{6}=-T_{6}\left[\sqrt{-\operatorname{det}\left(e^{2 A-\phi / 6} \eta_{i j}+e^{2 B-\phi / 6} \partial_{i} Y^{m}(l) \partial_{j} Y_{m}(l)\right)}-e^{C}\right],
$$

corresponding to a potential

$$
V=T_{6}\left(e^{6 A-\phi / 2}-e^{C}\right)
$$

But this vanishes by the supersymmetry conditions (2.17). On the other hand, if the $N$ fivebrane had the opposite orientation, and hence the opposite $\tilde{e}_{6}$, then the sign change in the Wess-Zumino term in (2.128) would result in a net attractive force and therefore the corresponding configurations cannot be stable.

\subsection{The solitonic string}

Finally, just as the fivebrane was seen as a solitonic solution of string theory, one can re-interpret the elementary string solution of section 2.1 as a soliton of the fivebrane theory, i.e. as a solution of the source-free equations resulting from $I_{10}$ (fivebrane). The solution will be characterized by a non-vanishing topological "magnetic" charge $g_{2}$

$$
\frac{1}{\sqrt{2} \kappa} K=\tilde{g}_{2} \varepsilon_{7} / \Omega_{7}
$$

The solution is given by

$$
\begin{aligned}
e^{-2 \phi} & =e^{-2 \phi_{0}}\left(1+\frac{k_{2}}{y^{6}}\right) \\
d s^{2} & =e^{3\left(\phi-\phi_{0}\right) / 2} \eta_{\mu \nu} d x^{\mu} d x^{\nu}+e^{-\left(\phi-\phi_{0}\right) / 2} \delta_{m n} d x^{m} d x^{n}, \\
K & =6 k_{2} e^{-3 \phi_{0} / 2} \varepsilon_{7},
\end{aligned}
$$

where $\mu, \nu=0,1, m, n=2,3, \ldots, 9$ and where

$$
k_{2}=\frac{\kappa \tilde{g}_{2}}{3 \sqrt{2} \Omega_{7}} e^{3 \phi_{0} / 2} .
$$

It follows that the mass per unit length now saturates a bound involving the magnetic charge

$$
\mathcal{M}_{2}=\frac{1}{\sqrt{2}}\left|\tilde{g}_{2}\right| e^{\phi_{0} / 2}
$$


Note that the $\phi_{0}$ dependence is such that $\mathcal{M}_{2}$ is large for small $\mathcal{M}_{6}$ and vice-versa.

The electric charge of the elementary fivebrane and the magnetic charge of the solitonic string obey a Dirac quantization rule $[27,28]$

$$
\tilde{e}_{6} \tilde{g}_{2}=2 \pi n, \quad n=\text { integer },
$$

and hence from $(2.126)$

$$
\tilde{g}_{2}=2 \pi n / \sqrt{2} \kappa T_{6}
$$

The introduction of the elementary fivebrane and the solitonic string allows us to translate the Dirac quantization rules into a condition on the tensions of the string and fivebrane. From (2.55), (2.42) and (2.124) it follows that

$$
e_{2}=\tilde{g}_{2}, \quad \tilde{e}_{6}=g_{6}
$$

It then follows from $(2.44),(2.126),(2.53)$ and (2.134) that

$$
\kappa^{2} T_{2} T_{6}=|n| \pi, \quad n=\text { integer }
$$

\subsection{Singular or non-singular?}

Neither the elementary string nor the elementary fivebrane qualify for the epithet "soliton". They were obtained by coupling supergravity to the corresponding $\sigma$-models and hence display a $\delta$-function singularity at the location of the source $y=0$. Moreover, in both cases the curvature calculated from the $\sigma$-model metric blows up at $y=0$. To see this we rewrite the string solution (2.2) in the string $\sigma$-model metric (in what follows we set $\phi_{0}=0$ for simplicity)

$$
d s^{2}=\left(1+\frac{k_{2}}{y^{6}}\right)^{-1} \eta_{\mu \nu} d x^{\mu} d x^{\nu}+\delta_{m n} d x^{m} d x^{n} .
$$

We may verify that this metric exhibits a curvature singularity by computing the scalar curvature

$$
R_{\text {string }}(\text { string } \sigma-\text { model }) \sim-y^{-2} \text {. }
$$

Similarly, we rewrite the fivebrane solution (2.90) in the fivebrane $\sigma$-model metric

$$
d s^{2}=\left(1+\frac{k_{2}}{y^{2}}\right)^{-1 / 3} \eta_{\mu \nu} d x^{\mu} d x^{\nu}+\left(1+\frac{k_{2}}{y^{2}}\right)^{2 / 3} \delta_{m n} d x^{m} d x^{n}
$$


We may also verify that this metric exhibits a curvature singularity by computing the scalar curvature

$$
R_{\text {fivebrane }}(\text { fivebrane } \sigma-\text { model }) \sim-y^{-2 / 3} \text {. }
$$

(Incidentally, $R_{\text {string }}$ and $R_{\text {fivebrane }}$ both blow up as $y^{-1 / 2}$ in canonical variables.)

To justify that the solitonic string and fivebrane are worthy of their name, therefore, we must demonstrate that they are nonsingular. To show this, we now rewrite the string metric in fivebrane variables

$$
d s^{2}=\left(1+\frac{k_{2}}{y^{6}}\right)^{-2 / 3} \eta_{\mu \nu} d x^{\mu} d x^{\nu}+\left(1+\frac{k_{2}}{y^{6}}\right)^{1 / 3}\left(d y^{2}+y^{2} d \Omega_{7}^{2}\right)
$$

and the fivebrane metric in string variables

$$
d s^{2}=\eta_{\mu \nu} d x^{\mu} d x^{\nu}+\left(1+\frac{k_{6}}{y^{2}}\right)\left(d y^{2}+y^{2} d \Omega_{3}^{2}\right)
$$

Remarkably, both are free of curvature singularities, as may be seen by noting that, as $y \rightarrow 0$, the radius of $S^{7}$ in $(2.142)$ tends to the finite value $k_{2}^{1 / 6}$ and the radius of $S^{3}$ in $(2.143)$ tends to the finite value $k_{6}^{1 / 2}$. This is confirmed by a calculation of the scalar curvatures. We find

$$
\begin{gathered}
R_{\text {string }}(\text { fivebrane } \sigma-\text { model }) \sim+k_{2}^{-1 / 3} \\
R_{\text {fivebrane }}(\text { string } \sigma-\text { model }) \sim+k_{6}^{-1} .
\end{gathered}
$$

Note that throughout this report we have employed the fivebrane $\sigma$-model metric of $[29,26]$ given in (2.61), for which $g_{M N}($ fivebrane $\sigma-$ model $)=e^{-2 \phi / 3} g_{M N}($ string $\sigma-\operatorname{model})$. Now any metric $e^{-c \phi} g_{M N}$ (string $\sigma-$ model) will yield string solutions with non-singular curvature and any metric $e^{+c \phi} g_{M N}$ (fivebrane $\sigma$ - model) will yield fivebrane solutions with non-singular curvature provided $c \geq 2 / 3$. Interestingly enough, however, only the unique choice $c=2 / 3$ yields metrics which are also free of conical singularities [45,46]. From the point of view of metric singularities, therefore, the string is a singular solution of string theory and a nonsingular solution of fivebrane theory, whereas the fivebrane is a singular solution of fivebrane theory and a nonsingular solution of string theory. Thus the singularity structure is entirely symmetric between strings and fivebranes, in accordance with string/fivebrane duality.

We shall now show how to reach the same conclusion from a somewhat more physical test-probe/source approach [34]. Does a test-probe fall into the source in a finite proper 
time, as measured by its own clock, in which case the singularity is real, or in an infinite proper time, in which case the singularity is harmless? We find that the answer is probedependent. We shall show that when test probe and source are both strings or both fivebranes the singularity is real. By contrast, we shall show that if one is a string and the other a fivebrane, the singularity is harmless.

Let us consider the trajectory of a test string falling radially into a source string, oriented along $x^{1}=\xi^{1}$. For simplicity, let the test string lie either parallel or antiparallel to the source string. If we eliminate $\gamma_{i j}$ from (2.21) and substitute the solution of section 2.1 , we find that the lagrangian governing the dynamics of the test string is given by

$$
\mathcal{L}_{2}=-T_{2} e^{2 \phi}\left(\sqrt{\dot{t}^{2}-\dot{y}^{2} e^{-2 \phi}} \mp \dot{t}\right)
$$

where the minus (plus) sign corresponds to the parallel (antiparallel) configuration. The time derivative is with respect to $\xi^{0}$, which we choose to be the proper time $\tau$ measured by a clock at rest in the frame of the test string. From section 2.1 this is given by

$$
d \tau^{2}=-e^{\phi / 2} d s^{2}=e^{2 \phi} d t^{2}-d y^{2}
$$

Thus the calculation has been reduced to a one-dimensional problem and the dynamics of (2.146) is similar to that of a point particle whose mass is equal or opposite to its electric charge. Since there is no explicit time-dependence in $\mathcal{L}_{2}$, we have the following constant of the motion

$$
\frac{\partial \mathcal{L}_{2}}{\partial \dot{t}}=-T_{2} e^{2 \phi}\left(\frac{\dot{t}}{\sqrt{\dot{t}^{2}-\dot{y}^{2} e^{-2 \phi}}} \mp 1\right)=-T_{2} E .
$$

$E$ is the constant energy per unit mass of the motion and is determined from the intial conditions. Note that for the parallel strings case, we recover the zero static force result by noting that if $\dot{y}=0$ initially, then $E=0$ and $\dot{y}=0$ everywhere. We also recover the vanishing leading order (in the velocity) dynamic force result found in [20]. From (2.148) we obtain an expression for the coordinate velocity

$$
\left(\frac{d y}{d t}\right)^{2}=\frac{E^{2} e^{-2 \phi} \pm 2 E}{\left(E e^{-2 \phi} \pm 1\right)^{2}}
$$

We now wish to relate the radial position to the proper time. Combining (2.149) and (2.147) we obtain

$$
\left(\frac{d y}{d \tau}\right)^{2}=e^{-2 \phi}\left(E^{2} e^{-2 \phi} \pm 2 E\right)
$$


for the proper velocity in terms of the radial position. The acceleration can be obtained by differentiating (2.150) with respect to $\tau$ and replacing (2.150) in the resulting expression. The acceleration written in terms of the position is independent of the sign of the velocity and is given by

$$
\frac{d^{2} y}{d \tau^{2}}=-\frac{6 k_{2} E^{2}}{y^{7}}\left(1+\frac{k_{2}}{y^{6}} \pm E^{-1}\right) .
$$

For parallel strings, the force is always attractive when initially $\dot{y} \neq 0$. For antiparallel strings, the acceleration is always inward, and the test string does indeed fall towards the source string. We may thus choose the negative sign for the square roots in (2.149) and (2.150). To calculate the proper time taken for the test string to reach the source string, we rewrite (2.150) and integrate

$$
\tau_{0}=\int_{0}^{\tau_{0}} d \tau=\int_{0}^{y_{0}} \frac{d y}{\sqrt{e^{-2 \phi}\left(E^{2} e^{-2 \phi} \pm 2 E\right)}} .
$$

On using the expression for $\phi$ from section 2.1, we note that $\tau_{0}$ is finite. Thus the test string falls into the source string in a finite amount of time, and the singularity is real. In particular, let us focus on the case where the test string is antiparallel to the source string. If $\dot{y}=0$ at $y=y_{0}$, then

$$
E=2 e^{2 \phi\left(y_{0}\right)}=\frac{2}{1+k_{2} / y_{0}^{6}} .
$$

Let $x \equiv y / y_{0}$, then $\tau_{0}$ can be written as

$$
\tau_{0}=\frac{e^{-2 \phi\left(y_{0}\right)} y_{0}^{4}}{2 \sqrt{k_{2}}} \int_{0}^{1} \frac{d x x^{6}}{\sqrt{\left(x^{6}+k_{2} / y_{0}^{6}\right)\left(1-x^{6}\right)}}
$$

For large $y_{0}$, we find that $\tau_{0} \sim k_{2}^{-1 / 2}$. Since the mass per unit length of the string is given by $\mathcal{M}_{2}=T_{2}$ [19], this means that $\tau_{0} \sim \mathcal{M}_{2}^{-1 / 2}$, which is the same dependence of the time on the mass for an observer falling into a Schwarzschild black hole. Just as for the black hole case, moreover, it is easy to see from (2.150) and (2.151) that the proper velocity and acceleration both tend to infinity as the test string approaches the singularity. To further strengthen the analogy with a black hole-type singularity, one can calculate the elapsed distant observer time for the fall. In this case one can easily show that $t_{0} \rightarrow \infty, d y / d t \rightarrow 0$ and $d^{2} y / d t^{2} \rightarrow 0$ as the test string approaches the singularity. In other words, the distant observer never sees the test string reach the singularity. In this case, the event horizon is at the singularity. 
We shall now repeat the above calculation for a test-fivebrane falling radially into a source fivebrane oriented along $x^{a}=\xi^{a},(a=1, \ldots, 5)$. Again, we let the test fivebrane lie either parallel or antiparallel to the source fivebrane, i.e. with the same or opposite orientation. If we eliminate $\gamma_{i j}$ from (2.63) and the fivebrane solution of section 2.4, we find that the lagrangian governing the dynamics of the test fivebrane is given by

$$
\mathcal{L}_{6}=-T_{6} e^{-2 \phi}\left(\sqrt{\dot{t}^{2}-\dot{y}^{2} e^{2 \phi}} \mp \dot{t}\right)
$$

where the minus (plus) sign corresponds to the parallel (antiparallel) configuration. The time derivative is with respect to $\xi^{0}$, which we choose to be the proper time $\tau$ measured by a clock at rest in the frame of the test fivebrane. From section 2.4 this is given by

$$
d \tau^{2}=-e^{-\phi / 6} d s^{2}=e^{-2 \phi / 3}\left(d t^{2}-e^{2 \phi} d y^{2}\right) .
$$

This time the Euler-Lagrange equations yield the following constant of the motion

$$
\frac{\partial \mathcal{L}_{6}}{\partial \dot{t}}=-T_{6} e^{-2 \phi}\left(\frac{\dot{t}}{\sqrt{\dot{t}^{2}-\dot{y}^{2} e^{2 \phi}}} \mp 1\right)=-T_{6} E
$$

From (2.157) we obtain an expression for the coordinate velocity

$$
\left(\frac{d y}{d t}\right)^{2}=\frac{E^{2} e^{2 \phi} \pm 2 E}{\left(E e^{2 \phi} \pm 1\right)^{2}}
$$

Combining (2.158) and (2.90) we obtain

$$
\left(\frac{d y}{d \tau}\right)^{2}=e^{2 \phi / 3}\left(E^{2} e^{2 \phi} \pm 2 E\right)
$$

for the proper velocity in terms of the radial position. The acceleration can be obtained by differentiating (2.159) with respect to $\tau$ and replacing (2.159) in the resulting expression. The acceleration written in terms of the position is independent of the sign of the velocity as in the string-string case and is again attractive, so the test fivebrane does indeed fall towards the source fivebrane. To calculate the proper time taken for the test fivebrane to reach the source fivebrane we rewrite (2.159) and integrate

$$
\tau_{0}=\int_{0}^{\tau_{0}} d \tau=\int_{0}^{y_{0}} \frac{d y}{\sqrt{e^{2 \phi / 3}\left(E^{2} e^{2 \phi} \pm 2 E\right)}} .
$$


On using the expression for $\phi$ from section 2.4, we note again that $\tau_{0}$ is manifestly finite. Thus the test fivebrane falls into the source fivebrane in a finite amount of time, and the singularity is real. In the antiparallel case, the dependence of the time on the mass of a source for the test fivebrane initially at rest and for large initial separation is again of the form $\tau_{0} \sim k_{6}^{-1 / 2}$. Since the mass per unit 5 -volume of the fivebrane is given by $\mathcal{M}_{6}=T_{6}$ [29], this means $\tau_{0} \sim \mathcal{M}_{6}^{-1 / 2}$ as for the string. It is easy to see that the proper velocity and acceleration both $\rightarrow \infty$ as the test fivebrane approaches the singularity and that $t_{0} \rightarrow \infty$, $d y / d t \rightarrow 0$ and $d^{2} y / d t^{2} \rightarrow 0$. Once more, the event horizon is located at the singularity.

An entirely different state of affairs holds for a test string moving in the background of a source fivebrane or, by duality, a test fivebrane moving in the background of a source string. In this case, the test probe takes an infinite amount of proper time to reach the source. First we consider the trajectory of a test string falling radially into a source fivebrane, oriented along $x^{a}=\xi^{a}(a=1,2, \ldots, 5)$. Let the test string lie either parallel or antiparallel to one of the fivebrane directions, say $x^{1}$. From section 2.4 , the only nonvanishing components of $K$ are of the form $K_{012345 m}$, where the directions $m=6,7,8,9$ are transverse to the fivebrane. By dualizing, we see that the only nonzero components of $H=d B$ are $H_{p q r}(y)$, where again, $p, q, r=6,7,8,9$. It follows that the only nonzero components of $B_{M N}$ occur when $M, N=6,7,8,9$. It then follows that the WZW term $\epsilon^{i j} \partial_{i} X^{M} \partial_{j} X^{N} B_{M N}$ vanishes. Substituting the fivebrane solution in (2.21), we find that the test string lagrangian reduces to

$$
\mathcal{L}_{2}=-T_{2} \sqrt{\dot{t}^{2}-e^{2 \phi} \dot{y}^{2}}
$$

for purely radial motion. From (2.50) and (2.20), the proper time is given by

$$
d \tau^{2}=-e^{\phi / 2} d s^{2}=d t^{2}-e^{2 \phi} d y^{2}
$$

Again we have a constant of the motion

$$
\frac{\partial \mathcal{L}_{2}}{\partial \dot{t}}=-T_{2} \frac{\dot{t}}{\sqrt{\dot{t}^{2}-e^{2 \phi} \dot{y}^{2}}}=-T_{2} E
$$

Note that $E=1$ corresponds to a zero static force. We invert (2.163) to obtain the coordinate velocity

$$
\left(\frac{d y}{d t}\right)^{2}=e^{-2 \phi}\left(1-1 / E^{2}\right)
$$


Combining (2.162) and (2.163), we obtain the proper velocity

$$
\left(\frac{d y}{d \tau}\right)^{2}=\left(E^{2}-1\right) e^{-2 \phi}
$$

The acceleration is given by

$$
\frac{d^{2} y}{d \tau^{2}}=\frac{k_{6}\left(E^{2}-1\right) e^{-4 \phi}}{y^{3}} .
$$

Note that the acceleration is repulsive in this case. In both the $y \rightarrow 0$ and $y \rightarrow \infty$ limits, the acceleration vanishes (an asymptotic freedom of some sort). We assume that the string is directed towards the fivebrane initially. The time taken for the fall from an initial position $y_{0}$

$$
\tau_{0}=\frac{1}{\sqrt{E^{2}-1}} \int_{0}^{y_{0}} e^{\phi} d y
$$

diverges logarithmically with $y$. Therefore it takes the string an infinite amount of proper time to reach the singularity. In other words, the string never sees the singularity, and as far as the string is concerned, the singularity is not real.

An analogous calculation for a test fivebrane falling towards a source string shows that the string is nonsingular as a source for fivebranes. For a test fivebrane with one of its spatial directions parallel to the string, the WZW term again vanishes, as in the above case. In this case, substituting the string solution into (2.63), the lagrangian reduces to

$$
\mathcal{L}_{6}=-T_{6} \sqrt{\dot{t}^{2}-e^{-2 \phi} \dot{y}^{2}}
$$

for purely radial motion. From the $(2.131)$ and $(2.61)$, the proper time is given by

$$
d \tau^{2}=-e^{-\phi / 6} d s^{2}=e^{4 \phi / 3}\left(d t^{2}-e^{-2 \phi} d y^{2}\right) .
$$

Again we have a constant of the motion

$$
\frac{\partial \mathcal{L}_{6}}{\partial \dot{t}}=-T_{6} \frac{\dot{t}}{\sqrt{\dot{t}^{2}-e^{2 \phi} \dot{y}^{2}}}=-T_{6} E .
$$

Again $E=1$ corresponds to a zero static force. We invert (2.170) to obtain the coordinate velocity

$$
\left(\frac{d y}{d t}\right)^{2}=e^{2 \phi}\left(1-1 / E^{2}\right) .
$$

Combining (2.171) and (2.169) we obtain the proper velocity

$$
\left(\frac{d y}{d \tau}\right)^{2}=\left(E^{2}-1\right) e^{2 \phi / 3}
$$


The acceleration is again found to be repulsive and is given by

$$
\frac{d^{2} y}{d \tau^{2}}=\frac{k_{2}\left(E^{2}-1\right) e^{8 \phi / 3}}{y^{7}} .
$$

Again the acceleration vanishes in both the $y \rightarrow 0$ and $y \rightarrow \infty$ limits. Now assume that the fivebrane is directed towards the string initially. The time taken for the fall from an initial position $y_{0}$ is given by

$$
\tau_{0}=\frac{1}{\sqrt{E^{2}-1}} \int_{0}^{y_{0}} e^{-\phi / 3} d r
$$

and again diverges logarithmically with $y$. Therefore it takes the fivebrane an infinite amount of proper time to reach the singularity. In other words, the fivebrane never sees the singularity, and as far as the fivebrane is concerned, the string singularity is not real.

We have seen that, as far as singularities are concerned, the superstring and the superfivebrane solitons are on an equal footing: the fivebrane is a singular solution of fivebrane theory but a non-singular solution of string theory while the string is a singular solution of string theory but a non-singular solution of fivebrane theory. What is asymmetric, however, is the state of current technology. One can prove rigorously that $I_{10}$ (string) describes the field theory limit of string theory and that the string loop coupling constant is, from (2.20) given by $g$ (string) $=e^{\phi_{0}}$; one has only plausibility arguments that the dual action $I_{10}$ (fivebrane) describes the field-theory limit of fivebrane theory and that the fivebrane loop coupling constant is, from (2.61), $g$ (fivebrane) $=e^{-\phi_{0} / 3}$ and hence that the strong coupling limit of the string corresponds to the weakly coupled fivebrane and vice-versa $[26]$.

\subsection{Strings and fivebranes as interpolating solitons}

In this section we discuss how in string $\sigma$-model metric, the solitonic fivebrane solution of $D=10$ supergravity interpolates [45] between $D=10$ Minkowski spacetime and a supersymmetric $S^{3}$ compactification to a linear dilaton vacuum [47]. Furthermore, in fivebrane sigma-model metric, the solitonic string solution of $D=10$ supergravity interpolates [46] between $D=10$ Minkowski spacetime and a supersymmetric $S^{7}$ compactification to a three-dimensional anti-de Sitter generalization of the linear dilaton vacuum, which may be invariantly characterized in terms of conformal Killing vectors [47]. 
Let us first consider the solitonic fivebrane solution of section 2.4 in string variables rewritten in terms of a new radial coordinate $r^{2}=y^{2}+b^{2}$ where $b^{2}=k_{6}$

$$
\begin{aligned}
d s^{2} & =-d t^{2}+d \mathbf{x} \cdot d \mathbf{x}+\left(1-\frac{b^{2}}{r^{2}}\right)^{-2} d r^{2}+r^{2} d \Omega_{3}^{2} \\
e^{-2 \phi} & =1-\frac{b^{2}}{r^{2}} \\
H & =2 b^{2} \varepsilon_{3},
\end{aligned}
$$

where $d \mathbf{x} \cdot d \mathbf{x}$ is the Euclidean 5-metric, $d \Omega_{3}^{2}$ the standard metric on the unit 3-sphere and $\varepsilon_{3}$ its volume 3 -form. To determine the asymptotic form of the metric near $r=b$ we let

$$
r=b\left(1+\frac{e^{2 \rho / b}}{2}\right) .
$$

In the limit $r=b(2.175)$ reduces to

$$
\begin{aligned}
d s^{2} & \sim\left(-d t^{2}+d \mathbf{x} \cdot d \mathbf{x}+d \rho^{2}\right)+b^{2} d \Omega_{3}^{2} \\
\phi & \sim-\frac{1}{b} \rho \\
H & \sim 2 b^{2} \varepsilon_{3}
\end{aligned}
$$

which is $M_{7} \times S^{3}$, with a linear dilaton vacuum. That is, in string sigma-model metric the solitonic fivebrane interpolates between ten-dimensional Minkowski spacetime and the product of $S^{3}$ with a seven-dimensional Minkowski spacetime.

Next we consider the solitonic string solution of section 2.5 in fivebrane variables written in terms of a new radial coordinate $r^{6}=y^{6}+b^{6}$ where $b^{6}=k_{2}$

$$
\begin{aligned}
d s^{2} & =\left(1-\frac{b^{6}}{r^{6}}\right)^{\frac{2}{3}}\left(-d t^{2}+d \sigma^{2}\right)+\left(1-\frac{b^{6}}{r^{6}}\right)^{-2} d r^{2}+r^{2} d \Omega_{7}^{2} \\
e^{-2 \phi} & =\left(1-\frac{b^{6}}{r^{6}}\right)^{-1} \\
K & =6 b^{6} \varepsilon_{7} .
\end{aligned}
$$

This time we define

$$
r=a\left(1+\frac{e^{6 \rho / b}}{6}\right) .
$$

Near $r=b(2.178)$ reduces to

$$
\begin{aligned}
d s^{2} & \sim e^{4 \rho / b}\left(-d t^{2}+d \sigma^{2}\right)+d \rho^{2}+b^{2} d \Omega_{7}^{2} \\
\phi & \sim \frac{3}{b} \rho \\
K & \sim 6 b^{6} \varepsilon_{7} .
\end{aligned}
$$


The metric is the standard metric on the product of $S_{7}$ with three-dimensional anti-de Sitter space, $a d S_{3}$. Clearly, the dilaton is linear only for the special choice of coordinates used here, and since the spacetime factor of the asymptotic metric is not Minkowski (as it was for the fivebrane solution) but rather $a d S_{3}$, the geometrical significance of linearity is unclear from the above result. We verify below that the $S^{7}$ compactification of $\mathrm{D}=10$ supergravity implied by this analysis indeed exists, incidentally elucidating the geometrical significance of 'linear' in the adS context.

Let $\left\{x^{M} ; M=0,1, \ldots, 9\right\}$ be coordinates for the ten-dimensional spacetime, and define

$$
\Phi=e^{2 \phi / 3}
$$

The field equations of (2.87) can now be written as

$$
\begin{gathered}
R_{M N}=\Phi^{-1} D_{M} D_{N} \Phi+\frac{1}{2.6 !} K_{M P_{1} \ldots P_{6}} K_{N} P_{1} \ldots P_{6}-\frac{1}{3.7 !} g_{M N} K^{2} \\
\partial_{M}\left(\sqrt{-g} \Phi K^{M N_{1} \ldots N_{6}}\right)=0 \\
\square \Phi=\frac{1}{3.7 !} K^{2} \Phi .
\end{gathered}
$$

We now split the coordinates $\left\{x^{M}\right\}$ into $\left\{x^{\mu}, y^{m}\right\}$ with $\mu=0,1,2$ and $m=1, \ldots, 7$, and seek product metrics of the form

$$
g_{\mu \nu}=g_{\mu \nu}(x) \quad g_{m n}=g_{m n}(y)
$$

with $g_{\mu n}=0$. In this case, $\sqrt{-g}=e_{3}(x) e_{7}(y)$ where $e_{3}$ and $e_{7}$ are the scalar density volume factors for the three and seven-dimensional spaces, respectively. It follows that (2.183) is now solved by setting

$$
K^{m_{1} \ldots m_{7}}=3 m\left(e_{7}\right)^{-1} \varepsilon^{m_{1} \ldots m_{7}}
$$

for some constant $m$, with all other components of $K$ vanishing, whereupon (2.184) becomes

$$
\left(\square-3 m^{2}\right) \Phi=0
$$

We now suppose that $\Phi=\Phi(x)$, and further that

$$
D_{\mu} \partial_{\nu} \Phi=\frac{1}{3} g_{\mu \nu} \square \Phi
$$


or, equivalently, in view of (2.187),

$$
D_{\mu} \partial_{\nu} \Phi=m^{2} g_{\mu \nu} \Phi
$$

which implies (2.187) and therefore supercedes it. Given (2.189), (2.182) reduces to the two equations

$$
R_{\mu \nu}=-2 m^{2} g_{\mu \nu} \quad R_{m n}=\frac{3}{2} m^{2} g_{m n}
$$

with $R_{m \nu}=0$. These equations are solved by the standard invariant metrics on $a d S_{3}$ and $S^{7}$ respectively. It remains to solve $(2.189)$. We can choose coordinates $(t, \sigma, \rho)$ on $a d S_{3}$ such that the $a d S_{3}$ metric is

$$
d s^{2}=e^{2 m \rho}\left(-d t^{2}+d \sigma^{2}\right)+d \rho^{2}
$$

In these coordinates it is straightforward to verify that

$$
\phi=\frac{3 m}{2} \rho
$$

solves (2.189). We have therefore found an $S^{7}$ compactification of $D=10$ supergravity to $a d S_{3}$ with a linear dilaton. Setting $m=2 / b$ we recover $(2.180)$, found previously as an asymptotic limit of the extreme string solution.

Observe now that (2.188) implies that $g^{\mu \nu} \partial_{\mu} \Phi \partial_{\nu}$ is a conformal Killing vector of $a d S_{3}$. We have shown above that an eigenfunction of the Dalembertian on $a d S_{3}$ with eigenvalue $3 m^{2}$ is the potential for a conformal Killing vector of $a d S_{3}$. A similar observation was made previously in the context of an $S^{3}$ compactification of $D=10$ supergravity [47] to $a d S_{7}$. In fact, both the $S^{3}$ and $S^{7}$ compactifications exhibited above are obtained from the solutions found in [47] by the analytic continuation $m \rightarrow i m$. We now see that the linear dilaton can be characterized in a coordinate-free way as proportional to the logarithm of a conformal Killing potential. 


\section{3. (d-1)-branes in diverse dimensions}

\subsection{Diverse dimensions}

In the previous section, we focused on spacetime dimension $D=10$, worldvolume dimension $d=2$ and dual worldvolume dimension $\tilde{d}=8-2=6$. The purpose of the present section is to see how far these results generalize to arbitrary $D, d$, and $\tilde{d}$. We present a generic Lagrangian, in arbitrary spacetime dimension $D$, describing the interaction of a dilaton, a graviton and an antisymmetric tensor of arbitrary rank $d$. For each $D$ and $d$, we find black $\tilde{p}$-brane solutions where $\tilde{p}=\tilde{d}-1$ and $\tilde{d}=D-d-2$. These solutions display a spacetime singularity surrounded by an event horizon, and are characterized by a mass per unit $\tilde{p}$-volume $\mathcal{M}_{\tilde{d}}$, and topological "magnetic" charge $g_{\tilde{d}}$, obeying $\sqrt{2} \kappa \mathcal{M}_{\tilde{d}} \geq g_{\tilde{d}}$. The theory also admits elementary $p$-brane solutions with "electric" Noether charge $e_{d}$, obeying the Dirac quantization rule $e_{d} g_{\tilde{d}}=2 \pi n, n=$ integer. We then present the Lagrangian describing the theory dual to the original theory, whose antisymmetric tensor has rank $\tilde{d}$ and for which the roles of topological and elementary solutions are interchanged. In the extreme limits $\sqrt{2} \kappa \mathcal{M}_{\tilde{d}}=g_{\tilde{d}}$ or $\sqrt{2} \kappa \mathcal{M}_{d}=e_{d}$, the singularity and event horizon coalesce. As discussed in section 4 , for specific values of $D$ and $d$, these extreme solutions also exhibit supersymmetry and may be identified with previously classified heterotic, Type IIA and Type IIB super $\tilde{p}$-branes. Curiously enough, the results obtained in section 2 on the singularity structure of strings and fivebranes do not generalize to arbitrary $d$.

In section 3.2, we write down a general action in $D$ spacetime dimensions describing the interaction of an antisymmetric tensor potential of rank $d$ with gravity and a dilaton. We allow these fields to couple to an elementary $d$-dimensional extended object, (a $p$ brane, with $d=p+1$ ) and define an "electric" Noether charge associated with it. This construction involves, in particular, the identification of the $p$-brane $\sigma$-model metric in terms of the dilaton and the canonical metric.

In section 3.3, we show how the combined field equations admit solutions describing such elementary objects, in much the same way as the elementary string emerged as a solution of supergravity coupled to a string $\sigma$-model source in section 2.1. The mass per unit $p$-volume $\mathcal{M}_{d}$ and the Noether charge $e_{d}$ of these elementary solutions satisfy the 
equality $\sqrt{2} \kappa \mathcal{M}_{d}=\left|e_{d}\right|$, which is the same relation found in section 2 for the supersymmetric string and fivebrane. One may also demonstrate a no-force condition by showing that the mutual gravitational-dilaton attraction of two such $p$-branes of the same orientation is exactly cancelled by an equal and opposite contribution from the antisymmetric tensor. This permits the construction of stable multi-p-brane solutions.

In addition to the singular elementary $(d-1)$-brane solutions carrying non-zero "electric" Noether charge $e_{d}$, the theory also admits non-singular soliton $(\tilde{d}-1)$-brane solutions, where $\tilde{d}=D-d-2$. As described in section 3.4, these solutions are dual to the elementary solutions and carry a non-zero "magnetic" topological charge $g_{\tilde{d}}$, obeying the Dirac quantization rule $[27,28]$

$$
e_{d} g_{\tilde{d}}=2 \pi n, \quad n=\text { integer. }
$$

In section 3.5 we consider the theory dual to the theory of section 3.2 , for which the roles of antisymmetric tensor field equations and Bianchi identities, and hence electric and magnetic charges, are interchanged. This, together with our result for the $\sigma$-model metric of section 3.2 , leads to a relation between the loop expansion parameter $\mathrm{g}_{d}$ of the $(d-1)$-brane and the loop expansion parameter $\mathrm{g}_{\tilde{d}}$ of the $(\tilde{d}-1)$-brane. We find

$$
\mathrm{g}_{d}^{d}=1 / \mathrm{g}_{\tilde{d}}^{\tilde{d}}
$$

thus confirming that strongly coupled $(d-1)$ branes correspond to weakly coupled $(\tilde{d}-1)$ branes and vice versa. The introduction of the $\sigma$-model metric permits a useful comparison with Brans-Dicke theory.

$D=6$ is of special interest because in this dimension a string is dual to another string. Moreover, as discussed in section 3.6, this permits the construction of a "dyonic" [48] solution carrying both electric charge $e_{2}$ and magnetic charge $g_{2}$. In the limit $e_{2}=g_{2}$, this solution reduces to the previously derived self-dual superstring [49].

Although many of the results of section 2 generalize in an obvious way to arbitrary $d$ and $D$, this is not the case for the issue of singularities. We show in section 3.7 that although the curvature of the $(\tilde{d}-1)$-brane metric written in the dual $(d-1)$-brane $\sigma$ model variables is still finite at $y=0$ for all $d$ and $D$, the proper time for an infalling test $(d-1)$-brane to reach a source $(\tilde{d}-1)$-brane is finite only for particles and strings and their duals.

Black $p$-branes, solutions with $\mathcal{M}_{\tilde{d}} \geq \frac{1}{\sqrt{2}} g_{\tilde{d}}$ are discussed in section 3.8. These solutions exhibit singularities shielded by an event horizon. As special cases, we recover the $D=10$ black $p$-branes $(p=0, \ldots 6)$ of [50], the $D=11$ black $p$-branes $(p=2,5)$ of $[51]$ and the $D=4$ Kaluza-Klein black hole $(p=1)[52,53,54,55]$. 


\subsection{The action}

Consider an antisymmetric tensor potential of rank $d, A_{M_{1} M_{2} \ldots M_{d}}$, in $D$ spacetime dimensions $(M=0,1, \ldots(D-1))$ interacting with gravity, $g_{M N}$, and the dilaton, $\phi$, via the action

$$
I_{D}(d)=\frac{1}{2 \kappa^{2}} \int d^{D} x \sqrt{-g}\left(R-\frac{1}{2}(\partial \phi)^{2}-\frac{1}{2(d+1) !} e^{-a(d) \phi} F_{d+1}^{2}\right)
$$

where the rank $(d+1)$ field strength $F_{d+1}$ is given by

$$
F_{d+1}=d A_{d}
$$

and $a(d)$ is an, as yet undetermined, constant. Special cases of this action have been considered before in the context of classical solutions [56]. Here we keep both $D$ and $d$ arbitrary. We allow these fields to couple to an elementary $d$-dimensional extended object (a "( $d-1)$-brane") whose trajectory is given by $X^{M}\left(\xi^{i}\right)(i=0,1, \ldots(d-1))$, worldvolume metric by $\gamma_{i j}(\xi)$, and tension by $T_{d}$, via the action

$$
\begin{aligned}
S_{d}=T_{d} \int d^{d} \xi( & -\frac{1}{2} \sqrt{-\gamma} \gamma^{i j} \partial_{i} X^{M} \partial_{j} X^{N} g_{M N} e^{a(d) \phi / d}+\frac{(d-2)}{2} \sqrt{-\gamma} \\
& \left.-\frac{1}{d !} \varepsilon^{i_{1} i_{2} \ldots i_{d}} \partial_{i_{1}} X^{M_{1}} \partial_{i_{2}} X^{M_{2}} \ldots \partial_{i_{d}} X^{M_{d}} A_{M_{1} M_{2} \ldots M_{d}}\right) .
\end{aligned}
$$

The $\phi$ dependence is chosen so that under the rescaling

$$
\begin{aligned}
g_{M N} & \rightarrow \lambda^{2 d /(D-2)} g_{M N}, \\
A_{M_{1} M_{2} \ldots M_{d}} & \rightarrow \lambda^{d} A_{M_{1} M_{2} \ldots M_{d}}, \\
e^{\phi} & \rightarrow \lambda^{2 d(D-d-2) /(D-2) a(d)} e^{\phi}, \\
\gamma_{i j} & \rightarrow \lambda^{2} \gamma_{i j},
\end{aligned}
$$

both actions scale the same way

$$
\begin{aligned}
I_{D}(d) & \rightarrow \lambda^{d} I_{D}(d), \\
S_{d} & \rightarrow \lambda^{d} S .
\end{aligned}
$$

The field equations and Bianchi identities of the $A$ field may be written

$$
d^{*}\left(e^{-a(d) \phi} F\right)=2 \kappa^{2}(-)^{d^{2} *} J,
$$




$$
d F \equiv 0
$$

where the rank $d$ source $J$ is given by

$$
J^{M_{1} \ldots M_{d}}=T_{d} \int d^{d} \xi \varepsilon^{i_{1} i_{2} \ldots i_{d}} \partial_{i_{1}} X^{M_{1}} \partial_{i_{2}} X^{M_{2}} \ldots \partial_{i_{d}} X^{M_{d}} \frac{\delta^{D}(x-X)}{\sqrt{-g}}
$$

Let us introduce the dual worldvolume dimension, $\tilde{d}$, by

$$
\tilde{d} \equiv D-d-2
$$

We may now define two conserved charges: the Noether "electric" charge

$$
e_{d}=\frac{1}{\sqrt{2} \kappa} \int_{S_{\tilde{d}+1}} e^{-a(d) \phi *} F
$$

where $S^{\tilde{d}+1}$ is the $(\tilde{d}+1)$-sphere surrounding the elementary $(d-1)$ brane, and the topological "magnetic" charge

$$
g_{\tilde{d}}=\frac{1}{\sqrt{2} \kappa} \int_{S^{d+1}} F .
$$

This latter charge will be non-zero if the action $I_{D}$ admits a solitonic $\tilde{d}$-dimensional extended object (a "( $\tilde{d}-1)$-brane"). These charges obey a Dirac quantization condition $[27,28]$

$$
\frac{e_{d} g_{\tilde{d}}}{4 \pi}=\frac{n}{2}, \quad n=\text { integer }
$$

analogous to the $(d=1, D=4)$ condition that relates electric and magnetic charges. At this stage, of course, it is not yet obvious that the system admits either elementary or solitonic extended object solutions, nor if they do, what are the values of the electric and magnetic charges $e_{d}$ and $g_{\tilde{d}}$.

Let us first consider the field equations resulting from $I_{D}+S_{d}$. The Einstein equation is

$$
\begin{aligned}
\sqrt{-g} & {\left[R^{M N}-\frac{1}{2} g^{M N} R-\frac{1}{2}\left(\partial^{M} \phi \partial^{N} \phi-\frac{1}{2} g^{M N}(\partial \phi)^{2}\right)\right.} \\
& \left.-\frac{1}{2} \frac{1}{d !}\left(F^{M}{ }_{M_{1} \ldots M_{d}} F^{N M_{1} \ldots M_{d}}-\frac{1}{2(d+1)} g^{M N} F^{2}\right) e^{-a(d) \phi}\right] \\
& =\kappa^{2} \sqrt{-g} T^{M N}((d-1)-\text { brane }),
\end{aligned}
$$


where the energy-momentum tensor is given by

$$
T^{M N}((d-1)-\text { brane })=-T_{d} \int d^{d} \xi \sqrt{-\gamma} \gamma^{i j} \partial_{i} X^{M} \partial_{j} X^{N} e^{a \phi / d} \frac{\delta^{D}(x-X)}{\sqrt{-g}},
$$

the antisymmetric tensor equation is

$$
\partial_{M}\left(\sqrt{-g} e^{-a \phi} F^{M M_{1} \ldots M_{d}}\right)=2 \kappa^{2} T_{d} \int d^{d} \xi \varepsilon^{i_{1} \ldots i_{d}} \partial_{i_{1}} X^{M_{1}} \ldots \partial_{i_{d}} X^{M_{d}} \delta^{D}(x-X)
$$

and the dilaton equation is

$$
\begin{aligned}
& \partial_{M}\left(\sqrt{-g} g^{M N} \partial_{N} \phi\right)+\frac{a(d)}{2(d+1) !} \sqrt{-g} e^{-a(d) \phi} F^{2} \\
& =\frac{a(d) \kappa^{2} T_{d}}{d} \int d^{d} \xi \sqrt{-\gamma} \gamma^{i j} \partial_{i} X^{M} \partial_{j} X^{N} g_{M N} e^{a(d) \phi / d} \delta^{D}(x-X) .
\end{aligned}
$$

Furthermore, the $(d-1)$-brane field equations are

$$
\begin{aligned}
\partial_{i}\left(\sqrt{-\gamma} \gamma^{i j} \partial_{j} X^{N} g_{M N} e^{a(d) \phi / d}\right) & -\frac{1}{2} \sqrt{-\gamma} \gamma^{i j} \partial_{i} X^{N} \partial_{j} X^{P} \partial_{M}\left(g_{N P} e^{a(d) \phi / d}\right) \\
& -\frac{1}{d !} \varepsilon^{i_{1} \ldots i_{d}} \partial_{i_{1}} X^{M_{1}} \ldots \partial_{i_{d}} X^{M_{d}} F_{M M_{1} \ldots M_{d}}=0
\end{aligned}
$$

and

$$
\gamma_{i j}=\partial_{i} X^{M} \partial_{j} X^{N} g_{M N} e^{a(d) \phi / d}
$$

\subsection{The elementary $(d-1)$-brane}

To solve these coupled field- $(d-1)$-brane equations we begin by making an ansatz for the $D$-dimensional metric $g_{M N}, d$-form $A_{M_{1}} \ldots M_{d}$, dilaton $\phi$ and coordinates $X^{M}(\xi)$ corresponding to the most general $d /(D-d)$ split invariant under $P_{d} \times S O(D-d)$ where $P_{d}$ is the $d$-dimensional Poincaré group. We split the indices

$$
x^{M}=\left(x^{\mu}, y^{m}\right)
$$

where $\mu=0,1 \ldots(d-1)$ and $m=d, d+1, \ldots(D-1)$, and write the line-element as

$$
d s^{2}=e^{2 A} \eta_{\mu \nu} d x^{\mu} d x^{\nu}+e^{2 B} \delta_{m n} d y^{m} d y^{n}
$$


and the $d$-form gauge field as

$$
A_{\mu_{1} \ldots \mu_{d}}=-\frac{1}{d g} \varepsilon_{\mu_{1} \ldots \mu_{d}} e^{C}
$$

where ${ }^{d} g$ is the determinant of $g_{\mu \nu}, \varepsilon_{\mu_{1} \ldots \mu_{d}} \equiv g_{\mu_{1} \nu_{1}} \ldots g_{\mu_{d} \nu_{d}} \varepsilon^{\nu_{1} \ldots \nu_{d}}$ and $\varepsilon^{012 \ldots(d-1)}=1$ i.e. $A_{01 \ldots(d-1)}=-e^{C}$. All other components of $A_{M_{1} \ldots M_{d}}$ are set to zero. $P_{d}$ invariance requires that the arbitrary functions $\mathrm{A}, \mathrm{B}, \mathrm{C}$ depend only on $y^{m} ; S O(D-d)$ invariance then requires that this dependence be only through $y=\sqrt{\delta_{m n} y^{m} y^{n}}$. Similarly our ansatz for the dilaton is

$$
\phi=\phi(y)
$$

In the $(d-1)$-brane sector we also split

$$
X^{M}=\left(X^{\mu}, Y^{m}\right)
$$

and make the static gauge choice

$$
X^{\mu}=\xi^{\mu}
$$

and the ansatz

$$
Y_{m}=\text { constant. }
$$

Substituting these ansatz into (3.20) yields

$$
\gamma_{i j}=e^{2 A+a(d) \phi / d} \eta_{i j}
$$

and the only non-vanishing components of the field strength are

$$
F_{m \mu_{1} \ldots \mu_{d}}=-\frac{1}{d g} \varepsilon_{\mu_{1} \ldots \mu_{d}} \partial_{m} e^{C}
$$

Then the $\mu \nu$ components of the Einstein equation (3.15) reduce to a single equation

$$
\begin{aligned}
& e^{(d-2) A+\tilde{d} B} \delta^{m n}\left[(d-1) \partial_{m} \partial_{n} A+\frac{d(d-1)}{2} \partial_{m} A \partial_{n} A+(\tilde{d}+1) \partial_{m} \partial_{n} B\right. \\
& +\frac{(\tilde{d}+1) \tilde{d}}{2} \partial_{m} B \partial_{n} B+\tilde{d}(d-1) \partial_{m} A \partial_{n} B \\
& \left.+\frac{1}{4} e^{-2 d A+2 C-a(d) \phi} \partial_{m} C \partial_{n} C+\frac{1}{4} \partial_{m} \phi \partial_{n} \phi\right] \\
& =-\kappa^{2} T_{d} e^{(d-2) A+a(d) \phi / 2} \delta^{D-d}(y),
\end{aligned}
$$


and the $m n$ components reduce to

$$
\begin{aligned}
& e^{d A+(\tilde{d}-2) B}\left[-\tilde{d} \partial^{m} \partial^{n} B+\delta^{m n} \tilde{d} \delta^{k l} \partial_{k} \partial_{l} B\right. \\
& -d \partial^{m} \partial^{n} A+d \delta^{m n} \delta^{k l} \partial_{k} \partial_{l} A-d \partial^{m} A \partial^{n} A+\frac{d(d+1)}{2} \delta^{m n} \delta^{k l} \partial_{k} A \partial_{l} A \\
& +d\left(\partial^{m} A \partial^{n} B+\partial^{m} B \partial^{n} A+(\tilde{d}-1) \delta^{m n} \delta^{k l} \partial_{k} A \partial_{l} B\right) \\
& \left.-\frac{1}{2} \partial^{m} \phi \partial^{n} \phi+\frac{1}{4} \delta^{m n} \delta^{k l} \partial_{k} \phi \partial_{l} \phi\right] \\
& -\frac{1}{2} e^{-d A+(\tilde{d}-2) B+2 C-a(d) \phi}\left[-\partial^{m} C \partial^{n} C+\frac{1}{2} \delta^{m n} \delta^{k l} \partial_{k} C \partial_{l} C\right] \\
& =0 .
\end{aligned}
$$

The antisymmetric tensor field equation (3.17) becomes

$$
\delta^{m n} \partial_{m}\left[e^{-a(d) \phi-d A+\tilde{d} B} \partial_{n} e^{C}\right]=2 \kappa^{2} T_{d} \delta^{D-d}(y),
$$

and the dilaton equation (3.18) becomes

$$
\begin{aligned}
& \delta^{m n} \partial_{m}\left(e^{d A+\tilde{d} B} \partial_{n} \phi\right)-\frac{a(d)}{2} e^{-d A+\tilde{d} B+2 C-a(d) \phi} \delta^{m n} \partial_{m} C \partial_{n} C \\
& =a(d) \kappa^{2} T_{d} e^{d A+a(d) \phi / 2} \delta^{(D-d)}(y) .
\end{aligned}
$$

Finally, the $(d-1)$-brane equation (3.19) becomes

$$
\partial_{m}\left(e^{d A+a(d) \phi / 2}-e^{C}\right)=0 .
$$

Hence we have five equations for the four unknown functions $A, B, C, \phi$ and the unknown parameter $a(d)$.

The unique solution, assuming that $g_{M N}$ tends asymptotically to $\eta_{M N}$, is given by

$$
\begin{gathered}
A=\frac{\tilde{d}}{2(d+\tilde{d})}\left(C-C_{0}\right), \\
B=-\frac{d}{2(d+\tilde{d})}\left(C-C_{0}\right), \\
\frac{a(d)}{2} \phi=\frac{a^{2}(d)}{4}\left(C-C_{0}\right)+C_{0},
\end{gathered}
$$

where $C_{0}=a \phi_{0} / 2$ and $\phi_{0}$ is the dilaton vev. $C$ is given by

$$
\begin{aligned}
e^{-C} & =e^{-C_{0}}+\frac{k_{d}}{y^{\tilde{d}}}, \quad \tilde{d}>0 \\
& =e^{-C_{0}}-\frac{\kappa^{2} T_{d}}{\pi} \ln y, \quad \tilde{d}=0
\end{aligned}
$$


and

$$
k_{d}=2 \kappa^{2} T_{d} / \tilde{d} \Omega_{\tilde{d}+1}
$$

where $\Omega_{\tilde{d}+1}$ is the volume of $S^{\tilde{d}+1}$. The parameter $a(d)$ is given by

$$
a^{2}(d)=4-\frac{2 d \tilde{d}}{d+\tilde{d}} .
$$

Note, incidentally, that for these solutions, the coefficients of the $\delta$-function in (3.30) and (3.33) vanish at $y=0$. So the Einstein equation and the dilaton equation are essentially source-free; only in the antisymmetric tensor equation is there a $\delta$-function source. We shall return to this in section 3.4 .

A crucial result of this section is that we have fixed the constant $a(d)$ as in (3.38) by the requirement that our theory (3.3) yield elementary $(d-1)$-brane solutions.

The mass per unit $(d-1)$-volume of the elementary $(d-1)$-brane is given by

$$
\mathcal{M}_{d}=\int d^{D-d} y \theta_{00}
$$

where $\theta_{M N}$ is the total energy-momentum pseudotensor of the combined gravity-matter system. We find

$$
\mathcal{M}_{d}=T_{d} e^{C_{0}}
$$

To compute the electric charge $e_{d}$ of (3.12) it is convenient to introduce polar coordinates

$$
y^{m}=\left(y, \theta^{i}\right)
$$

where $i=1, \ldots,(\tilde{d}+1)$, so that

$$
\delta_{m n} d y^{m} d y^{n}=d y^{2}+y^{2} d \Omega_{\tilde{d}+1}^{2}
$$

where $d \Omega_{\tilde{d}+1}^{2}$ is the metric on the unit $S^{\tilde{d}+1}$. Then we note from (3.29) that

$$
F_{y \mu_{1} \ldots \mu_{d}}=-\frac{1}{{ }^{g}} \varepsilon_{\mu_{1} \ldots \mu_{d}} \partial_{y} e^{C}
$$

The dual of $F,{ }^{*} F$, has non-vanishing components only in the $\theta^{i}$ directions

$$
\sqrt{-g}^{*} F^{\theta_{1} \ldots \theta_{D-d-1}}=-(-)^{(D-d)(d+1)} e^{2 C} \partial_{y} e^{-C}
$$


Hence, using (3.22),(3.35)-(3.37), we find

$$
e^{-a \phi *} F_{\theta_{1} \ldots \theta_{D-d-1}}=(-)^{(D-d)(d+1)} 2 \kappa^{2} T_{d} \frac{\varepsilon_{\theta_{1} \ldots \theta_{D-d-1}}}{\Omega_{\tilde{d}+1}} .
$$

It follows from (3.12) that

$$
e_{d}=\sqrt{2} \kappa T_{d}(-)^{(D-d)(d+1)},
$$

and hence

$$
\mathcal{M}_{d}=\frac{1}{\sqrt{2}}\left|e_{d}\right| e^{a(d) \phi_{0} / 2} .
$$

Thus we find that the mass and charge obey the same equality as we found for the supersymmetric solutions of section 2 even though as yet no supersymmetry has been assumed. This is in fact a consequence of assuming that the ratio of coefficients for the kinetic term and Wess-Zumino term in the $p$-brane $\sigma$-model are as given in (3.5).

There is a straightforward generalization to exact, stable multi- $(d-1)$-brane configurations obtained by a linear superposition of the solutions (3.36),

$$
e^{-C}=e^{-C_{o}}+\sum_{l} \frac{k_{d}}{\left|\vec{y}-\vec{y}_{l}\right|^{2}},
$$

where $\vec{y}_{l}$ corresponds to the position of each $(d-1)$-brane. To see the no-force condition explicitly, consider the multi- $(d-1)$-brane configuration (3.48) with, for example, $N(d-1)$ branes as sources. In general, we do not have the transverse $S O(D-d)$ symmetry, but we still have the $P_{d}$ Poincare symmetry for the configuration (3.48). Let each $(d-1)$-brane with label $l$ satisfy $X^{\mu}(l)=\xi^{\mu}$ so that, in particular, they all have the same orientation. The lagrangian for each of the $(d-1)$-branes with label $l$ in the fields of the sources given by (3.21)-(3.24) is, from (3.5)

$$
\mathcal{L}_{d}=-T_{d}\left[\sqrt{-\operatorname{det}\left(e^{2 A+a(d) \phi / d} \eta_{i j}+e^{2 B+a(d) \phi / d} \partial_{i} Y^{m}(l) \partial_{j} Y_{m}(l)\right.}-e^{C}\right]
$$

corresponding to a potential

$$
V=T_{d}\left(e^{d A+a(d) \phi / 2}-e^{C}\right),
$$

but this vanishes by (3.34). This generalizes to arbitrary $d$ and $D$ the no-force condition for strings and fivebranes discussed in section 2. Expanding out (3.49) we find

$$
\mathcal{L}=-\frac{T_{d}}{2} e^{(d-2) A+2 B+a(d) \phi / 2} \eta^{i j} \partial_{i} Y^{m} \partial_{j} Y_{m}+\ldots
$$

and so the absence of velocity-dependent forces corresponds to

$$
(d-2) A+2 B+a(d) \phi / 2=\text { constant },
$$

which is indeed satisfied by virtue of (3.35) and we find that the constant is just $C_{0}$. This generalizes to arbitrary $d$ and $D$, the absence of velocity dependent forces for strings and fivebranes in $D=10$ [20]. 


\subsection{The solitonic $(\tilde{d}-1)$-brane}

The elementary $(d-1)$-branes we have discussed so far correspond to solutions of the coupled field-brane system with action $I_{D}(d)+S_{d}$. As such they exhibit $\delta$-function singularities at $y=0$. They are characterized by a non-vanishing Noether electric charge $e_{d}$. By contrast, we now wish to find solitonic $(\tilde{d}-1)$-brane, corresponding to solutions of the source free equations resulting from $I_{D}(d)$ alone, which are regular at $y=0$, and which will be characterized by a non-vanishing topological magnetic charge $g_{\tilde{d}}$. (Recall that $\tilde{d}=D-d-2)$.

To this end, we now make an ansatz invariant under $P_{\tilde{d}} \times S O(D-\tilde{d})$. Hence we write (3.21) and (3.22) as before where now $\mu=0,1 \ldots(\tilde{d}-1)$ and $m=\tilde{d}, \tilde{d}+1, \ldots(D-1)$. The ansatz for the antisymmetric tensor, however, will now be made on the field strength rather than on the potential. From section 3.2 we recall that a non-vanishing electric charge corresponds to

$$
\frac{1}{\sqrt{2} \kappa} e^{-a \phi *} F_{\tilde{d}+1}=e_{d} \varepsilon_{\tilde{d}+1} / \Omega_{\tilde{d}+1},
$$

where $\varepsilon_{\tilde{d}+1}$ is the volume form on $S^{\tilde{d}+1}$. Accordingly, to obtain a non-vanishing magnetic charge, we make the ansatz

$$
\frac{1}{\sqrt{2} \kappa} F_{d+1}=g_{\tilde{d}} \varepsilon_{d+1} / \Omega_{d+1},
$$

where $\varepsilon_{d+1}$ is the volume form on $S^{d+1}$. Since this is an harmonic form, $F$ can no longer be written globally as the curl of $A$, but it satisfies the Bianchi identities. It is now not difficult to show that all the field equations of $I_{D}(d)$ are satisfied simply by making the replacement $d \rightarrow \tilde{d}$, and hence $a(d) \rightarrow a(\tilde{d})=-a(d)$ in (3.15)-(3.18) with the source terms set to zero. For future reference we write the explicit solution in the case $\phi_{0}=0$

$$
\begin{aligned}
d s^{2} & =\left(1+\frac{k_{\tilde{d}}}{y^{d}}\right)^{-d /(d+\tilde{d})} d x^{\mu} d x_{\mu}+\left(1+\frac{k_{\tilde{d}}}{y^{d}}\right)^{\tilde{d} /(d+\tilde{d})} d y_{m} d y_{m} \\
e^{2 \phi} & =\left(1+\frac{k_{\tilde{d}}}{y^{d}}\right)^{a(d)}, \\
F_{d+1} & =\sqrt{2} \kappa g_{\tilde{d}} \varepsilon_{d+1} / \Omega_{d+1}
\end{aligned}
$$

Note that by this device, we have found solutions everywhere including $y=0$, since the $\delta$-functions were already absent in the Einstein and dilaton equations. 
It follows that the mass per unit $(\tilde{d}-1)$-volume now satisfies

$$
\begin{aligned}
\mathcal{M}_{\tilde{d}} & =\frac{1}{\sqrt{2}}\left|g_{\tilde{d}}\right| e^{a(\tilde{d}) \phi_{0} / 2} \\
& =\frac{1}{\sqrt{2}}\left|g_{\tilde{d}}\right| e^{-a(d) \phi_{0} / 2}
\end{aligned}
$$

Note that the $\phi_{0}$ dependence is such that $\mathcal{M}_{\tilde{d}}$ is large for small $\mathcal{M}_{d}$ and vice-versa.

The electric charge of the elementary solution and the magnetic charge of the soliton solution obey a Dirac quantization rule $[27,28]$

$$
e_{d} g_{\tilde{d}}=2 \pi n, \quad n=\text { integer }
$$

and hence from (3.46)

$$
(-)^{(D-d)(d+1)} g_{\tilde{d}}=2 \pi n / \sqrt{2} \kappa T_{d}
$$

\subsection{Duality}

We now wish to consider the theory "dual" to (3.3) for which the roles of field equations (3.8) and Bianchi identities (3.9) are interchanged. To this end let us write the action

$$
\tilde{I}_{D}(\tilde{d})=\frac{1}{2 \kappa^{2}} \int d^{D} x \sqrt{-g}\left(R-\frac{1}{2}(\partial \phi)^{2}-\frac{1}{2(\tilde{d}+1) !} e^{a(d) \phi} \tilde{F}_{\tilde{d}+1}^{2}\right)
$$

where the $\operatorname{rank}(\tilde{d}+1)$ field strength $\tilde{F}$ is given by

$$
\tilde{F}_{\tilde{d}+1}=d \tilde{A}_{\tilde{d}}
$$

$a(d)$ is the same constant as appearing in (3.3) but appears with opposite sign, i.e

$$
a(\tilde{d})=-a(d)
$$

Allow these fields to couple to an elementary $\tilde{d}$-dimensional extended object (a" $(\tilde{d}-1)$ brane") with action

$$
\begin{aligned}
\tilde{S}_{\tilde{d}}=T_{\tilde{d}} \int d^{\tilde{d}} \xi( & -\frac{1}{2} \sqrt{-\gamma} \gamma^{i j} \partial_{i} X^{M} \partial_{j} X^{N} g_{M N} e^{-a(d) \phi / \tilde{d}}+\frac{(\tilde{d}-2)}{2} \sqrt{-\gamma} \\
& \left.-\frac{1}{\tilde{d} !} \varepsilon^{i_{1} i_{2} \ldots i_{\tilde{d}}} \partial_{i} X^{M_{1}} \partial_{i_{2}} X^{M_{2}} \ldots \partial_{i_{d}} X^{M_{\tilde{d}}} \tilde{A}_{M_{1} M_{2} \ldots M_{\tilde{d}}}\right) .
\end{aligned}
$$


The $\phi$ dependence is such that under the rescaling

$$
\begin{aligned}
g_{M N} & \rightarrow \tilde{\lambda}^{2 \tilde{d} /(D-2)} g_{M N}, \\
\tilde{A}_{M_{1} \ldots M_{\tilde{d}}} & \rightarrow \tilde{\lambda}^{\tilde{d}} \tilde{A}_{M_{1} \ldots M_{\tilde{d}},} \\
e^{\phi} & \rightarrow \tilde{\lambda}^{-2 \tilde{d}(D-\tilde{d}-2) /(D-2) a(d)} e^{\phi}, \\
\gamma_{i j} & \rightarrow \tilde{\lambda}^{2} \gamma_{i j},
\end{aligned}
$$

both actions scale the same way

$$
\begin{aligned}
\tilde{I}_{D}(\tilde{d}) & \rightarrow \tilde{\lambda}^{\tilde{d}} I_{D}(d), \\
\tilde{S}_{\tilde{d}} & \rightarrow \tilde{\lambda}^{\tilde{d}} \tilde{S}_{\tilde{d}} .
\end{aligned}
$$

The field equations and Bianchi identities of the $\tilde{A}$ field may be written

$$
\begin{gathered}
d^{*}\left(e^{a(d) \phi} \tilde{F}\right)=2 \kappa^{2}(-)^{\tilde{d}^{2} *} \tilde{J}, \\
d \tilde{F}=0 .
\end{gathered}
$$

It should be clear that the system described by $\tilde{I}_{D}(\tilde{d})+\tilde{S}_{\tilde{d}}$ admit the same elementary solutions as that described by $I_{D}(d)+S_{d}$ and that $\tilde{I}_{D}(\tilde{d})$ alone admits the same solitonic solutions as $I_{D}(d)$ alone, provided we everywhere make the replacement $d \rightarrow \tilde{d}$ and hence $a(d) \rightarrow a(\tilde{d})=-a(d)$. In particular the Noether electric charge is given by

$$
\tilde{e}_{\tilde{d}}=\frac{1}{\sqrt{2} \kappa} \int_{S^{d+1}} e^{a \phi *} \tilde{F}_{d+1},
$$

and the topological magnetic charge by

$$
\tilde{g}_{d}=\frac{1}{\sqrt{2} \kappa} \int_{S^{\tilde{d}+1}} \tilde{F}_{\tilde{d}+1},
$$

and they obey the condition

$$
\tilde{e}_{\tilde{d}} \tilde{g}_{d}=2 \pi n \text {. }
$$

So far we have discovered that the equations of $I_{D}(d)$ admit an elementary $(d-1)$ brane solution and a solitonic $(\tilde{d}-1)$-brane solution. Conversely, the equations of $\tilde{I}_{D}(\tilde{d})$ admit an elementary $(\tilde{d}-1)$-brane solution and a solitonic $(d-1)$-brane solution. We now wish to go a step further and assert that the $(d-1)$-brane is "dual" to the $(\tilde{d}-1)$-brane. In its strongest sense this means that the two theories are equivalent descriptions of the 
same physics. In the present context, however, we simply make the assumption that the $I_{D}(d)$ and $\tilde{I}_{D}(\tilde{d})$ are equivalent, i.e we assume that the metric $g_{M N}$ and dilaton $\phi$ are the same and that the $(\tilde{d}+1)$-form field strength $\tilde{F}_{\tilde{d}+1}$ is dual to the $(d+1)$-form field strength $F_{d+1}$. More precisely,

$$
\tilde{F}_{\tilde{d}+1}=e^{-a(d) \phi *} F_{d+1},
$$

so that the (source-free) field equations and Bianchi identities of $I_{D}(d),(3.8)$ and (3.9), become the Bianchi identities and (source-free) field equations of $\tilde{I}_{D}(\tilde{d}),(3.66)$ and $(3.65)$. This leads immediately to

$$
\begin{aligned}
& e_{d}=\tilde{g}_{d}, \\
& g_{\tilde{d}}=\tilde{e}_{\tilde{d}},
\end{aligned}
$$

and hence

$$
\kappa^{2} T_{d} T_{\tilde{d}}=|n| \pi
$$

The duality assumption also leads to a relation between the dimensionless loop expansion parameters of the $(d-1)$-brane and the $(\tilde{d}-1)$-brane. To see this we note that metrics appearing naturally in $(d-1)$-brane and $(\tilde{d}-1)$-brane $\sigma$-models $(3.5)$ and (3.62) are

$$
\begin{gathered}
g_{M N}(d)=e^{a(d) \phi / d} g_{M N}(\text { canonical }), \\
g_{M N}(\tilde{d})=e^{-a(d) \phi / \tilde{d}} g_{M N}(\text { canonical }) .
\end{gathered}
$$

If we rewrite $I_{D}(d)$ and $\tilde{I}_{D}(\tilde{d})$ in these variables we find

$$
\begin{aligned}
I_{D}(d)= & \frac{1}{2 \kappa^{2}} \int d^{D} x \sqrt{-g} e^{-(D-2) a(d) \phi / 2 d}[R \\
& \left.-\frac{1}{2}\left(1-\frac{a^{2}(D-1)(D-2)}{2 d^{2}}\right)(\partial \phi)^{2}-\frac{1}{2 \cdot(d+1) !} F_{d+1}^{2}\right],
\end{aligned}
$$

and

$$
\begin{aligned}
\tilde{I}_{D}(\tilde{d})= & \frac{1}{2 \kappa^{2}} \int d^{D} x \sqrt{-g} e^{(D-2) a(d) \phi / 2 \tilde{d}} R \\
& \left.-\frac{1}{2}\left(1-\frac{a^{2}(D-1)(D-2)}{2 \tilde{d}^{2}}\right)(\partial \phi)^{2}-\frac{1}{2(\tilde{d}+1) !} \tilde{F}_{\tilde{d}+1}^{2}\right] .
\end{aligned}
$$

Note that in both cases a common dilaton-dependent factor appears. This reveals that the $(d-1)$-brane loop counting parameter is

$$
\mathrm{g}_{d}=e^{(D-2) a(d) \phi_{0} / 4 d}
$$


and the $(\tilde{d}-1)$-brane loop counting parameter is

$$
\mathrm{g}_{\tilde{d}}=e^{-(D-2) a(d) \phi_{0} / 4 \tilde{d}}
$$

Hence

$$
\mathrm{g}_{d}^{d}=1 / \mathrm{g}_{\tilde{d}}^{\tilde{d}}
$$

and strongly coupled $(d-1)$ branes correspond to weakly coupled $(\tilde{d}-1)$ branes and vice-versa.

Finally we note that, in the case of $d=2$, the following field redefinition

$$
(D-2) a(2) \phi=8 \Phi
$$

yields from $(3.75)$ an $I_{D}(d)$ which is $D$-independent, namely

$$
I_{D}(2)=\frac{1}{2 \kappa^{2}} \int d^{D} x \sqrt{-g} e^{-2 \Phi}\left[R+4(\partial \Phi)^{2}-\frac{1}{2.3 !} F_{3}^{2}\right] .
$$

This is a well-known result in string theory. Curiously, there is no field redefinition which renders the integrand of $I_{D}(d)$ independent of $D$ for $d \neq 2$. However, we may dualize (3.81) to obtain

$$
\tilde{I}_{D}(D-4)=\frac{1}{2 \kappa^{2}} \int d^{D} x \sqrt{-g} e^{4 \Phi / D-4}\left[R-\frac{4(D-10)}{(D-4)^{2}}(\partial \Phi)^{2}-\frac{1}{2(D-3) !} \tilde{F}_{D-3}^{2}\right]
$$

In these string variables the metric of the elementary string is given by

$$
d s^{2}=\left(1+\frac{k_{2} e^{C_{0}}}{y^{D-4}}\right)^{-1} \eta_{\mu \nu} d x^{\mu} d x^{\nu}+\delta_{m n} d y^{m} d y^{n}
$$

with $\mu=0,1$ and $m=1 \ldots D-2$. Also

$$
a(2)=\sqrt{\frac{8}{D-2}},
$$

SO

$$
\Phi=\frac{1}{2}\left(C-C_{0}\right)+\frac{D-2}{4} C_{0},
$$

where

$$
\begin{aligned}
e^{-C} & =e^{-C_{0}}+\frac{k_{2}}{y^{D-4}}, \quad D>4 \\
& =e^{-C_{0}}-\frac{\kappa^{2} T_{2}}{\pi} \ln y \quad D=4 .
\end{aligned}
$$


On the other hand the solitonic $(D-5)$-brane is given by

$$
d s^{2}=\eta_{\mu \nu} d x^{\mu} d x^{\nu}+\left(1+\frac{k_{D-4}}{y^{2}} e^{C_{0}}\right) \delta_{m n} d y^{m} d y^{n}
$$

where $\mu=0 \ldots D-5$ and $m=D-4, \ldots, D-1$. Also

$$
a(D-4)=-\sqrt{\frac{8}{D-2}},
$$

so

$$
\Phi=-\frac{1}{2}\left(C-C_{0}\right)-\frac{(D-2)}{4} C_{0}
$$

where

$$
e^{-C}=e^{-C_{0}}+\frac{k_{D-4}}{y^{2}} .
$$

We note that in these string $\sigma$-model variables the transverse part of the metric in (3.83) is flat and the spacetime part of the metric in (3.87) is flat. These are therefore free field theories from the point of view of conformal field theory.

Having constructed the action in $\sigma$-model variables (3.75), it is instructive to compare it with Brans-Dicke theory. The action for Brans-Dicke gravity (generalized from 4 to $D$ dimensions) may be written in terms of a scalar field $\eta$ and some metric $g_{M N}(B D)$

$$
I(\text { Brans }- \text { Dicke })=\frac{1}{2 \kappa^{2}} \int d^{D} x \sqrt{-g}\left[\eta R-\frac{\omega}{\eta}(\partial \eta)^{2}\right]+\int d^{D} x \mathcal{L}(\text { matter }, g),
$$

where $\omega$ is a free parameter and where, by construction, $\mathcal{L}$ (matter, $g$ ) is independent of $\eta$. In comparing this to our general action $I_{D}(d)$ we have to decide what is meant by $\mathcal{L}$ (matter, $g$ ). Let us first suppose that this refers not to the antisymmetric tensor action of (3.3) but to the $(d-1)$-brane action $S_{d}$ of (3.5). Then we must make the identification

$$
g_{M N}(B D)=g_{M N}(d),
$$

where $g_{M N}(d)$ is the $(d-1)$-brane $\sigma$-model metric of (3.73). Comparison with (3.75) then yields the identifications

$$
\begin{gathered}
\eta=e^{-(D-2) a(d) \phi / 2 d}, \\
\omega=\frac{2 d^{2}}{(D-2)^{2} a^{2}(d)}-\frac{D-1}{D-2}=-\frac{(D-1)(d-2)-d^{2}}{(D-2)(d-2)-d^{2}},
\end{gathered}
$$

where we have used $a$. It is interesting to note, for example, that in $D=10$ strings $(d=2)$ correspond to $\omega=-1$, fivebranes $(d=6)$ to $\omega=0$ and threebranes $(d=4)$ to $\omega=\infty$. 


\subsection{The self-dual string and dyonic string in $D=6$}

The case $D=6$ is special because in this case the theory dual to the superstring is itself a superstring. The two strings are related by the strong/weak coupling replacement $\phi \rightarrow-\phi$. Compare (3.81) with (3.82), which are the bosonic sectors of $D=6$ supergravity and its dual. This permits the construction of a dyonic string which carries both electric and magnetic charges, which we shall shortly discuss.

However, there is another supersymmetric solitonic string in $D=6$ : the self-dual superstring which falls outside our previous discussions and requires a special treatment. This is the $D=6$ counterpart of the self-dual superthreebrane in $D=10$ of section 4 . Our starting point is the $N=2, D=6$ self-dual supergravity $[57,15]$ which, in common with the Type IIB superstring in $D=10$ discussed in section 4, admits covariant field equations, but no manifestly covariant field equations. It describes a graviton $e_{M}{ }^{A}$, two left-handed gravitini $\psi_{M a}$ and one tensor field $B_{M N}$ with self-dual field strength $G_{M N P}$. The gravitini transformation rules are (in our notation)

$$
\delta \psi_{M}=\nabla_{M} \varepsilon-\frac{1}{8} G_{M N P} \Gamma^{N P} \varepsilon .
$$

So if we make a two/four split as in section 3.2 with

$$
\begin{aligned}
\Gamma_{A} & =\left(\gamma_{\alpha} \otimes 1, \gamma^{3} \otimes \Sigma_{m}\right), \quad \Gamma^{7}=\gamma^{3} \otimes \Gamma^{5}, \\
\gamma^{3} & =\gamma^{0} \gamma^{1}, \quad \Gamma^{5}=\Sigma^{2} \Sigma^{3} \Sigma^{4} \Sigma^{5},
\end{aligned}
$$

the criterion for unbroken supersymmetry, $\delta \psi_{M}=0$, reduces to

$$
\begin{array}{r}
\partial_{\mu} \varepsilon-\frac{1}{2} \gamma^{3} \gamma_{\mu} \otimes \Sigma^{n}\left(\partial_{n} A+\frac{1}{2} e^{-2 A} \partial_{n} e^{C} \gamma^{3}\right) \varepsilon=0, \\
\partial_{m} \varepsilon+\frac{1}{2} \partial_{m} B \varepsilon-\frac{1}{2}\left(\delta^{n}{ }_{m}+\Sigma^{n}{ }_{m}\right)\left(\partial_{n} B-\frac{1}{2} e^{-2 A} \partial_{m} e^{C} \gamma^{3}\right) \varepsilon=0,
\end{array}
$$

and hence supersymmetry requires

$$
C=2 A, \quad B=-A, \quad \varepsilon=e^{-B / 2} \varepsilon_{0},
$$

where $\varepsilon_{0}$ obeys $\gamma^{3} \varepsilon_{0}=-\varepsilon_{0}$, and one half of the supersymmetries are broken.

The bosonic equations of motion are

$$
R_{M N}-\frac{1}{2} g_{M N} R=\frac{1}{4} G_{M}{ }^{P Q} G_{N P Q}
$$




$$
G_{M N P}=-\tilde{G}_{M N P},
$$

and substituting (3.98) yields

$$
e^{6 A} \delta^{m n} \partial_{m} \partial_{n} e^{-2 A}=0
$$

for the $\mu \nu$ components of the Einstein equation and

$$
e^{2 A} \delta^{m n} \partial_{m} \partial_{n} e^{-2 A}=0
$$

for the $m n$ components, so that

$$
e^{-2 A}=1+\frac{k_{2}}{y^{2}} .
$$

All the properties of the dyonic self-dual threebrane [58] to be discussed in section 4 apply, mutatis mutandis, to the dyonic self-dual string, including Dirac quantization rules and the saturation of the Bogolmol'nyi bound.

The effective bosonic equations of motion of this string are

$$
\begin{aligned}
& \partial_{i}\left(\sqrt{-\gamma} \gamma^{i j} \partial_{j} X^{N} g_{M N}\right)-\frac{1}{2} \sqrt{-\gamma} \gamma^{i j} \partial_{i} X^{N} \partial_{j} X^{P} \partial_{M} g_{N P} \\
& =\frac{1}{2} G_{M N P} \partial_{i} X^{N} \partial_{j} X^{P} \varepsilon^{i j}
\end{aligned}
$$

but, since $G_{M N P}=-\tilde{G}_{M N P}$, there is no manifestly covariant worldsheet action. It would be interesting to include the fermionic degrees of freedom and construct the spacetime supersymmetric, $\kappa$-symmetric, Green-Schwarz string equations, but this has not yet been done.

We shall now discuss the dyonic string solution [48] of the $D=6$ string action (3.81) and show that in the limit $e_{2}=g_{2}$ it reduces to the self-dual string configuration. The solution is

$$
\begin{aligned}
e^{-2 \Phi_{E}} & =1+\frac{k_{2}}{y^{2}}, \quad e^{2 \Phi_{M}}=1+\frac{\tilde{k}_{2}}{y^{2}}, \\
\Phi & =\Phi_{E}+\Phi_{M}, \\
d s^{2} & =e^{2 \Phi_{E}}\left(-d \tau^{2}+d \sigma^{2}\right)+e^{2 \Phi_{M}}\left(d y^{2}+y^{2} d \Omega^{2}\right), \\
G & =2 \tilde{k}_{2} \epsilon_{3}, \quad \tilde{G}=e^{-2 \Phi *} G=2 k_{2} \epsilon_{3},
\end{aligned}
$$

where $k_{2}=\kappa e_{2} / \sqrt{2} \Omega_{3}$ and $\tilde{k}_{2}=\kappa g_{2} / \sqrt{2} \Omega_{3}$. For $e_{2}=g_{2}$ (3.105) reduces to the selfdual string above. However, since the self-dual string was shown above to break $1 / 2$ of the supersymmetries of the self-dual theory, it breaks $3 / 4$ of the supersymmetries of the non-self-dual theory, a result that can be shown directly from the supersymmetry transformation rules of the non-self-dual theory even when $e_{2} \neq g_{2}$ [48]. One recovers $1 / 2$ of the supersymmetries when either $e_{2}$ or $g_{2}$ vanishes. 


\subsection{Singularity structure and interpolation}

In section 2.6, we had shown using test-probe computations and by examining curvature singularities that while the string is a singular solution of string theory and the fivebrane is a singular solution of fivebrane theory, the string and fivebrane are mutually nonsingular in the sense that each could be viewed as a nonsingular soliton of the other theory. This symmetry in the singularity structure was used to support the string/fivebrane duality conjecture. One might naively have expected that this state of affairs would generalize to arbitrary $p$-branes and their duals, but in this section we shall show that this is not the case. We find that only particles and strings are mutually nonsingular with their duals from the point of view of the test-probe/source approach, even though the absence of curvature singularities at $y=0$ persists for all $p$.

In probing the singularity structure, we consider the radial trajectory of the $(d-1)$ brane infalling into the dual $(\tilde{d}-1)$-brane background. We assume that the $(d-1)$-brane and $(\tilde{d}-1)$-brane are nonintersecting, as is generically the case for $D=d+\tilde{d}+2$. As before, the contribution of the antisymmetric field strength to the worldvolume action vanishes. The effective action reduces to

$$
\mathcal{L}=\sqrt{-\gamma}
$$

where $\gamma_{i j}=g_{M N} \partial_{i} X^{M} \partial_{j} X^{N}$ is the worldvolume metric and where $g_{M N}$ is the $(\tilde{d}-1)$-brane metric in the $(d-1)$-brane variables and is given by

$$
d s^{2}=\Delta(d)^{1-2 / d} \eta_{m u \nu} d x^{\mu} d x^{\nu}+\Delta(d)^{-2 / d} \delta_{m n} d y^{m} d y^{n}
$$

where $\Delta(d)=\left(1+(b / y)^{d}\right)^{-1}, \mu, \nu=0,1, \ldots, \tilde{d}-1$ are $(\tilde{d}-1)$-brane indices, $m, n=\tilde{d}, \ldots, D-1$ are transverse space indices and $y$ is the radial coordinate in the transverse space. A $(d-1)$ brane propagating in this background will in general have $n$ of its $d-1$ spatial directions parallel to the $(\tilde{d}-1)$-brane and $d-n-1$ directions perpendicular to the $(\tilde{d}-1)$-brane, where $n \leq n_{0}=\min (d-1, \tilde{d}-1)$.

Our criterion for the singularity of the $(\tilde{d}-1)$-brane is as follows: if for some $n$ a $(d-1)$ brane test-probe views the singularity at the $(\tilde{d}-1)$-brane source (i.e. the test-probe falls into the source in finite proper time) then the $(\tilde{d}-1)$-brane is viewed as a singular solution of $(d-1)$-brane theory. Otherwise, the dual solitons are mutually nonsingular, as was demonstrated for strings and fivebranes in particular. 
Replacing (3.107) in (3.106) for a given choice of $n$, the lagrangian reduces in the limit of radial motion to

$$
\mathcal{L}=\sqrt{\Delta^{n-1} \dot{t}^{2}-\Delta^{n-2} \dot{y}^{2}}
$$

It follows that

$$
E=\frac{\Delta^{n-1} \dot{t}}{\sqrt{\Delta^{n-1} \dot{t}^{2}-\Delta^{n-2} \dot{y}^{2}}}
$$

is a constant of the motion. We combine (3.109) with the geodesic condition $\gamma_{00}=-1$ for massive test-probes, which follows from setting $X^{0}=\tau$ on the worldsheet and compute the proper time for the radial fall of the test-probe into the dual source to be

$$
\tau=\int_{0}^{y_{0}} \frac{d y \Delta^{-1 / d}}{\sqrt{\Delta^{-n+1} E^{2}-1}},
$$

where $y_{0}$ is the initial radial separation. Note that for $n=0$ there is a turning point at $\Delta\left(y_{T}\right)=E^{2}$, so that no singularity can be observed (the force in this case being repulsive). So without loss of generality, we let $n \geq 1$ below. The question of singularity now reduces to whether or not $\tau$ in (3.110) converges or not near $y=0$. Convergence of the integral implies a finite proper time and a singular dual object while divergence implies infinite proper time and mutual nonsingularity of probe and source. Since $\Delta \rightarrow(y / b)^{d}$ as $y \rightarrow 0$, it is straightforward to show that finiteness of $\tau$ is equivalent to finiteness of

$$
I(d, n)=\int_{0}^{y_{0}} d y y^{d(n-1) / 2-1}
$$

$I$ is finite provided $d(n-1) / 2>0$, or $n>1$, since $d>0$. Since $n \leq n_{0}=\min (d-1, \tilde{d}-1)$, it follows that $I$ cannot be finite when either of the dual objects is a particle $(d=1)$ or a string $(d=2)$. This means that strings and particles are always mutually nonsingular with their duals, in whatever spacetime dimension they are embedded. If, however, $n_{0} \geq 2$, or in other words both $p$-branes are membranes or higher dimensional objects, then the choice $n=n_{0}$ yields finite $I$ and the dual objects are mutually singular. Note that this analysis does not depend on the spacetime dimension $D$ at all. Also note that in our earlier analysis for strings and fivebranes we set $n=n_{0}$, which in view of (3.111) and our singularity criterion is a consistent choice in the general case (since $n=n_{0}$ is the case in which $I$ is "most likely" to converge, and so that if there is a singularity it would certainly be observed in this case). 
Next we turn to the question of vacuum interpolation [46]. To determine the asymptotic form of the metric of near $y=0$ we introduce the new radial coordinate $r^{d}=y^{d}+a^{d}$ and let

$$
r=b\left(1+\frac{e^{d \rho / b}}{d}\right) .
$$

Near $r=b$ we get

$$
\begin{aligned}
d s^{2} & \sim e^{(d-2) \rho / b}\left(-d t^{2}+d \mathbf{x} \cdot d \mathbf{x}\right)+d \rho^{2}+b^{2} d \Omega_{d+1}^{2} \\
\phi & \sim-\frac{d a}{2 b} \rho \\
F_{d+1} & \sim d b^{d} \varepsilon_{d+1} .
\end{aligned}
$$

If $d \neq 2$ the asymptotic spacetime is $(a d S)_{\tilde{d}+1} \times S^{d+1}$, and there is an event horizon at $r=b$. If $d=2$ the dual $(\tilde{d}-1)$-brane interpolates between $D$-dimensional Minkowski spacetime and the product of $S^{3}$ with a $(\tilde{d}+1)$-dimensional Minkowski spacetime, generalizing the case of the $D=10$ fivebrane. For these cases the asymptotic behaviour of the dilaton near the $p$-brane core can be invariantly characterized as linear in an ignorable coordinate associated with a space-translation Killing vector.

\subsection{Blackbranes}

Finally we turn to the two-parameter solitonic solutions of the theory which display event horizons: the "blackbranes".

Using the canonical metric, the $(\tilde{d}-1)$-brane black soliton solution may be written for all $\tilde{d} \geq 1$ as

$$
\begin{aligned}
d s^{2}= & -\Delta_{+} \Delta_{-}^{-\tilde{d} /(d+\tilde{d})} d t^{2} \\
& +\Delta_{+}^{-1} \Delta_{-}^{\frac{a^{2}}{2 d}-1} d r^{2} \\
& +r^{2} \Delta_{-}^{\frac{a^{2}}{2 d}} d \Omega_{d+1}^{2} \\
& +\Delta_{-}^{\frac{d}{d+d}} d x^{i} d x_{i}, \quad i=1 \ldots \tilde{d}-1, \\
e^{-2 \phi}= & \Delta_{-}^{a}, \\
\Delta_{ \pm}= & {\left[1-\left(\frac{r_{ \pm}}{r}\right)^{d}\right], } \\
F_{d+1}= & d\left(r_{+} r_{-}\right)^{d / 2} \varepsilon_{d+1},
\end{aligned}
$$


where the magnetic charge $g_{\tilde{d}}$ and the mass per unit $(\tilde{d}-1)$-volume $\mathcal{M}_{\tilde{d}}$ are related to $r_{ \pm}$ by $[59]$

$$
g_{\tilde{d}}=\frac{\Omega_{d+1}}{\sqrt{2} \kappa} d\left(r_{+} r_{-}\right)^{d / 2}
$$

and

$$
\mathcal{M}_{\tilde{d}}=\frac{\Omega_{d+1}}{2 \kappa^{2}}\left[(d+1) r_{+}^{d}-r_{-}^{d}\right] .
$$

The solutions possess an $R \times S O(d+2) \times E(\tilde{d}-1)$ symmetry where $E(n)$ denotes the $n$-dimensional Euclidean group. The solutions exhibit an event horizon at $r=r_{+}$and an inner horizon at $r=r_{-}$. The absence of naked singularities, $r_{+} \geq r_{-}$, translates into the same Bogomol'nyi bound $\sqrt{2} \kappa \mathcal{M}_{\tilde{d}} \geq\left|\tilde{g}_{\tilde{d}}\right|$. In the special case $D=11, \tilde{d}=3,6$ they reduce to the black membrane and black fivebrane of [51]. In the special case $D=10$ i.e $\tilde{d}=8-d$, they reduce to the black $p$-brane solutions of [50]. In the special case $D=4, \tilde{d}=1$ they reduce to the Kaluza-Klein black hole solution of $[52,53,54,55]$. In the limit of zero charge, $r_{-}=0$, the dilaton and antisymmetric tensor are trivial and the metric reduces to

$$
d s^{2}=-\Delta_{+} d t^{2}+\Delta_{+}^{-1} d r^{2}+r^{2} d \Omega_{d+1}^{2}+d x^{i} d x_{i}
$$

In [60] it was argued that these solutions are classically unstable. More interesting is the extreme mass $=$ charge limit $r_{+}=r_{-}$where the metric component $g_{00}$ becomes equal to the one multiplying $d x^{i} d x^{i}$ and the symmetry is enlarged to $S O(d+2) \times P(\tilde{d})$. It is convenient to introduce the change of variables $y^{d}=r^{d}-r_{-}^{d}$, then (3.114) becomes

$$
\begin{aligned}
d s^{2} & =\Delta_{-}^{d /(d+\tilde{d})} d x^{\mu} d x_{\mu}+\Delta_{-}^{-\tilde{d} /(d+\tilde{d})}\left(d y^{2}+y^{2} d \Omega_{d+1}^{2}\right), \\
e^{-2 \phi} & =\Delta_{-}{ }^{a} \\
\Delta_{-} & =\left[1+\left(\frac{r_{-}}{y}\right)^{d}\right]^{-1}, \\
F_{d+1} & =d r_{-}^{d} \varepsilon_{d+1} .
\end{aligned}
$$

But in the case $1 \leq d \leq 7,1 \leq \tilde{d} \leq 7$, these are precisely the super $p$-branes, so $r_{+}=r_{-}$ also corresponds to the appearance of supersymmetry. It is also possible to find elementary black $(d-1)$-branes with parameters $\mathcal{M}_{d}$ and $e_{d}$ obeying the bound $\sqrt{2} \kappa \mathcal{M}_{d} \geq\left|e_{d}\right|$, by including a source term on the right hand side of the equations. In this case however, it would be necessary to relax the equality of the kinetic and WZW term coefficients in (3.5) to allow for mass $\neq$ charge. (This equality is forced on us in the supersymmetric case, by virtue of $\kappa$-symmetry [9]).

For specific values of $d$ and $D$, the extreme solutions also exhibit supersymmetry and hence stability is guaranteed. Some may be identified with the previously classified heterotic, Type IIA and Type IIB super $\tilde{p}$-branes. It is to this subject that we now turn. 


\section{The brane-scan revisited}

\subsection{Bose-Fermi matching: a necessary condition}

As pointed out in [19], the string configuration of chapter 2 also solves both the Type IIA and Type IIB supergravity equations. Once again, each breaks half of the spacetime supersymmetries. As discussed in section 4.3, the Type IIA solution may be shown to follow by simultaneous dimensional reduction of the $D=11$ supermembrane of section 4.2 The $N=1$ solution of section 2.1 then follows by truncation. Together with the $N=1$ fivebrane of section 2.4, all these solutions correspond to known points on the brane-scan of supersymmetric extended objects classified in [10] and discussed in the Introduction. According to this classification, no Type II fivebranes (or indeed any Type II $p$-branes with $p>1$ ) could exist. However, it was pointed out in [32] that the fivebrane configuration of chapter 2 also solves both the Type IIA and Type IIB supergravity equations and hence that Type II superfivebranes exist after all. Moreover, the Type IIB theory also admits a self-dual superthreebrane [58]. The no-go theorem is circumvented because in addition to the superspace coordinates $X^{M}$ and $\Theta^{\alpha}$ there are also higher spin fields on the worldvolume: vectors or antisymmetric tensors. This raises the question: are there other super $p$-branes and if so, for what $p$ and $D$ ?

We begin by asking what new points on the brane-scan are permitted by bose-fermi matching alone. There are surprisingly few: $p=5$ in $D=11 ; p=3,4, \ldots 9$ in $D=10$; $p=3,4,5$ in $D=6$ and $p=3$ in $D=4$. The much harder task is to narrow down these possibilities to objects that actually exist. One obvious handicap is that, unlike the $p$-branes discussed in [10] and in the Introduction, no-one has yet succeeded in writing down the action for these new Type II $p$-branes. The existence of the $p=3$ and $p=5$ objects mentioned above was established indirectly: by showing that they emerge as soliton solutions of either Type IIA or Type IIB supergravity. The nature of the worldvolume fields is then established by studying the zero modes of the soliton. In particular, a super $p$-brane requires that the soliton solution preserves some unbroken supersymmetry and hence that the zero modes form a supermultiplet. Although we know of no general proof that all supersymmetric extended objects correspond to a soliton, this is true of all those on the 
old brane-scan and thus seems a good guide to constructing the new one. Following this route we shall conclude that of all the possible $D=10$ Type II super $p$-branes permitted by bose-fermi matching alone, only those with $p=0$ (Type IIA), $p=1$ (Type IIA and IIB), $p=3$ (Type IIB), $p=4$ (Type IIA) $p=5$ (Type IIA and IIB) and $p=6$ (Type IIA) actually exist. [The reader may wonder why there seems to be a gap at $p=2$. Indeed, duality would seem to demand that in $D=10$ a Type IIA superfourbrane should imply a Type IIA supermembrane. This object does indeed exist but it should not be counted as a new theory since vectors are dual to scalars in $d=3$ and so its worldvolume action is simply obtained by dualizing one of the $11 X^{M}$ of the $D=11$ supermembrane.] Our results thus confirm the conjecture of [50] that super Type II $p$-brane solitons in $D=10$ exist for all $0 \leq p \leq 6$.

Given that the gauge-fixed theories display worldvolume supersymmetry, and given that we now wish to include the possibility of vector (and/or antisymmetric tensor) fields, it is a relatively straightforward exercise to repeat the bose-fermi matching conditions of the Introduction for vector (and/or antisymmetric tensor) supermultiplets. Once again, we may proceed in one of two ways. First, given that a worldvolume vector has $(d-2)$ degrees of freedom, the scalar multiplet condition (1.40) gets replaced by

$$
D-2=\frac{1}{2} m n=\frac{1}{4} M N
$$

Alternatively, we may simply list all the supermultiplets in the classification of [35] and once again interpret $D$ via (1.42). The results are shown in Fig. 2.

Several comments are now in order:

1) Vector supermultiplets exist only for $4 \leq d \leq 10$ [35]. In $d=3$ vectors have only 1 degree of freedom and are dual to scalars. So these multiplets will already have been included as scalar multiplets in section 1 . In $d=2$, vectors have no degrees of freedom.

2) The number of scalars in a vector supermultiplet is such that, from $(1.42), D=4,6$ or 10 only, in accordance with (4.1).

3) One must now repeat the analysis for antisymmetric tensors to see if any new points are introduced on the scan. For example in $d=6$ there is a chiral $(2,0)$ tensor supermultiplet, with a second rank tensor whose field strength is self-dual: $\left(B_{\mu \nu}^{-}, \lambda^{I}, \phi^{[I J]}\right)$, $I=1, \ldots, 4$, corresponding to the Type IIA fivebrane and a non-chiral $(1,1)$ vector multiplet $\left(B_{\mu}, \chi^{I}, A_{I}^{J}, \xi\right), I=1,2$, corresponding to the Type IIB fivebrane [32,33]. 
However, both occupy the same $(d=6, D=10)$ slot in Fig. 2. Nevertheless, there is a new point on the scan, $(d=6, D=11)$, namely the $D=11$ superfivebrane, the corresponding $d=6$ supermultiplet being identical to that of the Type IIA fivebrane. This last observation corrects an omission in [61].*

4) We emphasize that Fig. 2 merely tells us what is allowed by bose/fermi matching. We must now try to establish which of these possibilities actually exists.

All of the circles on the brane-scan are known to correspond to soliton solutions of an underlying supersymmetric field theory [8,18,19,24,23,29,30]. As for the crosses, supersymmetric soliton solutions of both Type IIA and Type IIB supergravity have been found for the case $(d=6, D=10)$ [32] and of Type IIB for $(d=4, D=10)$ [58] and of $N=1$ supergravity for the case $(d=6, D=11)[51]$. What about the others? In the next section we shall show that in $D=10$ they exist for worldvolume dimensions $d=1$ (Type IIA), $d=2$ (Types IIA and IIB), $d=3$ (Type IIA), $d=4$ (Type IIB), $d=5$ (Type IIA), $d=6$ (Types IIA and IIB) and $d=7$ (Type IIA).

$\begin{array}{cccccc}d & \tilde{d} & \alpha(d) & A & B & \phi \\ 1 & 7 & -3 / 2 & 7 C / 16 & -C / 16 & -3 C / 4 \\ 2 & 6 & 1 & 3 C / 8 & -C / 8 & C / 2 \\ 3 & 5 & -1 / 2 & 5 C / 16 & -3 C / 16 & -C / 4 \\ 4 & 4 & 0 & C / 4 & -C / 4 & 0 \\ 5 & 3 & 1 / 2 & 3 C / 16 & -5 C / 16 & C / 4 \\ 6 & 2 & -1 & C / 8 & -3 C / 8 & -C / 2 \\ 7 & 1 & 3 / 2 & C / 16 & -7 / 16 & 3 C / 4\end{array}$

Table 2. The functions $A, B$ and $\phi$ in terms of $C$ as demanded by supersymmetry.

\subsection{Type II $(d-1)$-branes}

Let us begin in $D=10$ with Type IIA supergravity, whose bosonic action is given by

$$
\begin{aligned}
I_{10}(I I A) & =\frac{1}{2 \kappa^{2}} \int d^{10} x \sqrt{-g}\left[R-\frac{1}{2}(\partial \phi)^{2}-\frac{1}{2.3 !} e^{-\phi} F_{3}^{2}\right. \\
& \left.-\frac{1}{2.2 !} e^{3 \phi / 2} F_{2}^{2}-\frac{1}{2.4 !} e^{\phi / 2} F_{4}^{2}\right] \\
& -\frac{1}{4 \kappa^{2}} \int F_{4} \wedge F_{4} \wedge A_{2},
\end{aligned}
$$

* We are grateful to Paul Townsend for pointing this out. 
where

$$
F_{4}^{\prime}=d A_{3}+A_{1} \wedge F_{3}
$$

From (3.38) we see that the kinetic terms for gravity, dilaton and antisymmetric tensors are also correctly described by the generic action $I_{10}(d)$ of $(3.3)$ with $d=1,2,3$ (i.e $\left.\tilde{d}=7,6,5\right)$. Both the elementary string $(d=2)$ and fivebrane $(d=6)$ solutions of $N=1$ supergravity described above continue to provide solutions to Type IIA supergravity, as may be seen by setting $F_{2}=F_{4}=0$. [This observation is not as obvious as it may seem in the case of the elementary fivebranes or solitonic strings, however, since it assumes that one may dualize $F_{3}$. Now the Type IIA action follows by dimensional reduction from the action of $D=11$ supergravity which contains $F_{4}$. There exists no dual of this action in which $F_{4}$ is replaced by $F_{7}$ essentially because $A_{3}$ appears explicitly in the Chern-Simons term $F_{4} \wedge F_{4} \wedge A_{3}$ [62]. Since $F_{4}$ and $F_{3}$ in $D=10$ originate from $F_{4}$ in $D=11$, this means that we cannot simultaneously dualize $F_{3}$ and $F_{4}$ but one may do either separately. ${ }^{\dagger}$ By partial integration one may choose to have no explicit $A_{3}$ dependence in the Chern-Simons term of (4.18) or no explicity $A_{2}$ dependence, but not both.] Furthermore, by setting $F_{2}=F_{3}=0$ we find elementary membrane $(d=3)$ and solitonic fourbrane $(\tilde{d}=5)$ solutions, and then by dualizing $F_{4}$, elementary fourbrane $(d=5)$ and solitonic membrane $(\tilde{d}=3)$ solutions. Finally, by setting $F_{3}=F_{4}=0$, we find elementary particle $(d=1)$ and solitonic sixbrane $(\tilde{d}=7)$ solutions and then by dualizing $F_{2}$, elementary sixbrane $(d=7)$ and solitonic particle $(\tilde{d}=1)$ solutions.

Next we consider Type IIB supergravity in $D=10$ whose bosonic sector consists of the graviton $g_{M N}$, a complex scalar $\phi$, a complex 2-form $A_{2}$ (i.e with $d=2$ or, by duality $d=6$ ) and a real 4 -form $A_{4}$ (i.e with $d=4$ which in $D=10$ is self-dual). Because of this self-duality of the 5 -form field strength $F_{5}$, there exists no covariant action principle of the kind (3.3) and, strictly speaking, our previous analysis ceases to apply. Nevertheless we can apply the same logic to the equations of motion and we find that the solution again falls into the generic category (3.35)-(3.38). First of all, by truncation it is easy to see that the same string $(d=2)$ and fivebrane $(d=6)$ solutions of $N=1$ supergravity continue to solve the field equations of Type IIB. On the other hand, if we set to zero $F_{3}$ and solve the self-duality condition $F_{5}=-{ }^{*} F_{5}$ then we find the special case of $(3.35)$ with $d=\tilde{d}=4$ and hence $\alpha=0$ and $\phi=0$. This is the self-dual superthreebrane [58] discussed in more detail below.

$\dagger$ We are grateful to H. Nishino for this observation. 
All of the above elementary and solitonic solutions satisfy the mass = charge conditions (3.47) and (3.56). Our next task is to check for supersymmetry. We begin by making the same ansatz as in section 3.3, namely (3.21)-(3.23) but this time substitute into the supersymmetry transformation rules rather than the field equations, and demand unbroken supersymmetry. This reduces the four unknown functions $A, B, C$ and $\phi$ to one. We then compare the results with the known solutions.

For Type IIA supergravity with vanishing fermion background, the gravitino transformation rule is

$$
\begin{aligned}
\delta \psi_{M}=D_{m} \varepsilon & +\frac{1}{64} e^{3 \phi / 4}\left(\Gamma_{M} M_{1} M_{2}-14 \delta_{M}{ }^{M_{1}} \Gamma^{M_{2}}\right) \Gamma^{11} \varepsilon F_{M_{1} M_{2}} \\
& +\frac{1}{96} e^{-\phi / 2}\left(\Gamma_{M} M_{1} M_{2} M_{3}-9 \delta_{M}{ }^{M_{1}} \Gamma^{M_{2} M_{3}}\right) \Gamma^{11} \varepsilon F_{M_{1} M_{2} M_{3}} \\
& +\frac{i}{256} e^{\phi / 4}\left(\Gamma_{M}{ }^{M_{1} M_{2} M_{3} M_{4}}-\frac{20}{3} \delta_{M}^{M_{1}} \Gamma^{M_{2} M_{3} M_{4}}\right) \varepsilon F_{M_{1} M_{2} M_{3} M_{4}}
\end{aligned}
$$

and the dilatino rule is

$$
\begin{aligned}
\delta \lambda=\frac{1}{4} \sqrt{2} D_{M} & \phi \Gamma^{M} \Gamma^{11} \varepsilon+\frac{3}{16} \frac{1}{\sqrt{2}} e^{3 \phi / 4} \Gamma^{M_{1} M_{2}} \varepsilon F_{M_{1} M_{2}} \\
& +\frac{1}{24} \frac{i}{\sqrt{2}} e^{-\phi / 2} \Gamma^{M_{1} M_{2} M_{3}} \varepsilon F_{M_{1} M_{2} M_{3}} \\
& -\frac{1}{192} \frac{i}{\sqrt{2}} e^{\phi / 4} \Gamma^{M_{1} M_{2} M_{3} M_{4}} \varepsilon F_{M_{1} M_{2} M_{3} M_{4}},
\end{aligned}
$$

where $\Gamma^{M}$ are the $D=10$ Dirac matrices, where the covariant derivative is given by

$$
D_{M}=\partial_{M}+\frac{1}{4} \omega_{M A B} \Gamma^{A B}
$$

with $\omega_{M A B}$ the Lorentz spin connection, where

$$
\Gamma^{M_{1} M_{2} \ldots M_{n}}=\Gamma^{\left[M_{1}\right.} \Gamma^{M_{2}} \ldots \Gamma^{\left.M_{n}\right]}
$$

and where

$$
\Gamma^{11}=i \Gamma^{0} \Gamma^{1} \ldots \Gamma^{9} .
$$

Similarly the Type IIB rules are

$$
\begin{aligned}
\delta \psi_{M}=D_{M} \varepsilon & +\frac{i}{4 \times 480} \Gamma^{M_{1} M_{2} M_{3} M_{4}} \Gamma_{M} \varepsilon F_{M_{1} M_{2} M_{3} M_{4}} \\
& +\frac{1}{96}\left(\Gamma_{M}{ }^{M_{1} M_{2} M_{3}}-9 \delta_{M}{ }^{M_{1}} \Gamma^{M_{2} M_{3}}\right) \varepsilon^{*} F_{M_{1} M_{2} M_{3}}
\end{aligned}
$$


and

$$
\delta \lambda=i \Gamma^{M} \varepsilon^{*} P_{M}-\frac{1}{24} i \Gamma^{M_{1} M_{2} M_{3}} \varepsilon F_{M_{1} M_{2} M_{3}}
$$

where

$$
P_{M}=\partial_{M} \phi /\left(1-\phi^{*} \phi\right)
$$

In the Type IIB case, $\varepsilon$ is chiral

$$
\Gamma_{11} \varepsilon=\varepsilon
$$

The requirement of unbroken supersymmetry is that there exist Killing spinors $\varepsilon$ for which both $\delta \psi_{M}$ and $\delta \lambda$ vanish. Substituting our ansatze into the transformation rules we find that for every $1 \leq d \leq 7$ there exist field configurations which break exactly half the supersymmetries. This is just what one expects for supersymmetric extended object solutions $[8,18,19,24,23,29]$ and is intimately related to the $\kappa$-symmetry discussed in the Introduction and the Bogomoln'yi bounds. The corresponding values of $A, B$ and $\phi$ in terms of $C$ are given in Table 2. The important observation, from (3.35), is that the values required by supersymmetry also solve the field equations. Thus in addition to the $D=10$ super $(d-1)$ branes already known to exist for $d=2$ (Heterotic, Type IIA and Type IIB), $d=4$ (Type IIB only) and $d=6$ (Heterotic, Type IIA and Type IIB), we have established the existence of a Type IIA superparticle $(d=1)$, a Type IIA supermembrane $(d=3)$, a Type IIA superfourbrane $(d=5)$ and a Type IIA supersixbrane $(d=7)$.

It is perhaps worth saying a few more words about the self-dual superthreebrane. By virtue of the (anti) self-duality condition $F_{5}=-{ }^{*} F_{5}$, the electric Noether charge coincides with the topological magnetic charge

$$
e_{4}=-g_{4}
$$

(Note that such a condition is possible only in theories allowing a real self-duality condition i.e. in 2 mod 4 dimensions, assuming Minkowski signature. The self-dual string of section 3.6 is another example.) The usual Dirac quantization rule for $(d-1)$ branes (3.57) may, following $[63,64,65]$, be generalized to dyons carrying both electric and magnetic charges. In $D$ spacetime dimensions, a $(d-1)$-brane with charges $\left(e_{1 d}, g_{1} \tilde{d}\right)$ is related to another with charge $\left(e_{2 d}, g_{2} \tilde{d}\right)$ by

$$
e_{1 d} g_{2 \tilde{d}}-e_{2 d} g_{1 \tilde{d}}=2 \pi n
$$

Note, however, that this by itself says nothing about the quantization of the product $e_{1 d} g_{1 \tilde{d}}$. (Witten [66] has provided such a dyon quantization rule, but it requires either 
$T$ invariance, which is violated by the self-duality condition, or else an action principle, which is also absent for Type IIB supergravity.) So we cannot combine (4.13) and (4.14) to obtain $e_{4}$ and $g_{4}$ as pure numbers.

Finally we count bosonic and fermionic zero modes. We know that one half of the supersymmetries are broken, hence we have 16 fermionic zero modes. Regrouping these 16 fermionic zero modes, we get four Majorana spinors in $d=4$. Hence the $d=4$ worldvolume supersymmetry is $N=4$. Worldvolume supersymmetry implies that the number of fermionic and bosonic on-shell degrees of freedom must be equal, so we need a total of eight bosonic zero modes. There are the usual six bosonic translation zero modes, but we are still short of two. The two extra zero modes come from the excitation of the complex antisymmetric field strength $G_{M N P}$. The equation of motion for small fluctuations of the two-form potential $b$ in the soliton background is

$$
D^{P} G_{M N P}=-\frac{i}{6} F_{M N P Q R} G^{P Q R} .
$$

This is solved by

$$
\begin{aligned}
& b=e^{i k \cdot x} E \wedge d e^{2 A}, \\
& G=d b+i *(d b),
\end{aligned}
$$

where $k$ is a null vector in the two Lorentzian dimensions tangent to the worldvolume. $E$ is a constant polarization vector orthogonal to $k$ but tangent to the worldvolume and $*$ the Hodge dual in the worldvolume directions. Although $G$ is a complex tensor, the zeromodes solution gives only one real vector field on the worldvolume which provides the other two zero modes. These two zero modes turn out to be pure gauge at zero worldvolume momentum. Together with the other zero modes, these fields make up the $d=4, N=4$ matter supermultiplet $\left(A_{\mu}, \lambda^{I}, \phi^{[I J]}\right)$.

Note that in the self-dual case the condition (3.79) simply becomes

$$
\mathrm{g}_{d}^{d}=1 / \mathrm{g}_{d}^{d}=1 .
$$

We note as in [50] that, by virtue of its dimensionality, this self-dual threebrane comes closest to realizing the old idea of "spacetime as a membrane".

We have classified all supersymmetric extended objects in $D=10$ that correspond to solitons of a Poincaré supersymmetric field theory in the usual spacetime signature which break half the spacetime supersymmetries. We cannot at the present time rigorously rule 
out the existence of other super $p$-branes which do not correspond to solitons. However, we regard their existence as unlikely. Further progress would require that we construct the spacetime Green-Schwarz supersymmetric and $\kappa$-symmetric actions for these new Type II $p$-branes and, to date, this has not been done. All we know is that, in a physical gauge, the worldvolume theory corresponding to the zero modes of the soliton is described by vector or antisymmetric tensor supermultiplet as in Table 3.

$$
\begin{array}{ccccc}
d=7 & \text { Type IIA } & \left(A_{\mu}, \lambda, 3 \phi\right) & & n=1 \\
d=6 & \text { Type IIA } & \left(B_{\mu \nu}^{-}, \lambda^{I}, \phi^{[I J]}\right) & I=1, \ldots, 4 & \left(n_{+}, n_{-}\right)=(2,0) \\
& \text { Type IIB } & \left(B_{\mu}, \chi^{I}, A^{I}{ }_{J}, \xi\right) & I=1,2 & \left(n_{+}, n_{-}\right)=(1,1) \\
d=5 & \text { Type IIA } & \left(A_{\mu}, \lambda^{I}, \phi^{[I J]}\right) & I=1, \ldots, 4 & n=2 \\
d=4 & \text { Type IIB } & \left(B_{\mu}, \chi^{I}, \phi^{[I J]}\right) & I=1, \ldots, 4 & n=4 \\
d=3 & \text { Type IIA } & \left(\chi^{I}, \phi^{I}\right) & I=1, \ldots, 8 & n=8 \\
d=2 & \text { Type IIA } & \left(\lambda_{L}^{I}, \phi_{L}^{I}\right) & I=1, \ldots, 16 & \left(n_{+}, n_{-}\right)=(16,0) \\
& \text { Type IIB } & \left(\chi_{L}^{I}, \phi_{L}^{I}\right),\left(\chi_{R}^{I}, \phi_{R}^{I}\right) & I=1, \ldots, 8 & \left(n_{+}, n_{-}\right)=(8,8)
\end{array}
$$

Table 3: Gauge-fixed theories on the worldvolume, corresponding to the zero modes of the soliton, are described by the above supermultiplets.

In our classification, we have also omitted supersymmetric solitons which break more than half the supersymmetries since these solutions presumably admit no $\kappa$-symmetric Green-Schwarz action (at least, not of the kind presently known). Examples of this are provided by the $D=10$ double-instanton string of [67] (which breaks $3 / 4$ ), the $D=10$ octonionic string of [68] (which breaks 15/16), and the $D=11$ extreme black fourbrane of [69] and extreme black sixbrane of [51] (which break 3/4 and 7/8, respectively).

Finally, we ask what are the implications of our results for the idea of duality, in the sense that one theory is simply providing a dual description of the same physics of another theory with the weak-coupling regime of one being the strong-coupling of the other? At the classical level discussed in this section, we see that in $D=10$ supersymmetry has narrowed down the possibilities to just four, namely particle/sixbrane duality (Type IIA only), string/fivebrane duality (Heterotic, Type IIA or Type IIB), membrane/fourbrane duality (Type IIA only) and threebrane self-duality (Type IIB only).

Although strictly speaking $D=11$ lies outside the realm of superstring theory, the $D=11$ supersymmetric extended objects serve the purpose of completing the brane-scan and of illustrating the utility of simultaneous dimensional reduction. Before turning our 
attention to $N=1, D=11$ supergravity, however, it is convenient to make the replacement (3.35) in $(3.75)$ so that

$$
\begin{aligned}
I_{D}(d)= & \frac{1}{2 \kappa^{2}} \int d^{D} x \sqrt{-g} e^{-(D-2) a^{2} C / 4 d}[R \\
& \left.-\frac{a^{2}}{8}\left(1-\frac{a^{2}(D-1)(D-2)}{2 d^{2}}\right)(\partial C)^{2}-\frac{1}{2 \cdot(d+1) !} F_{d+1}^{2}\right]
\end{aligned}
$$

where we have set $C_{0}=0$ for simplicity. If we now focus on the case $(D=11, d=3)$ we find from (3.38) that

$$
a(3)=0,
$$

and hence

$$
I_{11}(3)=\frac{1}{2 \kappa^{2}} \int d^{11} x \sqrt{-g}\left[R-\frac{1}{2.4 !} F_{4}^{2}\right] .
$$

This is to be compared with the bosonic sector of $D=11$ supergravity

$$
I(D=11 S U G R A)=\frac{1}{2 \kappa^{2}} \int d^{11} x \sqrt{-g}\left[R-\frac{1}{2.4 !} F_{4}^{2}\right]+\frac{1}{12 \kappa^{2}} \int F_{4} \wedge F_{4} \wedge A_{3} .
$$

As discussed above, there is no dualized form of this action since $A_{3}$ enters explicitly. We can however find an elementary membrane solution. Once again, this is just the $d=3$, $\tilde{d}=6, a(3)=0$ special case of our general solutions (3.35)-(3.38). This is the solution of [23] which breaks half the supersymmetries and corresponds to the eleven-dimensional supermembrane of [9], for which there is a covariant $\kappa$-symmetric Green-Schwarz action. This theory also exhibits a soliton solution which is the $\tilde{d}=6, d=3$ superfivebrane of [51], for which no such Green-Schwarz action is known.

\subsection{Simultaneous dimensional reduction}

Simple dimensional reduction allows us to derive the actions $I_{D}(d)$ and $S_{d}$ for a $(d-1)$ -

brane moving in a $D$-dimensional spacetime from the actions $I_{D+1}(d)$ and $S_{d}$ corresponding to a $(d-1)$-brane in a $(D+1)$-dimensional spacetime. This corresponds to

$$
\begin{aligned}
D+1 & \rightarrow D, \\
d & \rightarrow d, \\
\tilde{d}+1 & \rightarrow \tilde{d},
\end{aligned}
$$


and takes us vertically on the brane-scan. Double dimensional reduction [70], on the other hand, allows us to derive the actions $I_{D}(d)$ and $S_{d}$ for a $(d-1)$-brane moving in $D$-dimensional spacetime from the actions $I_{D+1}(d+1)$ and $S_{d+1}$. This corresponds to

$$
\begin{aligned}
D+1 & \rightarrow D, \\
d+1 & \rightarrow d, \\
\tilde{d} & \rightarrow \tilde{d},
\end{aligned}
$$

and takes us diagonally on the brane-scan. The first example of this was to rederive the Type IIA superstring in $D=10$ from the supermembrane in $D=11$ [70]. This process thus allows us, for example, to rederive the elementary string of section 2.1 in $D=10$ from the $D=11$ supermembrane discussed above.

To see how it works in general, let us denote all $(D+1, d+1)$-dimensional quantities by a hat and all $(D, d)$ dimensional quantities without. Then with

$$
\begin{aligned}
\hat{X}^{\hat{M}} & =\left(X^{M}, X^{d}\right), \quad M=0,1, \ldots,(d-1),(d+1), \ldots, D-1 \\
\hat{\xi}^{\hat{\mu}} & =\left(\xi^{i}, \xi^{d}\right),
\end{aligned}
$$

double dimensional reduction consists in setting

$$
\xi^{d}=X^{d}
$$

taking $X^{d}$ to be the coordinate on a circle of radius $R$, and discarding all but the zero modes. In practice, this means taking the background fields $\hat{\phi}, \hat{g}_{\hat{M} \hat{N}}$ and $\hat{A}_{\hat{M} \hat{N} \ldots \hat{M}_{d}}$ to be independent of $X^{d}$. To recover $S_{d}$, with only background fields $\phi, g_{M N}$ and $A_{M_{1} M_{2} \ldots M_{d-1}}$, a further truncation is necessary. Specifically we write

$$
\hat{g}_{\hat{M} \hat{N}}(\sigma-\text { model })=e^{-2 \beta \phi / d+1}\left(\begin{array}{cc}
g_{M N}(\sigma-\text { model }) & 0 \\
0 & e^{2 \beta \phi}
\end{array}\right)
$$

where $\beta$ is a, for the moment, arbitrary constant and

$$
\hat{A}_{012 \ldots d+1}=A_{012 \ldots d}
$$

with other components set to zero. The condition (4.27) ensures from (3.20) that

$$
\sqrt{-\hat{\gamma}}=\sqrt{-\gamma}
$$


and hence, together with condition (4.28), we recover the correct $\sigma$-model action for $S_{d-1}$ starting from $\hat{S}_{d}$ provided

$$
2 \pi R \hat{T}_{d+1}=T_{d}
$$

We fix $\beta$ and the relation between $\hat{\phi}$ and $\phi$ by requiring that we obtain the correct background field action $I_{D}(d)$ starting from $I_{D+1}(d+1)$. So from $(3.75)$

$$
\begin{aligned}
& e^{-(D-1) \hat{a} \hat{\phi} / 2(d+1)} \sqrt{-\hat{g}}\left[\hat{R}-\frac{1}{2}\left(1-\frac{\hat{a}^{2}(D(D-1)}{2(d+1)^{2}}\right)(\partial \hat{\phi})^{2}\right] \\
& =e^{-(D-2) a \phi / 2 d} \sqrt{-g}\left[R-\frac{1}{2}\left(1-\frac{a^{2}(D-1)(D-2)}{2 d^{2}}\right)(\partial \phi)^{2}\right]
\end{aligned}
$$

which gives

$$
\begin{aligned}
\hat{\phi} & =\delta \phi \\
\frac{(D-1) \hat{a}}{2(d+1)} \delta= & \frac{(D-2) a}{2 d}-\frac{\tilde{d} \beta}{d+1} \\
1-\frac{a^{2}(D-1)(D-2)}{2 d^{2}}= & \delta^{2}\left(1-\frac{\hat{a}^{2} D(D-1)}{2(d+1)^{2}}\right) \\
& -4 \beta \frac{\tilde{d}+1}{d+1} \frac{(D-2) a}{2 d} \\
& +2 \beta^{2} \frac{D(D-1)-2(d+1)(\tilde{d}+1)}{(d+1)^{2}}
\end{aligned}
$$

and hence

$$
\begin{gathered}
\beta=\frac{2}{d a}, \\
\delta=\frac{\hat{a}}{a}
\end{gathered}
$$

from solving eqs. (4.32). We also require

$$
\hat{\kappa}^{2}=2 \pi R \kappa^{2}
$$

Note that the Dirac quantization rule (3.72) involving $\kappa^{2}$ and $T$ follows from that involving $\hat{\kappa}^{2}$ and $\hat{T}$ on using (4.30) and (4.35). In canonical variables, we have

$$
\begin{aligned}
\hat{g}_{M N}(\text { canonical }) & =e^{-2 \tilde{d} \phi / a(d)(d+\tilde{d})(d+1+\tilde{d})} g_{M N}(\text { canonical }) \\
\hat{g}_{d d}(\text { canonical }) & =e^{2 \tilde{d} \phi /(d+1+\tilde{d}) a(d)}
\end{aligned}
$$


As an application of simultaneous dimensional reduction, we may derive the elementary string solution in $D=10$ from the $D=11$ membrane solution of [23]. The $D=10$ fields $g_{M N}, A_{M N}$ and $\phi$ are given by

$$
\begin{aligned}
\hat{g}_{M N} & =e^{-\phi / 6} g_{M N}(\text { canonical }) \\
\hat{g}_{22} & =e^{4 \phi / 3} \\
\hat{A}_{012} & =A_{01}
\end{aligned}
$$

[Curiously, the metric $\hat{g}_{M N}$ in (4.37) bears the same relation to $g_{M N}$ (canonical) as does the fivebrane $\sigma$-model metric in (3.74) since $a(d=2)=1$ and $\tilde{d}=6$. This phenomenon happens in general whenever $\hat{a}=0$ i.e for $(d+1=3, \tilde{d}=6),(d+1=4, \tilde{d}=4)$ and $(d+1=$ $6, \tilde{d}=3)]$. Similarly starting from the sixbrane in $D=10$ we may proceed diagonally down the brane-scan to a particle in $d=4$. It is not difficult to show that the solutions so obtained will continue to preserve exactly one half of the supersymmetries. Starting from the $d \leq 7$ solutions in $D=10$ we can thus fill out the triangle of supersymmetric extended objects shown in Fig. 3. At first sight, this seems to contradict Fig. 2 since solutions appear where no supermultiplet is allowed. The resolution is simply that only the cases $d=1,3,4,5,6$ and 7 in $D=10$ are fundamental. All the others are obtained by simply dimensional reduction of these or the $D=11$ supermembrane, and are thus described by the same gauge-fixed action.

Finally, it is shown in [45] that in the special cases $\tilde{d}=3, d=6 ; \tilde{d}=6, d=3$ and $\tilde{d}=4, d=4$ (all of which, as remarked above, have $a=0$ ) the endpoints of the interpolation, namely Minkowski space $M_{D}$ at $y=\infty$ and $(A d S)_{\tilde{d}+1} \times S^{d+1}$ at $y=0$, are maximally supersymmetric. 


\section{Heterotic strings and fivebranes in $\mathbf{D}=\mathbf{1 0}$}

\subsection{Inclusion of Yang-Mills fields}

In this section we focus on soliton solutions of the heterotic string [25]. Of particular importance is the heterotic fivebrane of [24], which did much to lend credence to the conjecture of string/fivebrane duality [17],[24]. The field theory limit of the heterotic string is the 2-form version of $D=10$ supergravity coupled to either $E_{8} \times E_{8}$ or $S O(32)$ super Yang-Mills. Including the Lorentz Chern-Simons term, which follows from stringy corrections [71], the bosonic sector of the action in string $\sigma$-model variables is $[12,13]$

$$
I_{10}(\text { heterotic })=\frac{1}{2 \kappa^{2}} \int d^{10} x \sqrt{-g} e^{-2 \phi}\left(R+4(\partial \phi)^{2}-\frac{1}{2 \cdot 3 !} H^{2}+\frac{\alpha^{\prime}}{8}\left(\operatorname{tr} \hat{R}^{2}-\operatorname{tr} F^{2}\right)\right)
$$

where $\hat{R}$, the curvature generated by the generalized connection

$$
\Omega_{ \pm M}^{A B}=\omega_{M}^{A B} \pm \frac{1}{2} H_{M}^{A B}
$$

where $\omega_{M} A B$ is the usual spin-connection, is explicitly given by

$$
\hat{R}_{J K L}^{I}=R_{J K L}^{I} \mp \frac{1}{2}\left(\nabla_{L} H_{J K}^{I}-\nabla_{K} H_{J L}^{I}\right)+\frac{1}{4}\left(H_{J K}^{M} H_{L M}^{I}-H_{J L}^{M} H_{K M}^{I}\right) .
$$

$d H$ now obeys the Bianchi identity modified by Chern-Simons terms ${ }^{\dagger}$

$$
d H=\frac{\alpha^{\prime}}{4}\left(\operatorname{tr} \hat{R}^{2}-\operatorname{tr} F^{2}\right)
$$

The trace is in the fundamental representation in the case of $S O(32)$ and is defined to be $1 / 30$ times the trace in the adjoint representation in the case of $E_{8} \times E_{8}$.

We begin in section 5.2 with a review of the 't Hooft ansatz for the Yang-Mills instanton [74-77] and then turn to the analogous axionic instanton solution discussed in [78,79]. The generalized curvature of this and all other "fivebrane ansatz" solutions possesses a

$\dagger$ The normalization used below follows that of [72,73], and differs from that of [24] by a factor of 8 . This discrepancy was first brought to our attention by Paul Townsend. 
(anti) self-dual structure similar to that of the 't Hooft ansatz, albeit in the gravitational sector of the string.

In section 5.3 we write down the perturbative "gauge" heterotic soliton solution of [24] and the "symmetric" solution of $[32,33]$, which generalizes the axionic instanton and the gauge solution to a heterotic multi-fivebrane solution with a YM instanton in the gauge sector and an axionic instanton in the gravitational sector in the four-dimensional space transverse to the fivebrane We also discuss the zero modes of these solutions.

In section 5.4 we write down the string soliton solution of [30], which extends the heterotic string soliton solution of chapter 2 to incorporate a Yang-Mills field with eightdimensional instanton structure. In section 5.5 we summarize the octonionic string soliton of [68] and the double-instanton string solution of [67].

\section{2. 't Hooft ansatz and the axionic instanton}

Consider the four-dimensional Euclidean action

$$
S=-\frac{1}{2 g^{2}} \int d^{4} y \operatorname{tr} F_{m n} F^{m n}, \quad m, n=1,2,3,4 .
$$

For gauge group $S U(2)$, the fields may be written as $A_{m}=(g / 2 i) \sigma^{a} A_{m}^{a}$ and $F_{m n}=$ $(g / 2 i) \sigma^{a} F_{m n}^{a}$ (where $\sigma^{a}, a=1,2,3$ are the $2 \times 2$ Pauli matrices). The equation of motion derived from this action is solved by the 't Hooft ansatz [74-77]

$$
A_{m n}=i \bar{\Sigma}_{m n} \partial_{n} \ln f
$$

where $\bar{\Sigma}_{m n}=\bar{\eta}^{i m n}\left(\sigma^{i} / 2\right)$ for $i=1,2,3$, where

$$
\begin{array}{rlrl}
\bar{\eta}^{i m n}=-\bar{\eta}^{i n m} & =\epsilon^{i m n}, & & m, n=1,2,3, \\
& =-\delta^{i m}, & n=4
\end{array}
$$

and where $f^{-1} \square f=0$. The above solution obeys the self-duality condition

$$
F_{m n}=\tilde{F}_{m n}=\frac{1}{2} \epsilon_{m n}^{p q} F_{p q}
$$

The ansatz for the anti-self-dual solution $F_{m n}=-\tilde{F}_{m n}$ is similar, with the $\delta$-term in (5.7) changing sign. To obtain a multi-instanton solution, one solves for $f$ in the four-dimensional space to obtain

$$
f=1+\sum_{i=1}^{k} \frac{\rho_{i}^{2}}{\left|\vec{y}-\vec{a}_{i}\right|^{2}}
$$


where $\rho_{i}$ is the instanton scale size, $\vec{a}_{i}$ the location in four-space of the $i$ th instanton and

$$
k=\frac{1}{16 \pi^{2}} \int_{M^{4}} \operatorname{tr} F^{2}
$$

is the instanton number. Note that this solution has $5 k$ parameters, while the most general (anti) self-dual solution has $8 k$ parameters, or $8 k-3$ if one excludes the 3 zero modes associated with global $S U(2)$ rotations. We do not exclude these modes, however, because as explained below, they belong to the same supermultiplet as the dilatational zero mode.

Now consider the ansatz

$$
\begin{aligned}
g_{m n} & =e^{2 \phi} \delta_{m n} \quad m, n=6,7,8,9, \\
g_{\mu \nu} & =\eta_{\mu \nu} \quad \mu, \nu=0,1,2,3,4,5, \\
H_{m n p} & = \pm 2 \epsilon_{m n p k} \partial^{k} \phi \quad m, n, p, k=6,7,8,9 .
\end{aligned}
$$

Then provided $e^{-2 \phi} \square e^{2 \phi}=0,(5.11)$ is a solution to the low-energy string effective action (3.81) written in terms of string $\sigma$-model variables (where here $F_{3}=H$ ), and which breaks $1 / 2$ the spacetime supersymmetries. In particular, for

$$
e^{2 \phi}=e^{2 \phi_{0}}\left(1+\sum_{i=1}^{N} \frac{\rho_{i}^{2}}{\left|\vec{y}-\vec{a}_{i}\right|^{2}}\right)
$$

we recover the multi-fivebrane solution of section 2.4. The ansatz (5.11) in fact possesses a (anti) self-dual structure in the transverse space (6789), which can be seen by expressing the generalized curvature in covariant form in terms of the dilaton field as [79]

$$
\hat{R}_{j k l}^{i}=\delta_{i l} \nabla_{k} \nabla_{j} \phi-\delta_{i k} \nabla_{l} \nabla_{j} \phi+\delta_{j k} \nabla_{l} \nabla_{i} \phi-\delta_{j l} \nabla_{k} \nabla_{i} \phi \pm \epsilon_{i j k m} \nabla_{l} \nabla_{m} \phi \mp \epsilon_{i j l m} \nabla_{k} \nabla_{m} \phi
$$

It easily follows that [80]

$$
\hat{R}_{j k l}^{i}=\mp \frac{1}{2} \epsilon_{k l}^{m n} \hat{R}_{j m n}^{i}
$$

So the (anti) self-duality appears in the gravitational sector of the string in terms of its generalized curvature thus justifying the name "axionic instanton" for the four-dimensional solution first found in [78]. 


\subsection{The heterotic fivebrane as a soliton: "gauge" versus "symmetric"}

In terms of string $\sigma$-model variables, (2.5) and (2.6) are rewritten as

$$
\begin{gathered}
\delta \lambda=-\frac{1}{2 \sqrt{2}} \Gamma^{M} \partial_{M} \phi \varepsilon+\frac{1}{2 \cdot 2 \sqrt{2} \cdot 3 !} \Gamma^{M N P} H_{M N P} \varepsilon=0, \\
\delta \psi_{M}=\partial_{M} \varepsilon+\frac{1}{4} \Omega_{-M}{ }^{A B} \Gamma_{A B} \varepsilon=0 .
\end{gathered}
$$

For nontrivial Yang-Mills field, the gaugino $\chi$ supersymmetry transformation for zero Fermi fields is given by

$$
\delta \chi=F_{M N} \Gamma^{M N} \varepsilon=0,
$$

which is solved [24] by setting $F_{\mu \nu}=F_{\mu m}=0$ and keeping just an $S U(2)$ subgroup of the gauge group and identifying the corresponding gauge field in the transverse directions with the instanton configuration (5.8) for

$$
\varepsilon=\epsilon_{0} \otimes \eta_{0},
$$

where

$$
\left(1-\gamma_{7}\right) \epsilon_{0}=0, \quad\left(1-\Gamma_{5}\right) \eta_{0}=0 .
$$

This can be seen explicitly from

$$
\begin{aligned}
\delta \chi & =F_{M N} \Gamma^{M N} \varepsilon \\
& =F_{m n} \Sigma_{m n} \varepsilon \\
& =\frac{1}{2} F_{m n} \Sigma^{m n}\left(1-\Gamma_{5}\right) \varepsilon \\
& =0,
\end{aligned}
$$

where we have used the identity $\frac{1}{2} \epsilon_{m n}{ }^{p q} \Sigma_{p q}=-\Gamma_{5} \Sigma_{m n}$. In a similar manner, the equations (5.15) and (5.16) for the ansatz (5.11) and constant chiral spinor (5.18) reduce to

$$
\Omega_{-M}{ }^{A B} \Gamma_{A B} \varepsilon=0,
$$

which is solved precisely because of the self-duality relation

$$
\Omega_{-M}^{m n}=\frac{1}{2} \epsilon^{m n}{ }_{p q} \Omega_{-M}^{p q}
$$

of the generalized connection. The condition (5.19) once again means that one half of the supersymmetries are broken. The generalized connection $\Omega_{-M}{ }^{a b}$ lies in the same $S U(2)$ 
subgroup of $S O(4)$ as $F_{m n}$ does. Note that we have still not specified the dilaton field, which will be determined from the Bianchi identity (5.4).

It is at this stage that we may proceed in one of two directions, depending on whether we consider the pure supergravity theory or whether we include the stringy Lorentz ChernSimons correction. The first route (which we shall shortly justify) leads to the "gauge" solution of [24], while the second route leads to the "symmetric" solution of $[32,33]$. In the first case, using $\operatorname{Tr}_{E_{8}} T^{a} T^{b}$ ( adjoint) $=15 \operatorname{Tr}_{S U(2)} T^{a} T^{b}$ (adjoint) as given in [81] and substituting a single instanton (5.8) with scale size $\rho$, the Bianchi identity (5.4) reduces for the ansatz (5.11) to

$$
\square e^{2 \phi}=-\alpha^{\prime} \frac{24 \rho^{4}}{\left(r^{2}+\rho^{2}\right)^{4}} e^{3 \phi_{0} / 2}
$$

which gives

$$
e^{2 \phi}=e^{2 \phi_{0}}+\alpha^{\prime} \frac{r^{2}+2 \rho^{2}}{\left(r^{2}+\rho^{2}\right)^{2}} e^{3 \phi_{0} / 2}
$$

In analogy with the comparison between the t'Hooft-Polyakov monopole and Dirac's magnetic monopole, the large-distance behavior of the gauge heterotic fivebrane is the same as that of the elementary fivebrane. Therefore, we expect that the Bogomol'nyi bound is also saturated since the mass and the charge depend only on the large distance behavior of the solution. This is indeed the case [24]. For a general instanton solution, the magnetic charge is given by

$$
g_{6}=\frac{1}{\sqrt{2} \kappa} \int_{S^{3}} H=\frac{\sqrt{2} \alpha^{\prime} k \Omega_{3}}{\kappa},
$$

where $k$ is defined in (5.10). Using $\alpha^{\prime}=1 / 2 \pi T_{2}$ and comparing (5.25) with (2.54), we find that the integer in the Dirac quantization rule is given by $n=k$. This implies in particular that the single-instanton solution yields a heterotic fivebrane with a tension equal to that of the fundamental fivebrane.

If one keeps the $\operatorname{tr} \hat{R}^{2}$ term, the so-called "symmetric" solution arises [32,33]. This can be seen by equating the generalized connection $\Omega_{ \pm M}$ to the gauge connection $A_{M}$ [82] so that the corresponding curvature $\hat{R}\left(\Omega_{ \pm}\right)$cancels against the Yang-Mills field strength $F$ and (5.4) reduces to $d H=0$, which then implies $e^{-2 \phi} \square e^{2 \phi}=0$ and (5.13) and (5.14). It then follows that the solution satisfies

$$
F_{p q}^{m n}=\hat{R}_{p q}^{m n}
$$

where both $F$ and $R$ are (anti)self-dual. We shall argue in section 8 that this solution is exact since $A_{M}=\Omega_{ \pm M}$ implies that all the higher order corrections vanish. The solution 
is now given by (5.11) with $e^{2 \phi}=e^{2 \phi_{0}} f$, where $f$ is given by (5.9), but where from the Dirac quantization condition it follows that $\rho_{i}^{2}=e^{-\phi_{0} / 2} n_{i} \alpha^{\prime}$. For this solution, then, the curvature $\hat{R}$ is of the same order in $\alpha^{\prime}$ as $F$ and cannot be dropped in a perturbative approximation. By contrast, had we included the $\operatorname{tr} \hat{R}^{2}$ term when solving for the gauge fivebrane, we would have found that $\operatorname{tr} R^{2}$ vanishes and hence that $\operatorname{tr} \hat{R}^{2}$ is higher order in $\alpha^{\prime}[24]$.

We finally come to the bosonic and fermionic zero modes associated with the above heterotic fivebranes. Since the core of the fivebrane is essentially a four-dimensional YangMills instanton dressed up with axion and dilaton fields, the zero modes that we are going to count are actually those of the instanton. For example, the bosonic (fermionic) zero modes arising from translation (supertranslation) invariance are the same in both the gauge and the gravitational sectors, therefore they need only be counted once. Hence counting the zero modes associated with the instanton is sufficient. There are four translational zero modes (the location of the instanton center) and one dilatational zero mode (the instanton size). Global $S U(2)$ rotations of the instanton add three more zero modes. In addition there are 112 zero modes associated with the minimal embedding of $S U(2)$ in $E_{8}$ or $S O(32)$. These are actually related to the generators of the coset $E_{8} / E_{7} \times S U(2)$ or $S O(32) / S O(28) \times S O(4)$ which do not leave the $S U(2)$ subgroup invariant. So we have a total of 120 bosonic zero modes. The fermionic zero modes can be determined from the Atiyah-Singer index theorem

$$
n_{-}-n_{+}=\frac{1}{8 \pi^{2}} \int \operatorname{Tr} F^{2}
$$

which from (5.10) and $k=1$ gives 60 anti-chiral fermionic zero modes. Since each Weyl spinor in Euclidean four-dimensional space gives 4 off-shell or 2 on-shell degrees of freedom, 60 fermionic zero modes give 240 off-shell or 120 on-shell degrees of freedom. The dynamics of these zero modes can also be described by 60 six-dimensional sympletic Majorana-Weyl spinors transforming covariantly under $S O(1,5)$. This can be achieved through the (super) collective coordinate expansion discussed in some detail in [24,33]. As expected, there are equal on-shell bosonic and fermionic degrees of freedom and the gauge-fixed theory on the worldvolume is given by a $d=6,(2,0)$ supersymmetric $\sigma$-model on a hyperkahler manifold. This suggests that there might exist a Green-Schwarz-like formulation with worldvolume $\kappa$-symmetry for the heterotic fivebrane. For the symmetric solution, since the scale size is quantized in units of $\alpha^{\prime}$, the dilatational zero mode, and all its superpartners, are absent. 


\subsection{Heterotic string solitons}

In the previous section, we saw that the field theory limit of the heterotic string admits a gauge heterotic fivebrane as a soliton. In this section, we show the converse, a result which lends further support to the idea of string/fivebrane duality. After constructing the solution, we examine its zero-modes and suggest that they might correspond to those of the fundamental heterotic string written in a physical gauge.

Our first task is to construct the fivebrane analog of (5.1), i.e. to generalize (2.87) to include the Yang-Mills fields. We claim that the result is

$$
\begin{aligned}
\tilde{I}_{10}(\text { heterotic })=\frac{1}{2 \kappa^{2}} \int d^{10} x & \sqrt{-g} e^{2 \phi / 3}\left(R-\frac{1}{2 \cdot 7 !} K^{2}+\frac{\beta^{\prime}}{24} t^{L M N O P Q R S} \operatorname{tr}_{L M} F_{N O} F_{P Q} F_{R S}\right. \\
& -\frac{1}{8} \operatorname{tr} F_{L M} F_{N O} \operatorname{tr} \hat{R}_{P Q} \hat{R}_{R S}+\frac{1}{32} \operatorname{tr} \hat{R}_{L M} \hat{R}_{N O} \operatorname{tr} \hat{R}_{P Q} \hat{R}_{R S} \\
& \left.\left.+\frac{1}{8} \operatorname{tr} \hat{R}_{L M} \hat{R}_{N O} \hat{R}_{P Q} \hat{R}_{R S}\right)+\cdots\right),
\end{aligned}
$$

where the tensor $t^{I J K L M N P Q}$ is given in [83] by

$$
\begin{aligned}
t^{I J K L M N P Q}= & -\frac{1}{2}\left(g^{I K} g^{J L}-g^{I L} g^{J K}\right)\left(g^{M P} g^{N Q}-g^{M Q} g^{N P}\right) \\
& -\frac{1}{2}\left(g^{K M} g^{L N}-g^{K N} g^{L M}\right)\left(g^{P I} g^{Q J}-g^{P J} g^{Q I}\right) \\
& -\frac{1}{2}\left(g^{I M} g^{J N}-g^{I N} g^{J M}\right)\left(g^{K P} g^{L Q}-g^{K Q} g^{L P}\right) \\
& +\frac{1}{2}\left(g^{J K} g^{L M} g^{P N} g^{Q I}+g^{J M} g^{N K} g^{L P} g^{Q I}\right. \\
& \left.+g^{J M} g^{N P} g^{K Q} g^{L I}+\text { permutations }\right) .
\end{aligned}
$$

$d K$ now obeys the Bianchi identity modified by Chern-Simons corrections

$$
d K=\frac{\beta^{\prime}}{24}\left(\operatorname{tr} F^{4}-\frac{1}{8} \operatorname{tr} F^{2} \operatorname{tr} \hat{R}^{2}+\frac{1}{32}\left(\operatorname{tr} \hat{R}^{2}\right)^{2}+\frac{1}{8} \operatorname{tr} \hat{R}^{4}\right) .
$$

The fivebrane tension $T_{6}$ is given by $1 / \beta^{\prime}=(2 \pi)^{3} T_{6}$. This unconventional quartic action, and the corresponding quartic Chern-Simons terms require some justification. This will necessarily be indirect since, although the super fivebrane [10,9] $\sigma$-model is well-known, the heterotic fivebrane $\sigma$-model has yet to be constructed. Even if we knew it, the quantization of fivebranes is still in its infancy, and it is doubtful that (5.28) could yet be derived as rigorously as (5.1). As in section 2.3, the point of view we adopt is that the fivebrane 
action is obtained by dualizing the string action. In particular, the Bianchi identity for $K$ follows from the field equation for $H$. However, this process does not respect the loop expansion, and what is a tree-level effect in string perturbation theory maybe a oneloop effect in fivebrane perturbation theory and vice-versa. To understand this, we recall the relationship (2.58) between the string loop coupling constant $\mathrm{g}_{2}$, the fivebrane loop coupling constant $\mathrm{g}_{6}$ and $\phi_{0}$. In string variables, each term in the string tree-level action $I_{10}$ (heterotic) is proportional to $e^{-2 \phi}$. Similarly, in fivebrane variables each term in the fivebrane tree-level action $\tilde{I}_{10}$ (heterotic) is proportional to $e^{2 \phi / 3}$. Thus the Green-Schwarz [14] anomaly cancellation term

$$
B \wedge\left(\operatorname{tr} F^{4}-\frac{1}{8} \operatorname{tr} F^{2} \operatorname{tr} \hat{R}^{2}+\frac{1}{32}\left(t r \hat{R}^{2}\right)^{2}+\frac{1}{8} \operatorname{tr} \hat{R}^{4}\right)
$$

which provides a correction to the $H$ field equation, has no $\phi$-dependence in string variables and is therefore seen to be 1-loop in string perturbation theory. Similar remarks apply to the quartic Yang-Mills and gravitational terms which appear in 1-loop corrections to the effective action [84].

On the other hand, both these terms are tree-level in fivebrane perturbation theory, because they both behave like $e^{2 \phi / 3}$ in fivebrane variables. The 1-loop Green-Schwarz corrections to the $H$ field equation now become the tree-level Chern-Simons corrections to the $K$ Bianchi identity (5.30). Similarly, the quartic terms must now be included in the fivebrane tree-level action $\tilde{I}_{10}$ (heterotic). By the same token, the quadratic Yang-Mills term in $I_{10}$ (heterotic) and the Chern-Simons term in (5.4) corresponding to $\left(\operatorname{tr} F^{2}-\operatorname{tr} \hat{R}^{2}\right) \wedge A$ are 1 loop in fivebrane perturbation theory since they are independent of $\phi$ when written in fivebrane variables. We therefore omit them from $\tilde{I}_{10}$ (heterotic). In arriving at (5.28) and (5.30), we have also employed the equation (2.137) with $n=1,2 \kappa^{2}=(2 \pi)^{5} \alpha^{\prime} \beta^{\prime}$ which relates the two fundamental tensions. To further justify (5.28) and (5.30), we note the following. First, $\alpha^{\prime}$ has dimension -2 and $\beta^{\prime}$ has dimension -6 , so purely on dimensional grounds, we would expect a quartic Yang-Mills action. This causes no problems with unitarity. We emphasize that the exact string and fivebrane actions are equivalent; it is merely the division into "classical" plus "quantum" which is different in the two cases. Secondly, the Yang-Mills Chern-Simons corrections (5.30) can be derived directly from the coupling of Yang-Mills fields to a fundamental fivebrane [85], and a case can be made for the gravitational corrections as well $[72,86]$. Thirdly, under the two-parameter rescalings of the background fields discussed in section $2.3, g_{M N} \rightarrow \lambda^{1 / 2} \sigma^{3 / 2} g_{M N}, B_{M N} \rightarrow \lambda^{2} B_{M N}$, 
$A_{M N O P Q R} \rightarrow \sigma^{6} A_{M N O P Q R}, e^{\phi} \rightarrow \lambda^{3} \sigma^{-3} e^{\phi}$, the elementary fivebrane $\sigma$-model action $S_{6}$ and the elementary string $\sigma$-model action $S_{2}$ scale like $S_{6} \rightarrow \sigma^{6} S_{6}$ and $S_{2} \rightarrow \lambda^{2} S_{2}$. In order that $I_{10}$ (heterotic) admits a fivebrane as a soliton [24], it must scale the same way under the $\sigma$ symmetry i.e. $I_{10}$ (heterotic) $\rightarrow \sigma^{6} S I_{10}$ (heterotic). This is indeed the case. Similarly, we are encouraged in our search for a string soliton solution [30] of $\tilde{I}_{10}$ (heterotic) by noting that it scales in the right way under the $\lambda$ symmetry i.e. $\tilde{I}_{10}$ (heterotic) $\rightarrow$ $\lambda^{2} \tilde{I}_{10}$ (heterotic). Without further apology, we now quote the solution which is the analog of the gauge fivebrane rather than the symmetric fivebrane. We therefore ignore the gravitational Chern-Simons terms. The Yang-Mills field is given by the eight-dimensional instanton $[87,88]$

$$
F_{m n}=f(r) \frac{i}{2} \Sigma_{m n}\left(1-\Gamma_{9}\right) / 2, \quad f=4 \rho^{2} /\left(r^{2}+\rho^{2}\right)^{2}
$$

embedded in an $S O(8)$ subgroup of $S O(32)$ or $E_{8}$, where $\rho$ is the instanton size. The instanton winding number is

$$
\tilde{k}=\frac{1}{384 \pi^{4}} \int_{M^{8}} t r F^{4}
$$

Curiously enough, we find $\tilde{k}=1$ in the case of $S O(32)$ but $\tilde{k}=0$ in the case of $E_{8}$ because the group $E_{8}$ has no independent fourth-order Casimir. We refer the reader to $[30,89]$ for further details. The supergravity fields are given by

$$
\begin{aligned}
& d s^{2}=e^{4 \phi / 3} \eta_{\mu \nu} d x^{\mu} d x^{\nu}+e^{-2 \phi / 3} \delta_{m n} d y^{m} d y^{n}, \\
& B_{01}=-e^{2 \phi}
\end{aligned}
$$

and

$$
\begin{array}{rlrl}
e^{-2 \phi} & =1+k_{2} \frac{r^{6}+6 r^{4} \rho^{2}+15 r^{2} \rho^{4}+20 \rho^{6}}{\left(r^{2}+\rho^{2}\right)^{6}}, & \text { for } S O(32) \\
& =1+\frac{|n| k_{2}}{r^{6}}, \quad \text { for } E_{8}
\end{array}
$$

where for convenience we have set $\phi_{0}=0$ and where the constant $k$ is given by $k_{2}=$ $\pi /\left(3 T_{6} \Omega_{7}\right)$ as in (2.132), $n$ is an integer and $\Omega_{7}$ is the volume of the unit seven-sphere. In analogy with the comparison between the t'Hooft-Polyakov monopole and Dirac's magnetic monopole, the large-distance behavior of the gauge heterotic string is the same as that of the elementary string. Therefore, we expect that the Bogomol'nyi bound is also saturated since the mass and the charge depend only on the large distance behavior of the solution. This is indeed the case [30]. This implies in particular that the single-instanton solution yields a gauge heterotic string with a tension equal to that of the fundamental string. We 
emphasize that the solitonic string solution has been established only to tree level in the fivebrane field theory and are a priori valid only for $e^{-2 \phi / 3}<<1$. The question of whether it survives loop corrections remains a topic for future research.

Although we omit the string Chern-Simons terms corresponding to $\left(\operatorname{tr} F^{2}-\operatorname{tr} \hat{R}^{2}\right)$ from our classical fivebrane considerations, they play an important role as the fivebrane analogue of the Green-Schwarz anomaly cancellation terms $[72,86]$. In the case of strings, the combined gravitational and Yang-Mills anomalies for $N=1$ supergravity coupled to a Yang-Mills supermultiplet (with $n$ left-hand Majorana-Weyl spinors in the adjoint representation) can be characterized by a certain 12 -form, $I_{12}$. As discussed in [14], the anomaly can be cancelled only if $I_{12}$ factorizes into an expression of the form $I_{12}=d H \wedge X_{8}$ where $X_{8}$ is an 8 -form. The necessary and sufficient conditions are

$$
\begin{aligned}
n & =\operatorname{dim} G=496, \\
\operatorname{Tr} F^{6} & =\frac{1}{48} \operatorname{Tr} F^{4} \operatorname{Tr} F^{2}-\frac{1}{14,400}\left(\operatorname{Tr} F^{2}\right)^{2} .
\end{aligned}
$$

There are only two solutions for $G: S O(32)$ and $E_{8} \times E_{8}$. The anomaly is then cancelled by the addition of a term in the action $B \wedge X_{8}$. In the case of the fivebrane, we would require that $I_{12}$ factorizes into an expression of the form $I_{12}=X_{4} \wedge d K$ where $X_{4}$ is a 4-form. Assuming that the same $I_{12}$ governs both strings and fivebranes, we discover from [14] that the necessary and sufficient conditions for this to happen are exactly the same as those given in (5.36). Hence we find $S O(32)$ and $E_{8} \times E_{8}$ once more. The anomaly is then cancelled by the term $X_{4} \wedge A[15,16]$. Thus $I_{12}$ takes on the string/fivebrane symmetrical form $I_{12}=d H \wedge d K$.

A different extended soliton string solution, the "octonionic" string, was constructed in [68]. It differs from the gauge heterotic string discussed above by solving $I_{10}$ (heterotic) rather than $\tilde{I}_{10}$ (heterotic). Once again, an eight-dimensional instanton makes its appearance in the transverse space but this time it is the octonionic instanton of $[90,91]$ which preserves $S O(7)$ rather than $S O(8)$. This solution breaks 15/16 of the supersymmetries. Because the Yang-Mills lagrangian is here quadratic rather than quartic, however, the scaling arguments given above for the gauge heterotic string no longer apply. As a consequence, the octonionic string has infinite mass per unit length. 
Another example of a string solution of $I_{10}$ (heterotic) and which therefore also has infinite mass per unit length is the double-instanton string solution of [67]. The ansatz

$$
\begin{aligned}
\phi & =\phi_{1}+\phi_{2}, \\
g_{m n} & =e^{2 \phi_{1}} \delta_{m n} \quad m, n=2,3,4,5, \\
g_{i j} & =e^{2 \phi_{2}} \delta_{i j} \quad i, j=6,7,8,9, \\
g_{\mu \nu} & =\eta_{\mu \nu} \quad \mu, \nu=0,1, \\
H_{m n p} & = \pm 2 \epsilon_{m n p q} \partial^{q} \phi \quad m, n, p, q=2,3,4,5, \\
H_{i j k} & = \pm 2 \epsilon_{i j k l} \partial^{k} \phi \quad i, j, k, l=6,7,8,9
\end{aligned}
$$

with constant chiral spinors $\epsilon_{ \pm}=\epsilon_{2} \otimes \eta_{4} \otimes \eta_{4}^{\prime}$ solves the supersymmetry equations (5.15), (5.16) and (5.17) with zero background fermi fields provided the YM gauge field satisfies the instanton (anti) self-duality condition

$$
\begin{aligned}
F_{m n} & = \pm \frac{1}{2} \epsilon_{m n}^{p q} F_{p q}, \quad m, n, p, q=2,3,4,5 \\
F_{i j} & = \pm \frac{1}{2} \epsilon_{i j}{ }^{k l} F_{k l}, \quad i, j, k, l=6,7,8,9 .
\end{aligned}
$$

The chiralities of the spinors $\epsilon_{2}, \eta_{4}$ and $\eta_{4}^{\prime}$ are correlated by

$$
\left(1 \mp \gamma_{3}\right) \epsilon_{2}=\left(1 \mp \gamma_{5}\right) \eta_{4}=\left(1 \mp \gamma_{5}\right) \eta_{4}^{\prime}=0
$$

so that three-quarters of the spacetime supersymmetries are broken. An exact solution is now obtained as follows. Embed the generalized $\Omega_{ \pm M}$ in an $S U(2) \times S U(2)$ subgroup of the gauge group, and equate it to the gauge connection $A_{M}[82]$ for $M=2,3,4,5,6,7,8,9$ so that $d H=0$ and the corresponding curvature $R\left(\Omega_{ \pm}\right)$cancels against the Yang-Mills field strength $F$ in both subspaces (2345) and (6789). For $e^{-2 \phi_{1}} \square e^{2 \phi_{1}}=e^{-2 \phi_{2}} \square e^{2 \phi_{2}}=0$ it follows that both $F$ and $R$ are (anti) self-dual in both four-dimensional subspaces. The explicit solution for $\phi_{1}$ and $\phi_{2}$ in (5.37) is given by

$$
\begin{aligned}
& e^{2 \phi_{1}}=e^{2 \phi_{1_{0}}}\left(1+\sum_{i=1}^{N} \frac{\rho_{i}^{2}}{\left|\vec{x}-\vec{a}_{i}\right|^{2}}\right), \\
& e^{2 \phi_{2}}=e^{2 \phi_{2_{0}}}\left(1+\sum_{j=1}^{M} \frac{\lambda_{j}^{2}}{\left|\vec{y}-\vec{b}_{j}\right|^{2}}\right),
\end{aligned}
$$

where $\vec{x}$ and $\vec{a}_{i}$ are four-vectors and $\rho_{i}$ instanton scale sizes in the space (2345), and $\vec{y}$ and $\vec{b}_{j}$ are four-vectors and $\lambda_{j}$ instanton scale sizes in the space (6789). Axion charge 
quantization then requires that $\rho_{i}^{2}=e^{-2 \phi_{1_{0}}} n_{i} \alpha^{\prime}$ and $\lambda_{j}^{2}=e^{-2 \phi_{2_{0}}} m_{j} \alpha^{\prime}$, where $n_{i}$ and $m_{j}$ are integers. Note that for $N=0$ or $M=0$ we recover the symmetric fivebrane solution of section 5.2. It is interesting to note that both the charge $Q_{2}=-1 / 2 \int_{S^{7}}{ }^{*} H$ and the mass per unit length $\mathcal{M}_{2}$ of the infinite string diverge. By contrast, all classes of fivebrane solutions have finite charge and mass per unit length as a result of the preservation of half the spacetime supersymmetries and the saturation of a Bogomol'nyi bound. The fact that three-quarters of the spacetime supersymmetries are broken for this solution means that the saturation of the Bogomol'nyi bound is no longer guaranteed, but it is unclear as to whether this would necessarily imply infinite mass per unit length for the string. The divergence of the ADM mass and the topological charge in fact follows from the $1 / r^{2}$ falloff of the fields, and is an infrared phenomenon, as in the case of axion strings in four dimensions. For this reason, the divergence of the energy density and topological charge should not prevent the exsitence of a finite effective action describing this type of string soliton at a scale larger than the core size [68]. It would therefore seem likely that finite mass per unit length analogs of this solution exist, possibly in the context of fundamental fivebrane theory. Another interesting point is that the $D=8$ instanton number $N_{8}$ for this string solution is in general nonzero for gauge group $E_{8} \times E_{8}\left(N_{8}=N M\right.$, where $N$ and $M$ are the $D=4$ instanton numbers in the (2345) and (6789) spaces respectively), since in this case $\left(\operatorname{TrF}^{2}\right)^{2}$ is nonvanishing. This is to be contrasted with the zero $D=8$ instanton number found for the gauge string soliton above. 


\section{String solitons in $\mathrm{D}=4$}

\subsection{String compactification to four dimensions}

It was pointed out in [24] that after toroidal compactification to four dimensions, the fivebrane would appear as either a 0-brane, a 1-brane or a 2-brane, depending on how it wraps around the compactified directions $[70,92,93]$. Thus it ought to be possible to find soliton solutions directly from the four-dimensional string corresponding to monopoles (0-branes), strings (1-branes) and domain walls (2-branes). It is these four-dimensional solitons that form the subject of this section. Such fivebrane-inspired supersymmetric monopoles ${ }^{\dagger}$ were found in $[96,97,98]$ while the string and domain wall solutions were found in [99].

To find these multi-monopole, multi-string and domain wall solutions we shall follow the procedure outlined in [96,97], where it was argued that monopoles solutions of the heterotic string could be obtained by modifying the 't Hooft ansatz for the Yang-Mills instanton. We shall present them from both the $D=10$ and $D=4$ points of view [99]. As with the fivebrane, there are three types of string and domain wall solutions: neutral (i.e. zero Yang-Mills field), gauge and symmetric. In common with the symmetric fivebrane of section 5.3, the symmetric solutions are arguably exact to all orders in $\alpha^{\prime}$, as discussed in section 8 . Of particular interest is the solitonic string, since its couplings to the background fields of supergravity compared to those of the fundamental string are such that the dilaton/axion field $S$ is replaced by the modulus field $T$. It thus belongs to an $O(6,22 ; Z)$ family of dual strings just as there is an $S L(2, Z)$ family of fundamental strings [100]. This accords with the suggestion of $[101,102]$ that string/fivebrane duality interchanges the roles of strong/weak coupling duality and target space duality, which is the subject of section 6.6. Moreover, we shall argue that these $H$-monopoles play the role of winding states for the dual string.

We consider heterotic string theory compactified on a six dimensional torus. The simplest way to derive the low energy effective action for this theory is to start with the

$\dagger$ The bosonic version of the monopole solution we discuss was previously discussed in $[94,95,79]$. 
$N=1$ supergravity theory coupled to $N=1$ super Yang-Mills theory in ten dimensions, and dimensionally reduce the theory from ten to four dimensions [103,104,105,106]. Since at a generic point in the moduli space only the abelian gauge fields give rise to massless fields in four dimensions, it is enough to restrict to the $\mathrm{U}(1)^{16}$ part of the ten-dimensional gauge group. We shall follow the procedure outlined in [107] and confine ourselves to the bosonic sector. The ten-dimensional action is given by,

$$
\begin{array}{r}
I_{10}(\text { string })=\frac{1}{32 \pi} \int d^{10} z \sqrt{-G^{(10)}} e^{-\Phi^{(10)}}\left(R^{(10)}+G^{(10) M N} \partial_{M} \Phi^{(10)} \partial_{N} \Phi^{(10)}\right. \\
\left.-\frac{1}{12} H_{M N P}^{(10)} H^{(10) M N P}-\frac{1}{4} F_{M N}^{(10) I} F^{(10) I M N}\right),
\end{array}
$$

where $G_{M N}^{(10)}, B_{M N}^{(10)}, A_{M}^{(10) I}$, and $\Phi^{(10)}$ are ten-dimensional metric, anti-symmetric tensor field, $U(1)$ gauge fields and the scalar dilaton field respectively $(0 \leq M, N \leq 9,1 \leq I \leq 16)$, and,

$$
\begin{aligned}
F_{M N}^{(10) I} & =\partial_{M} A_{N}^{(10) I}-\partial_{N} A_{M}^{(10) I} \\
H_{M N P}^{(10)} & =\left(\partial_{M} B_{N P}^{(10)}-\frac{1}{2} A_{M}^{(10) I} F_{N P}^{(10) I}\right)+\text { cyclic permutations in } M, N, P .
\end{aligned}
$$

We have set $\kappa^{2}=16 \pi$ for later convenience.

For dimensional reduction, it is convenient to introduce the "four-dimensional fields" $\widehat{G}_{m n}, \widehat{B}_{m n}, \widehat{A}_{m}^{I}, \Phi, A_{\mu}^{(a)}, G_{\mu \nu}$ and $B_{\mu \nu}(1 \leq m \leq 6,0 \leq \mu \leq 3,1 \leq a \leq 28)$ through the relations $[106,108,109]$

$$
\begin{aligned}
& \widehat{G}_{m n}=G_{m+3, n+3}^{(10)}, \quad \widehat{B}_{m n}=B_{m+3, n+3}^{(10)}, \quad \widehat{A}_{m}^{I}=A_{m+3}^{(10) I}, \\
& \frac{1}{2} A_{\mu}^{(m)}=\frac{1}{2} \widehat{G}^{m n} G_{n+3, \mu}^{(10)}, \quad \frac{1}{2} A_{\mu}^{(I+12)}=-\left(\frac{1}{2} A_{\mu}^{(10) I}-\widehat{A}_{n}^{I} A_{\mu}^{(n)}\right), \\
& \frac{1}{2} A_{\mu}^{(m+6)}=\frac{1}{2} B_{(m+3) \mu}^{(10)}-\widehat{B}_{m n} A_{\mu}^{(n)}+\frac{1}{2} \widehat{A}_{m}^{I} A_{\mu}^{(I+12)}, \\
& G_{\mu \nu}=G_{\mu \nu}^{(10)}-G_{(m+3) \mu}^{(10)} G_{(n+3) \nu}^{(10)} \widehat{G}^{m n}, \\
& B_{\mu \nu}=B_{\mu \nu}^{(10)}-\widehat{B}_{m n} A_{\mu}^{(m)} A_{\nu}^{(n)}-\frac{1}{2}\left(A_{\mu}^{(m)} A_{\nu}^{(m+6)}-A_{\nu}^{(m)} A_{\mu}^{(m+6)}\right), \\
& \Phi=\Phi^{(10)}-\frac{1}{2} \ln \operatorname{det} \widehat{G}, \quad 1 \leq m, n \leq 6, \quad 0 \leq \mu, \nu \leq 3, \quad 1 \leq I \leq 16 .
\end{aligned}
$$

Here $\widehat{G}^{m n}$ denotes the inverse of the matrix $\widehat{G}_{m n}$. We now combine the scalar fields $\widehat{G}_{m n}$, $\widehat{B}_{m n}$, and $\widehat{A}_{m}^{I}$ into an $O(6,22)$ matrix valued scalar field $M$. For this we regard $\widehat{G}_{m n}, \widehat{B}_{m n}$ 
and $\widehat{A}_{m}^{I}$ as $6 \times 6,6 \times 6$, and $6 \times 16$ matrices respectively, and $\widehat{C}_{m n}=\frac{1}{2} \widehat{A}_{m}^{I} \widehat{A}_{n}^{I}$ as a $6 \times 6$ matrix, and define $M$ to be the $28 \times 28$ dimensional matrix

$$
M=\left(\begin{array}{ccc}
\widehat{G}^{-1} & \widehat{G}^{-1}(\widehat{B}+\widehat{C}) & \widehat{G}^{-1} \widehat{A} \\
(-\widehat{B}+\widehat{C}) \widehat{G}^{-1} & (\widehat{G}-\widehat{B}+\widehat{C}) \widehat{G}^{-1}(\widehat{G}+\widehat{B}+\widehat{C}) & (\widehat{G}-\widehat{B}+\widehat{C}) \widehat{G}^{-1} \widehat{A} \\
\widehat{A}^{T} \widehat{G}^{-1} & \widehat{A} \widehat{G}^{-1}(\widehat{G}+\widehat{B}+\widehat{C}) & I_{16}+\widehat{A}^{T} \widehat{G}^{-1} \widehat{A}
\end{array}\right)
$$

satisfying

$$
M L M^{T}=L, \quad M^{T}=M, \quad L=\left(\begin{array}{ccc}
0 & I_{6} & 0 \\
I_{6} & 0 & 0 \\
0 & 0 & -I_{16}
\end{array}\right),
$$

where $I_{n}$ denotes the $n \times n$ identity matrix.

The effective action that governs the dynamics of the massless fields in the fourdimensional theory is obtained by substituting the expressions for the ten-dimensional fields in terms of the four-dimensional fields in (6.1), and taking all field configurations to be independent of the internal coordinates. The result is

$$
\begin{gathered}
I_{4}(\text { string })=\frac{1}{32 \pi} \int d^{4} x \sqrt{-G} e^{-\Phi}\left[R_{G}+G^{\mu \nu} \partial_{\mu} \Phi \partial_{\nu} \Phi-\frac{1}{12} G^{\mu \mu^{\prime}} G^{\nu \nu^{\prime}} G^{\rho \rho^{\prime}} H_{\mu \nu \rho} H_{\mu^{\prime} \nu^{\prime} \rho^{\prime}}\right. \\
\left.-\frac{1}{4} G^{\mu \mu^{\prime}} G^{\nu \nu^{\prime}} F_{\mu \nu}^{(a)}(L M L)_{a b} F_{\mu^{\prime} \nu^{\prime}}^{(b)}+\frac{1}{8} G^{\mu \nu} \operatorname{Tr}\left(\partial_{\mu} M L \partial_{\nu} M L\right)\right],
\end{gathered}
$$

where

$$
\begin{aligned}
F_{\mu \nu}^{(a)} & =\partial_{\mu} A_{\nu}^{(a)}-\partial_{\nu} A_{\mu}^{(a)}, \\
H_{\mu \nu \rho} & =\left(\partial_{\mu} B_{\nu \rho}+2 A_{\mu}^{(a)} L_{a b} F_{\nu \rho}^{(b)}\right)+\text { cyclic permutations of } \mu, \nu, \rho,
\end{aligned}
$$

and $R_{G}$ is the scalar curvature associated with the four-dimensional metric $G_{\mu \nu}$. In deriving this result we have taken $\int d^{6} y=1$, where $y^{m}(1 \leq m \leq 6)$ denote the coordinates labeling the six-dimensional torus. Here $\Phi$ is the $D=4$ dilaton, $R_{G}$ is the scalar curvature formed from the string metric $G_{\mu \nu}$, related to the canonical metric $g_{\mu \nu}$ by $G_{\mu \nu} \equiv e^{\Phi} g_{\mu \nu}$. $B_{\mu \nu}$ is the 2 -form which couples to the string worldsheet and $A_{\mu}{ }^{a}(a=1, \ldots, 28)$ are the abelian gauge fields. $M$ is a symmetric $28 \times 28$ dimensional matrix of scalar fields satisfying $M L M=L$ where $L$ is the invariant metric on $O(6,22)$ :

$$
L=\left(\begin{array}{ccc}
0 & I_{6} & 0 \\
I_{6} & 0 & 0 \\
0 & 0 & -I_{16}
\end{array}\right)
$$

\section{2. $T$ duality and $S$ duality}

The action is invariant under the $O(6,22)$ transformations $M \rightarrow \Omega M \Omega^{T}, A_{\mu}{ }^{a} \rightarrow$ $\Omega^{a}{ }_{b} A_{\mu}{ }^{b}, G_{\mu \nu} \rightarrow G_{\mu \nu}, B_{\mu \nu} \rightarrow B_{\mu \nu}, \Phi \rightarrow \Phi$, where $\Omega$ is an $O(6,22)$ matrix satisfying $\Omega^{T} L \Omega=L$. $T$-duality corresponds to the $O(6,22 ; Z)$ subgroup and is known 
to be an exact symmetry of the full string theory [110]. The equations of motion, though not the action, are also invariant under the $S L(2, R)$ transformations: $\mathcal{M} \rightarrow$ $\omega \mathcal{M} \omega^{T}, \mathcal{F}_{\mu \nu}{ }^{a \alpha} \rightarrow \omega^{\alpha}{ }_{\beta} \mathcal{F}_{\mu \nu}{ }^{a \beta}, g_{\mu \nu} \rightarrow g_{\mu \nu}, M \rightarrow M$ where $\alpha=1,2$ with $\mathcal{F}_{\mu \nu}{ }^{a 1}=F_{\mu \nu}{ }^{a}$ and $\mathcal{F}_{\mu \nu}{ }^{a 2}=\left(\lambda_{2}(M L)^{a}{ }_{b} \tilde{F}_{\mu \nu}{ }^{b}+\lambda_{1} F_{\mu \nu}{ }^{a}\right)$, where $\omega$ is an $S L(2, R)$ matrix satisfying $\omega^{T} \mathcal{L} \omega=\mathcal{L}$ and where

$$
\mathcal{M}=\frac{1}{\lambda_{2}}\left(\begin{array}{cc}
1 & \lambda_{1} \\
\lambda_{1} & |\lambda|^{2}
\end{array}\right), \mathcal{L}=\left(\begin{array}{cc}
0 & 1 \\
-1 & 0
\end{array}\right)
$$

$\lambda$ is given by $\lambda=\Psi+i e^{-\Phi} \equiv \lambda_{1}+i \lambda_{2}$. The axion $\Psi$ is defined through the relation $\sqrt{-g} H^{\mu \nu \rho}=-e^{2 \Phi} \epsilon^{\mu \nu \rho \sigma} \partial_{\sigma} \Psi$. S-duality corresponds to the $S L(2, Z)$ subgroup and there is now a good deal of evidence $[111,112,108,113,107,114,115,109,99,116]$ in favor of its also being an exact symmetry of the full string theory. See also [117]. For the restricted class of configurations obtained by setting to zero the 16 gauge fields $F^{13 \rightarrow 28}$ originating from the ten-dimensional gauge fields, it is possible to define a dual action [109] which has manifest $S L(2, R)$ symmetry. The field strengths $F^{1 \rightarrow 6}$, whose origin resides in the $D=10$ metric, remain the same but the $F^{7 \rightarrow 12}$, whose origin resides in the $D=102$-form, are replaced by their duals. The equations of motion are also invariant under $O(6,6)$; the action is not except for the $S L(6, R)$ subgroup which acts trivially. This action is precisely the one obtained by dimensional reduction from the dual (6-form) version of $D=10$ supergravity which couples to the worldvolume of the fivebrane $[17,24]$ and for which the axion is just the 6 -form component lying in the extra 6 dimensions.

\subsection{Fivebrane compactification to four dimensions}

Once again we shall follow the procedure of [107]. For metric $\widetilde{G}_{M N}^{(10)}$, a six-form field $\widetilde{B}_{M_{1} \ldots M_{6}}^{(10)}$, and dilaton field $\widetilde{\Phi}^{(10)}$, we can rewrite $(2.87)$ as

$$
\begin{aligned}
I_{10}(\text { fivebrane })=\frac{1}{32 \pi} \int d^{10} z \sqrt{-\widetilde{G}^{(10)}} e^{\widetilde{\Phi}^{(10)} / 3}\left(\widetilde{R}^{(10)}\right. & \\
& \left.\quad-\frac{1}{2 \times 7 !} \widetilde{G}^{(10) M_{1} N_{1}} \ldots \widetilde{G}^{(10) M_{7} N_{7}} \widetilde{H}_{M_{1} \ldots M_{7}}^{(10)} \widetilde{H}_{N_{1} \ldots N_{7}}^{(10)}\right),
\end{aligned}
$$

where

$$
\widetilde{H}_{M_{1} \ldots M_{7}}^{(10)}=\partial_{\left[M_{1}\right.} \widetilde{B}_{\left.M_{2} \ldots M_{7}\right]}^{(10)} .
$$


We showed in section 2 that the equations of motion and the Bianchi identities derived from this action can be shown to be identical to those derived from the action (6.1) provided we make the identifications

$$
\begin{aligned}
& \widetilde{\Phi}^{(10)}=\Phi^{(10)}, \quad \widetilde{G}_{M N}^{(10)}=e^{-\Phi^{(10)} / 3} G_{M N}^{(10)}, \\
& \sqrt{-\widetilde{G}^{(10)}} e^{\widetilde{\Phi}^{(10)} / 3} \widetilde{G}^{(10) M_{1} N_{1}} \ldots \widetilde{G}^{(10) M_{7} N_{7}} \widetilde{H}_{N_{1} \ldots N_{7}}^{(10)}=-\frac{1}{3 !} \epsilon^{M_{1} \ldots M_{10}} H_{M_{8} M_{9} M_{10}} .
\end{aligned}
$$

In order to carry out the dimensional reduction of this theory from ten to four dimensions, it is convenient to introduce the "four-dimensional fields" $\lambda, \mathcal{C}_{\mu}^{m}, \mathcal{D}_{\mu}^{m}, \widehat{G}_{m n}, \mathcal{B}_{\mu \nu}^{m n}$, $\mathcal{E}_{\mu \nu \rho}^{m n p}$ and $g_{\mu \nu}$ through the relations [101]:

$$
\begin{aligned}
\widehat{G}_{m n}= & e^{\widetilde{\Phi}^{(10)} / 3} \widetilde{G}_{m+3, n+3}^{(10)}, \quad \lambda_{1}=\frac{1}{6 !} \widetilde{B}_{m_{1}+3, \ldots m_{6}+3}^{(10)} \epsilon^{m_{1} \ldots m_{6}}, \quad \lambda_{2}=\sqrt{\operatorname{det} \widehat{G}} e^{-\widetilde{\Phi}^{(10)},}, \\
\mathcal{C}_{\mu}^{m}= & e^{\widetilde{\Phi}^{(10)} / 3} \widehat{G}^{m n} \widetilde{G}_{(n+3) \mu}^{(10)}, \quad \mathcal{D}_{\mu}^{m_{1}}=\frac{1}{5 !} \epsilon^{m_{1} \ldots m_{6}} \widetilde{B}_{\mu\left(m_{2}+3\right) \ldots\left(m_{6}+3\right)}^{(10)}-\lambda_{1} \mathcal{C}_{\mu}^{m_{1}} \\
\mathcal{B}_{\mu \nu}^{m_{1} m_{2}}= & \frac{1}{4 !} \epsilon^{m_{1} \ldots m_{6}} \widetilde{B}_{\mu \nu\left(m_{3}+3\right) \ldots\left(m_{6}+3\right)}^{(10)} \\
& -\left[\left(\lambda_{1} \mathcal{C}_{\mu}^{m_{1}} \mathcal{C}_{\nu}^{m_{2}}+\frac{1}{2} \mathcal{D}_{\mu}^{m_{1}} \mathcal{C}_{\nu}^{m_{2}}-\frac{1}{2} \mathcal{D}_{\nu}^{m_{1}} \mathcal{C}_{\mu}^{m_{2}}\right)-\left(m_{1} \leftrightarrow m_{2}\right)\right] \\
\mathcal{E}_{\mu \nu \rho}^{m_{1} m_{2} m_{3}}= & \frac{1}{3 !} \epsilon^{m_{1} \ldots m_{6}} \widetilde{B}_{\mu \nu \rho\left(m_{4}+3\right) \ldots\left(m_{6}+3\right)}^{(10)}, \\
g_{\mu \nu}= & \left(\lambda_{2}\right)^{2 / 3}(\operatorname{det} \widehat{G})^{\frac{1}{6}}\left(\widetilde{G}_{\mu \nu}^{(10)}-\widetilde{G}_{(m+3)(n+3)}^{(10)} \mathcal{C}_{\mu}^{m} \mathcal{C}_{\nu}^{n}\right),
\end{aligned}
$$

and the corresponding field strengths,

$$
\begin{aligned}
F_{\mu \nu}^{(\mathcal{C}) m}= & \partial_{\mu} \mathcal{C}_{\nu}^{m}-\partial_{\nu} \mathcal{C}_{\mu}^{m}, \quad F_{\mu \nu}^{(\mathcal{D}) m}=\partial_{\mu} \mathcal{D}_{\nu}^{m}-\partial_{\nu} \mathcal{D}_{\mu}^{m} \\
K_{\mu \nu \rho}^{m n}= & \left(\left[\partial_{\mu} \mathcal{B}_{\nu \rho}^{m n}-\frac{1}{2}\left\{\left(\mathcal{C}_{\rho}^{n} F_{\mu \nu}^{(\mathcal{D}) m}+\mathcal{D}_{\rho}^{n} F_{\mu \nu}^{(\mathcal{C}) m}\right)-(m \leftrightarrow n)\right\}\right]\right. \\
& + \text { cyclic permutations of } \mu, \nu, \rho) \\
\mathcal{K}_{\mu \nu \rho \sigma}^{m n n}= & {\left[\partial_{\mu} \mathcal{E}_{\nu \rho \sigma}^{m n p}+(-1)^{P} \cdot \text { cyclic permutations of } \mu, \nu, \rho, \sigma\right] } \\
& -\left[\left(\mathcal{C}_{\sigma}^{p} K_{\mu \nu \rho}^{m n}+\text { cyclic permutations of } m, n, p\right)\right. \\
& \left.+(-1)^{P} \cdot \text { cyclic permutations of } \mu, \nu, \rho, \sigma\right] \\
& -\left[\left\{\mathcal{C}_{\sigma}^{p} \mathcal{C}_{\rho}^{n}\left(F_{\mu \nu}^{(\mathcal{D}) m}+\lambda_{1} F_{\mu \nu}^{(\mathcal{C}) m}\right)+(-1)^{P} \cdot \text { all permutations of } m, n, p\right\}\right. \\
& \left.+(-1)^{P} \cdot \text { inequivalent permutations of } \mu, \nu, \rho, \sigma\right] \\
& -\left[\left(\mathcal{C}_{\sigma}^{p} \mathcal{C}_{\rho}^{n} \mathcal{C}_{\nu}^{m} \partial_{\mu} \lambda_{1}+(-1)^{P} \cdot \text { all permutations of } m, n, p\right)\right. \\
& \left.+(-1)^{P} \cdot \text { cyclic permutations of } \mu, \nu, \rho, \sigma\right] .
\end{aligned}
$$


Using the relationship between the fields in the two formulations of the ten-dimensional $N=1$ supergravity theory given in (6.12), and the definition of the fields $\lambda_{1}, \lambda_{2}, \widehat{G}_{m n}$ and $g_{\mu \nu}$ in the two formulations, one can easily verify that the two sets of definitions lead to identical $\lambda, \widehat{G}_{m n}$ and $g_{\mu \nu}$.

The action (6.10), expressed in terms of these "four-dimensional fields", is given by,

$$
\begin{aligned}
& S=\frac{1}{32 \pi} \int d^{4} x \sqrt{-g}\left[R-\frac{1}{2\left(\lambda_{2}\right)^{2}} g^{\mu \nu} \partial_{\mu} \bar{\lambda} \partial_{\nu} \lambda+\frac{1}{4} g^{\mu \nu} \operatorname{Tr}\left(\partial_{\mu} \widehat{G} \partial_{\nu} \widehat{G}^{-1}\right)\right. \\
& -\frac{1}{4} \widehat{G}_{m n} g^{\mu \rho} g^{\nu \sigma}\left(\begin{array}{ll}
F_{\mu \nu}^{(\mathcal{C}) m} & -F_{\mu \nu}^{(\mathcal{D}) m}
\end{array}\right) \mathcal{L}^{T} \mathcal{M L}\left(\begin{array}{c}
F_{\rho \sigma}^{(\mathcal{C}) n} \\
-F_{\rho \sigma}^{(\mathcal{D}) n}
\end{array}\right) \\
& -\frac{1}{2 \times 2 ! \times 3 !} \widehat{G}_{m_{1} n_{1}} \widehat{G}_{m_{2} n_{2}} g^{\mu_{1} \nu_{1}} \cdots g^{\mu_{3} \nu_{3}} K_{\mu_{1} \mu_{2} \mu_{3}}^{m_{1} m_{2}} K_{\nu_{1} \nu_{2} \nu_{3}}^{n_{1} n_{2}} \\
& \left.-\frac{\lambda_{2}}{2 \times 3 ! \times 4 !} \widehat{G}_{m_{1} n_{1}} \cdots \widehat{G}_{m_{3} n_{3}} g^{\mu_{1} \nu_{1}} \cdots g^{\mu_{4} \nu_{4}} \mathcal{K}_{\mu_{1} \ldots \mu_{4}}^{m_{1} \ldots m_{3}} \mathcal{K}_{\nu_{1} \ldots \nu_{4}}^{n_{1} \ldots n_{3}}\right] \text {, }
\end{aligned}
$$

where $\mathcal{M}$ has been defined in (6.9), and Tr denotes trace over the indices $m, n(1 \leq m, n \leq$ $6)$. The equation of motion for $\mathcal{E}_{\mu_{1} \mu_{2} \mu_{3}}^{m_{1} m_{2} m_{3}}$ gives

$$
\partial_{\nu_{1}}\left[\lambda_{2} \sqrt{-g} \widehat{G}_{m_{1} n_{1}} \ldots \widehat{G}_{m_{3} n_{3}} g^{\mu_{1} \nu_{1}} \ldots g^{\mu_{4} \nu_{4}} \mathcal{K}_{\nu_{1} \ldots \nu_{4}}^{n_{1} \ldots n_{3}}\right]=0 .
$$

Since $\mathcal{K}_{\nu_{1} \ldots \nu_{4}}^{n_{1} \ldots n_{3}}$ is antisymmetric in $\nu_{1}, \ldots \nu_{4}$, we may write

$$
\lambda_{2} \sqrt{-g} \widehat{G}_{m_{1} n_{1}} \ldots \widehat{G}_{m_{3} n_{3}} g^{\mu_{1} \nu_{1}} \ldots g^{\mu_{4} \nu_{4}} \mathcal{K}_{\nu_{1} \ldots \nu_{4}}^{n_{1} \ldots n_{3}}=\epsilon^{\mu_{1} \ldots \mu_{4}} H_{m_{1} m_{2} m_{3}}
$$

for some $H_{m n p}$. The equation (6.16) then takes the form:

$$
\partial_{\nu} H_{m_{1} m_{2} m_{3}}=0,
$$

showing that $H_{m n p}$ is a constant. Comparison with the original formulation of the theory shows that the $H_{m n p}$ are proportional to the internal components of the three form field strength $H_{M N P}^{(10)}$. During the dimensional reduction of the original ten-dimensional $N=1$ supergravity theory, we had set these constants to zero. Hence, if we want to recover the same theory, we must set them to zero here too. This gives

$$
\mathcal{K}_{\mu_{1} \ldots \mu_{4}}^{m_{1} \ldots m_{3}}=0
$$

The action (6.15) now reduces to

$$
\begin{aligned}
& S=\frac{1}{32 \pi} \int d^{4} x \sqrt{-g}\left[R-\frac{1}{4} g^{\mu \nu} \operatorname{tr}\left(\partial_{\mu} \mathcal{M L} \partial_{\nu} \mathcal{M L}\right)+\frac{1}{4} g^{\mu \nu} \operatorname{Tr}\left(\partial_{\mu} \widehat{G} \partial_{\nu} \widehat{G}^{-1}\right)\right. \\
& -\frac{1}{4} \widehat{G}_{m n} g^{\mu \rho} g^{\nu \sigma}\left(\begin{array}{ll}
F_{\mu \nu}^{(\mathcal{C}) m} & -F_{\mu \nu}^{(\mathcal{D}) m}
\end{array}\right) \mathcal{L}^{T} \mathcal{M L}\left(\begin{array}{c}
F_{\rho \sigma}^{(\mathcal{C}) n} \\
-F_{\rho \sigma}^{(\mathcal{D}) n}
\end{array}\right) \\
& \left.-\frac{1}{2 \times 2 ! \times 3 !} \widehat{G}_{m_{1} n_{1}} \widehat{G}_{m_{2} n_{2}} g^{\mu_{1} \nu_{1}} \cdots g^{\mu_{3} \nu_{3}} K_{\mu_{1} \mu_{2} \mu_{3}}^{m_{1} m_{2}} K_{\nu_{1} \nu_{2} \nu_{3}}^{n_{1} n_{2}}\right],
\end{aligned}
$$


and has manifest $\mathrm{SL}(2, \mathrm{R})$ invariance

$$
\mathcal{M} \rightarrow \omega \mathcal{M} \omega^{T}, \quad\left(\begin{array}{c}
\mathcal{C}_{\mu}^{m} \\
-\mathcal{D}_{\mu}^{m}
\end{array}\right) \rightarrow \omega\left(\begin{array}{c}
\mathcal{C}_{\mu}^{m} \\
-\mathcal{D}_{\mu}^{m}
\end{array}\right)
$$

with all other fields remaining invariant under the $\mathrm{SL}(2, \mathrm{R})$ transformation.

This shows that the $S L(2, R)$ symmetry arises naturally in the four-dimensional theory obtained from the dimensional reduction of the dual formulation of the $N=1$ supergravity theory in ten dimensions, just as the $O(6,6)$ or $O(6,22)$ symmetry arises naturally in the dimensional reduction of the usual $N=1$ supergravity theory from ten to four dimensions. This result provides another reason for believing that the roles of $S$ and $T$ duality are interchanged in going from string to fivebrane, and is entirely consistent with an earlier observation that the dual theory interchanges the worldsheet and spacetime loop expansions [31]. In this light, the need to treat the above 16 gauge fields on a different footing is only to be expected since in the dual formulation their kinetic terms are 1-loop effects.

\subsection{Montonen-Olive revisited}

Following [111,112] (see also [117]), and generalizing an earlier idea of Montonen and Olive $[42,37,43]$, Schwarz and Sen have conjectured [115,107] on the basis of string/fivebrane duality that, when the solitonic excitations are included, the full string spectrum is invariant not only under the target space $O(6,22 ; Z)$ ( $T$-duality) but also under the strong/weak coupling $S L(2, Z)$ ( $S$-duality). They have constructed a manifestly $S$ and $T$ duality invariant mass spectrum. $T$-duality transforms electrically charged winding states into electrically charged Kaluza-Klein states, but $S$-duality transforms elementary electrically charged string states into solitonic monopole and dyon states.

We now turn to the electric and magnetic charge spectrum. Schwarz and Sen $[115,107]$ present an $O(6,22 ; Z)$ and $S L(2, Z)$ invariant expression for the mass of particles saturating the strong Bogomol'nyi bound $m=\left|Z_{1}\right|=\left|Z_{2}\right|$ :

$$
m^{2}=\frac{1}{16}\left(\begin{array}{ll}
\alpha^{a} & \beta^{a}
\end{array}\right) \mathcal{M}^{0}\left(M^{0}+L\right)_{a b}\left(\begin{array}{c}
\alpha^{b} \\
\beta^{b}
\end{array}\right),
$$

where a superscript 0 denotes the constant asymptotic values of the fields. Here $\alpha^{a}$ and $\beta^{a}(a=1, \ldots, 28)$ each belong to an even self-dual Lorentzian lattice $\Lambda$ with metric given 
by $L$ and are related to the electric and magnetic charge vectors $\left(Q^{a}, P^{a}\right)$ by $\left(Q^{a}, P^{a}\right)=$ $\left(M_{a b}{ }^{0}\left(\alpha^{b}+\lambda_{1}^{0} \beta^{b}\right) / \lambda_{2}{ }^{0}, L_{a b} \beta^{b}\right)$.

Eq. (6.22) suggests that it is natural to combine the vectors $\alpha^{a}$ and $\beta^{a}$ into a single 56-dimensional vector $\xi=\left(\alpha^{a}, \beta^{a}\right)$ which now belongs to a 56 -dimensional lattice $\Gamma$. The new lattice $\Gamma$ is self-dual not only with respect to the metric $\hat{\mathcal{L}}=\mathcal{L} \otimes L$. The latter condition says that, for any two vectors $\xi=\left(\alpha^{a}, \beta^{a}\right)$ and $\xi^{\prime}=\left(\alpha^{\prime a}, \beta^{\prime a}\right)$ belonging to the lattice $\Gamma$

$$
\xi^{T} \hat{\mathcal{L}} \eta^{\prime}=\alpha^{a} L_{a b} \beta^{\prime b}-\alpha^{\prime a} L_{a b} \beta^{b}=\text { integer. }
$$

This is just the Dirac-Schwinger-Zwanziger-Witten quantization condition for the magnetic charge $[63,65,64,66]$. We shall return to this Sen-Schwarz spectrum in section 6.8.

\subsection{Monopoles, strings and domain walls}

In this section we now turn to the explicit solutions for the monopole, string and domain wall obtained by wrapping the fivebrane around the extra dimensions. We shall see that the neutral monopoles may be identified with the monopole states in the SenSchwarz spectrum. Moreover, we shall argue that they are the winding states of the dual string soliton regarded as a fundamental theory in its own right. From the 't Hooft ansatz for the Yang-Mills instanton (see section 5), depending on how many of the four coordinates $f$ is allowed to depend and depending on whether we compactify, we shall obtain $D=10$ multi-fivebrane and $D=4$ multi-monopole, multi-string and domain wall solutions. This follows from the observation that the arguments of chapter 5 do not depend on the precise form of $f$ or the dilaton function [99].

We rewrite the ansatz (5.11) with the following notation

$$
\begin{aligned}
g_{\mu \nu} & =e^{2 \phi} \delta_{m n} \quad m, n=1,2,3,4, \\
g_{\mu \nu} & =\eta_{\mu \nu} \quad \mu, \nu=0,5,6,7,8,9, \\
H_{m n p} & = \pm 2 \epsilon_{m n p k} \partial^{k} \phi \quad m, n, p, k=1,2,3,4,
\end{aligned}
$$

with $e^{-2 \phi} \square e^{2 \phi}=0$. Let us single out a direction in the transverse four-space (say $x^{4}$ ) and assume all fields are independent of this coordinate. Since all fields are already independent of $x^{5}, x^{6}, x^{7}, x^{8}, x^{9}$, we may consistently assume the $x^{4}, x^{5}, x^{6}, x^{7}, x^{8}, x^{9}$ are compactified on a six-dimensional torus, where we shall take the $x^{4}$ circle to have circumference $2 \pi R$ 
and the rest to have circumference $2 \pi \sqrt{\alpha^{\prime}}$, so that $(2 \pi)^{6} R_{1} \alpha^{\prime 5 / 2} \kappa_{4}^{2}=\kappa_{10}^{2}$. Going back to the 't Hooft ansatz (5.6), the solution for $f$ satisfying the $f^{-1} \square f=0$ has the form

$$
f_{M}=1+\sum_{i=1}^{N} \frac{m_{i}}{\left|\vec{x}-\vec{a}_{i}\right|}
$$

where $m_{i}$ is proportional to the charge and $\vec{a}_{i}$ the location in the three-space (123) of the $i$ th instanton string. If we make the identification $\Phi \equiv A_{4}$ then the lagrangian density may be rewritten as

$$
F_{\mu \nu}^{a} F_{\mu \nu}^{a}=F_{j k}^{a} F_{j k}^{a}+2 F_{k 4}^{a} F_{k 4}^{a}=F_{j k}^{a} F_{j k}^{a}+2 D_{k} \Phi^{a} D_{k} \Phi^{a}
$$

where $j, k=1,2,3$. We now go to $3+1$ space (0123) with the Lagrangian density

$$
\mathcal{L}=-\frac{1}{4} G_{\alpha \beta}^{a} G^{\alpha \beta a}-\frac{1}{2} D_{\alpha} \Phi^{a} D^{\alpha} \Phi^{a}
$$

where $\alpha, \beta=0,1,2,3$. It follows that the above ansatz is a static solution with $A_{0}^{a}=0$ and all time derivatives vanish. The solution in $3+1$ dimensions has the form

$$
\begin{aligned}
\Phi^{a} & =\mp \frac{1}{g} \delta^{a j} \partial_{j} \ln f_{M}, \\
A_{k}^{a} & =\frac{1}{g} \epsilon^{a k j} \partial_{j} \ln f_{M},
\end{aligned}
$$

where $j, k=1,2,3$ and $g$ is the YM coupling constant. This solution represents a multiinstanton string configuration with sources at $\vec{a}_{i}, i=1,2 \ldots N[96,97]$. ${ }^{*}$ For $e^{2 \phi}=e^{2 \phi_{0}} f_{M}$, we obtain a neutral solution and a symmetric solution [96,97] respectively depending on whether we set the gauge field equal to zero or to the generalized connection. In both cases, the magnetic charge is given by $\tilde{g}_{1}=\sqrt{2} \kappa_{4} \widetilde{T_{1}}$, where $\widetilde{T_{1}}=\widetilde{T_{6}}\left(2 \pi \sqrt{\alpha^{\prime}}\right)^{5}$ obeys, from (2.137), the quantization condition

$$
2 \pi R_{1} \kappa_{4}^{2} T_{2} \widetilde{T_{1}}=n \pi
$$

This implies $m_{i}=n_{i} \alpha^{\prime} / 2 R_{1}$. Similarly the "electric" charge of the fundamental string is $e_{1}=\sqrt{2} \kappa_{4} T_{1}$, where $T_{1}=T_{2} 2 \pi R_{1}$, and hence

$$
e_{1} \tilde{g}_{1}=2 \pi n
$$

* This modified 't Hooft ansatz does not represent a true multi-monopole solution of the pure Yang-Mills field theory but nevertheless possesses some properties analogous to those of a multi-monopole solution $[118,96,97,98]$. 
as expected. A noteworthy feature of this solution is that the divergences from both gauge and gravitational sectors cancel in the classical action.

It is straightforward to reduce the above solution to an explicit solution in the fourdimensional space (0123). The gauge field reduction is exactly as above, i.e. we replace $A_{4}$ with the scalar field $\Phi$. In the gravitational sector, the reduction from ten to five dimensions is trivial, as the metric is flat in the subspace (56789). In going from five to four dimensions, one follows the usual Kaluza-Klein procedure of replacing $g_{44}$ with a scalar field $e^{-2 \sigma_{1}}$. The tree-level effective action reduces in four dimensions to

$$
I_{4}=\frac{1}{2 \kappa_{4}^{2}} \int d^{4} x \sqrt{-g} e^{-2 \phi-\sigma_{1}}\left(R+4(\partial \phi)^{2}+4 \partial \sigma \cdot \partial \phi-e^{2 \sigma_{1}} \frac{M_{\alpha \beta} M^{\alpha \beta}}{4}\right),
$$

where $\alpha, \beta=0,1,2,3$ and where $M_{\alpha \beta}=H_{\alpha \beta 4}=\partial_{\alpha} B_{\beta 4}-\partial_{\beta} B_{\alpha 4}$. The four-dimensional monopole solution for this reduced action is then given by

$$
\begin{aligned}
e^{2 \phi}=e^{-2 \sigma_{1}} & =e^{2 \phi_{0}}\left(1+\sum_{i=1}^{N} \frac{m_{i}}{\left|\vec{x}-\vec{a}_{i}\right|}\right), \\
d s^{2} & =-d t^{2}+e^{2 \phi}\left(d x_{1}^{2}+d x_{2}^{2}+d x_{3}^{2}\right), \\
M_{i j} & = \pm \epsilon_{i j k} \partial_{k} e^{2 \phi}, \quad i, j, k=1,2,3 .
\end{aligned}
$$

For a single monopole, in particular, we have

$$
M_{\theta \phi}= \pm m \sin \theta
$$

which is the magnetic field strength of a Dirac monopole. Note, however, that this monopole did not arise from the Yang-Mills field strength $F_{M N}$ but from the compactified three-form $H$, and arises in all versions of this solution. In particular, one may obtain a multi-magnetic monopole solution of purely bosonic string theory [79].

We now modify the solution of the 't Hooft ansatz even further and choose two directions in the four-space (1234) (say $x^{3}$ and $x^{4}$ ) and assume all fields are independent of both of these coordinates. We may now consistently assume that $x^{3}, x^{4}, x^{6}, x^{7}, x^{8}, x^{9}$ are compactified on a six-dimensional torus, where we shall take the $x^{3}$ and $x^{4}$ circles to have circumference $2 \pi R_{2}$ and the remainder to have circumference $2 \pi \sqrt{\alpha^{\prime}}$, so that $(2 \pi)^{6} R_{2}^{2} \alpha^{\prime 2} \kappa_{4}^{2}=\kappa_{10}^{2}$. Then the solution for $f$ satisfying $f^{-1} \square f=0$ has multi-string structure

$$
f_{S}=1-\sum_{i=1}^{N} \lambda_{i} \ln \left|\vec{x}-\vec{a}_{i}\right|,
$$


where $\lambda_{i}$ is the charge per unit length and $\vec{a}_{i}$ the location in the two-space (12) of the $i$ th string. If we make the identification $\Phi \equiv A_{4}$ and $\Psi \equiv A_{3}$ then the lagrangian density for the above ansatz can be rewritten as

$$
F_{\mu \nu}^{a} F_{\mu \nu}^{a}=F_{j k}^{a} F_{j k}^{a}+2 D_{k} \Phi^{a} D_{k} \Phi^{a}+2 D_{k} \Psi^{a} D_{k} \Psi^{a}
$$

where $j, k=1,2$. We now go to the $3+1$ space (0125) with the lagrangian density

$$
\mathcal{L}=-\frac{1}{4} G_{\rho \sigma}^{a} G^{\rho \sigma a}-\frac{1}{2} D_{\rho} \Phi^{a} D^{\rho} \Phi^{a}-\frac{1}{2} D_{\rho} \Psi^{a} D^{\rho} \Psi^{a},
$$

where $\rho, \sigma=0,1,2,5$. It follows that the multi-string ansatz is a static solution with $A_{0}^{a}=0$ and all time derivatives vanish. The solution in $3+1$ dimensions has the form

$$
\begin{aligned}
\Phi^{a} & =\mp \frac{1}{g} \delta^{a j} \partial_{j} \ln f_{S}, \\
\Psi^{k} & =\frac{1}{g} \epsilon^{k j} \partial_{j} \ln f_{S}, \\
A_{k}^{a} & =-\delta^{a 3} \frac{1}{g} \epsilon^{k j} \partial_{j} \ln f_{S},
\end{aligned}
$$

where $j, k=1,2$. This solution represents a multi-string configuration with sources at $\vec{a}_{i}, i=1,2 \ldots N$. By setting $e^{2 \phi}=e^{2 \phi_{0}} f_{S}$, we obtain from the fivebrane ansatz a neutral multi-string solution and an exact heterotic multi-string solution. The neutral single-string solution coincides with that of [119] in the far-field limit. The solitonic string tension $\widetilde{T}_{2}$ is given by $\widetilde{T_{6}}(2 \pi)^{4} \alpha^{2}$ and from $(2.137)$ is related to the fundamental string tension $T_{2}$ by

$$
\left(2 \pi R_{2}\right)^{2} \kappa_{4}^{2} T_{2} \widetilde{T_{2}}=n \pi
$$

This implies $\lambda_{i}=n_{i} \alpha^{\prime} / 2 \pi R_{2}^{2}$. Like the monopole, the lagrangian per unit length for the string solution is finite as a result of the cancellation of divergences between the gauge and gravitational sectors.

As in the multi-monopole case, it is straightforward to reduce the multi-string solution to a solution in the four-dimensional space (0125). The gauge field reduction is done in (6.37). In the gravitational sector, the reduction from ten to six dimensions is trivial, as the metric is flat in the subspace (6789). In going from six to four dimensions, we compactify the $x_{3}$ and $x_{4}$ directions and again follow the Kaluza-Klein procedure by replacing $g_{33}$ and $g_{44}$ with a scalar field $e^{-2 \sigma_{2}}$. The tree-level effective action reduces in four dimensions to

$$
S_{4}=\frac{1}{2 \kappa_{4}^{2}} \int d^{4} x \sqrt{-g} e^{-2 \phi-2 \sigma_{2}}\left(R+4(\partial \phi)^{2}+8 \partial \sigma_{2} \cdot \partial \phi+2\left(\partial \sigma_{2}\right)^{2}-e^{4 \sigma_{2}} \frac{N_{\rho} N^{\rho}}{2}\right),
$$


where $\rho=0,1,2,5$, where $N_{\rho}=H_{\rho 34}=\partial_{\rho} B$, and where $B=B_{34}$. The four-dimensional string soliton solution for this reduced action is then given by

$$
\begin{aligned}
e^{2 \phi}=e^{-2 \sigma_{2}} & =e^{2 \phi_{0}}\left(1-\sum_{i=1}^{N} \lambda_{i} \ln \left|\vec{x}-\vec{a}_{i}\right|\right) \\
d s^{2} & =-d t^{2}+d x_{5}^{2}+e^{2 \phi}\left(d x_{1}^{2}+d x_{2}^{2}\right), \\
N_{i} & = \pm \epsilon_{i j} \partial_{j} e^{2 \phi}
\end{aligned}
$$

We complete the family of solitons that can be obtained from the solutions of the 't Hooft ansatz by demanding that $f$ depend on only one coordinate, say $x^{1}$. We may now consistently assume that $x^{2}, x^{3}, x^{4}, x^{7}, x^{8}, x^{9}$ are compactified on a six-dimensional torus, where we shall take the $x^{2}, x^{3}$ and $x^{4}$ circles to have circumference $2 \pi R_{3}$ and the rest to have circumference $2 \pi \sqrt{\alpha^{\prime}}$, so that $(2 \pi)^{6} R_{3}^{3} \alpha^{\prime 3 / 2} \kappa_{4}^{2}=\kappa_{10}^{2}$. Then the solution of $f^{-1} \square f=0$ has domain wall structure with the "confining potential"

$$
f_{D}=1+\Lambda x_{1}
$$

where $\Lambda$ is a constant. By setting $e^{2 \phi}=e^{2 \phi_{0}} f_{D}$, we obtain from the fivebrane ansatz a neutral domain wall solution and an exact heterotic domain wall solution. The solitonic domain wall tension $\widetilde{T_{3}}$ is given by $\widetilde{T_{6}}\left(2 \pi{\sqrt{\alpha^{\prime}}}^{3}\right.$ and from $(2.137)$ is related to the fundamental string tension $T_{2}$ by

$$
\left(2 \pi R_{3}\right)^{3} \kappa_{4}^{2} T_{2} \widetilde{T_{3}}=n \pi
$$

This implies $\Lambda=n \alpha^{\prime} / 2 \pi R_{3}^{3}$. Like the monopole and string we cannot identify an explicit coset conformal field theory near each source. Again the reduction to $D=4$ is straightforward. In the gauge sector, the action reduces to $\mathrm{YM}+$ three scalar fields $\Phi, \Psi$ and $\Pi$. For the spacetime (0156) the solution for the fields is given by

$$
\begin{aligned}
\Phi^{1} & =\mp \frac{1}{g} \partial_{1} \ln f_{D}, \\
\Psi^{3} & =\frac{1}{g} \partial_{1} \ln f_{D}, \\
\Pi^{2} & =-\frac{1}{g} \partial_{1} \ln f_{D} \\
A_{\mu} & =0
\end{aligned}
$$


where $\mu=0,1,5,6$. In the gravitational sector the tree-level effective action in $D=4$ has the form

$$
S_{4}=\frac{1}{2 \kappa^{2}} \int d^{4} x \sqrt{-g} e^{-2 \phi-3 \sigma_{3}}\left(R+4(\partial \phi)^{2}+12 \partial \sigma_{3} \cdot \partial \phi+6\left(\partial \sigma_{3}\right)^{2}-e^{6 \sigma_{3}} \frac{P^{2}}{2}\right),
$$

where $P$ is a cosmological constant. Note that (6.44) is not obtained by a simple reduction of the ten-dimensional action owing to the nonvanishing of $H_{234}$. The four-dimensional domain wall solution for this reduced action is then given by

$$
\begin{aligned}
e^{2 \phi}=e^{-2 \sigma_{3}} & =e^{2 \phi_{0}}\left(1+\Lambda x_{1}\right), \\
d s^{2} & =-d t^{2}+d x_{5}^{2}+d x_{6}^{2}+e^{2 \phi} d x_{1}^{2}, \\
P & =\Lambda .
\end{aligned}
$$

A trivial change of coordinates reveals that the spacetime is, in fact, flat. Dilatonic domain walls with a flat spacetime have been discussed in a somewhat different context in $[120,121,122]$.

As for the fivebrane in $D=10$, the mass of the monopole, the mass per unit length of the string and the mass per unit area of the domain wall saturate a Bogomol'nyi bound with the topological charge. (In the case of the string and domain, wall, however, we must follow [19] and extrapolate the meaning of the ADM mass to non-asymptotically flat spacetimes.)

\subsection{String/string duality and $S \leftrightarrow T$}

Let us focus on the solitonic string configuration (6.40) in the case of a single source. In terms of the complex field

$$
\begin{aligned}
T & =T_{1}+i T_{2} \\
& =B_{34}+i e^{-2 \sigma} \\
& =B_{34}+i \sqrt{\operatorname{det} g_{m n}^{S}} \quad m, n=3,4,
\end{aligned}
$$

where $g_{M N}^{S}$ is the string $\sigma$-model metric, the solution for $n=1$ and $R_{2}=\sqrt{\alpha^{\prime}}$ takes the form (with $z=x_{1}+x_{2}$ )

$$
\begin{aligned}
T & =\frac{1}{2 \pi i} \ln \frac{z}{r_{0}} \\
d s^{2} & =-d t^{2}+d x_{5}^{2}-\frac{1}{2 \pi} \ln \frac{r}{r_{0}} d z d \bar{z}
\end{aligned}
$$


whereas both the four-dimensional (shifted) dilaton $\eta=\phi+\sigma$ and the four-dimensional two-form $B_{\mu \nu}$ are zero. In terms of the canonical metric $g_{\mu \nu}, T_{1}$ and $T_{2}$, the relevant part of the action takes the form

$$
S_{4}=\frac{1}{2 \kappa_{4}^{2}} \int d^{4} x \sqrt{-g}\left(R-\frac{1}{2 T_{2}^{2}} g^{\mu \nu} \partial_{\mu} T \partial_{\nu} \bar{T}\right)
$$

and is invariant under the $S L(2, R)$ transformation

$$
T \rightarrow \frac{a T+b}{c T+d}, \quad a d-b c=1
$$

The discrete subgroup $S L(2, Z)$, for which $a, b, c$ and $d$ are integers, is just a subgroup of the $O(6,22 ; Z)$ target space duality, which can be shown to be an exact symmetry of the compactified string theory at each order of the string loop perturbation expansion.

This $S L(2, Z)$ is to be contrasted with the $S L(2, Z)$ symmetry of the elementary fourdimensional solution of [19] and of section 3.3. In the latter solution $T_{1}$ and $T_{2}$ are zero, but $\eta$ and $B_{\mu \nu}$ are non-zero. The relevant part of the action is

$$
S_{4}=\frac{1}{2 \kappa_{4}^{2}} \int d^{4} x \sqrt{-g}\left(R-2 g^{\mu \nu} \partial_{\mu} \eta \partial_{\nu} \eta-\frac{1}{12} e^{-4 \eta} H_{\mu \nu \rho} H^{\mu \nu \rho}\right) .
$$

The equations of motion of this theory also display an $S L(2, R)$ symmetry, but this becomes manifest only after dualizing and introducing the axion field $a$ via

$$
\sqrt{-g} g^{\mu \nu} \partial_{\nu} a=\frac{1}{3 !} \epsilon^{\mu \nu \rho \sigma} H_{\nu \rho \sigma} e^{-4 \eta}
$$

Then in terms of the complex field

$$
\begin{aligned}
S & =S_{1}+i S_{2} \\
& =a+i e^{-2 \eta}
\end{aligned}
$$

the Dabholkar et al. fundamental string solution may be written

$$
\begin{aligned}
S & =\frac{1}{2 \pi i} \ln \frac{z}{r_{0}} \\
d s^{2} & =-d t^{2}+d x_{5}^{2}-\frac{1}{2 \pi} \ln \frac{r}{r_{0}} d z d \bar{z}
\end{aligned}
$$

Thus (6.47) and (6.53) are the same with the replacement $T \leftrightarrow S$. It has been conjectured that this second $S L(2, Z)$ symmetry may also be a symmetry of string theory $[111,108,109]$, but this is far from obvious order by order in the string loop expansion since it involves 
a strong/weak coupling duality $\eta \rightarrow-\eta$. What interpretation are we to give to these two $S L(2, Z)$ symmetries: one an obvious symmetry of the fundamental string and the other an obscure symmetry of the fundamental string?

Related issues are brought up in the recent interesting papers by Sen [100], Schwarz and Sen [101] and Binétruy [102]. In particular, Sen draws attention to the Dabholkar et al. string solution (6.53) and its associated $S L(2, Z)$ symmetry as supporting evidence in favor of the conjecture that $S L(2, Z)$ invariance may indeed be an exact symmetry of string theory. He also notes that the spectrum of electric and magnetic charges is consistent with the proposed $S L(2, Z)$ symmetry [100]..$^{\dagger}$

All of these observations fall into place if one accepts the proposal of Schwarz and Sen [101]: under string/fivebrane duality the roles of the target-space duality and the strong/weak coupling duality are interchanged! This proposal is entirely consistent with an earlier one that under string/fivebrane duality the roles of the $\sigma$-model loop expansion and the string loop expansion are interchanged [31]. In this light, the two $S L(2, Z)$ symmetries discussed above are just what one expects. From the string point of view, the $T$-field $S L(2, Z)$ is an obvious target space symmetry, manifest order by order in string loops whereas the $S$-field $S L(2, Z)$ is an obscure strong/weak coupling symmetry. From the fivebrane point of view, it is the $T$-field $S L(2, Z)$ which is obscure while the $S$-field $S L(2, Z)$ is an "obvious" target space symmetry. (This has not yet been proved except at the level of the low-energy field theory, however. It would be interesting to have a proof starting from the worldvolume of the fivebrane.) This duality of dualities is illustrated in Table 4.

$\dagger$ Sen also discusses the concept of a "dual string", but for him this is obtained from the fundamental string by an $S L(2, Z)$ transform. For us, a dual string is obtained by the replacement $S \leftrightarrow T$. 


\section{Fundamental string Dual string}

$\begin{array}{ccc}\text { Moduli } & T=b+i e^{-\sigma} & S=a+i e^{-\eta} \\ \text { Worldsheet coupling } & <e^{\sigma}>=\alpha^{\prime} / R^{2} & <e^{\eta}>=g^{2} \\ \text { Large/small radius } & R \rightarrow \alpha^{\prime} / R & g \rightarrow 1 / g \\ \text { T-duality } & O(6,22 ; Z) & S L(2, Z) \\ \text { Axion/dilaton } & S=a+i e^{-\eta} & T=b+i e^{-\sigma} \\ \text { Spacetime coupling } & <e^{\eta}>=g^{2} & <e^{\sigma}>=\alpha^{\prime} / R^{2} \\ \text { Strong/weak coupling } & g \rightarrow 1 / g & R \rightarrow \alpha^{\prime} / R \\ \text { S-duality } & S L(2, Z) & O(6,22 ; Z)\end{array}$

Table 4: Duality of dualities.

String theory requires two kinds of loop expansion: classical $\left(\alpha^{\prime}\right)$ worldsheet loops with expansion parameter $\left\langle e^{\sigma}>\right.$ where $\sigma$ is a modulus field, and quantum $(\hbar)$ spacetime loops with expansion parameter $\left\langle e^{\eta}>\right.$ where $\eta$ is the dilaton field. Introducing the axion field $a$ and another pseudoscalar modulus field $b$, four-dimensional string/string duality interchanges the roles of $S=a+i e^{-\eta}$ and $T=b+i e^{-\sigma}$, and hence interchanges classical and quantum. Thus this duality of dualities exhibited by four-dimensional strings is entirely consistent with the earlier result that ten-dimensional string/fivebrane duality interchanges the spacetime and worldsheet loop expansions [31], and is entirely consistent with the Dirac quantization rule (6.38) that follows from the string/fivebrane rule (1.2):

$$
8 G R^{2}=n \alpha^{\prime} \tilde{\alpha}^{\prime} \quad n=\text { integer },
$$

where $2 \pi \tilde{\alpha}^{\prime}$ is the inverse tension of the dual string. Thus, for $n=1$, we have

$$
\begin{aligned}
& <e^{\eta}>=g^{2}=8 G / \alpha^{\prime}=\tilde{\alpha}^{\prime} / R^{2} \\
& <e^{\sigma}>=\tilde{g}^{2}=8 G / \tilde{\alpha}^{\prime}=\alpha^{\prime} / R^{2}
\end{aligned}
$$

where $\tilde{g}$ is the dual string spacetime loop expansion parameter. Invariance of the Dirac quantization rule requires that an $S(T)$ transformation of the fundamental string be accompanied by a $T(S)$ transformation of the dual string.

Group theoretically, these dualities are given by $O(6,22 ; Z)$ in the case of $T$-duality and $S L(2, Z)$ in the case of $S$-duality. It has been suggested $[117,116,123,124]$ that these two kinds of duality should be united into a bigger group $O(8,24 ; Z)$ which contains both as 
subgroups (see section 6.8). This would have the bizarre effect of eliminating the distinction between classical and quantum.

This interchange in the roles of the $S$ and $T$ field in going from the string to the fivebrane has also been noted by Binétruy [102]. It is made more explicit when $S$ is expressed in terms of the variables appearing naturally in the fivebrane version

$$
\begin{aligned}
S & =S_{1}+i S_{2} \\
& =A_{034789}+i e^{-2 \eta}, \\
& =A_{034789}+i \sqrt{\operatorname{det} g_{m n}^{F}}, \quad m, n=3,4,6,7,8,9,
\end{aligned}
$$

where $g_{M N}^{F}=e^{-\phi / 3} g_{M N}^{S}$ is the fivebrane $\sigma$-model metric [26] and $A_{M N P Q R S}$ is the 6-form which couples to the 6 -dimensional worldvolume of the fivebrane, in complete analogy with (6.46).

Note, however, that unlike the Dabholkar et al. solution and the Greene et al. solution, the symmetric solution (6.37) also involves the non-abelian gauge fields $A_{\rho}, \Phi, \Psi$ whose interactions appear to destroy the $S L(2, Z)$. This remains a puzzle. (A generalization of the $D=4$ Dabholkar et al. solution involving gauge fields may also be possible by obtaining it as a soliton of the fivebrane theory. This would involve a $D=4$ analogue of the $D=10$ solution discussed in section 5.4.)

It may at first sight seem strange that a string can be dual to another string in $D=4$. After all, the usual formula relating the dimension of an extended object, $d$, to that of the dual object, $\tilde{d}$, is $\tilde{d}=D-d-2$. So one might expect string/string duality only in $D=6$ [31]. However, when we compactify $n$ dimensions and allow the dual object to wrap around $m \leq \tilde{d}-1$ of the compactified directions we find $\tilde{d}_{\text {effective }}=\tilde{d}-m=D_{\text {effective }}-d-2+(n-m)$, where $D_{\text {effective }}=D-n$. In particular for $D_{\text {effective }}=4, d=2, n=6$ and $m=4$, we find $\tilde{d}_{\text {effective }}=2$.

Thus the whole string/fivebrane duality conjecture is put in a different light when viewed from four dimensions. After all, our understanding of the quantum theory of fivebranes in $D=10$ is rather poor, whereas the quantum theory of strings in $D=4$ is comparatively well-understood (although we still have to worry about the monopoles and domain walls). In particular, the dual string will presumably exhibit the normal kind of mass spectrum with linearly rising Regge trajectories, since the classical ( $\hbar$-independent) string expression $\widetilde{T}_{6} L^{4} \times(\text { angular momentum) has dimensions of (mass })^{2}$, whereas the analogous classical expression for an uncompactified fivebrane is $\left(\widetilde{T}_{6}\right)^{1 / 5} \times($ angular momentum $)$ which 
has dimensions (mass) $)^{6 / 5}[17]$. Indeed, together with the observation that the $S L(2, Z)$ strong/weak coupling duality appears only after compactifying at least 6 dimensions, it is tempting to revive the earlier conjecture $[17,93]$ that the internal consistency of the fivebrane may actually require compactification.

\subsection{Kaluza-Klein black holes in string theory}

In this section, we extend the three solutions of section 6.5 to two-parameter solutions of the low-energy equations of the four-dimensional heterotic string, characterized by a mass per unit $p$-volume, $\mathcal{M}_{p+1}$, and magnetic charge, $g_{p+1}$, where $p=0,1$ or 2 . We show them to be special cases of the generic black $p$-branes discussed in section 3 . The neutral solitons discussed in section 6.5 are recovered in the extremal limit, $\sqrt{2} \kappa \mathcal{M}_{p+1}=g_{p+1}$ and are non-singular in the sense that the curvature singularity disappears when expressed in terms of the dual $\sigma$-model metric. The two-parameter solution extending the supersymmetric monopole corresponds to a magnetically charged black hole, while the solution extending the supersymmetric domain wall corresponds to a black membrane. By contrast, the two-parameter string solution does not possess a finite horizon and corresponds to a naked singularity.

All three solutions involve both the dilaton and the modulus fields, and are thus to be contrasted with pure dilaton solutions. In particular, the effective scalar coupling to the Maxwell field, $e^{-a \phi} F_{\mu \nu} F^{\mu \nu}$, gives rise to a new string black hole with $a=\sqrt{3}$, in contrast to the pure dilaton solution of the heterotic string which has $a=1$ $[52,56,125,126,127,128,129,130,131,132]$. It thus resembles the black hole previously studied in the context of Kaluza-Klein theories [53,54,55,133,134,135,52,130] which also has $a=\sqrt{3}$, and which reduces to the Pollard-Gross-Perry-Sorkin [55,133,134] magnetic monopole in the extremal limit. As we shall see, this is no coincidence, but a consequence of the target space duality that interchanges winding modes and Kaluza-Klein modes. In this connection, we recall [136], according to which $a>1$ black holes behave like elementary particles!

The fact that the heterotic string admits $a=\sqrt{3}$ black holes also has implications for string/fivebrane duality [17-26]. We shall show that electric/magnetic duality in $D=4$ may be seen as a consequence of string/fivebrane duality in $D=10$. 
We begin with the two-parameter black hole. The solution of the action (6.31) is given by

$$
\begin{aligned}
e^{-2 \Phi} & =e^{2 \sigma_{1}}=\left(1-\frac{r_{-}}{r}\right), \\
d s^{2} & =-\left(1-\frac{r_{+}}{r}\right)\left(1-\frac{r_{-}}{r}\right)^{-1} d t^{2}+\left(1-\frac{r_{+}}{r}\right)^{-1} d r^{2}+r^{2}\left(1-\frac{r_{-}}{r}\right) d \Omega_{2}^{2}, \\
F_{\theta \varphi} & =\sqrt{r_{+} r_{-}} \sin \theta
\end{aligned}
$$

where here, and throughout this section, we set the dilaton vev $\Phi_{0}$ equal to zero. This represents a magnetically charged black hole with event horizon at $r=r_{+}$and inner horizon at $r=r_{-}$. The magnetic charge and mass of the black hole are given by

$$
\begin{aligned}
g_{1} & =\frac{4 \pi}{\sqrt{2} \kappa}\left(r_{+} r_{-}\right)^{\frac{1}{2}}, \\
\mathcal{M}_{1} & =\frac{2 \pi}{\kappa^{2}}\left(2 r_{+}-r_{-}\right) .
\end{aligned}
$$

Changing coordinates via $y=r-r_{-}$and taking the extremal limit $r_{+}=r_{-}$yields:

$$
\begin{aligned}
& e^{2 \Phi}=e^{-2 \sigma_{1}}=\left(1+\frac{r_{-}}{y}\right) \\
& d s^{2}=-d t^{2}+e^{2 \Phi}\left(d y^{2}+y^{2} d \Omega_{2}^{2}\right) \\
& F_{\theta \varphi}=r_{-} \sin \theta
\end{aligned}
$$

which is just the neutral (i.e. no Yang-Mills) version of the supersymmetric monopole solution of section 6.5 which saturates the Bogomol'nyi bound $\sqrt{2} \kappa \mathcal{M}_{1} \geq g_{1}$.

Next we derive a two-parameter string solution which, however, does not possess a finite event horizon and consequently cannot be interpreted as a black string. A twoparameter family of solutions of the action (6.39) is now given by

$$
\begin{aligned}
e^{2 \Phi} & =e^{-2 \sigma_{2}}=(1+k / 2-\lambda \ln y), \\
d s^{2} & =-(1+k) d t^{2}+(1+k)^{-1}(1+k / 2-\lambda \ln y) d y^{2}+y^{2}(1+k / 2-\lambda \ln y) d \theta^{2}+d x_{3}^{2}, \\
F_{\theta} & =\lambda \sqrt{1+k} .
\end{aligned}
$$

Note that for $k \neq 0$ this describes a string with non-zero deficit angle whereas for $k=0$ we recover the supersymmetric string soliton solution of section 6.5 which, as shown in section 6.6, is dual to the elementary string solution of Dabholkar et al. The solution shown in (6.60) in fact represents a naked singularity, since the event horizon is pushed 
out to $r_{+}=\infty$, which agrees with the Horowitz-Strominger "no-4D-black-string" theorem $[50]$.

Finally, we consider the two-parameter black membrane solution. The two-parameter black membrane solution of the action (6.44) is then

$$
\begin{aligned}
e^{-2 \Phi} & =e^{2 \sigma_{3}}=\left(1-\frac{r}{r_{-}}\right) \\
d s^{2} & =-\left(1-\frac{r}{r_{+}}\right)\left(1-\frac{r}{r_{-}}\right)^{-1} d t^{2}+\left(1-\frac{r}{r_{+}}\right)^{-1}\left(1-\frac{r}{r_{-}}\right)^{-4} d r^{2}+d x_{2}^{2}+d x_{3}^{2}, \\
F & =-\left(r_{+} r_{-}\right)^{-1 / 2} .
\end{aligned}
$$

This solution represents a black membrane with event horizon at $r=r_{+}$and inner horizon at $r=r_{-}$. Changing coordinates via $y^{-1}=r^{-1}-r_{-}^{-1}$ and taking the extremal limit yields

$$
\begin{aligned}
e^{2 \Phi} & =e^{-2 \sigma_{3}}=\left(1+\frac{y}{r_{-}}\right), \\
d s^{2} & =-d t^{2}+d x_{2}^{2}+d x_{3}^{2}+e^{2 \Phi} d y^{2}, \\
F & =-\frac{1}{r_{-}},
\end{aligned}
$$

which is just the supersymmetric domain wall solution of section 6.5.

The black hole, string and domain wall solutions of the heterotic string shown above are nothing but the $(\tilde{d}=1, d=1, a=\sqrt{3}, \omega=-4 / 3),(\tilde{d}=2, d=0, a=2, \omega=-3 / 2)$ and $(\tilde{d}=3, d=-1, a=\sqrt{7}, \omega=-10 / 7)$ special cases of the solutions of section 3.8. To confirm this, it is sufficient to show that the three actions $S_{1}, S_{2}$ and $S_{3}$ may be cast into the form (3.3). This is achieved by transforming the metric $g_{\mu \nu}$ and scalars $\Phi$ and $\sigma_{i},(i=1,2,3)$, to the canonical metric $g_{\mu \nu}$ (can) and scalars $\phi$ and $\lambda$ via the following field redefinitions:

monopole

$$
\begin{aligned}
g_{\mu \nu} & =e^{\frac{1}{\sqrt{3}}(\sqrt{2} \lambda-\phi)} g_{\mu \nu}(\mathrm{can}), \\
\Phi & =\frac{1}{2 \sqrt{3}}\left(\frac{\lambda}{\sqrt{2}}-2 \phi\right), \\
\sigma_{1} & =\frac{1}{\sqrt{3}}\left(\frac{\lambda}{\sqrt{2}}+\phi\right) .
\end{aligned}
$$

string

$$
\begin{aligned}
g_{\mu \nu} & =e^{\lambda} g_{\mu \nu}(\text { can }), \\
\Phi & =\frac{1}{2}(\lambda-\phi), \\
\sigma_{2} & =\frac{1}{2} \phi .
\end{aligned}
$$


membrane

$$
\begin{aligned}
g_{\mu \nu} & =e^{\frac{1}{\sqrt{7}}(\sqrt{6} \lambda+\phi)} g_{\mu \nu}(\text { can }), \\
\Phi & =\frac{1}{2 \sqrt{7}}\left(\frac{3 \sqrt{3}}{\sqrt{2}} \lambda-2 \phi\right), \\
\sigma_{3} & =\frac{1}{\sqrt{7}}\left(-\frac{\lambda}{\sqrt{6}}+\phi\right) .
\end{aligned}
$$

Having done this, we can then set $\lambda=0$ to obtain the desired result. Note that by analytically continuing the general solution to the cases $d=0$ and $d=-1$, we are extrapolating the meaning of the ADM mass and topological charge to non-asymptotically flat spacetimes.

We note that the black hole solution corresponds to a Maxwell-scalar coupling $e^{-a \phi} F_{\mu \nu} F^{\mu \nu}$ with $a=\sqrt{3}$. This is to be contrasted with the pure dilaton black hole solutions of the heterotic string that have attracted much attention recently $[52,56,125,126,127,128,129,130,131,132]$ and have $a=1^{*}$. The case $a=\sqrt{3}$ also occurs when the Maxwell field and the scalar field $\phi$ arise from a Kaluza-Klein reduction of pure gravity from $D=5$ to $D=4$ :

$$
\hat{g}_{M N}=e^{\frac{\phi}{\sqrt{3}}}\left(\begin{array}{cc}
g_{\mu \nu}+e^{-\sqrt{3} \phi} A_{\mu} A_{\nu} & e^{-\sqrt{3} \phi} A_{\mu} \\
e^{-\sqrt{3} \phi} A_{\nu} & e^{-\sqrt{3} \phi}
\end{array}\right)
$$

where $\hat{g}_{M N}(M, N=0,1,2,3,4)$ and $g_{\mu \nu}(\mu, \nu=0,1,2,3)$ are the canonical metrics in 5 and 4 dimensions respectively. The resulting action is given by

$$
S=\frac{1}{2 \kappa^{2}} \int d^{4} x \sqrt{-g}\left[R-\frac{1}{2}(\partial \phi)^{2}-\frac{1}{4} e^{-\sqrt{3} \phi} F_{\mu \nu} F^{\mu \nu}\right]
$$

and it admits as an "elementary" solution the $a=\sqrt{3}$ black hole metric (6.57), but with the scalar field

$$
e^{-2 \phi}=\Delta_{-}^{\sqrt{3}}
$$

and the electric field

$$
\frac{1}{\sqrt{2} \kappa} e^{-\sqrt{3} \phi *} F_{\theta \varphi}=\frac{e}{4 \pi} \sin \theta
$$

* Contrary to some claims in the literature, the pure Reissner-Nordström black hole with $a=0$ is also a solution of the low energy heterotic string equations. This may be seen by noting that it provides a solution to $(N=2, D=4)$ supergravity which is a consistent truncation of toroidally compactified $N=1, D=10$ supergravity [137]. 
corresponding to an electric monopole with Noether charge $e$. This system also admits the topological magnetic solution with

$$
e^{-2 \phi}=\Delta_{-}^{-\sqrt{3}}
$$

and the magnetic field

$$
\frac{1}{\sqrt{2} \kappa} F_{\theta \varphi}=\frac{g}{4 \pi} \sin \theta
$$

corresponding to a magnetic monopole with topological magnetic charge $g$ obeying the Dirac quantization rule

$$
e g=2 \pi n, n=\text { integer. }
$$

In effect, it was for this reason that the $a=\sqrt{3}$ black hole was identified as a solution of the Type II string in [49], the fields $A_{\mu}$ and $\phi$ being just the abelian gauge field and the dilaton of $(N=2, D=10)$ supergravity which arises from Kaluza-Klein compactification of $(N=1, D=11)$ supergravity.

Some time ago, it was pointed out in [135] that $N=8$ supergravity, compactified from $D=5$ to $D=4$, admits an infinite tower of elementary states and that these elementary states fall into $N=8$ supermultiplets. They also pointed out that this theory admits an infinite tower of solitonic states which also fall into the same $N=8$ supermultiplets. The authors of [135] conjectured, á la Olive-Montonen [42], that there should exist a dual formulation of the theory for which the roles of electric elementary states and magnetic solitonic states are interchanged. It was argued in [49] that this electric/magnetic duality conjecture in $D=4$ could be reinterpreted as a particle/sixbrane duality conjecture in $D=10$.

To see this, consider the action dual to $S$, with $a=-\sqrt{3}$, for which the roles of Maxwell field equations and Bianchi identities are interchanged:

$$
\tilde{S}=\frac{1}{2 \kappa^{2}} \int d^{4} x \sqrt{-g}\left[R-\frac{1}{2}(\partial \phi)^{2}-\frac{1}{4} e^{\sqrt{3} \phi} \tilde{F}_{\mu \nu} \tilde{F}^{\mu \nu}\right],
$$

where $\tilde{F}_{\mu \nu}=e^{-\sqrt{3} \phi *} F_{\mu \nu}$. This is precisely the action obtained by double dimensional reduction of a dual formulation of $(D=10, N=2)$ supergravity in which the twoform $F_{M N}(M, N=0, \ldots, 9)$ is swapped for an 8-form $\tilde{F}_{M_{1} . . M_{8}}$, where $\tilde{F}_{\mu \nu}=\tilde{F}_{\mu \nu 456789}$. This dual action also admits both electric and magnetic monopole solutions but because the roles of field equations and Bianchi identities are interchanged, so are the roles of electric and magnetic. Since the 1-form and 7 -form potentials, which give rise to these 
2-form and 8-form field strengths, are those that couple naturally to the worldline of a point particle or the worldvolume of a 6-brane, we see that the Gibbons-Perry $(N=$ $8, D=4$ ) electric/magnetic duality conjecture may be re-expressed as a (Type II, $D=10$ ) particle/sixbrane duality conjecture. Indeed, the $D=10$ black sixbrane of [50] is simply obtained by adding 6 flat dimensions to the $D=4, a=\sqrt{3}$ magnetic black hole.

In general, the $N=8$ theory will admit black holes with mass $M$ and magnetic charge $g$ saturating the Bogomol'nyi bounds

$$
4 \pi G\left(M^{2}+\Sigma^{2}\right)=4 \pi G M^{2}\left(1+a^{2}\right)=g^{2}
$$

where $\Sigma=a M$ is the scalar charge.

The solutions presented in this section now allow us to discuss the $a=\sqrt{3}$ electric/magnetic duality from a totally different perspective from above. For concreteness, let us focus on generic toroidal compactification of the heterotic string. Instead of $N=8$ supergravity, the four-dimensional theory is now $N=4$ supergravity coupled to $22 N=4$ vector multiplets. The same dual Lagrangians (6.67) and (6.73) still emerge but with completely different origins. The Maxwell field $F_{\mu \nu}\left(\right.$ or $\left.\tilde{F}_{\mu \nu}\right)$ and the scalar field $\phi$ do not come from the $D=10$ 2-form (or 8-form) and dilaton of the Type II particle (or sixbrane), but rather from the $D=103$-form (or 7-form) and dilaton plus modulus field of the heterotic string (or heterotic fivebrane). Thus, the $D=4$ electric/magnetic duality can now be re-interpreted as a $D=10$ string/fivebrane duality! (Note that in addition to the 6 Maxwell fields coming from the $D=103$-form, there will be another 6 coming from the $D-10$ metric. Moreover, these are interchanged under an $O(6,6 ; Z)$ subgroup of the target-space duality discussed below. A truncation of a single $U(1)$ similar to (6.31) also yields $a \sqrt{3}$; a result well-known from Kaluza-Klein theory ${ }^{\dagger}$. That both kinds of black-hole solutions have $a=\sqrt{3}$ can thus be understood as a consequence of duality.)

$\dagger$ Gibbons discusses both the $a=1$ black hole of pure $N=4$ supergravity and the $a=\sqrt{3}$ Kaluza-Klein black hole in the same paper [52], as does Horowitz [130]. Moreover, black holes in pure $N=4$ supergravity are treated by Kallosh et al. [127-129]. The reader may therefore wonder why the $a=\sqrt{3} N=4$ black hole discussed here was overlooked. The reason is that pure $N=4$ supergravity does not admit the $a=\sqrt{3}$ solution; it is crucial that we include the $N=4$ vector multiplets in order to introduce the modulus fields. 
Because of the non-vanishing modulus field $g_{44}=e^{-2 \sigma}$, however, the $D=10$ black fivebrane solution is not obtained by adding 6 flat dimensions to the $D=4$ black hole. Rather the two are connected by wrapping the fivebrane around 5 of the 6 extra dimensions [99].

Of course, we have established only that these two-parameter configurations are solutions of the field theory limit of the heterotic string. Although the extreme one-parameter solutions are expected to be exact to all orders in $\alpha^{\prime}$, the same reasoning does not carry over to the new two-parameter solutions. It would be interesting to pursue conformal field theory arguments (see section 8), perhaps along the lines recently suggested in [132].

It would be also interesting to see whether the generalization of the one-parameter solutions of section 6.5 to the two-parameter solutions of this section can be carried out when we include the Yang-Mills coupling. This would necessarily involve giving up the selfduality condition on the Yang-Mills field strength, however, since the self-duality condition is tied to the extreme, $\sqrt{2} \kappa \mathcal{M}_{p+1}=g_{p+1}$, supersymmetric solutions.

Finally, there is the question of whether these solutions are peculiar to the toroidal compactification or whether they survive in more realistic orbifold or Calabi-Yau models [138]. Although the actions $S_{1}, S_{2}$ and $S_{3}$ were originally derived in the context of the torus [99], they also appear in a large class of $N=1$ supergravity theories [139].

\subsection{Massive string states as extreme black holes}

The idea that elementary particles might behave like black holes is not a new one [140]. Intuitively, one might expect that a pointlike object whose mass exceeds the Planck mass, and whose Compton wavelength is therefore less than its Schwarzschild radius, would exhibit an event horizon. In the absence of a consistent quantum theory of gravity, however, such notions would always remain rather vague. Superstring theory, on the other hand, not only predicts such massive states but may provide us with a consistent framework in which to discuss them. In this section we confirm the claims of the previous section that certain massive excitations of four-dimensional superstrings are indeed black holes. Of course, non-extreme black holes would be unstable due to the Hawking effect. To describe stable elementary particles, therefore, we must focus on extreme black holes whose masses saturate a Bogomol'nyi bound *. We therefore remain agnostic concerning the stronger

* The relationship between extremal black holes and the gravitational field around some of the elementary string states has also been discussed in $[141,108]$. 
claims $[142,143]$ that all black holes are single string states or, conversely, that all massive string states are black holes.

We have just seen that this theory exhibits both electrically and magnetically charged black hole solutions corresponding to scalar-Maxwell parameter $a=0,1, \sqrt{3}$. In other words, by choosing appropriate combinations of dilaton and moduli fields to be the scalar field $\phi$ and appropriate combinations of the field strengths and their duals to be the Maxwell field $F$, the field equations can be consistently truncated to a form given by the Lagrangian

$$
\mathcal{L}=\frac{1}{32 \pi} \sqrt{-g}\left[R-\frac{1}{2}(\partial \phi)^{2}-\frac{1}{4} e^{-a \phi} F^{2}\right]
$$

for these three values of $a$. (A consistent truncation is defined to be one for which all solutions of the truncated theory are solutions of the original theory). In the case of zero angular momentum, the bound between the black hole ADM mass $m$, and the electric charge $Q=\int e^{-a \phi} \tilde{F} / 8 \pi$, where a tilda denotes the dual, is given by

$$
m^{2} \geq Q^{2} / 4\left(1+a^{2}\right)
$$

where, for simplicity, we have set the asymptotic value of $\phi$ to zero. The $a=0$ case yields the Reissner-Nordstrom solution which, notwithstanding contrary claims in the literature, does solve the low-energy string equations. The $a=1$ case yields the dilaton black hole $[52,125,132]$. The $a=\sqrt{3}$ case corresponds to the Kaluza-Klein black hole and the "winding" black hole [144] which are related to each other by $T$-duality. The Kaluza-Klein solution has been known for some time [52] but only recently recognized [144] as a heterotic string solution.

Let us denote by $N_{L}$ and $N_{R}$ the number of left and right oscillators respectively. We shall consider the Schwarz-Sen $[115,107] O(6,22 ; Z)$ invariant spectrum of elementary electrically charged massive $N_{R}=1 / 2$ states of this four-dimensional heterotic string, and show that the spin zero states correspond to extreme limits of black hole solutions which preserve $1 / 2$ of the spacetime supersymmetries. By supersymmetry, the black hole interpretation then applies to all members of the $N=4$ supermultiplet $[145,146]$, which has $s_{\max }=1$. For a subset of states the low-energy string action can be truncated to (6.75). The scalar-Maxwell parameter is given by $a=\sqrt{3}$ for $N_{L}=1$ and $a=1$ for $N_{L}>1$ (and vanishing left-moving internal momenta). The other states with $N_{L}>1$ are extreme black holes too, but are not described by a single scalar truncation of the type (6.75). The $N=4$ supersymmetry algebra possesses two central charges $Z_{1}$ and $Z_{2}$. The 
$N_{R}=1 / 2$ states correspond to that subset of the full spectrum that belong to the 16 complex dimensional $\left(s_{\max } \geq 1\right)$ representation of the $N=4$ supersymmetry algebra, are annihilated by half of the supersymmetry generators and saturate the strong Bogomol'nyi bound $m=\left|Z_{1}\right|=\left|Z_{2}\right|$. As discussed in [37,43,115,107], the reasons for focussing on this $N=4$ theory, aside from its simplicity, is that one expects that the allowed spectrum of electric and magnetic charges is not renormalized by quantum corrections, and that the allowed mass spectrum of particles saturating the Bogomol'nyi bound is not renormalized either. As discussed in $[115,107]$ only a subset of the conjectured spectrum corresponds to elementary string states. First of all these states will be only electrically charged, i.e. $\beta=0$, but there will be restrictions on $\alpha$ too. Without loss of generality let us focus on a compactification with $M^{0}=I$ and ${\lambda_{2}}^{0}=1$. Any other toroidal compactifications can be brought into this form by $O(6,22)$ transformations and a constant shift of the dilaton. The mass formula (6.22) now becomes

$$
m^{2}=\frac{1}{16} \alpha^{a}(I+L)_{a b} \alpha^{b}=\frac{1}{8}\left(\alpha_{R}\right)^{2}
$$

with $\alpha_{R}=\frac{1}{2}(I+L) \alpha$ and $\alpha_{L}=\frac{1}{2}(I-L) \alpha$. In the string language $\alpha_{R(L)}$ are the right(left)moving internal momenta. The mass of a generic string state in the Neveu-Schwarz sector (which is degenerate with the Ramond sector) is given by

$$
m^{2}=\frac{1}{8}\left\{\left(\alpha_{R}\right)^{2}+2 N_{R}-1\right\}=\frac{1}{8}\left\{\left(\alpha_{L}\right)^{2}+2 N_{L}-2\right\} .
$$

A comparison of (6.77) and (6.78) shows that the string states satisfying the Bogomol'nyi bound all have $N_{R}=1 / 2$. One then finds

$$
N_{L}-1=\frac{1}{2}\left(\left(\alpha_{R}\right)^{2}-\left(\alpha_{L}\right)^{2}\right)=\frac{1}{2} \alpha^{T} L \alpha,
$$

leading to $\alpha^{T} L \alpha \geq-2$. We shall now show that extreme black holes with $a=\sqrt{3}$ are string states with $\alpha^{T} L \alpha$ null $\left(N_{L}=1\right)$ and those with $a=1$ are string states with $\alpha^{T} L \alpha$ spacelike $\left(N_{L}>1\right)$. We have been unable to identify solutions of the low-energy field equations of (6.6) corresponding to states with $\alpha^{T} L \alpha$ timelike $\left(N_{L}<1\right){ }^{\dagger}$

Let us first focus on the $a=\sqrt{3}$ black hole. To identify it as a state in the spectrum we have to find the corresponding charge vector $\alpha$ and to verify that the masses calculated by

$\dagger$ In the non-abelian theory Sen [107] identifies these states with the electric analogues of BPS monopoles. 
the formulas (6.76) and (6.22) are identical. The action (6.6) can be consistently truncated by keeping the metric $g_{\mu \nu}$, just one field strength $\left(F=F^{1}\right.$, say), and one scalar field $\phi$ via the ansatz $\Phi=\phi / \sqrt{3}$ and $M_{11}=e^{2 \phi / \sqrt{3}}=M_{77}^{-1}$. All other diagonal components of $M$ are set equal to unity and all non-diagonal components to zero. Now (6.6) reduces to $(6.75)$ with $a=\sqrt{3}$. (This yields the electric and magnetic Kaluza-Klein (or "F") monopoles. This is not quite the truncation chosen in [144], where just $F^{7}$ was retained and $M_{11}=e^{-2 \phi / \sqrt{3}}=M_{77}^{-1}$. This yields the electric and magnetic winding (or " $H$ ") monopoles. However, the two are related by $T$-duality). We shall restrict ourselves to the purely electrically charged solution with charge $Q=1$, since this one is expected to correspond to an elementary string excitation. The charge vector $\alpha$ for this solution is obviously given by $\alpha^{a}=\delta^{a, 1}$ with $\alpha^{T} L \alpha=0$. Applying (6.22) for the mass of the state we find $m^{2}=1 / 16=Q^{2} / 16$, which coincides with (6.76) in the extreme limit. This agreement confirms the claim that this extreme $a=\sqrt{3}$ black hole is a state in the Sen-Schwarz spectrum and preserves 2 supersymmetries.

Next we turn to the $a=1$ black hole. The theory is consistently truncated by keeping the metric, $F=F^{1}=F^{7}$ and setting $M=I$. The only non-vanishing scalar is the dilaton $\Phi \equiv \phi$. Now (6.6) reduces to (6.75) with $a=1$ but $Q^{2}=2$. An extreme $a=1$ black hole with electric charge $Q$ is then represented by the charge configuration $\alpha^{a}=\delta^{a, 1}+\delta^{a, 7}$. Applying (6.22) we find $m^{2}=1 / 4=Q^{2} / 8$ which coincides with (6.76) in the extreme limit. Therefore the $a=1$ extreme solution is also in the spectrum, and has $\alpha^{T} L \alpha=2$ or $N_{L}=2$.

Although physically very different, we can see with hindsight that both the $a=\sqrt{3}$ and $a=1$ black holes permit a uniform mathematical treatment by noting that both may be obtained from the Schwarzschild solution by performing an $[O(6,1) \times O(22,1)] /[O(6) \times$ $O(22)$ transformation [108]. The 28 parameters of this transformation correspond to the 28 $U(1)$ charges. If $\gamma$ and $\mathbf{u}$ correspond to the boost angle and a 22 dimensional unit vector respectively, associated with $O(22,1) / O(22)$ transformations, $\delta$ and $\mathbf{v}$ denote the boost angle and the 6 dimensional unit vector respectively, associated with the $O(6,1) / O(6)$ transformations, and $m_{0}$ is the mass of the original Schwarzschild black hole, then the mass and charges of the new black hole solution are given by [108]:

$$
\begin{aligned}
m & =\frac{1}{2} m_{0}(1+\cosh \gamma \cosh \delta), \\
\alpha_{L} & =\sqrt{2} m_{0} \cosh \delta \sinh \gamma \mathbf{u}, \\
\alpha_{R} & =\sqrt{2} m_{0} \cosh \gamma \sinh \delta \mathbf{v} .
\end{aligned}
$$


(Note that the convention about $R$ and $L$ of [108] is opposite to the one used in this section). Black holes with $\alpha^{T} L \alpha=0$ are generated by setting $\gamma=\delta$, whereas black holes with $\alpha^{T} L \alpha>0$ are generated by setting $\gamma<\delta$. The Bogomol'nyi bound given in (6.77) corresponds to $m^{2}=\left(\alpha_{R}\right)^{2} / 8$. This bound is saturated by taking the limit where the mass $m_{0}$ of the original Schwarzschild black hole approaches 0 and the parameter $\delta$ approaches $\infty$, keeping the product $m_{0} \sinh \delta$ fixed. As discussed in [108], this is precisely the extremal limit. Thus we see that extremal black holes satisfy the Bogomol'nyi relation, both for $\alpha^{T} L \alpha=0$ and $\alpha^{T} L \alpha>0$.

From the above $a=\sqrt{3}$ solution we can generate the whole set of supersymmetric black hole solutions with $\alpha^{T} L \alpha=0$ in the following way: first we note that we are interested in constructing black hole solutions with different charges but with fixed asymptotic values of $M$ (which here has been set to the identity). Thus we are not allowed to make $O(6,22)$ transformations that change the asymptotic value of $M$. This leaves us with only an $O(6) \times O(22)$ group of transformations. The effect of these transformations acting on the parameters given in (6.80) above is to transform the vectors $\mathbf{u}$ and $\mathbf{v}$ by $O(22)$ and $O(6)$ transformations respectively without changing the parameters $\gamma$ and $\delta$. Now, the original $a=\sqrt{3}$ solution corresponds to a choice of parameters $\gamma=\delta, \mathbf{u}^{m}=\delta_{m 1}$ and $\mathbf{v}^{m}=\delta_{k 1}$. It is clear that an $O(6) \times O(22)$ transformation can rotate $\mathbf{u}$ and $\mathbf{v}$ to arbitrary 22 and 6 dimensional unit vectors respectively, wothout changing $\gamma$ and $\delta$. Since this corresponds to the most general charge vector satisfying $\alpha^{T} L \alpha=0$, we see that the $O(6) \times O(22)$ transformation can indeed generate an arbitrary black hole solution with $\alpha^{T} L \alpha=0$ starting from the original $a=\sqrt{3}$ solution. This clearly leaves the mass invariant, but the new charge vector $\alpha^{\prime}$ will in general not be located on the lattice. To find a state in the allowed charge spectrum we have to rescale $\alpha^{\prime}$ by a constant $k$ so that $\alpha^{\prime \prime}=k \alpha^{\prime}$ is a lattice vector. Clearly the masses calculated by (6.76) and (6.22) still agree (this is obvious by reversing the steps of rotation and rescaling), leading to the conclusion that all states obtained in this way preserve $1 / 2$ of the supersymmetries. Therefore all states in the spectrum belonging to $s_{\max }=1$ supermultiplets for which $N_{R}=1 / 2, N_{L}=1$ are extreme $a=\sqrt{3}$ black holes.

Let us now turn to the case of the $a=1$ solution. In this case the original solution corresponds to the choice of parameters $\gamma=0, \mathbf{v}^{m}=\delta_{m 1}$. (For $\gamma=0$, the parameter $\mathbf{u}$ is irrelevant). An $O(6) \times O(22)$ transformation can rotate $\mathbf{v}$ to any other 6 dimensional unit vector, but it cannot change the parameters $\delta$ and $\gamma$. As a result, the final solution will continue to have $\gamma=0$ and hence $\alpha_{L}=0$. Since this does not represent the most general 
charge vector $\alpha$, with $\alpha^{T} L \alpha>0$, we see that the most general black hole representing states with $\alpha^{T} L \alpha>0$ is not obtained in this way even after rescaling. The missing states with $\alpha_{L} \neq 0$ are constructed by choosing $\gamma$ so that $\tanh ^{2} \gamma=\alpha_{L}^{2} / \alpha_{R}^{2}$, and $\mathbf{u}, \mathbf{v}$ as for the $a=\sqrt{3}$ case, followed by a suitable $O(6) \times O(22)$ rotation. Clearly, those solutions are extreme black holes too. However, for these solutions a truncation to an effective action of the form (6.75) is not possible. The following picture arises: for a fixed value of $\alpha_{R}^{2}, \alpha_{L}^{2}$ can vary in the range $\alpha_{R}^{2} \geq \alpha_{L}^{2} \geq 0$. The boundary states are described by the well-known $a=\sqrt{3}\left(\alpha_{R}^{2}=\alpha_{L}^{2}\right)$ and $a=1\left(\alpha_{L}^{2}=0\right)$ black holes, whereas the states in between cannot be related to a single scalar-Maxwell parameter $a$. But all solutions preserve $1 / 2$ of the supersymmetries.

It should also be clear that the purely magnetic extreme black hole solutions [144] obtained from the above by the replacements $\phi \rightarrow-\phi, \alpha \rightarrow \beta$ will also belong to the Schwarz-Sen spectrum of solitonic states. Starting from either the purely electric or purely magnetic solutions, dyonic states in the spectrum which involve non-vanishing axion field $\Psi$ can then be obtained by $S L(2, Z)$ transformations. Specifically, a black hole with charge vector $(\alpha, 0)$ will be mapped into ones with charges $(a \alpha, c \alpha)$ with the integers $a$ and $c$ relatively prime $[115,107]$.

Not all black hole solutions of (6.6) belong to the Sen-Schwarz spectrum, however. Let us first consider the Reissner-Nordstrom solution. Since this black hole solves the equations of $N=2$ supergravity, whose bosonic sector is pure Einstein-Maxwell, it solves (6.6) as well. The required consistent truncation is obtained by keeping $g_{\mu \nu}, F=F^{1}=F^{7}=\tilde{F}^{2}=\tilde{F}^{8}$ and setting $\Phi=0, M=1$. Now (6.6) effectively reduces to (6.75) with $a=0$ but $Q^{2}=4$. On the other hand, if it were in the Schwarz-Sen spectrum its charge vectors would be given by $\alpha^{a}=\delta^{a, 1}+\delta^{a, 7}$ with $\alpha^{T} L \alpha=2$ and $\beta^{a}=\delta^{a, 2}+\delta^{a, 8}$ with $\beta^{T} L \beta=2$. Applying (6.22) for the mass of the state we find $m^{2}=1 / 2$, which disagrees with the result $m^{2}=1$ obtained from the extreme limit of (6.76) Sंo the test fails and the $a=0$ black hole does not belong to the Schwarz-Sen spectrum. This was only to be expected since it breaks $3 / 4$ of the supersymmetries and hence saturates the weaker Bogomol'nyi bound $m=\left|Z_{1}\right|,\left|Z_{2}\right|=0$ [127]. Such black holes belong to the 32 complex dimensional $\left(s_{\max }=3 / 2\right)$ supermultiplet. We see no reason to exclude these states from the full string spectrum, however. Another example of a black hole solution not in the Schwarz-Sen spectrum is the $a=1$ dilaton black hole of $[125,132]$ where the only non-vanishing gauge field is $F^{13}$. This has mass $m^{2}=Q^{2} / 8$ but according to (6.22) its mass would vanish. Again, this contradiction is only to be expected since this solution breaks all the supersymmetries, in contrast with 
the $F=F^{1}=F^{7}$ embedding discussed above. We do not know whether such black holes saturating no Bogomol'nyi bound $\left(m>\left|Z_{1}\right|,\left|Z_{2}\right|\right)$, which include the neutral Schwarzschild black holes $\left(Z_{1}=Z_{2}=0\right)$, are also in the string spectrum. States with these quantum numbers would belong to the 256 dimensional $\left(s_{\max } \geq 2\right)$ supermultiplets. According to [145], however, black holes breaking all the supersymmetries do not themselves form supermultiplets. This would appear to contradict the claim that all black holes are string states.

In the supersymmetric case, all values of $a$ lead to extreme black holes with zero entropy but their temperature is zero, finite or infinite according as $a<1, a=1$ or $a>1$, and so in [136] the question was posed: can only $a>1$ scalar black holes describe elementary particles? We have not definitively answered this question but a tentative response would be as follows. First we note that the masses and charge vectors are such that the lightest $a=0$ black hole may be regarded as a bound state (with zero binding energy) of two lightest $a=1$ black holes which in turn can each be regarded as bound states (again with zero binding energy) of two lightest $a=\sqrt{3}$ black holes. Thus if by elementary particle one means an object which cannot be regarded as a bound state, then indeed extreme scalar black holes with $a>1$ are the only possibilty, but if one merely means a state in the string spectrum then $a \leq 1$ extreme scalar black holes are also permitted.

We have limited ourselves to $N_{R}=1 / 2$ supermultiplets with $s_{\min }=0$. Having established that the $s=0$ member of the multiplet is an extreme black hole, one may then use the fermionic zero modes to perform supersymmetry transformations to generate the whole supermultiplet of black holes $[145,146]$ with the same mass and charges. Of course, there are $N_{R}=1 / 2$ multiplets with $s_{\min }>0$ coming from oscillators with higher spin and our arguments have nothing to say about whether these are also extreme black holes. They could be naked singularities. Indeed, although in this paper we have focussed primarily on identifying certain massive heterotic string states with extreme black holes, perhaps equally remarkable is that these elementary string states can be described at all by solutions of the supergravity theory. In a field theory, as opposed to a string theory, one is used to having as elementary massive states only the Kaluza-Klein modes with $s_{\max }=2$. However, as we have already seen, the winding states (usually thought of as intrinsically stringy) are on the same footing as Kaluza-Klein states as far as solutions are concerned, so perhaps the same is true for the $s>2$ states.

None of the spinning $N_{R}=1 / 2$ states is described by extreme rotating black hole metrics because they obey the same Bogomol'nyi bound as the $s_{\min }=0$ states, whereas 
the mass formula for an extreme rotating black hole depends on the angular momentum $J$. Moreover, it is the fermion fields which carry the spin in the $s_{\min }=0$ supermultiplet. (For the $a=0$ black hole, they yield a gyromagnetic ratio $g=2[146]$; the $a=\sqrt{3}$ and $a=1$ superpartner $g$-factors are unknown to us). It may be that there are states in the string spectrum described by the extreme rotating black hole metrics but if so they will belong to the $N_{R} \neq 1 / 2$ sector**. Since, whether rotating or not, the black hole solutions are still independent of the azimuthal angle and independent of time, the supergravity theory is effectively two-dimensional and therefore possibly integrable. This suggests that the spectrum should be invariant under the larger duality $O(8,24 ; Z)$ [117], which combines $S$ and $T$. The corresponding Kac-Moody extension would then play the role of the spectrum generating symmetry [150].

** The gyromagnetic and gyroelectric ratios of the states in the heterotic string spectrum would then have to agree with those of charged rotating black hole solutions of the heterotic string. This is indeed the case: the $N_{L}=1$ states [147] and the rotating $a=\sqrt{3}$ black holes [148] both have $g=1$ whereas the $N_{L}>1$ states [143] and the rotating $a=1$ [131] (and $a=0$ [149]) black holes both have $g=2$. In fact, it was the observation that the Regge formula $J \sim m^{2}$ also describes the mass/angular momentum relation of an extreme rotating black hole which first led Salam [140] to imagine that elementary particles might behave like black holes! 


\section{Dynamics of string solitons}

\subsection{Metric on moduli space}

All the soliton solutions we have discussed so far have the property, like BPS magnetic monopoles, that they exert zero static force on each other and can be superposed to form multi-soliton solutions with arbitrarily variable collective coordinates. Since these static properties are also possessed by fundamental strings winding around an infinitely large compactified dimension, it was conjectured in [151] that the elementary string is actually the exterior solution for an infinitely long fundamental string. In this section we show that, in contradistinction to the BPS case, the velocity-dependent forces between these string solitons also vanish, at least to order $\beta^{2}$ (where $\beta$ is the velocity). We also argue that this phenomenon provides further, dynamical evidence for the identification of the elementary string solution with the underlying fundamental string by comparing the scattering of the elementary solutions with expectations from a Veneziano amplitude computation for macroscopic fundamental strings [22].

As shown in (3.52), the static ansatz leads to a vanishing velocity-dependent force for a test $(d-1)$-brane propagating in the background of an elementary $(d-1)$-brane. By duality, this result also holds for test $(\tilde{d}-1)$-branes propagating in the background of a solitonic $(\tilde{d}-1)$-brane. As this is a rather surprising result, we would like to compute the metric on moduli space for these solitons. The geodesics of this metric represent the motion of quasi-static solutions in the static solution manifold and in the absence of a full time dependent solution provide a good approximation to the low-energy dynamics of the solitons. In all cases the metric is found to be flat in agreement with the testsoliton approximation, which again implies vanishing dynamical force in the low-velocity limit. Here we summarize the computation for the metric on moduli space for monopoles discussed in $[152,97]$.

Manton's prescription [153] for the study of soliton scattering may be summarized as follows. We first invert the constraint equations of the system. The resultant time dependent field configuration does not in general satisfy the full time dependent field equations, but provides an initial data point for the fields and their time derivatives. 
Another way of saying this is that the initial motion is tangent to the set of exact static solutions. The kinetic action obtained by replacing the solution to the constraints into the action defines a metric on the parameter space of static solutions. This metric defines geodesic motion on the moduli space [153].

A calculation of the metric on moduli space for the scattering of BPS monopoles and a description of its geodesics was worked out by Atiyah and Hitchin [154]. Several interesting properties of monopole scattering were found, such as the conversion of monopoles into dyons and the right angle scattering of two monopoles on a direct collision course $[154,155]$. The configuration space is found to be a four-dimensional manifold $M_{2}$ with a self-dual Einstein metric.

Here we adapt Manton's prescription to study the dynamics of the heterotic string monopoles discussed in section 6.5. We follow essentially the same steps that Manton outlined for monopole scattering, but take into account the peculiar nature of the string effective action. Since we work in the low-velocity limit, our kinematic analysis is nonrelativistic.

We first solve the constraint equations for the monopoles. These equations are simply the $(0 j)$ components of the tree-level equations of motion

$$
\begin{aligned}
R_{0 j}-\frac{1}{4} H_{0 j}^{2}+2 \nabla_{0} \nabla_{j} \phi & =0, \\
-\frac{1}{2} \nabla_{k} H^{k}{ }_{0 j}+H_{0 j}{ }^{k} \partial_{k} \phi & =0 .
\end{aligned}
$$

We wish to find an $O(\beta)$ solution to the above equations which represents a quasi-static version of the neutral multi-monopole solution (i.e. a multi-monopole solution with time dependent $\vec{a}_{i}$ ). We consider the neutral case as it was argued in [156] that the the cancellation of gauge and gravitational corrections in the static action for the symmetric case also manifests itself in the dynamics, at least to $O\left(\alpha^{\prime}\right)$. Hence the scattering of symmetric string monopoles is expected to be similar to that of neutral string monopoles. Also, we use the solution (6.24) with $e^{2 \phi}=e^{2 \phi_{0}} f_{M}$, with $f_{M}$ given in (6.25) and compactify only the directions $x^{5}, x^{6}, x^{7}, x^{8}, x^{9}$. In other words, we do not make the replacement $g_{44}=e^{-2 \sigma}$ to obtain the completely compactified version (6.32), although the results in both cases are identical, in order to more easily keep track of the terms in the former case. We give each monopole an arbitrary transverse velocity $\vec{\beta}_{n}$ in the (123) subspace of the four-dimensional transverse space and see what corrections to the fields are required by the constraints. The 
vector $\vec{a}_{n}$ representing the position of the $n$th monopole in the three-space (123) is given by

$$
\vec{a}_{n}(t)=\vec{A}_{n}+\vec{\beta}_{n} t
$$

where $\vec{A}_{n}$ is the initial position of the $n$th monopole. Note that at $t=0$ we recover the exact static multi-monopole solution. Our solution to the constraints will adjust our quasi-static approximation so that the initial motion in the parameter space is tangent to the initial exact solution at $t=0$. The $O(\beta)$ solution to the constraints is given by

$$
\begin{aligned}
e^{2 \phi(\vec{x}, t)} & =1+\sum_{n=1}^{N} \frac{m_{n}}{\left|\vec{x}-\vec{a}_{n}(t)\right|}, \\
g_{00} & =-1, \quad g^{00}=-1, \quad g_{i j}=e^{2 \phi} \delta_{i j}, \quad g^{i j}=e^{-2 \phi} \delta_{i j}, \\
g_{0 i} & =-\sum_{n=1}^{N} \frac{m_{n} \vec{\beta}_{n} \cdot \hat{x}_{i}}{\left|\vec{x}-\vec{a}_{n}(t)\right|}, \quad g^{0 i}=e^{-2 \phi} g_{0 i}, \\
H_{i j k} & =\epsilon_{i j k m} \partial_{m} e^{2 \phi} \\
H_{0 i j} & =\epsilon_{i j k m} \partial_{m} g_{0 k}=\epsilon_{i j k m} \partial_{k} \sum_{n=1}^{N} \frac{m_{n} \vec{\beta}_{n} \cdot \hat{x}_{m}}{\left|\vec{x}-\vec{a}_{n}(t)\right|}
\end{aligned}
$$

where $i, j, k, m=1,2,3,4$, all other metric components are flat, all other components of $H$ vanish, the $\vec{a}_{n}(t)$ are given by (7.2) and we use a flat space $\epsilon$-tensor. Note that $g_{00}, g_{i j}$ and $H_{i j k}$ are unaffected to order $\beta$. Also note that we can interpret the monopoles as either strings in the space (01234) or point objects in the three-dimensional subspace (0123).

The kinetic Lagrangian is obtained by replacing the expressions for the fields in (7.3) into the string $\sigma$-model action (3.81) for $D=5^{*}$. Since (7.3) is a solution to order $\beta$, the leading order terms in the action (after the quasi-static part) are of order $\beta^{2}$. The $O(\beta)$ terms in the solution give $O\left(\beta^{2}\right)$ terms when replaced in the kinetic action. Collecting all $O\left(\beta^{2}\right)$ terms in (3.81) we get the following kinetic Lagrangian density for the volume term:

$$
\begin{aligned}
& \mathcal{L}_{k i n}=-\frac{1}{2 \kappa^{2}}\left(4 \dot{\phi} \vec{M} \cdot \vec{\nabla} \phi-e^{-2 \phi} \partial_{i} M_{j} \partial_{i} M_{j}-e^{-2 \phi} M_{k} \partial_{j} \phi\left(\partial_{j} M_{k}-\partial_{k} M_{j}\right)\right. \\
& \left.+4 M^{2} e^{-2 \phi}(\vec{\nabla} \phi)^{2}+2 \partial_{t}^{2} e^{2 \phi}-4 \partial_{t}(\vec{M} \cdot \vec{\nabla} \phi)-4 \vec{\nabla} \cdot(\dot{\phi} \vec{M})\right),
\end{aligned}
$$

* Strictly speaking one must add to (3.81) a surface term to cancel the double derivative terms in the action $[157,158,159,160,152]$ however the addition of this term introduces only flat kinetic terms and thus presents no nontrivial contribution to the metric on moduli space. 
where $\vec{M} \equiv-\sum_{n=1}^{N} \frac{m_{n} \vec{\beta}_{n}}{\left|\vec{x}-\vec{a}_{n}(t)\right|}$. Henceforth let $\vec{X}_{n} \equiv \vec{x}-\vec{a}_{n}(t)$. The last three terms in (7.4) are time-surface or space-surface terms which vanish when integrated. The kinetic Lagrangian $L_{k i n}=\int d^{3} x \mathcal{L}_{k i n}$ for monopole scattering converges everywhere. This can be seen simply by studying the limiting behaviour of $L_{k i n}$ near each monopole. For a single monopole at $r=0$ with magnetic charge $m$ and velocity $\beta$, we collect the logarithmically divergent pieces and find that they cancel:

$$
\frac{m \beta^{2}}{2} \int r^{2} d r d \theta \sin \theta d \phi\left(-\frac{1}{r^{3}}+\frac{3 \cos ^{2} \theta}{r^{3}}\right)=0 .
$$

We now specialize to the case of two identical monopoles of magnetic charge $m_{1}=$ $m_{2}=m$ and velocities $\vec{\beta}_{1}$ and $\vec{\beta}_{2}$. Let the monopoles be located at $\vec{a}_{1}$ and $\vec{a}_{2}$. Our moduli space consists of the configuration space of the relative separation vector $\vec{a} \equiv \vec{a}_{2}-\vec{a}_{1}$. The most general kinetic Lagrangian can be written as

$$
\begin{aligned}
L_{k i n}= & h(a)\left(\vec{\beta}_{1} \cdot \vec{\beta}_{1}+\vec{\beta}_{2} \cdot \vec{\beta}_{2}\right)+p(a)\left(\left(\vec{\beta}_{1} \cdot \hat{a}\right)^{2}+\left(\vec{\beta}_{2} \cdot \hat{a}\right)^{2}\right) \\
& +2 f(a) \vec{\beta}_{1} \cdot \vec{\beta}_{2}+2 g(a)\left(\vec{\beta}_{1} \cdot \hat{a}\right)\left(\vec{\beta}_{2} \cdot \hat{a}\right) .
\end{aligned}
$$

Now suppose $\vec{\beta}_{1}=\vec{\beta}_{2}=\vec{\beta}$, so that (7.6) reduces to

$$
L_{k i n}=(2 h+2 f) \beta^{2}+(2 p+2 g)(\vec{\beta} \cdot \hat{a})^{2}
$$

This configuration, however, represents the boosted solution of the two-monopole static solution. The kinetic energy should therefore be simply

$$
L_{k i n}=\frac{M_{T}}{2} \beta^{2}
$$

where $M_{T}=M_{1}+M_{2}=2 M$ is the total mass of the two-monopole solution. It then follows that the anisotropic part of (7.7) vanishes and we have

$$
\begin{aligned}
g+p & =0, \\
2(h+f) & =\frac{M_{T}}{2} .
\end{aligned}
$$

It is therefore sufficient to compute $h$ and $p$. This can be done by setting $\vec{\beta}_{1}=\vec{\beta}$ and $\vec{\beta}_{2}=0$. The kinetic Lagrangian then reduces to

$$
L_{k i n}=h(a) \beta^{2}+p(a)(\vec{\beta} \cdot \hat{a})^{2} .
$$


Suppose for simplicity also that $\vec{a}_{1}=0$ and $\vec{a}_{2}=\vec{a}$ at $t=0$. The Lagrangian density of the volume term in this case is given by

$$
\begin{aligned}
\mathcal{L}_{k i n} & =\frac{-1}{2 \kappa^{2}}\left(\frac{3 m^{3} e^{-4 \phi}}{2 r^{4}}(\vec{\beta} \cdot \vec{x})\left[\frac{\vec{\beta} \cdot \vec{x}}{r^{3}}+\frac{\vec{\beta} \cdot(\vec{x}-\vec{a})}{|\vec{x}-\vec{a}|^{3}}\right]-\frac{e^{-2 \phi} m^{2} \beta^{2}}{r^{4}}\right. \\
& \left.-\frac{e^{-4 \phi} m^{3} \beta^{2}}{2 r^{4}}\left(\frac{1}{r}+\frac{\vec{x} \cdot(\vec{x}-\vec{a})}{|\vec{x}-\vec{a}|^{3}}\right)+\frac{e^{-6 \phi} m^{4} \beta^{2}}{r^{2}}\left(\frac{1}{r^{4}}+\frac{1}{|\vec{x}-\vec{a}|^{4}}+\frac{2 \vec{x} \cdot(\vec{x}-\vec{a})}{r^{3}|\vec{x}-\vec{a}|^{3}}\right)\right) .
\end{aligned}
$$

The integration of the kinetic Lagrangian density in (7.11) over three-space yields the kinetic Lagrangian from which the metric on moduli space can be read off. For large $a$, the nontrivial leading order behaviour of the components of the metric, and hence for the functions $h(a)$ and $p(a)$, is generically of order $1 / a$. In fact, for Manton scattering of BPS monopoles, the leading order scattering angle is $2 / b[153]$, where $b$ is the impact parameter. Here we restrict our computation to the leading order metric in moduli space. A tedious but straightforward collection of $1 / a$ terms in the Lagrangian yields

$$
\frac{-1}{2 \kappa^{2}} \frac{1}{a} \int d^{3} x\left[-\frac{3 m^{4} e^{-6 \phi_{1}}}{r^{7}}(\vec{\beta} \cdot \vec{x})^{2}+\frac{m^{3} e^{-4 \phi_{1}}}{r^{4}} \beta^{2}+\frac{m^{4} e^{-6 \phi_{1}}}{r^{5}} \beta^{2}-\frac{3 m^{5} e^{-8 \phi_{1}}}{r^{6}} \beta^{2}\right],
$$

where $e^{2 \phi_{1}} \equiv 1+m / r$. The first and third terms clearly cancel after integration over three-space. The second and fourth terms are spherically symmetric. A simple integration yields

$$
\int_{0}^{\infty} r^{2} d r\left(\frac{e^{-4 \phi_{1}}}{r^{4}}-\frac{3 m^{2} e^{-8 \phi_{1}}}{r^{6}}\right)=\int_{0}^{\infty} \frac{d r}{(r+m)^{2}}-3 m^{2} \int_{0}^{\infty} \frac{d r}{(r+m)^{4}}=0 .
$$

The 1/a terms therefore cancel, and the leading order metric on moduli space is flat. This implies that to leading order the dynamical force is zero and the scattering is trivial, in agreement with the test-soliton result. In other words, there is no deviation from the initial trajectories to leading order in the impact parameter. Analogous computations for elementary strings in $D=4[21]$ and fivebranes in $D=10[160,161]$ lead to the same result of a flat metric. From $S \leftrightarrow T$ duality (see section 6.6) it follows that the metric on moduli space for solitonic strings in $D=4$ is also flat.

\subsection{Veneziano amplitude for elementary strings}

We address the scattering problem in this section from the string theoretic point of view. In particular, we calculate the string four-point amplitude for the scattering of 
macroscopic winding state strings in the infinite winding radius limit. In this scenario, we can best approximate the soliton scattering problem considered in the previous section but in the case of elementary strings in $D=4$. We find that the Veneziano amplitude obtained also indicates trivial scattering in the large winding radius limit, thus providing evidence for the identification of the elementary strings with infinitely long macroscopic fundamental strings. The fivebrane analog of this computation awaits the construction of a fundamental fivebrane theory. However, a vertex operator representation of fivebrane solitons (and also of string monopoles) should in principle be possible. The computation of the fivebrane Veneziano amplitude would then represent a dynamical test for string/fivebrane duality.

The scattering problem is set up in four dimensions, as the kinematics correspond essentially to a four dimensional scattering problem, and strings in higher dimensions generically miss each other anyway [162]. The precise compactification scheme is irrelevant to our purposes.

The winding state strings then reside in four spacetime dimensions (0123), with one of the dimensions, say $x_{3}$, taken to be periodic with period $R$, called the winding radius. The winding number $n$ describes the number of times the string wraps around the winding dimension:

$$
x_{3} \equiv x_{3}+2 \pi R n
$$

and the length of the string is given by $L=n R$. The integer $m$, called the momentum number of the winding configuration, labels the allowed momentum eigenvalues. The momentum in the winding direction is thus given by

$$
p^{3}=\frac{m}{R}
$$

The number $m$ is restricted to be an integer so that the quantum wave function $e^{i p \cdot x}$ is single valued. The total momentum of each string can be written as the sum of a right momentum and a left momentum

$$
p^{\mu}=p_{R}^{\mu}+p_{L}^{\mu}
$$

where $p_{R, L}^{\mu}=\left(E, E \vec{v}, \frac{m}{2 R} \pm n R\right), \vec{v}$ is the transverse velocity and $R$ is the winding radius. The mode expansion of the general configuration $X(\sigma, \tau)$ in the winding direction satisfying the two-dimensional wave equation and the closed string boundary conditions can 
be written as the sum of right moving pieces and left moving pieces, each with the mode expansion of an open string [83]

$$
\begin{aligned}
X(\sigma, \tau) & =X_{R}(\tau-\sigma)+X_{L}(\tau+\sigma), \\
X_{R}(\tau-\sigma) & =x_{R}+p_{R}(\tau-\sigma)+\frac{i}{2} \sum_{n=0} \frac{1}{n} \alpha_{n} e^{-2 i n(\tau-\sigma)}, \\
X_{L}(\tau+\sigma) & =x_{L}+p_{L}(\tau+\sigma)+\frac{i}{2} \sum_{n=0} \frac{1}{n} \tilde{\alpha}_{n} e^{-2 i n(\tau+\sigma)} .
\end{aligned}
$$

The right moving and left moving components are then essentially independent parts with corresponding vertex operators, number operators and Virasoro conditions.

The winding configuration described by $X(\sigma, \tau)$ describes a soliton string state. It is therefore a natural choice for us to compare the dynamics of these states with the solitonlike solutions of the previous sections (including the elementary solutions) in order to determine whether we can identify the elementary string solutions of the supergravity field equations with infinitely long fundamental strings. Accordingly, we study the scattering of the winding states in the limit of large winding radius.

Our kinematic setup is as follows. We consider the scattering of two straight macroscopic strings in the center-of-mass (CM) frame with winding number $n$ and momentum number $\pm m[83,162]$. The incoming momenta in the CM frame are given by

$$
\begin{aligned}
& p_{1 R, L}^{\mu}=\left(E, E \vec{v}, \frac{m}{2 R} \pm n R\right), \\
& p_{2 R, L}^{\mu}=\left(E,-E \vec{v},-\frac{m}{2 R} \pm n R\right) .
\end{aligned}
$$

Let $\pm m^{\prime}$ be the outgoing momentum number. For the case of $m=m^{\prime}$, the outgoing momenta are given by

$$
\begin{aligned}
& -p_{3 R, L}^{\mu}=\left(E, E \vec{w}, \frac{m}{2 R} \pm n R\right), \\
& -p_{4 R, L}^{\mu}=\left(E,-E \vec{w},-\frac{m}{2 R} \pm n R\right),
\end{aligned}
$$

where conservation of momentum and winding number have been used and where $\pm \vec{v}$ and $\pm \vec{w}$ are the incoming and outgoing velocities of the strings in the transverse $x-y$ plane. The outgoing momenta winding numbers are not a priori equal to the initial winding numbers, but must add up to $2 n$. Conservation of energy for sufficiently large $R$ then results in the above answer. This is also in keeping with the soliton scattering nature of the problem (i.e. the solitons do not change "shape" during a collision). 
For now we have assumed no longitudinal excitation $\left(m=m^{\prime}\right)$. We will later relax this condition to allow for such excitation, but show that our answer for the scattering is unaffected by this possibility. It follows from this condition that $v^{2}=w^{2}$. For simplicity we take $\vec{v}=v \hat{x}$ and $\vec{w}=v(\cos \theta \hat{x}+\sin \theta \hat{y})$, and thus reduce the problem to a two-dimensional scattering problem.

As usual, the Virasoro conditions $L_{0}=\widetilde{L}_{0}=1$ must hold, where

$$
\begin{aligned}
& L_{0}=N+\frac{1}{2}\left(p_{R}^{\mu}\right)^{2}, \\
& \widetilde{L}_{0}=\widetilde{N}+\frac{1}{2}\left(p_{L}^{\mu}\right)^{2}
\end{aligned}
$$

are the Virasoro operators [83] and where $N$ and $\widetilde{N}$ are the number operators for the rightand left-moving modes respectively:

$$
\begin{aligned}
& N=\sum \alpha_{-n}^{\mu} \alpha_{n \mu}, \\
& \tilde{N}=\sum \tilde{\alpha}_{-n}^{\mu} \tilde{\alpha}_{n \mu},
\end{aligned}
$$

where we sum over all dimensions, including the compactified ones. It follows from the Virasoro conditions that

$$
\begin{aligned}
\tilde{N}-N & =m n, \\
E^{2}\left(1-v^{2}\right) & =2 N-2+\left(\frac{m}{2 R}+n R\right)^{2} .
\end{aligned}
$$

In the following we set $n=1$ and consider for simplicity the scattering of tachyonic winding states. For our purposes, the nature of the string winding states considered is irrelevant. A similar calculation for massless bosonic strings or heterotic strings, for example, will be slightly more complicated, but will nevertheless exhibit the same essential behaviour. For tachyonic winding states we have $N=\widetilde{N}=m=0$. Equation (7.22) reduces to

$$
E^{2}\left(1-v^{2}\right)=R^{2}-2
$$

The Mandelstam variables $(s, t, u)$ are identical for right and left movers and are given by

$$
\begin{aligned}
& s=4\left[\frac{\left(R^{2}-2\right) v^{2}}{1-v^{2}}-2\right], \\
& t=-2\left[\frac{\left(R^{2}-2\right) v^{2}}{1-v^{2}}\right](1+\cos \theta), \\
& u=-2\left[\frac{\left(R^{2}-2\right) v^{2}}{1-v^{2}}\right](1-\cos \theta) .
\end{aligned}
$$


It is easy to see that $p_{i R} \cdot p_{j R}=p_{i L} \cdot p_{j L}$ holds for this configuration so that the tree level 4-point function reduces to the usual Veneziano amplitude for closed tachyonic strings [162]

$$
\begin{aligned}
A_{4} & =\frac{\kappa^{2}}{4} B(-1-s / 2,-1-t / 2,-1-u / 2) \\
& =\left(\frac{\kappa^{2}}{4}\right) \frac{\Gamma(-1-s / 2) \Gamma(-1-t / 2) \Gamma(-1-u / 2)}{\Gamma(2+s / 2) \Gamma(2+t / 2) \Gamma(2+u / 2)} .
\end{aligned}
$$

This can be seen as follows. In the standard computation of the four point function for closed string tachyons, we rely on the independence of the right and left moving open strings. For the tachyonic winding state, we also separate the right and left movers with vertex operators given by $V_{R}=e^{i p_{R} \cdot x_{R}}$ and $V_{L}=e^{i p_{L} \cdot x_{L}}$ respectively to arrive at the following expression for the amplitude

$$
A_{4}=\frac{\kappa^{2}}{4} \int d \mu_{4}(z) \prod_{i<j}\left|z_{i}-z_{j}\right|^{p_{i R} \cdot p_{j R}}\left|z_{i}-z_{j}\right|^{p_{i L} \cdot p_{j L}}
$$

From $p_{i R} \cdot p_{j R}=p_{i L} \cdot p_{j L}$, (7.26) reduces to the expression for the four-point amplitude of a nonwinding closed tachyonic string, from which the standard Veneziano amplitude in (7.25) results.

To compare the implications of $A_{4}$ with the results of section 7.1 we take $R \rightarrow \infty$. It is convenient to define $x \equiv \frac{\left(R^{2}-2\right) v^{2}}{1-v^{2}}=s / 4+2$, since the Mandelstam variables can be expressed solely in terms of $x$ and $\theta$. We now have $A_{4}=A_{4}(x, \theta)$, which can be explicitly written as

$$
A_{4}=\left(\frac{\kappa^{2}}{4}\right) \frac{\Gamma(3-2 x) \Gamma(-1+x(1+\cos \theta)) \Gamma(-1+x(1-\cos \theta))}{\Gamma(-2+2 x) \Gamma(2-x(1+\cos \theta)) \Gamma(2-x(1-\cos \theta))} .
$$

The problem reduces to studying $A_{4}$ in the limit $x \rightarrow \infty$. We now use the identity $\Gamma(1-a) \Gamma(a) \sin \pi a=\pi$ to rewrite $A_{4}$ as

$$
\begin{aligned}
A_{4}=\left(\frac{\kappa^{2}}{4 \pi}\right) & {\left[\frac{\Gamma(-1+x(1+\cos \theta)) \Gamma(-1+x(1-\cos \theta))}{\Gamma(-2+2 x)}\right]^{2} } \\
& \times\left(\frac{\sin (\pi x(1+\cos \theta)) \sin (\pi x(1-\cos \theta))}{\sin 2 \pi x}\right) .
\end{aligned}
$$

From the Stirling approximation $\Gamma(u) \sim \sqrt{2 \pi} u^{u-1 / 2} e^{-u}$ for large $u$, we obtain in the limit $x \rightarrow \infty$

$$
\begin{aligned}
A_{4} \sim & {\left[\frac{(x(1+\cos \theta))^{x(1+\cos \theta)}(x(1-\cos \theta))^{x(1-\cos \theta)}}{(2 x)^{2 x}}\right]^{2} } \\
& \times\left(\frac{\sin (\pi x(1+\cos \theta)) \sin (\pi x(1-\cos \theta))}{\sin 2 \pi x}\right)
\end{aligned}
$$


Note that the exponential terms cancel automatically. From (7.29) we notice that the powers of $x$ in the first factor also cancel. $A_{4}$ then reduces in the limit $x \rightarrow \infty$ to

$$
\begin{aligned}
A_{4} \sim\left(\frac{1+\cos \theta}{2}\right)^{2 x(1+\cos \theta)} & \left(\frac{1-\cos \theta}{2}\right)^{2 x(1-\cos \theta)} \\
& \times\left(\frac{\sin (\pi x(1+\cos \theta)) \sin (\pi x(1-\cos \theta))}{\sin 2 \pi x}\right) .
\end{aligned}
$$

The poles in the third factor in (7.30) are just the usual $s$-channel poles. It follows from (7.30) that for $\theta \neq 0, \pi A_{4} \rightarrow e^{-f(\theta) x}$ as $x \rightarrow \infty$, where $f$ is some positive definite function of $\theta$. Hence the 4-point function vanishes exponentially with the winding radius away from the poles.

In general, for finite $R$ and fixed $v$ the strings may scatter into longitudinally excited final states, i.e. states not satisfying the above assumption that $m^{\prime}=m$. The 4-point amplitude for each transition still vanishes exponentially with $R$. A simple counting argument shows that the total number of possible final states for a given $R$ is bounded by a polynomial function of $R$. This counting argument proceeds as follows:

Without loss of generality, we may assume that our incoming states have $N=\widetilde{N}=$ $m=0$ with fixed $R$ and $v$. We relax the assumption of no logitudinal excitation to obtain outgoing states with nonzero $m$. We still consider $n=1$ winding states for simplicity. Our scattering configuration can now be described by the incoming momenta

$$
\begin{aligned}
& p_{1 R, L}^{\mu}=(E, E \vec{v}, \pm R), \\
& p_{2 R, L}^{\mu}=(E,-E \vec{v}, \pm R) .
\end{aligned}
$$

and the outgoing momenta

$$
\begin{aligned}
& -p_{3 R, L}^{\mu}=\left(E_{1}, E_{1} \vec{w}_{1}, \frac{m}{2 R} \pm R\right), \\
& -p_{4 R, L}^{\mu}=\left(E_{2},-E_{2} \vec{w}_{2},-\frac{m}{2 R} \pm R\right) .
\end{aligned}
$$

Note that in general $E_{1}$ and $E_{2}$ are not equal to $E$. Without loss of generality, we take $m$ to be positive. From conservation of momentum, however, we have

$$
\begin{aligned}
E_{1}+E_{2} & =2 E, \\
E_{1} \vec{w}_{1} & =E_{2} \vec{w}_{2} .
\end{aligned}
$$


It follows from the energy momentum relations for the ingoing and outgoing momenta that

$$
\begin{aligned}
& E^{2}\left(1-v^{2}\right)=R^{2}-2, \\
& E_{1}^{2}\left(1-w_{1}^{2}\right)=2 N_{1}-2+\left(\frac{m}{2 R}+R\right)^{2}, \\
& E_{2}^{2}\left(1-w_{2}^{2}\right)=2 N_{2}-2+\left(-\frac{m}{2 R}+R\right)^{2},
\end{aligned}
$$

where $N_{1}$ and $N_{2}$ are the number operators for the the right movers of the outgoing states. Subtracting the third equation in (7.34) from the second equation and using (7.33) we obtain the relation

$$
N_{1}-N_{2}+m=\left(E_{1}-E_{2}\right) E .
$$

From the first equation in (7.34) it follows that $E$ is bounded by some multiple of $R$ for fixed $v$. It then follows from the first equation in (7.33) that both $E_{1}$ and $E_{2}$ are bounded by a multiple of $R$. So from (7.35) we see that $N_{1}-N_{2}+m$ is bounded by some quadratic polynomial in $R$. We now add the last two equations in (7.34) to obtain

$$
E_{1}^{2}\left(1-w_{1}^{2}\right)+E_{2}^{2}\left(1-w_{2}^{2}\right)=2 N_{1}+2 N_{2}+2 R^{2}+\frac{m^{2}}{2 R^{2}}-4 .
$$

The left hand side of (7.36) is clearly bounded by a quadratic polynomial in $R$. It follows that $N_{1}+N_{2}$ is also bounded by a quadratic polynomial, and that so are $N_{1}$ and $N_{2}$ and also, then, $N_{1}-N_{2}$. From the boundedness of $N_{1}-N_{2}+m$ it therefore follows that $m$ is bounded by a polynomial in $R$. Therefore the total number of possible distinct excited states (numbered by $m$ ) is bounded by a polynomial in $R$. The above argument also goes through for the case of a nonzero initial momentum number. For each transition, however, one can show that the Veneziano amplitude is dominated by an exponentially vanishing function of $R$, from a calculation entirely analogous to the zero-longitudinal excitation case worked out above. Hence the total square amplitude of the scattering (obtained by summing the square amplitudes of all possible transitions) is still dominated by a factor which vanishes exponentially with the radius, except at the poles at $\theta=0, \pi$ corresponding to forward and backward scattering, which are physically equivalent for identical bosonic strings. This is in agreement with the trivial scattering found in section 7.1 and provides further evidence for the identification of the elementary string with the fundamental string.

The above argument can be repeated for any other type of string, including the heterotic string [25]. The kinematics differ slightly from the tachyonic case but the 4-point 
function is still dominated by an exponentially vanishing factor in the large radius limit. Hence the scattering is trivial, again in agreement with the result found in section 7.1.

The Veneziano amplitude result in fact holds for arbitrary incoming winding states. A considerably more tedious calculation for the general case shows that in the large winding radius limit the outgoing strings always scatter trivially and with no change in their individual winding numbers. In this limit, then, these states scatter as true solitons [163]. It would be interesting to see if this result holds for the full quantum string loop expansion. 


\section{Exactness of solutions}

\subsection{Axionic instanton: bosonic solution}

A classical solution of string theory is considered "exact" if it satisfies the string equations of motion to all orders in the classical string parameter $\alpha^{\prime}$ (or equivalently if the Weyl anomaly coefficients of the $\sigma$-model vanish to all orders in $\alpha^{\prime}$ ). Alternatively, an exact classical solution can be demonstrated by the construction of an exact $\sigma$-model for the worldsheet of the string or by writing down the corresponding coset conformal field theory. In this section we follow the discussion of [79] to obtain an exact extension of the purely bosonic version of the axionic instanton solution of section 5.2 in the limit in which this solution reduces to a linear dilaton wormhole [164,165] (see section 2.7). Exactness is shown by combining the metric and antisymmetric tensor in a generalized curvature, which is written covariantly in terms of the tree-level dilaton field, and rescaling the dilaton order by order in the parameter $\alpha^{\prime}$. The corresponding conformal field theory is written down.

For this purpose we use the theorem of equivalence of the massless string field equations to the sigma-model Weyl invariance conditions (demonstrated to two-loop order by Metsaev and Tseytlin [166,167]), which require the Weyl anomaly coefficients $\bar{\beta}_{M N}^{G}, \bar{\beta}_{M N}^{B}$ and $\bar{\beta}^{\phi}$ to vanish identically to the appropriate order in the parameter $\alpha^{\prime}$. The two-loop solution obtained by this method suggests a representation of the sigma model as the product of a WZW [168] model and a one-dimensional CFT (a Feigin-Fuchs Coulomb gas) [78]. This representation allows us to obtain an exact solution.

The bosonic sigma model action can be written as [169]

$$
I=\frac{1}{4 \pi \alpha^{\prime}} \int d^{2} x \sqrt{-\eta}\left(\eta^{a b} \partial_{a} X^{M} \partial_{b} X^{N} g_{M N}+i \epsilon^{a b} \partial_{a} X^{M} \partial_{b} X^{N} B_{M N}+\alpha^{\prime} R^{(2)} \phi\right)
$$

where $g_{M N}$ is the sigma model metric, $\phi$ is the dilaton and $B_{M N}$ is the antisymmetric tensor and where $\eta_{a b}$ is the worldsheet metric and $R^{(2)}$ the two-dimensional curvature. The Weyl anomaly coefficients are given by $[166,167]$

$$
\begin{aligned}
\bar{\beta}_{M N}^{G} & =\beta_{M N}^{G}+2 \alpha^{\prime} \nabla_{M} \nabla_{N} \phi+\nabla_{(M} W_{N)}, \\
\bar{\beta}_{M N}^{B} & =\beta_{M N}^{B}+\alpha^{\prime} H_{M N}{ }^{P} \partial_{P} \phi+\frac{1}{2} H_{M N}{ }^{P} W_{P}, \\
\bar{\beta}^{\phi} & =\beta^{\Phi}+\alpha^{\prime}(\partial \phi)^{2}+\frac{1}{2} W^{M} \partial_{M} \phi,
\end{aligned}
$$


where $\beta_{M N}^{G}, \beta_{M N}^{B}$ and $\beta^{\Phi}$ are the RG $\beta$ functions and where $H_{M N P}=\partial_{[M} B_{N P]}$ and $W_{M}=-\left(\alpha^{\prime 2} / 24\right) \nabla_{M} H^{2}$.

We follow Metsaev and Tseytlin's computation of the renormalization group beta functions for the general sigma-model and combine dimensional regularization and the minimal subtraction scheme with the following generalized prescription for contraction of $\epsilon^{a b}$ tensors [166]:

$$
\epsilon^{a b} \epsilon^{c d}=f(d)\left(\delta^{a c} \delta^{b d}-\delta^{a d} \delta^{b c}\right)
$$

where $f(d)=1-f_{1} \epsilon+O\left(\epsilon^{2}\right)$ and $\epsilon=d-2$. We note that the precise form of the renormalization group beta functions at two-loop order is not scheme-independent but depends on the choice of $f_{1}$. Here we set $f_{1}=-1$, for which Metsaev and Tseytlin obtain the following two-loop expressions for the Weyl anomaly coefficients $[166,167]$ :

$$
\begin{aligned}
& \bar{\beta}_{M N}^{G}=\alpha^{\prime}\left(\hat{R}_{(M N)}+2 \nabla_{M} \nabla_{N} \phi\right)
\end{aligned}
$$

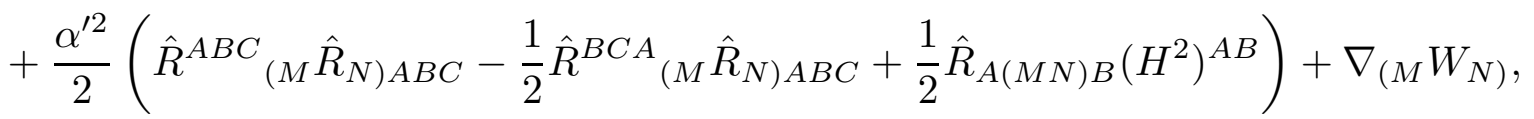

$$
\begin{aligned}
& \bar{\beta}_{M N}^{B}=\alpha^{\prime}\left(\hat{R}_{[M N]}+H_{M N}^{P} \partial_{P} \phi\right) \\
& +\frac{\alpha^{\prime 2}}{2}\left(\hat{R}_{[M}^{A B C} \hat{R}_{N]) A B C}-\frac{1}{2} \hat{R}_{[M C A}^{B \hat{R}_{N] A B C}}+\frac{1}{2} \hat{R}_{A[M N] B}\left(H^{2}\right)^{A B}\right)+\frac{1}{2} H_{M N}{ }^{P} W_{P}, \\
& \bar{\beta}^{\phi}=\frac{D}{6}-\frac{\alpha^{\prime}}{2}\left(\nabla^{2} \phi-2(\partial \phi)^{2}+\frac{1}{12} H^{2}\right) \\
& +\frac{\alpha^{\prime 2}}{16}\left(2\left(H^{2}\right)^{M N} \nabla_{M} \nabla_{N} \phi+R_{P M N K}^{2}-\frac{11}{2} R H H+\frac{5}{24} H^{4}+\frac{11}{8}\left(H_{M N}^{2}\right)^{2}+\frac{4}{3} \nabla H \cdot \nabla H\right) \\
& +\frac{1}{2} W^{M} \partial_{M} \phi,
\end{aligned}
$$

where $\nabla H \cdot \nabla H \equiv \nabla_{M} H_{N P Q} \nabla^{M} H^{N P Q}$. Unless otherwise indicated, all expressions are written to two loop order in the beta-functions, which corresponds to $O\left(\alpha^{\prime}\right)$ in the effective action (3.81). Also, the only nontrivial coefficients occur when all indices are in the transverse curved four space, as it is clear that the flat dimensions do not contribute, so henceforth we restrict ourselves to this space.

It follows from section 5.2 and the abovementioned theorem of equivalence that any dilaton function satisfying $e^{-2 \phi} \square e^{2 \phi}=0$ with

$$
\begin{aligned}
g_{\mu \nu} & =e^{2 \phi} \delta_{m n} \quad m, n=1,2,3,4, \\
g_{\mu \nu} & =\eta_{\mu \nu} \quad \mu, \nu=5, \ldots, 26, \\
H_{m n p} & = \pm 2 \epsilon_{m n p k} \partial^{k} \phi \quad m, n, p, k=1,2,3,4
\end{aligned}
$$


is an $O\left(\alpha^{\prime}\right)$ solution to (8.4). Note that this is essentially the fivebrane ansatz, except that in bosonic string theory we have 22 flat directions instead of 6. Eqs. (5.13) and (5.14) follow immediately as in the supersymmetric case of section 5.2.

We now specialize to the spherically symmetric case of $e^{2 \phi}=Q / r^{2}$ in (8.5) and determine the $O\left(\alpha^{\prime}\right)$ corrections to the massless fields in (8.5) so that the Weyl anomaly coefficients vanish to $O\left(\alpha^{2}\right)$. For this solution we notice [79]

$$
\nabla_{m} \nabla_{n} \phi=0
$$

and therefore from (5.13)

$$
\hat{R}_{j k l}^{i}=0,
$$

and we have what is called a "parallelizable" space $[166,167]$. To maintain a parallelizable space to $O\left(\alpha^{\prime}\right)$ we keep $g_{\mu \nu}$ and $H_{\alpha \beta \gamma}$ in their lowest order form and assume that any corrections to (8.5) appear in the dilaton:

$$
\begin{aligned}
\phi & =\bar{\phi}+\alpha^{\prime} \phi_{1}+\ldots \\
e^{2 \bar{\phi}} & =\frac{Q}{r^{2}}, \\
g_{\mu \nu} & =e^{2 \bar{\phi}} \delta_{m n}, \\
H_{m n p} & = \pm 2 \epsilon_{m n p k} \partial^{k} \bar{\phi} .
\end{aligned}
$$

It follows from (8.8) that $H^{2}=24(\partial \bar{\phi})^{2}=24 / Q$ and thus $W_{m}=0$. It follows from (8.7) that $\bar{\beta}_{M N}^{G}$ and $\bar{\beta}_{M N}^{B}$ vanish identically to two loop order and that

$$
\begin{aligned}
\bar{\beta}^{\phi}=\frac{D}{6}+ & \alpha^{\prime} \\
& \left((\partial \phi)^{2}-\frac{1}{Q}\right) \\
& +\frac{\alpha^{\prime 2}}{16}\left(R_{k m n p}^{2}-\frac{11}{2} R H H+\frac{5}{24} H^{4}+\frac{11}{8}\left(H_{m n}^{2}\right)^{2}+\frac{4}{3} \nabla H \cdot \nabla H\right) .
\end{aligned}
$$

We use the relations in equation (34) in [166] for parallelizable spaces and the observation that $\left(H_{m n}^{2}\right)^{2}=2 H^{4}=192 / Q^{2}$ for our solution to get the identities

$$
\begin{aligned}
R_{k m n p}^{2} & =\frac{1}{8} H^{4}, \\
R H H & =\frac{1}{2} H^{4}, \\
\nabla H \cdot \nabla H & =0 .
\end{aligned}
$$


(8.9) then simplifies further to

$$
\bar{\beta}^{\phi}=\frac{D}{6}+\alpha^{\prime}\left((\partial \phi)^{2}-\frac{1}{Q}\right)+2 \frac{\alpha^{\prime 2}}{Q^{2}} .
$$

The lowest order term in $\bar{\beta}^{\phi}$ is proportional to the central charge and the $O\left(\alpha^{\prime}\right)$ terms vanish identically. With the choice $\vec{\nabla} \phi_{1}=-(1 / Q) \vec{\nabla} \phi_{0}$, the $O\left(\alpha^{2}\right)$ terms also vanish identically. The two-loop solution is then given by

$$
\begin{aligned}
e^{2 \phi} & =\frac{Q}{r^{2\left(1-\frac{\alpha^{\prime}}{Q}\right)},} \\
g_{\mu \nu} & =\frac{Q}{r^{2}} \delta_{m n}, \\
H_{m n p} & = \pm 2 \epsilon_{m n p k} \partial^{k} \phi_{0},
\end{aligned}
$$

which corresponds to a simple rescaling of the dilaton. A quick check shows that this solution has finite action near the singularity.

We now rewrite $\bar{\beta}^{\phi}$ in (8.11) in the following suggestive form:

$$
\begin{aligned}
6 \bar{\beta}^{\phi} & =\left(1+6 \alpha^{\prime}(\partial \phi)^{2}\right)+\left(3-6 \frac{\alpha^{\prime}}{Q}+12\left(\frac{\alpha^{\prime}}{Q}\right)^{2}\right) \\
& =4 .
\end{aligned}
$$

The above splitting of the central charge $c=6 \bar{\beta}^{\phi}$ suggests the decomposition of the corresponding sigma model into the product of a one-dimensional CFT (a Feigin-Fuchs Coulomb gas) and a three-dimensional WZW model with an $S U(2)$ group manifold [166,167]. This can be seen as follows. Setting $u=\ln r$, we can rewrite (8.1) for our solution [112] in the form $I=I_{1}+I_{3}$, where

$$
I_{1}=\frac{1}{4 \pi \alpha^{\prime}} \int d^{2} x\left(Q(\partial u)^{2}+\alpha^{\prime} R^{(2)} \phi\right)
$$

is the action for a Feigin-Fuchs Coulomb gas, which is a one-dimensional CFT with central charge given by $c_{1}=1+6 \alpha^{\prime}(\partial \phi)^{2}[170]$. The imaginary charge of the Feigin-Fuchs Coulomb gas describes the dilaton background growing linearly in imaginary time and $I_{3}$ is the Wess-Zumino-Witten [168] action on an $S U(2)$ group manifold with central charge

$$
c_{3}=\frac{3 k}{k+2} \simeq 3-\frac{6}{k}+\frac{12}{k^{2}}+\ldots
$$


where $k=Q / \alpha^{\prime}$, called the "level" of the WZW model, is an integer. This can be seen from the quantization condition on the Wess-Zumino term [168]

$$
\begin{aligned}
I_{W Z} & =\frac{i}{4 \pi \alpha^{\prime}} \int_{\partial S_{3}^{ \pm}} d^{2} x \epsilon^{a b} \partial_{a} x^{m} \partial_{b} x^{n} B_{m n} \\
& =\frac{i}{12 \pi \alpha^{\prime}} \int_{S_{3}^{ \pm}} d^{3} x \epsilon^{a b c} \partial_{a} x^{m} \partial_{b} x^{n} \partial_{c} x^{p} H_{m n p} \\
& =2 \pi i\left(\frac{Q}{\alpha^{\prime}}\right) .
\end{aligned}
$$

Thus $Q$ is not arbitrary, but is quantized in units of $\alpha^{\prime}$. This quantization condition is equivalent to $(2.53)$ once we set $Q=k_{6}$ from (2.51).

We use this splitting to obtain exact expressions for the fields by fixing the metric and antisymmetric tensor field in their lowest order form and rescaling the dilaton order by order in $\alpha^{\prime}$. The resulting expression for the dilaton is

$$
e^{2 \phi}=\frac{Q}{r \sqrt{\frac{4}{1+\frac{2 \alpha^{\prime}}{Q}}}} .
$$

\subsection{Symmetric fivebrane}

The symmetric fivebrane of section 5.3 was obtained by equating the curvature of the Yang-Mills gauge field with the generalized curvature of the axionic instanton. This solution thus represents a supersymmetric extension of the bosonic solution of section 8.1. Unlike the bosonic solution, however, the symmetric fivebrane can be argued to be an exact classical solution in the multi-instanton case, and not merely in the wormhole limit $[32,33]$. In that particular limit, however, one can demonstrate exactness of the symmetric fivebrane without modification to the dilaton from both the $\beta$-function and CFT points of view. Furthermore, these exactness arguments can be supplemented by superconformal worldsheet $\sigma$-model arguments and nonrenormalization theorems due to higher worldsheet supersymmetry.

From the $\beta$-function (or equations of motion) point of view, one would guess that the tree-level symmetric fivebrane solution is exact since $A_{M}=\Omega_{ \pm M}$ suggests that all the higher order corrections vanish. This can be seen by noting that the higher order corrections to the effective action (up to at least $O\left(\alpha^{\prime 3}\right)$ ) are all at least linear in the tensor $[171,172])$

$$
T_{A B C D} \equiv \operatorname{tr} \hat{R}_{[A B} \hat{R}_{C D]}-\operatorname{tr} F_{[A B} F_{C D]}
$$


which clearly vanishes since the two curvatures are identical.

The fact that the single instanton solution in the heterotic case carries through even to $O\left(\alpha^{\prime}\right)$ without correction to the dilaton seems to contradict the bosonic solution in the wormhole limit by suggesting that the expansion for the Weyl anomaly coefficient $\bar{\beta}^{\phi}$ terminates at one loop. This seeming contradiction is resolved by noting that in the heterotic case the gauge rotation which decouples the fermions is anomalous [36], and the effect of this anomaly is to replace $k$ in (8.15) by $k^{\prime}=k-2$ [173]. The bosonic contribution to this part of the central charge is then given by

$$
c_{3}=\frac{3 k^{\prime}}{k^{\prime}+2}=3-\frac{6}{k}=3-\frac{6 \alpha^{\prime}}{Q},
$$

which indeed terminates at one loop order. The exactness of the splitting then requires that $c_{1}$ not get any corrections from $(\partial \phi)^{2}$ so that $c_{1}+c_{3}=4$ is exact for the tree-level value of the dilaton [32]. In [32,36], arguments based on $N=4$ worldsheet supersymmetry were presented to support the claim of exactness of the heterotic fivebrane. We summarize these arguments below.

To show that a solution is an exact solution in string theory one must in principle show that the solution derives form a superconformal worldsheet sigma-model. For the heterotic fivebrane, one can show that the corresponding $\sigma$-model possesses $(4,4)$ worldsheet supersymmetry, in which case nonrenormalization theorems show that the tree-level solution is exact.

For generic fivebrane solutions annihilated by $D=6, N=1$ spacetime supersymmetries, the compactification from 10 to 6 dimensions maintains $N=1$ spacetime supersymmetry. In compactification from 10 to 4 dimensions with $N=1$ spacetime supersymmetry, the compactified 6 dimensions must possess at least $(2,0)$ supersymmetry. Similarly, in compactifying to $D=4, N=2$, the six-dimensional compactification sigma-model must possess at least $(4,0)$ supersymmetry. Since $N=1, D=6$ spacetime supersymmetry is equivalent to $N=2, D=4$ spacetime supersymmetry, a generic fivebrane solution must possess at least $(4,0)$ spacetime supersymmetry.

In writing down a generic heterotic fivebrane sigma-model action with $(1,0)$ worldsheet supersymmetry, the only worldsheet fermions that couple nontrivially are four right movers which couple to the generalized connection $\Omega_{-}$and four left movers which couple to the gauge field, which must be an instanton to satisfy the tree-level supersymmetry equations (see section 5.3). Now if we demand that the dilaton satisfy $e^{-2 \phi} \square e^{2 \phi}=0$ and consequently 
the exactness condition $A=\Omega_{+}$, the theory becomes left-right symmetric and thus must possess at least $(1,1)$ worldsheet supersymmetry. This can also be seen by observing that for $d H=0$

$$
R\left(\Omega_{+}\right)_{m n p q}=R\left(\Omega_{-}\right)_{p q m n}
$$

follows immediately from (5.13). The symmetric fivebrane therefore possesses $(4,4)$ worldsheet supersymmetry. For the explicit construction of the $(4,4)$ worldsheet $\sigma$-model, we refer the reader to [36], in which it is argued that the existence of more than one pair of complex structures elevates the worldsheet supersymmetry to $(4,4)$. As a consequence of $(4,4)$ supersymmetry, the theory is finite and from nonrenormalization theorems the tree-

level solution is exact. In a similar manner, it can be argued that all symmetric solutions correspond to $(4,4)$ supersymmetry on the worldsheet of the fundamental string and are thus presumably exact to all orders in $\alpha^{\prime}$. In particular, this includes the $D=4$ monopole, string and domain wall solutions of section 6.5 .

From algebraic conformal field theory constructions, $(4,4)$ worldsheet supersymmetry manifests itself in the wormhole limit in the form of two $S U(2)$ Kac-Moody symmetries, one from the $N=4$ superalgebra and the other from the WZW wormhole throat $[174,175,36]$.

The double-instanton string solution of section 5.4 can also be argued to be exact using similar arguments as above. Both the $\beta$-function arguments and the $(4,4)$ worldsheet supersymmetry arguments above can be used in essentially the same manner to demonstrate exactness of the string solution. The explicit construction in this case can be found in $[176]$.

\subsection{Exact elementary string}

Finally, we summarize the arguments of $[177,178]$ regarding the exactness of the elementary string. Consider the family of backgrounds with metric and antisymmetric tensor characterized by a single function $F(x)$ and dilaton $\phi(x)$ :

$$
d s^{2}=F(x) \eta_{\mu \nu} d x^{\mu} d x^{\nu}+d x_{i} d x^{i}, \quad B_{01}=\frac{1}{2} F(x)
$$

The two functions $F$ and $\phi$ depend only on the transverse coordinates $x^{i}$. The leading order string equations of (8.1) then reduce to [179]

$$
\partial^{2} F^{-1}=2 b^{i} \partial_{i} F^{-1}, \quad \phi=\phi_{0}+b_{i} x^{i}+\frac{1}{2} \ln F(x)
$$


where $b_{i}$ is a constant vector. It was argued in $[179,177]$ that these solutions should not receive non-trivial higher order corrections, and that one can show [177] that there exists a scheme in which all of these solutions are exact and receive no $\alpha^{\prime}$ corrections. Since the equation for $F^{-1}$ is linear, linear combinations of these solutions yield new exact solutions. For $b_{i}=0$ one recovers the elementary string solution of [19]:

$$
F^{-1}=1+\frac{M}{r^{D-4}}, \quad D>4 ; \quad F^{-1}=1-M \ln r, \quad D=4, \quad r^{2}=x_{i} x^{i}
$$

where $D$ is the number of spacetime dimensions. The case $D=10$ was discussed in section 2.1. The exactness arguments proceed as follows.

For a more general model with curved transverse space, defined as [177]

$$
L_{F}=F(x) \eta_{\mu \nu} \partial x^{\mu} \bar{\partial} x^{\nu}+\left(G_{i j}+B_{i j}\right)(x) \partial x^{i} \bar{\partial} x^{j}+\alpha^{\prime} \mathcal{R} \phi(x)
$$

the all-order conformal invariance conditions are satisfied [177] provided one is given a conformal "transverse" theory $\left(G^{\prime}, B^{\prime}, \phi^{\prime}\right)$ and

$$
G_{i j}=G_{i j}^{\prime}+\frac{1}{2} \alpha^{\prime} \partial_{i} \ln F \partial_{j} \ln F, \quad \phi=\phi^{\prime}+\frac{1}{2} \ln F, \quad B_{i j}=B_{i j}^{\prime},
$$

with $F$ satisfying

$$
-\omega^{\prime} F^{-1}+\partial^{i} \phi^{\prime} \partial_{i} F^{-1}=-\frac{1}{2} \nabla^{2} F^{-1}+O\left(\alpha^{\prime}\right)+\partial^{i} \phi^{\prime} \partial_{i} F^{-1}=0 .
$$

Here $\omega^{\prime}$ is the anomalous dimension operator depending on $G^{\prime}[177,178]$. When $\left(G^{\prime}, B, \phi^{\prime}\right)$ correspond to a known CFT this equation can be written down explicitly to all orders in $\alpha^{\prime}$. Since the relation between $G$ and $G^{\prime}$ is local and the transverse theory is, in general, defined modulo local coupling redefinitions, one can argue [177] that there exists a ("leading-order") scheme in which the exact solution is represented by the conformal transverse theory $\left(G, B, \phi^{\prime}\right)$ and $F$ satisfying (8.26).

The simplest example of the conformal transverse model is flat space with a linear dilaton, in which the corresponding model is represented (in the leading-order scheme) by

$$
G_{i j}=\delta_{i j}, \quad \phi=\phi_{0}+b_{i} x^{i}+\frac{1}{2} \ln F
$$

In this scheme, the exact form of the equation for the function $F$ is simply

$$
-\frac{1}{2} \partial^{2} F^{-1}+b^{i} \partial_{i} F^{-1}=0
$$


i.e. the model

$$
L_{F}=F(x) \eta_{\mu \nu} \partial x^{\mu} \bar{\partial} x^{\nu}+\partial x^{i} \bar{\partial} x_{i}+\alpha^{\prime} \mathcal{R}\left(\phi_{0}+b_{i} x^{i}+\frac{1}{2} \ln F\right),
$$

with $F$ satisfying (8.28) is conformally invariant to all orders, i.e. gives an exact string solution.

In this scheme the leading-order duality is exact since the leading-order dual to (8.29) is the model

$$
L_{K}=\eta_{\mu \nu} \partial x^{\mu} \bar{\partial} x^{\nu}+F^{-1}(x) \partial x^{i} \bar{\partial} x_{i}+\alpha^{\prime} \mathcal{R}\left(\phi_{0}+b_{i} x^{i}\right),
$$

which represents an exact solution [177] if $F$ solves (8.28). In particular, one concludes that there exists a scheme in which the elementary solution (8.23) is a classical string solution to all orders in $\alpha^{\prime}$. 


\section{Recent developments}

This report summarizes the status of string solitons up to the summer of '94. Since the subject is developing so rapidly it is difficult to know when to draw the line. Here we record some of the most interesting developments:

1) Seiberg and Witten [180], and Vafa and Witten [181] have provided further evidence for the $S$-duality of section 6.2 in global supersymmetric Yang-Mills theories. An interesting attempt to show evidence for $S$-duality in string theory can be found in the recent paper of Gauntlett and Harvey [182].

2) Frampton and Kephart [183] have suggested that the identification of Bogomol'nyi string states with extreme black holes of section 6.8 be generalized and claim that all massive string states are black holes. As discussed in section 6.8, we remain agnostic on this point.

3) The entropy of a scalar/Maxwell parameter $a$ is given by [136]

$$
S=\pi r_{+}^{2}\left(\frac{r_{+}-r_{-}}{r_{+}}\right)^{\frac{2 a^{2}}{1+a^{2}}}
$$

and so the claim of section 6.8 that the extreme $\left(r_{+}=r_{-}\right)$black holes have zero entropy, while valid for $a \neq 0$, is ambiguous for $a=0$. This ambiguity is resolved in recent papers by Hawking, Horowitz and Ross [184] and Gibbons and Kallosh [185].

4) Both the $a=\sqrt{3}$ and $a=1$ supersymmetric black holes, shown to be solutions of the heterotic string in $[144,125]$ and discussed in sections 6.7 and 6.8 have recently been shown to be exact to all orders in $\alpha^{\prime}$ by Horowitz and Tseytlin [186] (at least in the context of the bosonic string). Further discussions of black holes, supersymmetry and duality may be found in the papers by Cvetič and Youm [187] and by Bergshoeff, Kallosh and Ortin [188] (for an earlier reference, see Kalara and Nanopoulos [189]).

5) The conjecture that $S$ and $T$ duality be united into $O(8,24 ; Z)$, as discussed in section 6.8 , has recently been taken up by Sen [190] in the context of the $D=3$ heterotic string. He shows that the fundamental string is related to the stringy cosmic string [119] by an $O(8,24 ; Z)$ transformation. In [48], Duff, Ferrara, Khuri and Rahmfeld find an $O(8,24 ; Z)$ transformation relating the fundamental string to the dual string [99], and 
make various connections between the $T$-duality group and spacetime supersymmetry. See also the paper by Maharana [191].

6) On the issue of $p$-brane singularities, Gibbons, Horowitz and Townsend [192] have considered further the higher-dimensional resolution of dilaton black hole singularities [46] discussed in sections 3.7 and 6.8. They conclude that an $a=1 / \sqrt{3}$ black hole in $D=4$, the self-dual string in $D=6$ [49], the self-dual threebrane in $D=10[50,58]$ and the fivebrane in $D=11$ [51] are completely nonsingular.

7) Hull and Townsend [193] have generalized the arguments of Duff and Rahmfeld [116] and of section 6.8 concerning string states as black holes to the Type II superstring, where the four-dimensional supersymmetry is $N=8$ and where the duality group $E_{7(7)}(Z)$ contains $O(6,6 ; Z)$ and $S L(2, Z)$ as subgroups [117]. This leads to a new and interesting interplay between elementary and solitonic string states not found in the $D=4$ heterotic string. They also make the interesting observation that the flatness of the moduli space for solitons that break half the supersymmetry is protected by supersymmetry for $N=8$ supergravity but not for $N=4$. Thus there is no apparent reason to expect the moduli space metric of extreme black hole solitons of the exact heterotic string theory (to all orders in $\alpha^{\prime}$ and $g$ ) to be flat. They even make the bolder suggestion that when solitons are taken into account there is no distinction between the field theory and the string theory.

There is now a consensus that the really interesting questions of superstring theory will never be answered within the framework of a weak coupling perturbation expansion. It is thus refreshing to see this new burst of activity in non-perturbative string theory. 


\section{Acknowledgements}

We have enjoyed useful conversations with Atish Dabholkar, John Dixon, Sergio Ferrara, Gary Gibbons, Chris Hull, Jim Liu, Nick Manton, Ruben Minasian, Chris Pope, Joachim Rahmfeld, John Schwarz, Ashoke Sen, Ergin Sezgin and Paul Townsend. MJD is grateful to the Theory Group, Rutherford-Appleton Laboratory; the CERN Theory Division; the Director and Staff of the Isaac Newton Institute, and the organizers of the Topological Defects programme, for their hospitality. RRK is grateful to the Princeton University Physics Department for its hospitality. 


\section{References}

[1] S. Coleman, "Classical Lumps and Their Quantum Descendants", in New Phenomena in Subnuclear Physics, ed A. Zichichi (Plenum, New York, 1976).

[2] R. Jackiw, Rev. Mod. Phys. 49 (1977) 681.

[3] R. Rajaraman, Solitons and Instantons (North-Holland, Amsterdam, 1982).

[4] A. Vilenkin and E. P. Shellard, Cosmic String and Other Topological Defects, (Cambridge University Press, 1994).

[5] M. B. Hindmarsh and T. W. B. Kibble, SUSX-TP-94-74, IMPERIAL/TP/94-95/5, NI 94025, hepph/9411342.

[6] W. Siegel, Phys. Lett. B128 (1983) 397.

[7] M. B. Green and J. Schwarz, Phys. Lett. B136 (1984) 367.

[8] J. Hughes, J. Liu and J. Polchinski, Phys. Lett. B180 (1986) 370.

[9] E. Bergshoeff, E. Sezgin and P. K. Townsend, Ann. Phys. 199 (1990) 340.

[10] A. Achucarro, J. Evans, P. K. Townsend and D. Wiltshire, Phys. Lett. B198 (1987) 441.

[11] A. H. Chamseddine, Phys. Rev. D24 (1981) 3065.

[12] E. A. Bergshoeff, M. de Roo, B. de Wit and P. van Nieuwenhuizen, Nucl. Phys. B195 (1982) 97

[13] G. F. Chapline and N. S. Manton, Phys. Lett. B120 (1983) 105.

[14] M. Green and J. Schwarz, Phys. Lett. B149 (1984) 117.

[15] A. Salam and E. Sezgin, Supergravities in Diverse Dimensions, (North Holland/World Scientific, 1989).

[16] S. J. Gates and H. Nishino, Phys. Lett. B173 (1986) 52.

[17] M. J. Duff, Class. Quantum Grav. 5 (1988) 189.

[18] P. K. Townsend, Phys. Lett. B202 (1988) 53.

[19] A. Dabholkar, G. W. Gibbons, J. A. Harvey and F. Ruiz Ruiz, Nucl. Phys. B340 (1990) 33.

[20] C. G. Callan and R. R. Khuri, Phys. Lett. B261 (1991) 363.

[21] R. R. Khuri, Phys. Lett. 307 (1993) 302.

[22] R. R. Khuri, Nucl. Phys. B403 (1993) 335.

[23] M. J. Duff and K. Stelle, Phys. Lett. B253 (1991) 113.

[24] A. Strominger, Nucl. Phys. B343 (1990) 167.

[25] D. J. Gross, J. A. Harvey, E. Martinec and R. Rohm, Nucl. Phys. B256 (1985) 253.

[26] M. J. Duff and J. X. Lu, Nucl. Phys. B354 (1991) 129.

[27] R. I. Nepomechie, Phys. Rev. D31 (1984) 1921.

[28] C. Teitelboim, Phys. Lett. B167 (1986) 69.

[29] M. J. Duff and J. X. Lu, Nucl. Phys. B354 (1991) 141. 
[30] M. J. Duff and J. X. Lu, Phys. Rev. Lett. 66 (1991) 1402.

[31] M. J. Duff and J. X. Lu, Nucl. Phys. B357 (1991) 534.

[32] C. G. Callan, J. A. Harvey and A. Strominger, Nucl. Phys. B359 (1991) 611.

[33] C. G. Callan, J. A. Harvey and A. Strominger, Nucl. Phys. B367 (1991) 60.

[34] M. J. Duff, R. R. Khuri and J. X. Lu, Nucl. Phys. B377 (1992) 281.

[35] J. Strathdee, Int. J. Mod. Phys. A2 (1987) 273.

[36] C. Callan, J. Harvey and A. Strominger, Supersymmetric String Solitons, Lectures given at Trieste Summer School, Trieste, Italy, 1991.

[37] E. Witten and D. Olive, Phys. Lett. B78 (1978) 97.

[38] M. K. Prasad and C. M. Sommerfield, Phys. Rev. Lett. 35 (1975) 760.

[39] E. Corrigan and P. Goddard, Commun. Math. Phys. 80 (1981) 575.

[40] N. S. Manton, Nucl. Phys. B126 (1977) 525.

[41] P. Goddard, J. Nuyts and D. Olive, Nucl. Phys. B125 (1977) 1.

[42] C. Montonen and D. Olive, Phys. Lett. B72 (1977) 117.

[43] H. Osborn, Phys. Lett. B83 (1979) 321.

[44] E. Bergshoeff, M. J. Duff, C. N. Pope and E. Sezgin, Phys. Lett. B199 (1987) 69.

[45] G.W. Gibbons and P.K. Townsend, Phys. Rev. Lett. 71 (1993) 3754.

[46] M.J. Duff, G.W. Gibbons and P.K. Townsend Phys. Lett. B (1994)

[47] M.J. Duff, P.K. Townsend and P. van Nieuwenhuizen, Phys. Lett. B122 (1983) 232.

[48] M. J. Duff, S. Ferrara, R. R. Khuri and J. Rahmfeld, in preparation.

[49] M. J. Duff and J. X. Lu, Nucl. Phys. B416 (1994) 301.

[50] G. Horowitz and A. Strominger, Nucl. Phys. B360 (1991) 197.

[51] R. Güven, Phys. Lett. B276 (1992) 49.

[52] G. W. Gibbons, Nucl. Phys. B207 (1982) 337.

[53] P. Dobiasch and D. Maison, Gen. Rel. Grav. 14 (1982) 231.

[54] A. Chodos and S. Detweiler, Gen. Rel. Grav. 14 (1982) 879.

[55] D. Pollard, J. Phys. A16 (1983) 565.

[56] G. W. Gibbons and K. Maeda, Nucl. Phys. B298 (1988) 741.

[57] L. Romans, Nucl. Phys. B276 (1986) 71.

[58] M. J. Duff and J. X. Lu, Phys. Lett. B273 (1991) 409.

[59] J. X. Lu, Phys. Lett. B313 (1993) 29.

[60] R. Gregory and R. Laflamme, Phys. Rev. Lett. 70 (1993) 2837.

[61] M. J. Duff and J. X. Lu, Nucl. Phys. B390 (1993) 276.

[62] H. Nicolai, P. K. Townsend and P. van Nieuwenhuizen, Lett. Nuovo Cimento 30 (1981) 315.

[63] P. A. M. Dirac, Pro. R. Soc. A133 (1931) 60.

[64] D. Zwanziger, Phys. Rev. 176 (1968) 1480, 1489.

[65] J. Schwinger, Phys. Rev. 144 (1966) 1087; 173 (1968) 1536. 
[66] E. Witten, Phys. Lett. B86 (1979) 283.

[67] R. R. Khuri, Phys. Rev. D48 (1993) 2947.

[68] J. Harvey and A. Strominger, Phys. Rev. Lett. 66 (1991) 549.

[69] R. Güven, Phys. Lett. B212 (1988) 277.

[70] M. J. Duff, P. S. Howe, T. Inami and K. Stelle, Phys. Lett. B191 (1987) 70.

[71] P. Candelas, G. T. Horowitz, A. Strominger and E. Witten, Nucl. Phys. B258 (1984) 46.

[72] J. A. Dixon, M. J. Duff and J. C. Plefka, Phys. Rev. Lett. 69 (1992) 3009.

[73] P. K. Townsend, in Proceedings of the 13th GIFT Seminar on Theoretical Physics: Recent Problems in Mathematical Physics Salamanca, Spain, 15-27 June, 1992.

[74] G. 't Hooft, Phys. Rev. Lett. 37 (1976) 8.

[75] F. Wilczek, in Quark confinement and field theory, Eds. D. Stump and D. Weingarten, (John Wiley and Sons, New York, 1977).

[76] E. F. Corrigan and D. B. Fairlie, Phys. Lett. B67 (1977) 69.

[77] R. Jackiw, C. Nohl and C. Rebbi, Phys. Rev. D15 (1977) 1642.

[78] S. J. Rey, in Proceedings of Tuscaloosa Workshop on Particle Physics, (Tuscaloosa, Alabama, 1989).

[79] R. R. Khuri, Phys. Lett. B259 (1991) 261.

[80] R. R. Khuri, Phys. Rev. D46 (1992) 4526.

[81] C. W. Bernard, N. H. Christ, A. H. Guth and E. J. Weinberg, Phys. Rev. D16 (1977) 2967.

[82] J. M. Charap and M. J. Duff, Phys. Lett. B69 (1977) 445.

[83] M. Green, J. Schwarz and E. Witten, Superstring theory (Cambridge University Press, 1987).

[84] J. Ellis, P. Jetzer and L. Mizrachi, Nucl. Phys. B303 (1988) 1.

[85] J. Dixon, M. J. Duff and E. Sezgin, Phys. Lett. B279 (1992) 265.

[86] M. J. Duff and R. Minasian, CTP-TAMU-16/94, hepth/9406198.

[87] D. J. Gross and J. Sloan, Nucl. Phys. B291 (1987) 41.

[88] B. Grossman, T. W. Kephart and J. D. Stasheff, Phys. Lett. B220 (1989) 431.

[89] J. X. Lu, Supersymmetric Extended Objects, Ph.D. Thesis, Texas A\&M University (1992), UMI 53 08B, Feb. 1993.

[90] D. B. Fairlie and J. Nuyts, J. Phys. A17 (1984) 2867.

[91] S. Fubini and H. Nicolai, Phys. Lett. B155 (1985) 369

[92] M. J. Duff, T. Inami, C. N. Pope, E. Sezgin and K. S. Stelle, Nucl. Phys. B297 (1988) 515.

[93] K. Fujikawa and J. Kubo, Nucl. Phys. B356 (1991) 208.

[94] R. Rohm and E. Witten, Ann. Phys. 170 (1986) 454.

[95] T. Banks, M. Dine, H. Dijkstra and W. Fischler, Phys. Lett. B212 (1988) 45. 
[96] R. R. Khuri, Phys. Lett. B294 (1992) 325.

[97] R. R. Khuri, Nucl. Phys. B387 (1992) 315.

[98] J. Gauntlett, J. Harvey and J. T. Liu, Nucl. Phys. B409 (1993) 363.

[99] M. J. Duff and R. R. Khuri, Nucl. Phys. B411 (1994) 473.

[100] A. Sen, Int. J. Mod. Phys. A8 (1993) 5079.

[101] J. H. Schwarz and A. Sen, Nucl. Phys. B411 (1994) 35.

[102] P. Binétruy, Phys. Lett. B315 (1993) 80.

[103] S. Ferrara, C. Kounnas and M. Porrati, Phys. Lett. B181 (1986) 263.

[104] M. Terentev, Sov. J. Nucl. Phys. 49 (1989) 713.

[105] S. F. Hassan and A. Sen, Nucl. Phys. B375 (1992) 103.

[106] J. Maharana and J. Schwarz, Nucl. Phys. B390 (1993) 3.

[107] A. Sen, Int. J. Mod. Phys. A9 (1994) 3707.

[108] A. Sen, Nucl. Phys. B404 (1993) 109.

[109] J. Schwarz, CALT-68-1815.

[110] K. Kikkawa and M. Yamasaki, Phys. Lett. B149 (1984) 357; N. Sakai and I. Senda, Prog. Theor. Phys. 75 (1986) 692; T. Busher, Phys. Lett. B159 (1985) 127; V. Nair, A. Shapere, A. Strominger and F. Wilczek, Nucl. Phys. B322 (1989) 167; M. J. Duff, Nucl. Phys. B335 (1990) 610; A. A. Tseytlin and C. Vafa, Nucl. Phys. B372 (1992) 443; A. A. Tseytlin, Class. Quantum Grav. 9 (19920 979, A. Giveon, M. Porrati and E. Rabinovici, Phys. Rep. C (to appear).

[111] A. Font, L. Ibáñez, D. Lust and F. Quevedo, Phys. Lett. B249 (1990) 35.

[112] S. J. Rey, Phys. Rev. D43 (1991) 526.

[113] A. Sen, Phys. Lett. B303 (1993) 22.

[114] A. Sen, Mod. Phys. Lett. A8 (1993) 2023.

[115] J. H. Schwarz and A. Sen, Phys. Lett. B312 (1993) 105.

[116] M. J. Duff and J. Rahmfeld, CTP-TAMU-25/94, hepth/9406105.

[117] M. J. Duff and J. X. Lu, Nucl. Phys. B347 (1990) 394.

[118] D. J. Gross, R. D. Pisarski and L. G. Yaffe, Rev. Mod. Phys. 53 (1981) 43.

[119] B. R. Greene, A. Shapere, C. Vafa and S. T. Yau, Nucl. Phys. B337 (1990) 1.

[120] M. Cvetič, Phys. Rev. Lett. 71 (1993) 815.

[121] M. Cvetič, S. Griffies and H. H. Soleng, Phys. Rev. Lett. 71 (1993) 670; Phys. Rev. D48 (1993) 2613.

[122] H. S. La, Phys. Lett. B315 (1993) 51.

[123] M. J. Duff, NI-94-015, CTP-TAMU-22/94, hepth/9410046.

[124] M. J. Duff, NI-94-016, CTP-TAMU-48/94, hepth/9410210.

[125] D. Garfinkle, G. T. Horowitz and A. Strominger, Phys. Rev. D43 (1991) 3140.

[126] A. Shapere, S. Trivedi and F. Wilczek, Mod. Phys. Lett. A6 (1991) 2677. 
[127] R. Kallosh, A. Linde, T. Ortin, A. Peet and A. Van Proeyen, Phys. Rev. D46 (1992) 5278.

[128] R. Kallosh, Phys. Lett. B282 (1992) 80.

[129] R. Kallosh and T. Ortin, Phys. Rev. D48 (1993) 742.

[130] G. T. Horowitz, in Proceedings of Trieste '92, String theory and quantum gravity '92 p.55.

[131] A. Sen, Phys. Rev. Lett. 69 (1992) 1006.

[132] S. B. Giddings, J. Polchinski and A. Strominger, Phys. Rev. D48 (1993) 5784.

[133] D. J. Gross and M. J. Perry, Nucl. Phys. B226 (1983) 29.

[134] R. D. Sorkin, Phys. Rev. Lett. 51 (1983) 87.

[135] G. W. Gibbons and M. J. Perry, Nucl. Phys. B248 (1984) 629.

[136] C. F. E. Holzhey and F. Wilczek, Nucl. Phys. B360 (1992) 447.

[137] M. J. Duff and C. N. Pope, Nucl. Phys B255 (1985) 355.

[138] R. D'Auria, S. Ferrara and M. Villasante, Class. Quant. Grav. 11 (1994) 481.

[139] E. Cremmer, S. Ferrara, L. Girardello and A. Van Proeyen, Nucl. Phys. B212 (1983) 413.

[140] S. W. Hawking, Monthly Notices Roy. Astron. Soc. 152 (1971) 75; Abdus Salam in Quantum Gravity: an Oxford Symposium (Eds. Isham, Penrose and Sciama, O.U.P. 1975); G. 't Hooft, Nucl. Phys. B335 (1990) 138.

[141] M. Fabbrichesi, R. Jengo and K. ROland, Nucl. Phys. B402 (1993) 360.

[142] J. Ellis, N. E. Mavromatos and D. V. Nanopoulos, Phys. Lett. B278 (1992) 246.

[143] L. Susskind, RU-93-44, hepth/9309145; J. G. Russo and L. Susskind, UTTG-9-94, hepth/9405117.

[144] M. J. Duff, R. R. Khuri, R. Minasian and J. Rahmfeld, Nucl. Phys. B418 (1994) 195.

[145] G. W. Gibbons, in Supersymmetry, Supergravity and Related Topics, Eds. F. del Aguila, J. A. Azcarraga and L. E. Ibanez (World Scientific, 1985).

[146] P. Aichelburg and F. Embacher, Phys. Rev. D37 (1986) 3006.

[147] A. Hosoya, K. Ishikawa, Y. Ohkuwa and K. Yamagishi, Phys. Lett. B134 (1984) 44.

[148] G. W. Gibbons and D. L. Wiltshire, Ann. of Phys. 167 (1986) 201.

[149] G. C. Debney, R. P. Kerr and A. Schild, J. Math. Phys. 10 (1969) 1842.

[150] R. Geroch, J. Math. Phys. 13 (1972) 394.

[151] A. Dabholkar and J. A. Harvey, Phys. Rev. Lett. 63 (1989) 478.

[152] R. R. Khuri, Phys. Lett. B294 (1992) 331.

[153] N. S. Manton, Phys. Lett. B154 (1985) 397.

[154] M. F. Atiyah and N. J. Hitchin, Phys. Lett. A107 (1985) 21.

[155] M. F. Atiyah and N. J. Hitchin, The Geometry and Dynamics of Magnetic Monopoles, (Princeton University Press, 1988).

[156] R. R. Khuri, Phys. Lett. B307 (1993) 298. 
[157] G. W. Gibbons and S. W. Hawking, Phys. Rev. D15 (1977) 2752.

[158] G. W. Gibbons, S. W. Hawking and M. J. Perry, Nucl. Phys. B318 (1978) 141.

[159] D. Brill and G. T. Horowitz, Phys. Lett. B262 (1991) 437.

[160] R. R. Khuri, Nucl. Phys. B376 (1992) 350.

[161] A. G. Felce and T. M. Samols, Phys. Lett. B308 (1993) 30.

[162] J. Polchinski, Phys. Lett. B209 (1988) 252.

[163] R. R. Khuri, Phys. Rev. D48 (1993) 2823.

[164] I. Antoniadis, C. Bachas, J. Ellis and D. V. Nanopoulos, Phys. Lett. B211 (1988) 393.

[165] I. Antoniadis, C. Bachas, J. Ellis and D. V. Nanopoulos, Nucl. Phys. B328 (1989) 117.

[166] R. R. Metsaev and A. A. Tseytlin, Phys. Lett. B191 (1987) 354.

[167] R. R. Metsaev and A. A. Tseytlin, Nucl. Phys. B293 (1987) 385.

[168] D. Gepner and E. Witten, Nucl. Phys. B278 (1986) 493.

[169] C. Lovelace, Phys. Lett. B135 (1984) 75.

[170] P. Ginsparg, Lectures delivered at Les Houches summer session, June 28-August 5, 1988.

[171] E. Bergsheoff and M. de Roo, Nucl. Phys. B328 (1989) 439

[172] E. A. Bergshoeff and M. de Roo, Phys. Lett. B218 (1989) 210.

[173] R. W. Allen, I. Jack and D. R. T. Jones, Z. Phys. C41 (1988) 323.

[174] A. Sevrin, W. Troost and A. van Proeyen, Phys. Lett. B208 (1988) 447.

[175] K. Schoutens, Nucl. Phys. B295 [FS21] (1988) 634.

[176] C. Kounnas, in Proceedings of INFN Eloisatron Project, 26th Workshop: "From Superstrings to Supergravity", Erice, Italy, Dec. 5-12, 1992, Eds. M. Duff, S. Ferrara and R. Khuri, (World Scientific, 1994).

[177] G. T. Horowitz and A. A. Tseytlin, Phys. Rev. D50 (1994) 5204.

[178] A. A. Tseytlin, Imperial-TP-93-94-46, hepth/9407099.

[179] C. Klimcik and A. A. Tseytlin, Nucl. Phys. B424 (1994) 71.

[180] N. Seiberg and E. Witten, Nucl. Phys. B426 (1994) 19; RU-94-60, IASSNS-HEP 94/55, hepth/9408099.

[181] C. Vafa and E. Witten, HUTP-94-A017, hepth/9408074.

[182] J. Gauntlett and J. H. Harvey, EFI-94-30, hepth/9407111.

[183] P. H. Frampton and T. W. Kephart, IFP-708-UNC, VAND-TH-94-15.

[184] S. W. Hawking, G. T. Horowitz and S. F. Ross, NI-94-012, DAMTP/R 94-26, UCSBTH-94-25, gr-qc/9409013.

[185] G. W. Gibbons and R. Kallosh, NI-94003, hepth/9407118.

[186] G. T. Horowitz and A. A. Tseytlin, Imperial/TP/93-94/51, UCSBTH-94-24, hepth/9408040; Imperial/TP/93-94/54, UCSBTH-94-31, hepth/9409021. 
[187] M. Cvetič and D. Youm, UPR-623-T, hepth/9409119.

[188] E. Bergshoeff, R. Kallosh and T. Ortin, UG-8/94, SU-ITP-94-19, QMW-PH-94-13, hepth/9410230.

[189] S. Kalara and N. Nanopoulos, Phys. Lett. B267 (1992) 343.

[190] A. Sen, TIFR/TH/94-19, hepth/9408083.

[191] J. Maharana, Newton Institute preprint, October 1994.

[192] G. W. Gibbons, G. T. Horowitz and P. K. Townsend, R/94/28, UCSBTH-94-35, hepth/9410073.

[193] C. M. Hull and P. K. Townsend, QMW-94-30, R/94/33, hepth/9410167. 